\title{
ELECTRONIC PROPERTIES \\ OF SMALL METALLIC PARTICLES
}

\author{
J.A.A.J. PERENBOOM and P. WYDER \\ Physics Laboratory and Research Institute for Materials, University of Nijmegen, Nijmegen, \\ The Netherlands \\ and \\ F. MEIER
}

Laboratorium für Festkörperphysik, E.T.H., Zürich, Switzerland

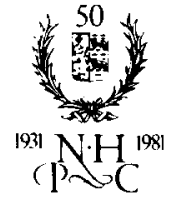

NORTHHOLLAND PUBLISHING COMPANY - AMSTERDAM 


\title{
ELECTRONIC PROPERTIES OF SMALL METALLIC PARTICLES
}

\author{
J.A.A.J. PERENBOOM and P. WYDER \\ Physics Laboratory and Research Institute for Materials, University of Nijmegen, Nijmegen, The Netherlands \\ and \\ F. MEIER \\ Laboratorium für Festkörperphysik, E.T.H., Zürich, Switzerland \\ Received April 1981
}

Contents:

1. Introduction 175

2. Phenomenological description of small particles $\quad 178$

2.1. Interaction with electromagnetic radiation 178

2.2. Mie's theory 178

2.3. The dielectric constant of a small metallic particle $\quad 184$

2.4. The effective dielectric constant of the embedding medium

3. Quantum size effects in small metallic particles

3.1. Microscopic description of electrons in small particles

3.2. Kubo's small particle

3.3. Level statistics

3.4. Spectroscopy of the small particle level structure

3.5. Experiments

4. Superconductivity in small particles

4.1. Characteristic lengths of superconductors
4.2. Magnetization of small superconducting particles 233

4.3. Microscopic theory of small superconductors 235

4.4. Fluctuations in small superconducting particles 251

5. Preparation of small particles $\quad 260$

5.1. Metal colloids 261

5.2. Granular metal films 264

5.3. Particles prepared by the gas-evaporation technique 269

5.4. Particles prepared by nucleation and growth in a matrix

$\begin{array}{ll}\text { 5.5. Impregnated porous materials } & 273 \\ & 275\end{array}$

Appendix I. Thermodynamic calculations for Kubo's small particle

Appendix II. Thermodynamic calculations for the particle with equal level spacing

References

Single orders for this issue

PHYSICS REPORTS (Review Section of Physics Letters) 78, No. 2 (1981) 173-292.

Copies of this issue may be obtained at the price given below. All orders should be sent directly to the Publisher. Orders must be accompanied by check.

Single issue price Dfl. 55.00 , postage included. 
Abstract:

Metallic particles with linear dimensions $d$ small compared with other characteristic lengths (like the wavelength of electromagnetic radiation. the de Broglie wavelength of the conduction electrons, the coherence length or the penetration depth in the superconducting state. etc.) show interesting effects which are usually unobservable in bulk metals. The electronic properties of these particles with diameters of a few nm can be analysed by considering the microcrystals not as "giant molecules" but as "small solids", i.e. by using the familiar methods of solid state physics with some properly defined boundary conditions. Due to the smallness of the particles, the customary quasi-continuous electronic excitation spectrum splits up into discrete energy levels with an average energy splitting $\delta$ of a few meV. If then the relevant energies (like the thermal energy $k T$, the Zeeman energy $\mu_{0} \mu_{\mathrm{B}} H$, the electrostatic energy $e d E$, the photon energy $h \omega$, the condensation energy for the superconducting state $\Delta$, etc.) are comparable with $\delta$, novel effects are to be expected, called "quantum size effects" (QSE). In an ensemble of small particles, it is expected that the discrete energy levels are statistically distributed; therefore, methods of level statistics can be employed to calculate the different electronic properties of small particles.

In this report, the more phenomenological aspects of the physics of small particles are discussed, where e.g. the interaction of the electromagnetic radiation with the particle is described by a dielectric constant, also characteristic for the bulk metal. The more microscopic quantum size effects in small particles are then analysed theoretically, mainly from the point of view of the statistics of discrete energy levels, and the existing experimental results are discussed. Superconductivity in small metallic particles is reviewed with emphasis on the critical fields in small particles, the magnetic field dependence of their microscopic properties (e.g. density of states), the problem of a lower size limit of a superconductor, and fluctuations in small superconductors. Finally, the most commonly used experimental methods to produce small particles are described.

\section{Introduction}

Between the well established and conventional domain of the atomic and molecular physics and that of the physics of condensed matter, there is an intermediate region dealing with the properties of small aggregates, clusters, or small particles, which are neither quite microscopic nor quite macroscopic. The study of systems in this intermediate region is rather important from a technological point of view for the understanding of problems related to catalysis, chemisorption, aerosols, powder metallurgy, ferrofluids, etc.; in addition, it is to be expected that the next level of miniaturization of devices will include systems belonging to this intermediate region. From a more fundamental point of view, the study of this state of matter, intermediate between that of a molecule and a solid, seems to be crucial and should be very interesting because it touches on some rather basic points of physical principles, usually concealed if one deals with infinitely large systems.

In this paper, we will limit ourselves exclusively to the electronic properties of small systems, i.e. the physical properties of small metallic particles due to the presence of electrons. Apart from a few but very important contributions [1-4] this subject seems to have attracted wider interest only during the last few years. Nevertheless, due to the many problems involved-experimentally it is very difficult to prepare well defined samples in this intermediate regime, and theoretically no adequate tools have been developed - the field of studies on the electronic properties of small metallic particles is still very much in its infancy. Some very relevant, more recent results can be found in the proceedings of the first and second conference on small particles [5,6], and the review papers by Baltes and Simanek [7], Hughes and Jain [8], Genzel [9], and Knight [10] give most valuable information.

We are interested in the change in the physical behaviour of a system if one puts together more and more identical atoms. Most crudely speaking, very obviously a collection of copper atoms and a piece of a copper wire will behave very much different, despite the fact that both consist of the same chemical element. The question is how to investigate this transition in the physical properties if one goes from a single atom to a bulk solid. Our point of view in this paper is to focus our attention on small metallic particles which still have a considerable number of electrons so that the usual methods of solid state physics remain applicable. In particular, the concept of a quasi-particle as an electronic excitation in a metal, is still supposed to be meaningful. We start from the usual ideas of solid state physics and add 
some modifications due to the smallness of the system as a sort of perturbation. In this spirit, a small metallic particle is then considered as a solid with dimensions $L$ smaller than some intrinsic length like the wavelength of light $\lambda$, the electron mean free path $l$, the London penetration depth of a superconductor $\lambda_{\mathrm{L}}$, the coherence length $\xi$ of the superconducting state, etc.

A rather interesting situation results from the quantum mechanical fact that a system consisting of many electrons in a finite volume has discrete energy levels. It is important to realize that a very clear distinction has to be made between the density of energy levels of the full thermodynamic many particle system, and the density of energy levels of one single quantum mechanical particle, both in a finite volume.

Generally speaking, for the full thermodynamic many particle system, the distances between the discrete energy levels of the whole many particle system decrease exponentially with increasing number $N$ of the particles. This is due to the well known fact from statistical mechanics [11], that in a closed system in thermal equilibrium the entropy $S$ may be expressed as a function of the total energy alone. The statistical weight $\Delta \Gamma$ (the number of quantum states of the full many particle system corresponding to the energy interval $\Delta E$ ) is then by definition given by:

$$
\Delta \Gamma=\mathrm{e}^{s(E)} .
$$

Dividing $\Delta E$ by $\Delta \Gamma$, we obtain the mean separation between neighbouring levels in this interval near the energy $E$. Denoting this distance by $\Delta(E)$, we get:

$$
\Delta(E)=\Delta E \mathrm{e}^{-S(E)}
$$

Therefore, the entropy function $S(E)$ determines the density of levels in the energy spectrum of a macroscopic system. Since the entropy is additive, the mean spacings between the levels of a macroscopic body consisting of the full many particle system decrease exponentially with increasing number $N$ of particles in it. It is clear that this analysis is closely related to the fact that surface effects can be neglected compared with bulk effects if the number of particles involved is sufficiently large and therefore it is possible to define i.e. the entropy per particle $s=S / N$. In the explicit calculation of $s$ the conceptually different energy density of the one particle system comes into play, which can be a function of the dimensions of the system as well. In the rest of this paper, we shall be concerned with this one-particle density of states only.

If we take a one-electron model Hamiltonian for the conduction electrons to represent the "quasi-particles" in the small metallic particle, the eigenvalues and eigenfunctions are determined by the appropriate boundary conditions. Usually, the level systematics of particles in a large volume is obtained by considering the system in a large cube with periodic boundary conditions. In a naïve free-electron model, the energy levels are given by $E_{n}=\hbar^{2} \pi^{2} n^{2} /\left(2 m^{*} L^{2}\right)$, where $L$ is the linear dimension of the system, $m^{*}$ the effective mass of the electrons, and $n^{2}=n_{x}^{2}+n_{y}^{2}+n_{z}^{2}$, with integer values for $n_{\alpha}$. The spacing between two neighbouring levels at the Fermi energy $\varepsilon_{\mathrm{F}}$ is then given by $\Delta \varepsilon=\hbar^{2} \pi^{2} /\left(2 m^{*} L^{2}\right)$. This situation, where the final levels correspond to values of the momentum quantized in intervals $\Delta p_{\alpha} \simeq \hbar / L$, is perfectly adequate for the description of a macroscopic system, and takes into account the uncertainty relation $\Delta x_{\alpha} \Delta p_{\alpha} \geq \hbar$, which will broaden the levels accordingly. In a naive, but incorrect picture one could argue that the levels should be broader than $\Delta E \geq \hbar \Delta t$, and estimate the mean life time of the Bloch states from the size limited mean free path: $\Delta E \geq \hbar v_{\mathrm{F}} / L$; the enormous broadening of the single particle energy levels found in this case is rather an indication of the 
breakdown of the naïve quasi-one-dimensional Bloch model. Yet, it makes some sense to consider $L / v_{\mathrm{F}}$ as a kind of lifetime: In his discussion of the width of the electron spin resonance peak measured in small metallic particles Kawabata [12] showed that this classical estimate corresponds to the strength of the transition matrix elements in a quantum mechanical calculation.

If we consider the electron levels of sufficiently minute metallic particles, imperfections in the shape of such particles will remove the artificial degeneracy of the system due to the periodic boundary conditions. Then the average distance between two levels at the Fermi energy is $\delta=\Delta \varepsilon /\left(2 \pi n_{\mathrm{F}} / 8\right)$ (where $n_{\mathrm{F}}=L p_{\mathrm{F}} /(\pi h)$, and $p_{\mathrm{F}}$ the Fermi momentum), and is of course just twice the inverse of the density of states $\rho\left(\varepsilon_{\mathrm{F}}\right)$ at the Fermi level. Also then, for sufficiently small particles, the level spacing may be larger than the broadening of the single electron energy levels. In a particle of volume $V=L^{3}$, containing $N$ electrons, we therefore have for spin degenerate levels:

$$
\delta=2 / \rho\left(\varepsilon_{\mathrm{F}}\right)=2 \pi^{2} \hbar^{3} /\left(V p_{\mathrm{F}} m^{*}\right)=\frac{4}{3} \varepsilon_{\mathrm{F}} / N
$$

For a small gold particle with diameter $d=10 \mathrm{~nm}$, and using the coefficient $\gamma$ in the linear law of the electronic specific heat as measured in the bulk material, eq. (1.3) gives $\delta=0.10 \mathrm{meV}$. In general it is seen from eq. (1.3) that when $N \simeq 10^{4}, \ldots 10^{5}$ the average spacing is $\delta / k \simeq 1, \ldots 0.1 \mathrm{~K}$; this is quite large even for particle dimensions which are sufficiently large so that still the macroscopic characteristics of the metal can be used. It is obvious, and was realized many years ago [2-4], that interesting effects should occur if the average value for an ensemble of small particles of the level spacing near the Fermi energy $\delta$ becomes bigger than the thermal energy $k T$, the Zeeman energy $\mu_{0} \mu_{\mathrm{B}} H$ ( $H$ is the applied magnetic field), the electrostic energy edE ( $E$ is the applied electric field), the energy $\hbar \omega$ of radiation, etc. (See fig. 1.1.) In a most remarkable paper [3], it was Kubo who reactivated interest in and triggered more recent theoretical and experimental work on these problems.

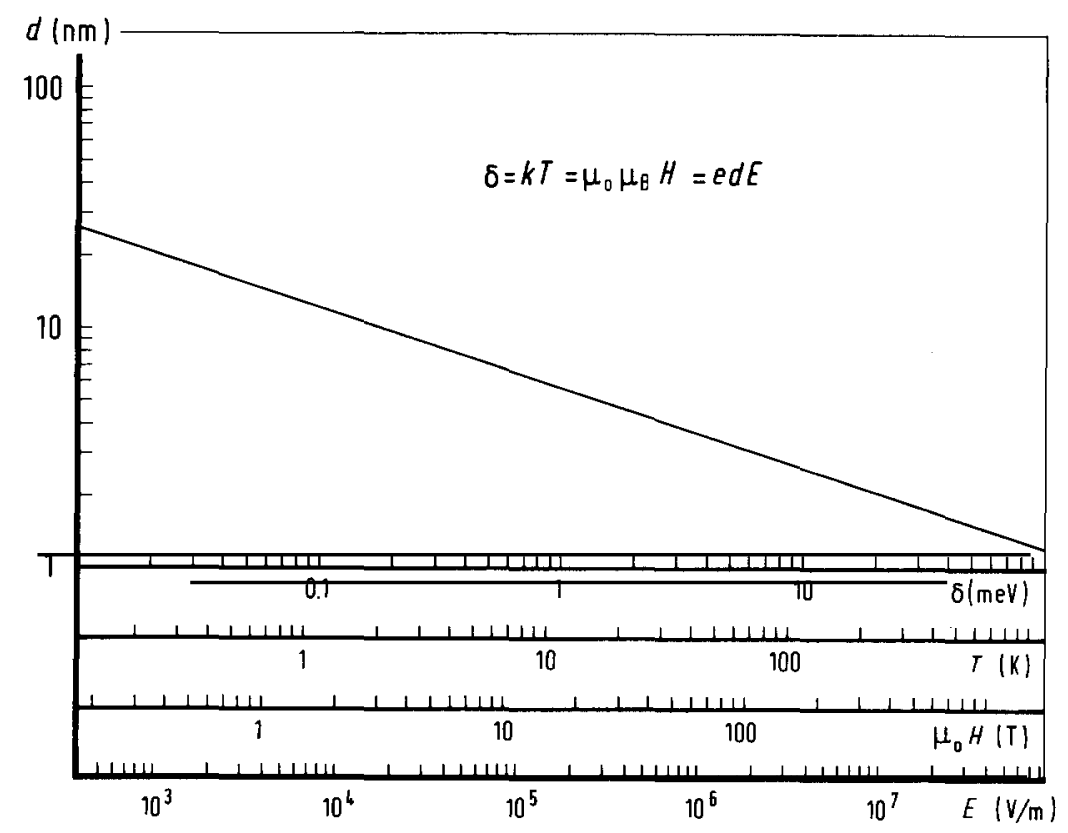

Fig. 1.1. Average level spacing $\delta=4 \varepsilon_{\mathrm{F}} / 3 N$ as a function of the particle diameter $d$, measured in terms of some other relevant energies. The parameters have been calculated using a typical "standard metal", with a value of $N / V=6.0 \times 10^{28}$ electrons $/ \mathrm{m}^{3}$ for the electron density. 
It should be emphasized that the finiteness of the dimensions of the particles brings about two distinct effects on the electronic states in a particle. The first is the discreteness of the energy levels as given by eq. (1.3), and the second is the effect due to the existence of surfaces as discussed in connection with the full thermodynamic manybody problem. These surface effects will certainly be important under the circumstances discussed here, but we will be mainly concerned with the first effect, namely that of the discreteness of the one-particle energy levels due to the smallness of the system. In principle, a small particle should be considered as a giant molecule composed of many thousands of atoms, but here we will consider a small particle as a small solid.

\section{Phenomenological description of small particles}

\subsection{Interaction with electromagnetic radiation}

Small metallic particles or colloids often show beautiful colours, usually different from the colours of the bulk metal. An explanation for this phenomenon was given early in this century in terms of the classical electromagnetic theory [1,13]. A particle, exposed to electromagnetic radiation, will reduce the intensity of the incident beam by scattering and absorption. This problem can be analysed with Maxwell's equations. The discussion of this problem is relatively simple, because the dimensions of the submicroscopic particles of interest here are smaller than the wavelength of the incoming electromagnetic wave (if we consider only nonionizing radiation). The applied field may then be assumed to be homogeneous over the volume of the particle. For the case of a spherical particle a full solution of the boundary problem was worked out by Mie [1]. In this phenomenological treatment the complex dielectric constants of the metal and of the surrounding medium are the only material properties which enter the calculation. Therefore, Mie's theory can be applied to metals as well as to ionic crystals and semiconductors [9]. The extension of Mie's theory to ellipsoidally shaped particles is quite easy, but also for different particle geometries solutions may be found [14].

Normally one is interested in the properties of a collection of more or less identical particles, reasonably well separated from each other, and embedded in a medium with well defined dielectric constant: an appreciable fraction of the volume of the embedding medium will be occupied by small particles. The theory of J.C. Maxwell Garnett [13] gives a prescription how to calculate the effective dielectric constant of a medium containing a small volume fraction of particles. When the particles occupy a large fraction of the volume, as in some composite materials, this theory fails. The evaluation of the effective dielectric constant is then possible only in so far as it is possible to characterize the material explicitly. This problem will be discussed in some detail in section 2.4. In section 2.2 we will consider the solution given by Mie and section 2.3 will discuss size effects on the dielectric constant of the metal particles.

\subsection{Mie's theory}

We will now turn to the discussion of Mie's calculation of the interaction of small spherical particles with electromagnetic radiation. The calculation is straightforward, but rather tedious, and was repeatedly elaborated by many illustrious workers [15-17]. Avoiding detailed calculations we will present a brief outline of the procedure to calculate the attenuation of an unpolarized monochromatic light beam after it has passed through a medium containing spherical particles. The volume concen- 
tration of particles is assumed to be so low that the particles act as independent scattering centers and that multiple scattering does not take place. Then the extinction of the beam is given by:

$$
I(z)=I_{0} \mathrm{e}^{-\gamma z}
$$

where $I(z)$ is the intensity of the incoming beam after a distance $z$. Under these conditions, the extinction coefficient $\gamma$ is a simple product of the number of scattering centers per unit volume $N / V$ and the extinction cross section of one particle $C_{\text {ext }}$. The extinction cross section is the sum of the scattering cross section $C_{\text {sca }}$ and the absorption cross section $C_{\text {abs }}$ :

$$
\gamma=\frac{N}{V} C_{\mathrm{ext}}=\frac{N}{V}\left(C_{\mathrm{sca}}+C_{\mathrm{abs}}\right)
$$

This extinction cross section can now be calculated with Mie's theory, starting from Maxwell's equations. We consider the case of a monochromatic, linearly polarized plane wave of electric and magnetic field amplitudes $E$ and $B$ incident onto the particle $P$ (see fig. 2.1). As a consequence of Maxwell's equations, $E$ and $B$ are not independent: $B=(n / c) E$, where $n=\sqrt{\varepsilon \mu}$ is the refractive index of the medium. (Throughout this paper $\varepsilon$ and $\mu$ will denote the relative permittivity and permeability respectively.) The incoming field expressed in polar coordinates is given by:

$$
\begin{aligned}
& E_{r}=\sin \theta \cos \phi \mathrm{e}^{\mathrm{i} k r \cos \theta} \mathrm{e}^{-\mathrm{i} \omega t} \\
& E_{\theta}=\cos \theta \cos \phi \mathrm{e}^{\mathrm{i} k r \cos \theta} \mathrm{e}^{-\mathrm{i} \omega t} \\
& E_{\phi}=-\sin \phi \mathrm{e}^{\mathrm{i} k r \cos \theta} \mathrm{e}^{-\mathrm{i} \omega t}
\end{aligned}
$$

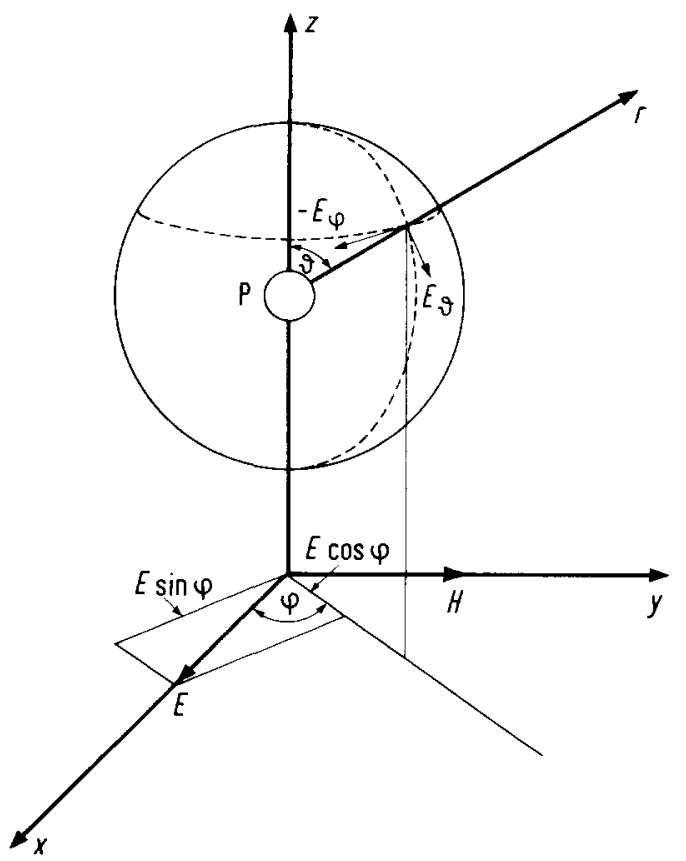

Fig. 2.1. The incoming electromagnetic wave, directed along the $+z$-axis, and the outgoing spherical wave, which is scattered by the particle at position $P$. 


$$
\begin{aligned}
& B_{r}=(n / c) \sin \theta \sin \phi \mathrm{e}^{\mathrm{i} k r \cos \theta} \mathrm{e}^{-\mathrm{i} \omega t} \\
& B_{\theta}=(n / c) \cos \theta \sin \phi \mathrm{e}^{\mathrm{i} k r \cos \theta} \mathrm{e}^{-\mathrm{i} \omega t} \\
& B_{\phi}=(n / c) \cos \phi \mathrm{e}^{\mathrm{i} k r \cos \theta} \mathrm{e}^{-\mathrm{i} \omega t}
\end{aligned}
$$

It was noted by Debye [15] that this problem can most easily be solved when we realize that the vectors $\boldsymbol{E}$ and $\boldsymbol{B}$ can be derived from a scalar field $V$ which satisfies the same wave equation as the cartesian components of $\boldsymbol{E}$ and $\boldsymbol{B}$, i.e.: $\Delta V+k^{2} V=0$. One obtains all possible solutions by considering two different functions $V_{1}$ and $V_{2}$; the first will correspond to the case where the radial part of $\boldsymbol{B}$ is vanishing and electrical waves are generated, the latter will represent the magnetic waves with vanishing $E_{r}$. The complete solution is the superposition of the fields derived from the two potentials. For the electrical waves the components of $\boldsymbol{E}$ can be found as derivatives of the function $(\partial / \partial r) r V_{1}$ as will be shown below, and the contribution of $r V_{1}$ to the magnetic field is obtained subsequently by using Maxwell's equations. For the magnetic waves, in a similar way the components of $\boldsymbol{B}$ are obtained from direct differentiation of $(\partial / \partial r) r V_{2}$, and the contribution to the electric field is found again from the corresponding Maxwell equation:

$$
\begin{aligned}
& E_{r}=\frac{\partial^{2}}{\partial r^{2}} r V_{1}+k^{2} r V_{1} \\
& E_{\theta}=\frac{1}{r} \frac{\partial^{2}}{\partial r \partial \theta} r V_{1}+\frac{i c k}{n r \sin \theta} \frac{\partial}{\partial \phi} r V_{2} \\
& E_{\phi}=\frac{1}{r \sin \theta} \frac{\partial^{2}}{\partial r \partial \phi} r V_{1}-\frac{i c k}{n r} \frac{\partial}{\partial \theta} r V_{2} \\
& B_{r}=\frac{\partial^{2}}{\partial r^{2}} r V_{2}+k^{2} r V_{2} \\
& B_{\theta}=-\frac{i n k}{c r \sin \theta} \frac{\partial}{\partial \phi} r V_{1}+\frac{1}{r} \frac{\partial^{2}}{\partial r \partial \theta} r V_{2} \\
& B_{\phi}=\frac{i n k}{c r} \frac{\partial}{\partial \theta} r V_{1}+\frac{1}{r \sin \theta} \frac{\partial^{2}}{\partial r \partial \phi} r V_{2} .
\end{aligned}
$$

The expressions for $E_{r}$ and $B_{r}$ can be used to get $r V_{1}$ and $r V_{2}$ respectively. The solution can be written as a series expansion in spherical harmonics:

$$
\begin{aligned}
& r V_{1}^{(1)}=\frac{1}{k_{1}^{2}} \mathrm{e}^{-\mathrm{i} \omega t} \cos \phi \sum_{l=1}^{\infty} \mathrm{i}^{l-1} \frac{2 l+1}{l(l+1)} \alpha_{l} \psi_{l}\left(k_{1} r\right) P_{l}^{(1)}(\cos \theta) \\
& r V_{2}^{(1)}=\frac{n}{c k_{1}^{2}} \mathrm{e}^{-\mathrm{i} \omega t} \sin \phi \sum_{l=1}^{\infty} \mathrm{i}^{l-1} \frac{2 l+1}{l(l+1)} \beta_{l} \psi_{l}\left(k_{1} r\right) P_{l}^{(1)}(\cos \theta)
\end{aligned}
$$

Here $n$ is the (complex) index of refraction and $k=n \omega / c$ the wavevector in the medium; $\psi_{l}(z)[15]$ is a Ricatti-Bessel function of the type $(\pi z / 2)^{1 / 2} J_{l+1 / 2}(z)$ where $J_{l+1 / 2}(z)$ is a half integral Bessel function, and $P_{l}^{(1)}(\cos \theta)$ is an associated Legendre polynomial. For $\alpha_{l}=\beta_{l}=1$ the eqs. (2.5a) and (2.5b) will 
generate the incoming wave given by eq. (2.3). For the field inside the particle, an ansatz of the form given in eq. (2.5) is appropriate. For the scattered wave outside the particle a decomposition must be made with functions $\eta_{l}(k r)$ [15] instead of $\psi_{l}(k r) . \eta_{l}(z)$ is a Ricatti-Bessel function of the type $(\pi z / 2)^{1 / 2} H_{l+1 / 2}^{(1)}(z)$ where $H_{l+1 / 2}^{(1)}(z)$ is a half integral Hankel function of the first kind. The asymptotic form of $\eta_{l}(k r)$ for $k r \gg 1$ is $\eta_{l}(k r)=(-\mathrm{i})^{l+1} \mathrm{e}^{\mathrm{i} k r}$, representing an outgoing wave. The ansatz for the scattered wave is therefore:

$$
\begin{aligned}
& r V_{1}^{(2)}=\frac{1}{k_{2}^{2}} \mathrm{e}^{-\mathrm{i} \omega t} \cos \phi \sum_{l=1}^{\infty} \mathrm{i}^{l-1} \frac{2 l+1}{l(l+1)} a_{l} \eta_{l}\left(k_{2} r\right) P_{l}^{(1)}(\cos \theta) \\
& r V_{2}^{(2)}=\frac{n_{0}}{c k_{2}^{2}} \mathrm{e}^{-\mathrm{i} \omega t} \sin \phi \sum_{l=1}^{\infty} \mathrm{i}^{l-1} \frac{2 l+1}{l(l+1)} b_{l} \eta_{l}\left(k_{2} r\right) P_{l}^{(1)}(\cos \theta)
\end{aligned}
$$

Here and in the following, the index (1), (2), and (i) refer to the particle, the embedding medium, and the incoming wave respectively. The coefficients $\alpha_{l}, \beta_{l}, a_{l}$ and $b_{l}$ can be determined from the boundary conditions at the surface of the particle:

$$
\begin{aligned}
& E_{\text {tang. }}^{(1)}=E_{\text {tang. }}^{(\mathrm{i})}+E_{\text {tang. }}^{(2)} . \\
& B_{\text {tang. }}^{(1)}=B_{\text {tang. }}^{(i)}+B_{\text {tang. }}^{(2)} .
\end{aligned}
$$

The continuity of $E_{\theta}$ and $E_{\phi}$ implies the continuity of the functions $(\partial / \partial r) r V_{1}$ and $r V_{2}$ at $r=\frac{1}{2} d$, where $d$ is the particle diameter. Similarly, the continuity of $k^{2} r V_{1}$ and $(\partial / \partial r) r V_{2}$ follows from the continuity of both $B_{\theta}$ and $B_{\phi}$ at the particle surface. The coefficients $a_{l}$ and $b_{l}$ for the scattered wave found from the boundary conditions are:

$$
\begin{aligned}
& a_{l}=-\frac{n \psi_{l}(n x) \psi_{l}^{\prime}\left(n_{0} x\right)-n_{0} \psi_{l}^{\prime}(n x) \psi_{l}\left(n_{0} x\right)}{n \psi_{l}(n x) \eta_{l}^{\prime}\left(n_{0} x\right)-n_{0} \psi_{l}^{\prime}(n x) \eta_{l}\left(n_{0} x\right)} \\
& b_{l}=-\frac{n_{0} \psi_{l}(n x) \psi_{l}^{\prime}\left(n_{0} x\right)-n \psi_{l}^{\prime}(n x) \psi_{l}\left(n_{0} x\right)}{n_{0} \psi_{l}(n x) \eta_{l}\left(n_{0} x\right)-n \psi_{l}^{\prime}(n x) \eta_{l}\left(n_{0} x\right)} .
\end{aligned}
$$

These expressions contain the Mie-parameter $x=\frac{1}{2} k_{0} d$, with the vacuum wavevector $k_{0}=2 \pi / \lambda_{0}$, the index of refraction of the embedding medium $n_{0}=\sqrt{\varepsilon_{\mathrm{m}}}$, and the (complex) index of refraction of the particle $n=\sqrt{\varepsilon}$. Far away from the particle, $r \gg \lambda$, we can substitute the asymptotic form of the function $\eta_{l}\left(k_{2} r\right)$. We define functions $S_{1}(\theta)$ and $S_{2}(\theta)$ :

$$
\begin{aligned}
& S_{1}(\theta)=\sum_{l=1}^{\infty} \frac{2 l+1}{l(l+1)}\left\{a_{l} \frac{P_{l}^{(1)}(\cos \theta)}{\sin \theta}+b_{l} \frac{\mathrm{d} P_{l}^{(1)}(\cos \theta)}{\mathrm{d} \theta}\right\} \\
& S_{2}(\theta)=\sum_{l=1}^{\infty} \frac{2 l+1}{l(l+1)}\left\{a_{l} \frac{\mathrm{d} P_{l}^{(1)}(\cos \theta)}{\mathrm{d} \theta}+b_{l} \frac{P_{l}^{(1)}(\cos \theta)}{\sin \theta}\right\} .
\end{aligned}
$$

Then the components of the electric and magnetic fields can be expressed conveniently as: 


$$
\begin{aligned}
& \left(\begin{array}{c}
E_{\phi} \\
-B_{\theta}
\end{array}\right)=\frac{\mathrm{i} \exp \left\{i k_{2} r-\mathrm{i} \omega t\right\}}{k_{2} r} \sin \phi S_{1}(\theta)\left(\begin{array}{l}
E \\
B
\end{array}\right) \\
& \left(\begin{array}{l}
E_{\theta} \\
B_{\phi}
\end{array}\right)=-\frac{\left.i \exp { }_{1} k_{2} r-\mathrm{i} \omega t\right\}}{k_{2} r} \cos \phi S_{1}(\theta)\left(\begin{array}{l}
E \\
B
\end{array}\right) \\
& E_{r}=B_{r}=0 .
\end{aligned}
$$

Far away from the particle, the scattered wave has the form of an outgoing transverse spherical wave. The forward scattered beam $(\theta=0)$ determines the extinction cross section. Because of the fact that

$$
\left.\frac{1}{\sin \theta} P_{l}^{(1)}(\cos \theta)\right|_{\theta=0}=\left.\frac{\mathrm{d}}{\mathrm{d} \theta} P_{l}^{(1)}(\cos \theta)\right|_{\theta=0}=\frac{1}{2} l(l+1)
$$

the functions $S_{1}(0)$ and $S_{2}(0)$ are equal and the extinction cross section is independent of the state of polarization of the incident radiation. The extinction and scattering cross sections can now be calculated from the amplitude function $S(0)$ by:

$$
\begin{aligned}
& C_{\mathrm{ext}}=-\frac{\pi d^{2}}{\left(n_{0} x\right)^{2}} \operatorname{Re}\{S(0)\}=-\frac{2 \pi}{n_{0}^{2} k_{0}^{2}} \sum_{l=1}^{\infty}(2 l+1) \operatorname{Re}\left\{a_{l}+b_{l}\right\} \\
& C_{\mathrm{sca}}=\frac{2 \pi}{n_{0}^{2} k_{0}^{2}} \sum_{l=1}^{\infty}(2 l+1)\left\{\left|a_{l}\right|^{2}+\left|b_{l}\right|^{2}\right\}
\end{aligned}
$$

We can proceed to an evaluation of the cross sections by a series expansion in powers of $x$ of the functions entering eqs. (2.8a) and (2.8b). The lowest order term in $C_{\text {ext }}$ is proportional to $x^{3}$ and stems from $a_{1}$, the third term in the expansion of $a_{1}$ is proportional to $x^{6}$ and is related with the first term in the power series for $C_{\text {sca. }}$. For very small particles, only the first order electric wave will contribute, and the extinction will be mainly due to absorption. The series expansion of the coefficients leads to the following result:

$$
\begin{aligned}
& a_{1}=\frac{2}{3} \mathrm{i} n_{0}^{3} \frac{n^{2}-n_{0}^{2}}{n^{2}+2 n_{0}^{2}} x^{3}+\frac{2}{5} \mathrm{i} n_{0}^{5} \frac{\left(n^{2}-n_{0}^{2}\right)\left(n^{2}-2 n_{0}^{2}\right)}{\left(n^{2}+2 n_{0}^{2}\right)^{2}} x^{5}-\frac{4}{9} n_{0}^{6}\left(\frac{n^{2}-n_{0}^{2}}{n^{2}+2 n_{0}^{2}}\right)^{2} x^{6} \\
& b_{1}=\frac{1}{45} \mathrm{i} n_{0}^{3}\left(n^{2}-n_{0}^{2}\right) x^{5} \\
& a_{2}=\frac{1}{15} \mathrm{i} n_{0}^{5} \frac{n^{2}-n_{0}^{2}}{2 n^{2}+3 n_{0}^{2}} x^{5} .
\end{aligned}
$$

For small enough particles, the extinction can be calculated by substitution of the lowest order term in $a_{1}$ only. With $x=\frac{1}{2} k_{0} d, n^{2}=\varepsilon=\varepsilon_{1}+\mathrm{i} \varepsilon_{2}$, and $n_{0}^{2}=\varepsilon_{\mathrm{m}}$ the extinction coefficient $\gamma$ is calculated to be:

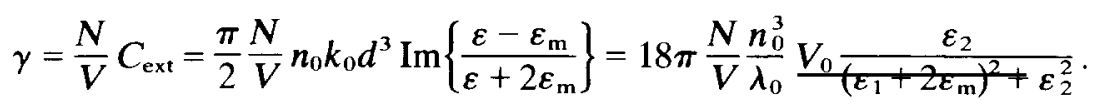

$\gamma$ is proportional to the volume of the particle $V_{0}$ and the number of particles per unit volume. The 
intensity of the scattered radiation will be proportional to $V_{0}^{2}$, as can be inferred from eq. (2.11b). The range of validity of eq. (2.13) has to be checked in each case by comparison with the full Mie expression eq. (2.11).

The scattered radiation field calculated from the Mie theory can be considered as generated by a superposition of oscillating electric and magnetic multipoles. The coefficients $a_{l}$ and $b_{l}$ determine the magnitude of the corresponding $l$ th multipole moments. In the optical frequency range and for very small particles ( $d \leq 10^{-1} \lambda$, where $\lambda$ is the vacuum wavelength of the electromagnetic radiation) only the electric dipole contribution needs to be taken into account.

The result of the Mie theory given in eq. (2.13) can be considered as being due to an electric plasma oscillation induced in the metallic particle. In this context, it is very illustrative to calculate the electric dipole moment $\boldsymbol{p}$ induced by an applied electric field $\boldsymbol{E}_{0}$ in a particle with dielectric constant $\varepsilon$ embedded in a medium with dielectric constant $\varepsilon_{\mathrm{m}}$. For the calculation of the strength of the electric field the charges at the interface of the particle and the medium have to be taken into account. In the particularly simple geometry of spherical particles, the depolarizing field is homogeneous over the volume of the particle and the field inside the particle is proportional to the applied field. The field outside the particle is the superposition of the applied field and the field of the electric dipole moment induced in the particle. When the field $\boldsymbol{E}_{0}$ is directed along the $z$-axis, the electric field can be calculated as the gradient of the scalar field $\Phi$ given by:

$$
\begin{aligned}
& \Phi^{(1)}=-\alpha E_{0} r \cos \theta \\
& \Phi^{(2)}=-E_{0} r \cos \theta+\frac{p \cos \theta}{4 \pi \varepsilon_{0} r} .
\end{aligned}
$$

The proportionality factors $p$ and $\alpha$ can be calculated from the usual boundary conditions:

$$
\begin{aligned}
& \boldsymbol{E}_{\text {tang. }}^{(1)}=\boldsymbol{E}_{\text {tang. }}^{(2)} \\
& \varepsilon \boldsymbol{E}_{\text {perp. }}^{(1)}=\varepsilon_{\mathrm{m}} \boldsymbol{E}_{\text {perp. }}^{(2)} .
\end{aligned}
$$

We obtain for the field inside the metallic particle:

$$
\boldsymbol{E}^{(1)}=\frac{3 \varepsilon_{\mathrm{m}}}{\varepsilon+2 \varepsilon_{\mathrm{m}}} \boldsymbol{E}_{0}
$$

and for the induced electric dipole moment:

$$
p=\frac{\pi}{6} d^{3} \frac{\varepsilon-\varepsilon_{\mathrm{m}}}{\varepsilon+2 \varepsilon_{\mathrm{m}}} 3 \varepsilon_{0} E_{0}
$$

The plasma oscillation of the metallic sphere is given by the frequency $\omega_{R}$ for which $p$ becomes infinite, i.e. when:

$$
\varepsilon\left(\omega_{\mathrm{R}}\right)+2 \varepsilon_{\mathrm{m}}=0
$$

This electric dipole plasma resonance corresponds to the lowest order contribution to the scattering in 
the Mie theory given in eq. (2.13), and leads to the well known beautiful colours of submicroscopic dispersions of metals.

\subsection{The dielectric constant of a small metallic particle}

The Mie theory for the scattering and absorption of electromagnetic radiation by a small metallic particle, as discussed in the previous section, leads to a plasma resonance. This plasma oscillation is usually found in the visible or near ultraviolet and has been studied by many workers using transmission of radiation [18-35], energy loss experiments in the interaction between fast electrons and the small particles [36,37], by direct observation of the intensity of the scattered light [38], and this local plasmon mode is possibly observed also in the emitted light from particles contained in tunnel junctions when the particles are excited by inelastic tunneling electrons [39]. In the interpretation of the optical properties of small particles, it was soon realized that the limited mean free path of the electrons is the most important factor leading to modifications of the optical constant of finely dispersed metals [18-20]: The mean free path for diffuse scattering off the surface of a spherical particle is $\frac{1}{2} d$, and will be smaller than the bulk mean free path for sufficiently small crystallites. The corresponding reduction of the lifetime of the electron states leads for small $d$ to a broadening of the resonance proportional to the particle diameter. In fig. 2.2 the plasma resonance is shown of small silver particles as measured by Doremus [22] in one of his pioneering studies. The absorption is calculated from Mie's theory, using experimental values for the bulk dielectric constant of silver, and the imaginary part was corrected for the reduced mean free path. The overall correspondence between theory and experiment is satisfactory. Extensive studies of the optical properties of small silver and gold particles by Kreibig et al. [25-27] have confirmed this result.

It is generally accepted that a good approximation to the dielectric constant in small particles is easily obtained from the bulk optical constants: The contribution due to interband transitions $\varepsilon_{b}(\omega)$ is mostly believed to remain unchanged, but the Drude-like free electron part should be modified using a size limited scattering time:

$$
1 / \tau=1 / \tau_{0}+2 v_{\mathrm{F}} / d
$$

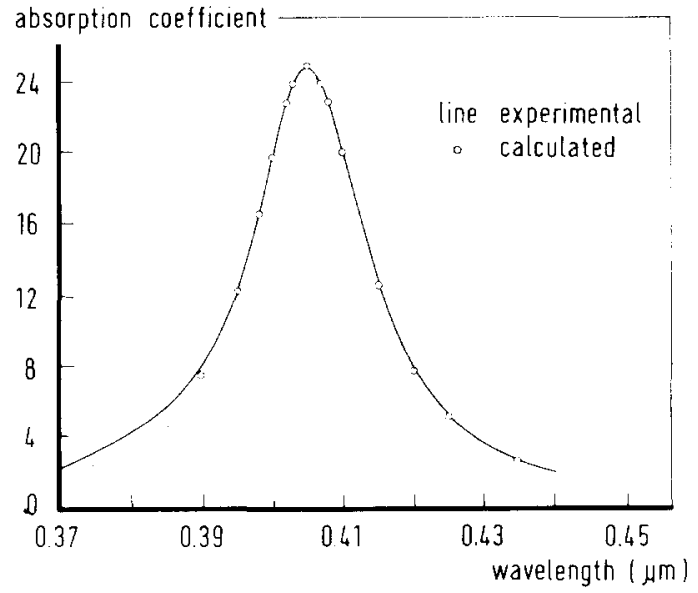

Fig. 2.2. The optical plasma resonance absorption line for small silver particles with an average diameter of about $10 \mathrm{~nm}$. The values indicated by the circles, calculated using a Drude-like dielectric constant with a mean free path of the electrons limited by the size of the particle, are in good agreement with the experimental curve. (From Doremus [22].) 
$\tau_{0}$ is the scattering time in the bulk material, and $v_{\mathrm{F}}$ denotes the Fermi velocity. The contribution of the surface scattering becomes appreciable in the size range below $100 \mathrm{~nm}$. The resulting dielectric constant can then be written as:

$$
\varepsilon(\omega)=\varepsilon_{\mathrm{b}}(\omega)-\frac{\omega_{\mathrm{p}}^{2}}{\omega(\omega+\mathrm{i} / \tau)}=\varepsilon_{\mathrm{b}}(\omega)-\frac{\omega_{\mathrm{p}}^{2} / \omega^{2}}{1+1 / \omega^{2} \tau^{2}}+\mathrm{i} \frac{\omega_{\mathrm{p}}^{2} / \omega^{2}}{\omega \tau\left(1+1 / \omega^{2} \tau^{2}\right)}
$$

where $\omega_{\mathrm{p}}$ denotes the plasma frequency of the bulk material.

For very small particles a new effect comes into play: Due to size quantization, the conduction band will break up into discrete levels with an average separation large compared to thermal energies $[2,3]$. This effect is called Quantum Size Effect (QSE). The Drude expression of eq. (2.20) will then no longer be valid. Several attempts have been made to derive a dielectric function relevant for this situation quantum mechanically [26,40-44]. Kawabata and Kubo [40] have argued that the classical interpretation of mean free path limitation by scattering at the surface is not correct: The surface of particles in this size range does not really scatter the electrons, it rather manifests itself as a boundary condition for electron states bound to the finite volume of the particle. The dissipative part $\varepsilon_{2}$ of the dielectric constant represents dipole transitions between these eigenstates, and has been calculated on the basis of general principles [40]. As discussed in Ruppin's paper [44], the expressions found for $\varepsilon_{2}$ by different authors agree with the result originally obtained by Kawabata and Kubo:

$$
\varepsilon_{2}^{\mathrm{OM}}(\omega)=\frac{32}{\varepsilon_{0} \pi^{4}} \frac{e^{2} \varepsilon_{\mathrm{F}}^{2}}{\hbar^{3} \omega^{3}} \frac{1}{d} g_{\mathrm{s}}\left(\frac{\hbar \omega}{\varepsilon_{\mathrm{F}}}\right)=\frac{12}{\pi^{2}} \frac{\omega_{\mathrm{p}}^{2}}{\omega^{3}} \frac{v_{\mathrm{F}}}{d} g_{\mathrm{s}}\left(\frac{\hbar \omega}{\varepsilon_{\mathrm{F}}}\right)
$$

$g_{\mathrm{s}}$ is a smoothly varying function and has a value of the order of 1 . This result should be compared with the phenomenological result as given in eq. (2.20) in the limit for small particles, when $1 / \tau \simeq 2 v_{\mathrm{F}} / d$ :

$$
\varepsilon_{2}^{\text {Drude }}(\omega) \simeq 2 \omega_{\mathrm{p}}^{2} v_{\mathrm{F}} / \omega^{3} d
$$

The observed line width of the plasma resonance of small silver particles in fig. 2.3 [26] is consistent

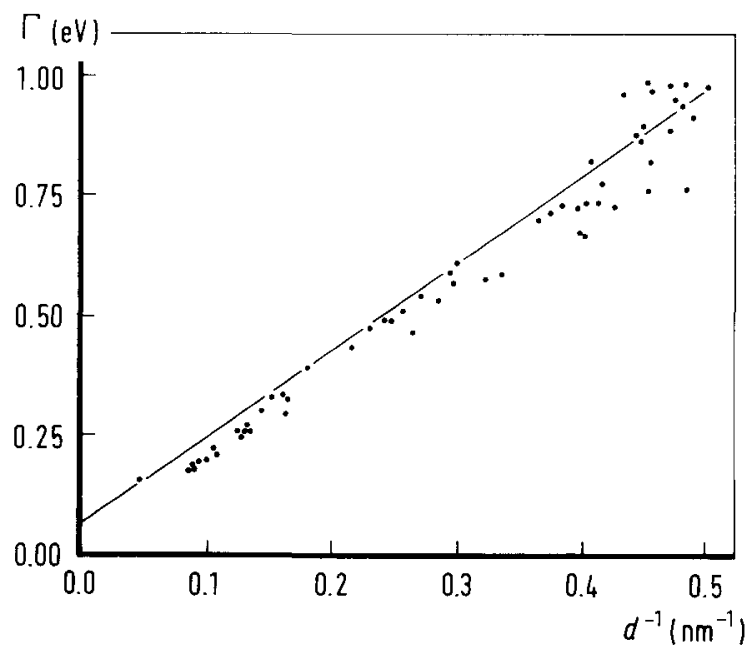

Fig. 2.3. Measured line width $\Gamma$ of the optical plasma resonance peak in small silver particles as a function of the inverse particle diameter $d^{-1}$. (From Genzel, Martin and Kreibig [26].) 
with this $d^{-1}$-dependence, as given in eq. (2.21) or eq. (2.22). However, the quantum mechanical calculations predict an effective mean free path for a spherical particle of approximately twice the value of the classical estimate leading to a line width which is considerably smaller than the one observed. It seems that the classical interpretation of the effect compares better with experiment than the quantum mechanical calculations which take into account the quantum size effects. It should be noted however that anomalously short scattering times can result from lattice defects in small metallic particles [45]. In addition, the size distribution in samples containing larger particles tends to broaden the resonance peak.

The situation is even more controversial concerning the position of the peak of the plasma resonance. The classical Mie theory takes only into account Maxwell's equations, and therefore the resulting resonances are purely geometrical in origin. If higher order terms in the Mie parameter $x$ contribute to the extinction given by eq. (2.11), the shape of the resonance will change and the position $\lambda_{\max }$ of the peak will shift. For small particles, higher order terms can be neglected and the position of the peak will be fixed, unless some size dependence of the dielectric constant comes into play. This is illustrated by model calculations for small sodium particles of Smithard and Tran [30], shown in fig. 2.4. It can be seen that the peak hardly shifts for particles with diameter below approximately $10 \mathrm{~nm}$. For even smaller particles the classical concept of the limitation of the mean free path will lead to a shift of the peak position towards longer wavelengths.

Let us consider again the Drude dielectric function as given in eq. (2.20). The frequency $\omega_{\mathrm{R}}$ of the electric dipole plasma resonance is then found from the condition $\varepsilon_{1}\left(\omega_{\mathrm{R}}\right)=-2 \varepsilon_{\mathrm{m}}$ (eq. (2.18)). Using eq. (2.20) we obtain:

$$
\frac{\omega_{\mathrm{p}}^{2} / \omega_{\mathrm{R}}^{2}}{1+\left(1 / \omega_{\mathrm{R}} \tau_{0}+2 v_{\mathrm{F}} / \omega_{\mathrm{R}} d\right)^{2}}=\varepsilon_{\mathrm{b}}\left(\omega_{\mathrm{R}}\right)+2 \varepsilon_{\mathrm{m}}
$$

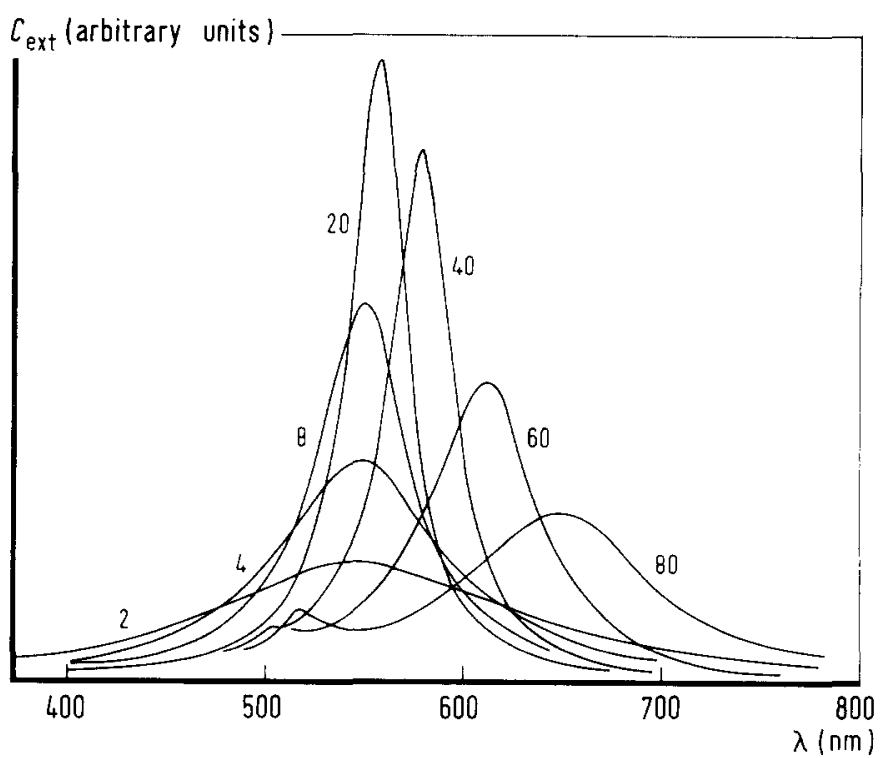

Fig. 2.4. Absorption spectrum for small sodium particles, calculated using Mie's theory. For the dielectric constant the classical Drude-expression was used with a size limited mean free path. The numbers indicate the diameter of the particles in nm. (From Smithard and Tran [30].) 
The resonance frequency is large compared to the inverse scattering time $\left(\omega_{\mathrm{R}} \tau_{0} \gg 1\right)$, but for particles with a diameter approaching $1 \mathrm{~nm} 2 v_{\mathrm{F}} / \omega_{\mathrm{R}} d$ will be comparable to 1 . For particles substantially larger than $1 \mathrm{~nm}$ eq. (2.23) reduces to the size independent value of:

$$
\omega_{\mathrm{R}}^{2}=\frac{\omega_{\mathrm{p}}^{2}}{\varepsilon_{\mathrm{b}}\left(\omega_{\mathrm{R}}\right)+2 \varepsilon_{\mathrm{m}}}
$$

Eq. (2.23) will lead to a shift of $\omega_{R}$ to longer wavelengths. Smithard and coworkers have indeed observed such a shift in small silver [28,29] and sodium [30,31] particles. Around the resonance frequency $\omega_{R}$, the expression for the extinction cross section shows a strong frequency dependence (as $\left.\omega^{4}\right)$ which in general results in a shift of the actual peak towards frequencies higher than $\omega_{R}$. For sodium particles, the position of the peak will remain approximately constant for particles down to $1 \mathrm{~nm}$, in agreement with the model calculations shown in fig. 2.4 .

The results of a model calculation by Jain and Arora [32] for small silver particles is given in fig. 2.5. Here, a dielectric function has been used which has been derived quantum mechanically from the results of Kawabata and Kubo [40] based on the QSE. The behaviour is strikingly different from the curves shown in fig. 2.4 based on the classical results: For the smallest particle diameters, they show a decrease of the wavelength at which maximum absorption occurs. Genzel, Martin and Kreibig have derived a quantum mechanical dielectric function, as well; they considered the simple model of a particle represented by electrons moving in a cubic potential well with infinitely high sides [26, 46]. The experimentally observed line width (fig. 2.3) and position of the peak of the plasma resonance (fig. 2.6)

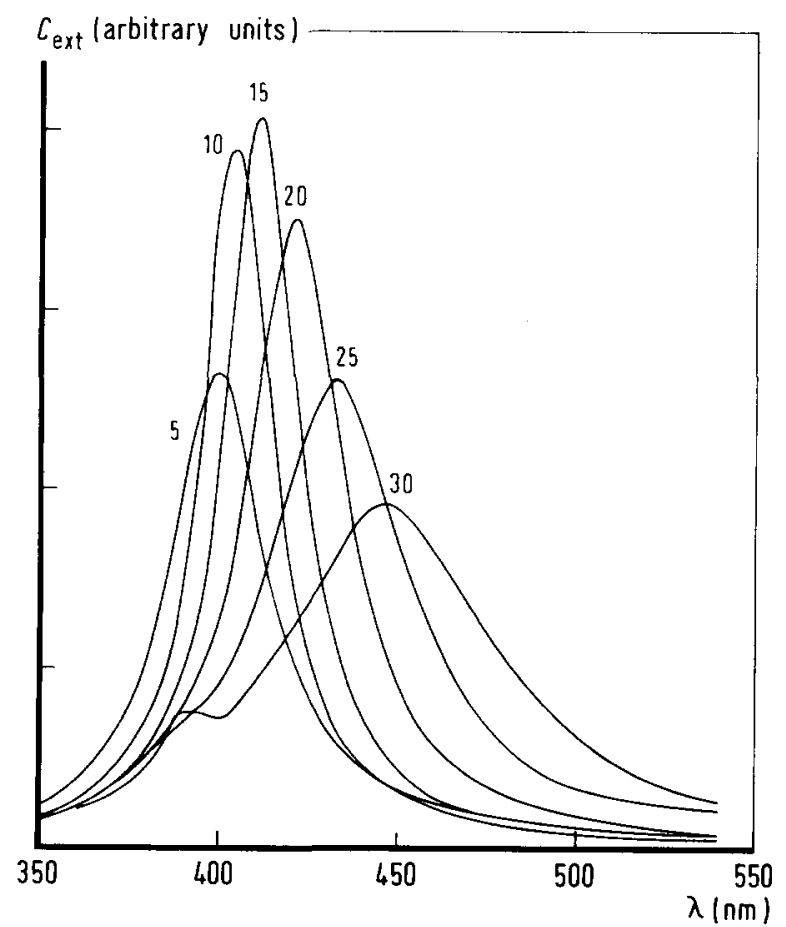

Fig. 2.5. Absorption spectrum for small silver particles, calculated using Mie's theory. The dielectric constant was calculated using the quantum mechanical theory of Kawabata and Kubo [40] which takes quantum size effects into account. The numbers indicate the diameter of the particles in nm. (From Jain and Arora [32].) 


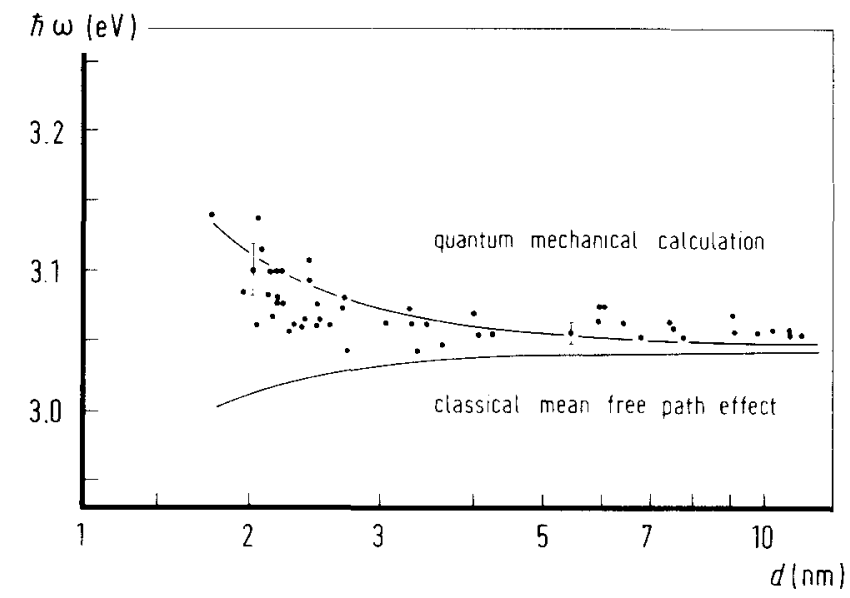

Fig. 2.6. Position of the optical plasma resonance peak as a function of the particle diameter for small silver particles. The data seem to compare better with the quantum mechanical calculations than with calculations using a classical size limited mean free path. (From Genzel, Martin and Kreibig [26].)

are in agreement with this model. This shift to higher frequencies of the position of the plasma resonance with decreasing particle size was also found in other quantum mechanical calculations [32, 47] based on the original work of Kawabata and Kubo [40]. In an attempt to give a simplified analytical solution, they argue that the dielectric function can be given in a Drude-like form as

$$
\varepsilon(\omega)=\varepsilon_{\mathrm{b}}(\omega)-\frac{\omega_{\mathrm{p}}^{2}}{\left(\omega^{2}-\Omega^{2}\right)^{2}+\omega^{2} / \tau^{2}}\left\{\left(\omega^{2}-\Omega^{2}\right)-i \omega / \tau\right\}
$$

The new constant $\Omega$ can be found from the expression for the dielectric constant for zero frequency, using the results of their model calculation:

$$
\varepsilon_{1}(0)=\varepsilon_{\text {bulk }}(0)+0.028 \frac{e^{2} m n^{1 / 3}}{\varepsilon_{0} \hbar^{2}} L^{2}=\varepsilon_{\text {bulk }}(0)+0.27 \frac{\omega_{\mathrm{p}}^{2}}{v_{\mathrm{F}}^{2}} L^{2}
$$

When we express eq. (2.26) in terms of an effective scattering length $L_{\mathrm{eff}}=(2 / \pi) L$, we obtain

$$
\Omega^{2}=1.52 v_{\mathrm{F}}^{2} / L_{\mathrm{eff}}^{2}
$$

Therefore, $\Omega \ll \omega_{\mathrm{p}}$ even for particles with a diameter of $1 \mathrm{~nm}$. From this result, a strong frequency dependence in the infrared is expected for the dielectric function [46]. The result of eq. (2.26) has been derived by Cini and Ascarelli [42], as well and is in agreement with the high static susceptibility of small metallic particles predicted by Gor'kov and Eliashberg [4] many years ago. As before, eq. (2.25), together with the resonance condition eq. (2.18), can be used to calculate the position of the quantum mechanical plasma resonance $\omega_{\mathrm{QM}}$ of a small particle. $\omega_{\mathrm{QM}}$ will only deviate slightly from $\omega_{\mathrm{R}}$ as calculated from eq. (2.24):

$$
\omega_{\mathrm{OM}}^{2}-\Omega^{2}+\frac{\omega_{\mathrm{OM}}^{2}}{\omega_{\mathrm{QM}}^{2}+\Omega^{2}} \frac{1}{\tau^{2}}=\omega_{\mathrm{R}}^{2} .
$$


Using $1 / \tau=1 / \tau_{0}+v_{\mathrm{F}} / L_{\mathrm{eff}}$ the position of the peak is found to be:

$$
\omega_{\mathrm{QM}}^{2} \simeq \omega_{\mathrm{R}}^{2}+\Omega^{2}-1 / \tau^{2} \simeq \omega_{\mathrm{R}}^{2}+0.52 v_{\mathrm{F}}^{2} / L_{\mathrm{eff}}^{2} .
$$

As before, the frequency at which maximum absorption is found, will be shifted to higher frequencies even more. This shift of the plasma resonance to higher frequencies for the data represented in fig. 2.6 seems to support the quantum mechanically derived dielectric function. For silver and sodium particles a shift of the plasma peak to lower frequencies was found [28-31]; several attempts have been made to reconcile the seemingly contradictory experimental results: The influence of surface diffuseness [48, 49], and of the inclusion of a dielectric nucleus $[50,51]$ have been considered to explain a shift towards longer wavelengths. In addition, the extension of the classical Mie theory to include longitudinal polarization waves in the small particles revealed a possible secondary absorption structure just above the plasma frequency $\omega_{\mathrm{p}}[52]$.

\subsection{The effective dielectric constant of the embedding medium}

The presence of polarizable particles will give an extra contribution to the effective dielectric constant of the embedding medium. The field inside a polarizable inclusion is depressed below the applied field as a result of the depolarization field of the charges accumulated at the interface between. the particle and the medium; the average field outside the polarizable inclusions exceeds the space average field. Therefore a higher fieldstrength will be effective in polarizing an additional particle.

In eq. (2.17) we have calculated the net electric dipole moment induced in a particle embedded in the medium. The extra polarization per unit volume $\Delta \boldsymbol{P}$ resulting from the induced dipole moments is proportional to the volume fraction of metal $f$ and can be written as:

$$
\Delta \boldsymbol{P}=3 f \frac{\varepsilon-\varepsilon_{\mathrm{m}}}{\varepsilon+2 \varepsilon_{\mathrm{m}}} \varepsilon_{0} \boldsymbol{E}_{\mathrm{loc}}
$$

where $\boldsymbol{E}_{\mathrm{loc}}$ is the field acting on the particle. We have to take into account the field at the position of an additional particle resulting from all the induced dipole moments. This is relatively simple if we consider the usual spherical region around this particle. The contributions from all the dipole moments inside the Lorentz sphere just cancel; the contributions of all the dipole moments outside the sphere reduce to the depolarization field of the charges at the interface of the medium and the hypothetical Lorentz sphere without a particle

$$
\boldsymbol{E}_{\mathrm{loc}}=\varepsilon_{\mathrm{m}} \boldsymbol{E}_{0}+\frac{1}{3} \Delta \boldsymbol{P} / \varepsilon_{0}=\frac{1}{3}\left(\varepsilon_{\mathrm{eff}}+2 \varepsilon_{\mathrm{m}}\right) \boldsymbol{E}_{0}
$$

where we have introduced $\varepsilon_{\text {eff }}$ as the effective dielectric constant of the medium together with the particles. Using again $\Delta \boldsymbol{P}=\left(\varepsilon_{\text {eff }}-\varepsilon_{\mathrm{m}}\right) \varepsilon_{0} \boldsymbol{E}_{0}$, substitution of eq. (2.31) in eq. (2.30) leads to the following result:

$$
\frac{\varepsilon_{\mathrm{eff}}-\varepsilon_{\mathrm{m}}}{\varepsilon_{\mathrm{eff}}+2 \varepsilon_{\mathrm{m}}}=f \frac{\varepsilon-\varepsilon_{\mathrm{m}}}{\varepsilon+2 \varepsilon_{\mathrm{m}}}
$$

This result reminds one of the familiar Clausius-Mossotti approximation and has been originally derived 
by J.C. Maxwell Garnett [13]. It is often written in an alternative form:

$$
\varepsilon^{\mathrm{MG}}=\varepsilon_{\mathrm{eff}}=\varepsilon_{\mathrm{m}} \frac{1+2 f\left(\varepsilon-\varepsilon_{\mathrm{m}}\right) /\left(\varepsilon+2 \varepsilon_{\mathrm{m}}\right)}{1-f\left(\varepsilon-\varepsilon_{\mathrm{m}}\right) /\left(\varepsilon+2 \varepsilon_{\mathrm{m}}\right)}
$$

The Maxwell Garnett theory has been very successful in explaining the optical properties of submicroscopic dispersions of metals. Part of this success is due to the intimate relationship with the Mie theory [1]: Both theories are based on the solution of the same type of boundary condition problem. The Maxwell Garnett theory is believed to be accurate only for sufficiently low metal volume concentration and for particles smaller than the wavelength of the radiation inside the medium. In fact, even stronger requirements must hold [33,53]: The Maxwell Garnett theory is valid only when all higher order terms in the Mie theory can be neglected and eq. (2.13) holds.

In recent years there has been an increasing interest for composite materials where the volume fractions of metal and dielectric are comparable. An extension of the Maxwell Garnett theory to higher metal volume fraction or a careful discussion of its limits of accuracy is therefore important. In the reviews by Landauer [54] and Hale [55] a good analysis of this field is given. The dielectric constant of a composite does not only depend on the dielectric constants of the (two) phases and the respective volume fractions, it depends also on the more microscopic geometry of the composite material. The need for detailed structural information sets a limit to the possibility to predict the dielectric constant of inhomogeneous materials. In fact only bounds can be given for the value of the effective dielectric constant. Hashin and Shtrikman [56] have shown that the Maxwell Garnett result of eq. (2.33) gives a rigorous lower bound for $\varepsilon_{\text {eff }}$ as a function of metal volume fraction; eq. (2.33) is the exact solution for the dielectric constant of a material where the space within the composite is completely filled by spheres of varying diameter each surrounded by a dielectric shell with a thickness appropriate to the metal volume fraction of the composite. Inversely, the composite sphere assemblage, where the roles of the two composites are reversed, gives a rigorous upper bound for $\varepsilon_{\mathrm{eff}}$. When some knowledge of the phase geometry is available, more stringent bounds can be set on the range of values of $\varepsilon_{\text {eff }}$.

A serious objection to the Maxwell Garnett result of eq. (2.33) is the asymmetry in the treatment of the two different materials. This has been taken into account by Bruggeman's effective medium theory [57], where both components are treated in a fully equivalent way: Both dielectric and metal particles are considered as inclusions in a medium with dielectric constant $\varepsilon^{\mathrm{EMT}}$. The effective medium result is obtained when $\varepsilon^{\mathrm{EMT}}$ is chosen in such a way that fluctuations due to the dipole fields of the inclusions average out to zero:

$$
f \frac{\varepsilon-\varepsilon^{\mathrm{EMT}}}{\varepsilon+2 \varepsilon^{\mathrm{EMT}}}+(1-f) \frac{\varepsilon_{\mathrm{m}}-\varepsilon^{\mathrm{EMT}}}{\varepsilon_{\mathrm{m}}+2 \varepsilon^{\mathrm{EMT}}}=0 .
$$

This is exactly the original result of Bruggeman for spherical inclusions and can be formulated alternatively as:

$$
\varepsilon^{\mathrm{EMT}}=\varepsilon_{\mathrm{m}} \frac{1-f+f\left(\varepsilon-\varepsilon^{\mathrm{EMT}}\right) /\left(\varepsilon+2 \varepsilon^{\mathrm{EMT}}\right)}{1-f-2 f\left(\varepsilon-\varepsilon^{\mathrm{EMT}}\right) /\left(\varepsilon+2 \varepsilon^{\mathrm{EMT}}\right)}
$$

For small $f$ Bruggeman's effective medium theory reduces to the original Maxwell Garnett result. For a higher volume fraction of metal the effective medium theory should be more accurate. It seems 
however, that this approach has not given better agreement with the experimental results than the Maxwell Garnett theory [58-60].

The extension of the theory to non-spherical particles has been discussed by Polder and van Santen [61] and, more recently, by Galeener [62]. Ellipsoidal shape of the inclusions can be introduced simply by substituting the appropriate depolarization factor $L$ in the denominator at the right hand side of eq. (2.32) instead of the factor of $\frac{1}{3}$ relevant to spherical inclusions. In the case of aligned ellipsoids as discussed by Galeener one single factor $L$ is needed. For the case of randomly oriented ellipsoids an averaging has to be carried out over the possible orientations of the ellipsoids with respect to the electric field. Galeener's results have been criticized by Cohen et al. [63]: For extreme values of the depolarization factor, correct values of the effective dielectric constant are obtained only, when an ellipsoidal cavity congruent with the particle is considered, instead of a Lorentz sphere; the same depolarization factor $L$ will then appear on both sides in eq. (2.32).

In the case discussed by Galeener, the polarizability per unit volume of the voids is independent of the void size and their shape is reflected only through the factor $L$. In small metallic inclusions the dielectric constant will be size dependent, as discussed in the preceding section. Therefore, a summation over the size distribution of the metallic particles should be included. Granquist and coworkers have used the result of Polder and van Santen for a selfconsistent formulation of the Maxwell Garnett theory $[33,34]$. They have explicitly expressed the size dependence using a $d$-dependent volume fraction $f(d)$, and they obtained for the case of randomly oriented ellipsoids:

$$
\bar{\varepsilon}=\varepsilon_{\mathrm{m}} \frac{1+\frac{2}{3} \sum_{d} f(d) \frac{1}{3} \sum_{i}(\varepsilon(\underline{d})-\bar{\varepsilon}) /\left(\bar{\varepsilon}+L_{i}(\varepsilon(d)-\bar{\varepsilon})\right)}{1-\frac{1}{3} \sum_{d} f(d) \frac{1}{3} \sum_{i}(\varepsilon(d)-\bar{\varepsilon}) /\left(\bar{\varepsilon}+L_{i}(\varepsilon(d)-\bar{\varepsilon})\right)}
$$

In discontinuous films, one axis of the ellipsoidal islands is normally found to be oriented perpendicular to the substrate. Then the averaging is over two possible depolarization factors $L_{i}$ only [34].

There is considerable interest in the properties of particulate materials, because the absorption resonance in the visible, found in this type of inhomogeneous material, can be used to make effective coatings for solar energy conversion [64-66]. It was noted that the small particles often touch and are arranged in chains of spheres, even when the filling factor is low. When the particles are evenly spread and wide apart, it may be expected that the effective field of all the other particles acting on an additional particle is well described using the Lorentz sphere approach. When the individual particles touch and form complex chains or clusters, local field effects arise which have not yet been taken into account. Granqvist and Hunderi [33] have tried to incorporate local field effects by the introduction of effective depolarization factors. This approach was based on the work of Clippe, Evrard and Lucas [67], who calculated the resonance frequency for a number of geometrical configurations of identical touching spheres. This introduction of effective depolarization factors has rather drastic effects [33, 34]. Moreover, the effective dielectric constant will increase with increasing metal volume fraction. Therefore, the Maxwell Garnett theory predicts a shift to longer wavelengths of the plasma resonance with increasing $f$. Similarly, a shift to longer wavelengths is expected when one of the depolarization factors is reduced below $\frac{1}{3}$, because the resonance condition is now modified to:

$$
L \varepsilon+(L-1) \bar{\varepsilon}=0
$$

Granqvist and Hunderi were able to give a very accurate description of the measured transmittance of gas evaporated gold particles [33] using a distribution of effective depolarization factors. Also in the 
case of discontinuous films, the high absorption and the shift of the absorption peak found experimentally could be reconciled with the simple Maxwell Garnett theory when local field effects were taken into account $[34,68]$. The extension of the original Maxwell Garnett theory to incorporate local field effects is still part of active research $[69,70]$.

As far as most experiments are concerned, it is obvious that the Maxwell Garnett theory is very successful. One of the most serious shortcomings of this theory is the fact that no percolation threshold is predicted. In this respect, the effective medium theory is an improvement [71]. On the other hand, Bruggeman's theory does not take into account the all important microstructure of the coated spheres, so that the surface plasma resonance predicted by Mie's theory becomes washed out for increasing metal content, in contradiction with experimental findings. However, very recently Ping Sheng [72] has demonstrated that a rigorous mixing of the two possible microgeometries of metal particles surrounded by dielectric and metal coated dielectric particles leads to both a percolation threshold and a plasma resonance.

\section{Quantum size effects in small metallic particles}

\subsection{Microscopic description of electrons in small particles}

\subsubsection{Electronic quantum size effects}

Quantum size effects (QSE) in small metallic particles have been discussed for the first time in a paper by Fröhlich [2], published in 1937. Fröhlich applies the Sommerfeld-Bloch model of free electrons in a bulk metal directly to a particle of very small dimensions, and the modifications to the usual, bulk value of the electronic specific heat are discussed. Although nowadays his approach seems too unrealistic to give meaningful results, the basic idea of electronic QSE appears clearly.

It is shown that metallic matter in form of sufficiently small grains behaves qualitatively different from the bulk metal. Relying upon Fröhlich's original paper we turn now to the first and most important question: What does 'small' mean in the context of QSE, or: How small must a particle be that its intrinsic properties become different from those of a bulk solid?

\subsubsection{What is a small particle?}

When the number of atoms contained in a grain of solid matter is steadily reduced, it is plausible that in course of this process a stage is realized, where the particle does not behave like a smaller copy of the corresponding bulk solid anymore. A lower limit for the critical size is a particle consisting of one single atom. However, for most physical properties one expects not a sharp transition from atomic to bulk solid behaviour; rather, one may expect a gradual change as a function of the number of atoms and ambient conditions, as temperature, pressure and electromagnetic fields.

There are two obvious approaches to a quantitative estimate of this transition. The first one is to start from a single atom and to build up particles by adding atom after atom, and to calculate the electronic states, vibration modes and electromagnetic properties from molecular orbital theory. This procedure needs enormous computational efforts and generally the cluster of atoms must not contain more than about 100 atoms to keep the work within reasonable proportions [73-79]. Besides, information obtained from such calculations frequently has only the value of examples: It does not give physical insight based on some general and simple principles.

Therefore we shall be mostly concerned with another approach, at least when dealing with electronic 
properties: the framework of bulk solid state theory will be used to a large extent to describe the particle properties, but modifications are added where the physical model makes them necessary. Clearly such a procedure cannot furnish correct results for a particle consisting of 5 or 10 atoms. But it should give useful information when the small particle is produced by gradually reducing the size of a macroscopic solid and when the conditions are such that it still contains thousands of atoms at the onset of the transition. This small particle approach from the bulk side is exactly what Fröhlich did.

Following Fröhlich [2], we consider the electronic specific heat of a small metallic particle. The specific heat is a measure for the energy which has to be supplied to the electron gas in order to increase its temperature by $\Delta T$. When the electronic energy levels are denoted by $\varepsilon_{n}$ and their degeneracy by $g_{n}$, the energy difference $\Delta U$ at a temperature $T$ with respect to the ground state energy $(T=0) U_{0}$ is:

$$
\Delta U=U-U_{0}=\sum_{n=0}^{\infty} g_{n} \varepsilon_{n} f_{n}-\sum_{m=0}^{m_{0}} g_{m} \varepsilon_{m}
$$

where $\varepsilon_{m_{0}}$ is the Fermi energy $\varepsilon_{\mathrm{F}}$ and $f_{n}$ the Fermi distribution function

$$
f_{n}=\frac{1}{\exp \left\{\left(\varepsilon_{n}-\varepsilon_{\mathrm{F}}\right) / k T\right\}+1} .
$$

In order to derive the specific heat $C_{\mathrm{e}}=\partial(\Delta U) / \partial T$, a scheme must be devised to find the levels $\varepsilon_{n}$ and their degeneracy $g_{n}$. When this has been accomplished, the problem is well defined, whatever the nature of the system under consideration may be.

The energy levels $\varepsilon_{n}$ can be calculated simply for a free electron gas enclosed in a cube of lateral length $L$. Then the single electron energies are given by:

$$
\varepsilon_{n}=\frac{\hbar^{2} k_{n}^{2}}{2 m}=\frac{\hbar^{2}}{2 m}\left(\frac{\pi}{L}\right)^{2}\left(n_{1}^{2}+n_{2}^{2}+n_{3}^{2}\right), \quad n_{i}=0,1,2, \ldots
$$

$\boldsymbol{k}_{n}=(\pi / L)\left(n_{1}, n_{2}, n_{3}\right)$ is the wavevector of a standing electron wave. The endpoints of the $\boldsymbol{k}_{n}$-vectors form a simple cubic lattice in the positive octant of the $k$-space.

If the system contains a large number of electrons (as it is supposed to do) $n^{2}=n_{1}^{2}+n_{2}^{2}+n_{3}^{2}$ is a large number for states near the Fermi energy. As almost any large natural number can be represented as the sum of three squares in many ways, the energy differences $\Delta \varepsilon$ between successive levels at the Fermi energy is given by:

$$
\Delta \varepsilon=\frac{\hbar^{2}}{2 m}\left(\frac{\pi}{L}\right)^{2}
$$

As the density of states of a free electron gas is a monotonically increasing function of energy, and as the energy difference $\Delta \varepsilon$ between successive levels is independent of energy (see eq. (3.4)), to each $\varepsilon_{n}$ is assigned a certain degree of degeneracy such that the total number of states is fixed. If the free electron energy level density is denoted by $\rho(\varepsilon)-$ a continuous function of energy! - the number of states between $\varepsilon$ and $\varepsilon+\Delta \varepsilon$ is $\rho(\varepsilon) \Delta \varepsilon$, provided that $\Delta \varepsilon \ll \varepsilon$. As there are no states between $\varepsilon$ and $\varepsilon+\Delta \varepsilon$ in Fröhlich's model of discrete energy levels, the state $\varepsilon$ itself must have the degeneracy $\rho(\varepsilon) \Delta \varepsilon$ in order to conserve the correct total number of states. 
Now we proceed to determine $g_{m}$ in the free electron approximation. The volume of a shell between $\varepsilon$ and $\varepsilon+\Delta \varepsilon$ in the positive octant of the $k$-space is given by:

$$
\Omega=\frac{\pi}{4} \frac{(2 m)^{3 / 2}}{\hbar^{3}} \sqrt{ } \bar{\varepsilon} \Delta \varepsilon
$$

The density of $k$-points is $(\pi / L)^{-3}$, and therefore the density of states, taking spin degeneracy into account, is given by $\rho(\varepsilon) \Delta \varepsilon=2 \Omega /(\pi / L)^{3}$ :

$$
\rho(\varepsilon)=L^{3} \frac{1}{2 \pi^{2}} \frac{(2 m)^{3 / 2}}{\hbar^{3}} \sqrt{\varepsilon}
$$

This is the continuous level distribution of the free electron model which has the property that $N(\varepsilon)=\int_{0}^{\varepsilon} \rho\left(\varepsilon^{\prime}\right) \mathrm{d} \varepsilon^{\prime}$, where $N(\varepsilon)$ is the number of states up to energy $\varepsilon$. When instead of the continuous level distribution of eq. (3.6), a discrete distribution is introduced, where the difference between successive levels is constant and independent of energy (see eq. (3.4)), the normalization $N(\varepsilon)=$ $\int_{0}^{\varepsilon} \rho\left(\varepsilon^{\prime}\right) \mathrm{d} \varepsilon^{\prime}$ has to be replaced by

$$
N\left(\varepsilon_{n}\right)=\sum_{m=0}^{n} g_{m}
$$

where $g_{m}$ denotes the degree of degeneracy of the level $\varepsilon_{m}$. Necessarily, one has

$$
g_{m}=\rho\left(\varepsilon_{m}\right) \Delta \varepsilon
$$

As an estimate for the order of magnitude involved, we calculate $\Delta \varepsilon$ and $g_{m}$ for a cube of lateral length L. Assuming $\varepsilon_{\mathrm{F}}=5 \mathrm{eV}$, one gets

$$
\begin{array}{llll}
L=1 \mathrm{~cm}: & \Delta \varepsilon=4 \times 10^{-15} \mathrm{eV}, & 4 \times 10^{-11} \mathrm{~K} ; & g_{m}=6 \times 10^{7} \\
L=10 \mathrm{~nm}: & \Delta \varepsilon=4 \times 10^{-3} \mathrm{eV}, \quad 40 \mathrm{~K} ; & g_{m}=60 .
\end{array}
$$

The position of $\varepsilon_{\mathrm{F}}$ between its neighbouring upper and lower levels $\varepsilon_{1}$ and $\varepsilon_{-1}$ is determined by the usual normalization procedure. We consider here the simple case, that $\varepsilon_{F}$ lies halfway between $\varepsilon_{1}$ and $\varepsilon_{-1}$. With the degeneracy of the level $\varepsilon_{n}$ given by $\rho\left(\varepsilon_{n}\right) \Delta \varepsilon, \Delta U$ of eq. (3.1) becomes

$$
\Delta U=\sum_{n=1,2 . .} \rho\left(\varepsilon_{n}\right) \Delta \varepsilon \varepsilon_{n} f_{n}-\sum_{n=1,2 .} \rho\left(\varepsilon_{-n}\right) \Delta \varepsilon \varepsilon_{-n}\left(1-f_{-n}\right)
$$

As $f_{n}$ falls from the value 1 to 0 over the energy width $k T$, and as $k T \ll \varepsilon_{\mathrm{F}}, \rho\left(\varepsilon_{ \pm n}\right)$ may be put equal to $\rho\left(\varepsilon_{\mathrm{F}}\right)$ and considered as constant. The first term of eq. (3.1) is the energy of the electrons at temperature $T$ and the second term their energy before thermal excitation. Making use of the fact that $f_{n}=1-f_{-n}$ and that the number of excited electrons is equal to the number of holes left behind, one gets:

$$
\Delta \varepsilon \sum_{n=1,2 .} \rho\left(\varepsilon_{n}\right) f_{n}=\Delta \varepsilon \sum_{n=1,2 .} \rho\left(\varepsilon_{-n}\right)\left(1-f_{-n}\right) .
$$


Using

$$
\varepsilon_{n}-\varepsilon_{\mathrm{F}}=\left(n-\frac{1}{2}\right) \Delta \varepsilon, \quad \varepsilon_{-n}-\varepsilon_{\mathrm{F}}=-\left(n-\frac{1}{2}\right) \Delta \varepsilon
$$

one obtains:

$$
\Delta U=2 \rho\left(\varepsilon_{\mathrm{F}}\right)(\Delta \varepsilon)^{2} \sum_{n=1,2 . .} \frac{\left(n-\frac{1}{2}\right)}{\exp \left\{\left(n-\frac{1}{2}\right) \Delta \varepsilon / k T\right\}+1} .
$$

The electronic specific heat follows immediately

$$
C_{e}=2 \rho\left(\varepsilon_{\mathrm{F}}\right)(\Delta \varepsilon)^{2} \sum_{n=1,2 . .} \frac{\partial}{\partial T} \frac{\left(n-\frac{1}{2}\right)}{\exp \left\{\left(n-\frac{1}{2}\right) \Delta \varepsilon / k T\right\}+1} .
$$

Fröhlich discusses two limiting cases of eq. (3.13): $\Delta \varepsilon \ll k T$ and $\Delta \varepsilon \gg k T$. Looking at our estimates, the first condition is fulfilled for a macroscopic piece of metal for $T \simeq 1 \mathrm{~K}$.

Because the Fermi function of eq. (3.13) does not change significantly in the interval $\Delta \varepsilon / k T$, in the high temperature limit, the summation can be replaced by an integral. Introducing the variable $\xi=\left(n-\frac{1}{2}\right) \Delta \varepsilon / k T$, and putting $\partial / \partial T=(\partial \xi / \partial T) \partial / \partial \xi=-\left(\left(n-\frac{1}{2}\right) \Delta \varepsilon / k T^{2}\right) \partial / \partial \xi$ one obtains:

$$
C_{\mathrm{e}}=-2 \rho\left(\varepsilon_{\mathrm{F}}\right) k^{2} T \int_{0}^{\infty} \xi^{2} \frac{\partial}{\partial \xi} \frac{1}{e^{\xi}+1} \mathrm{~d} \xi=\frac{\pi^{2}}{3} \rho\left(\varepsilon_{\mathrm{F}}\right) k^{2} T
$$

which is the well known result obtained from the continuous level distribution $\rho(\varepsilon)$ [80]. Qualitatively speaking, the familiar $T$-law arises because at $\varepsilon_{\mathrm{F}}$ a very large number of states $\varepsilon_{n}$ is contained in the energy interval $k T$ : the weighting Fermi function distributes the electrons as if the level distributions were continuous.

The situation is different when $\Delta \varepsilon \gg k T$; if $\Delta \varepsilon$ is large, the Fermi function will not lift a single electron in the first excited state. Noticing that only the term with $n=1$ contributes significantly in eq. (3.13), one obtains

$$
C_{\mathrm{e}}=2 \rho\left(\varepsilon_{\mathrm{F}}\right)(\Delta \varepsilon)^{2} \frac{\partial}{\partial T} \frac{\frac{1}{2}}{\mathrm{e}^{\Delta \varepsilon / 2 k T}+1} \simeq \frac{1}{2} \rho\left(\varepsilon_{\mathrm{F}}\right) \frac{(\Delta \varepsilon)^{3}}{k T^{2}} \mathrm{e}^{-\Delta \varepsilon / 2 k T} .
$$

The specific heat decreases exponentially with decreasing temperature for a well defined and given $\Delta \varepsilon$. This is the typical illustration for the quantum size effect. Fig. 3.1 taken from Fröhlich's [2] original paper, shows the ratio $\gamma=C_{\mathrm{e}} / C_{\infty}$ as a function of the parameter $k T / \Delta \varepsilon ; C_{\infty}$ is the electronic specific heat of the bulk metal. As can be seen, $\gamma$ depends on the position of $\varepsilon_{\mathrm{F}} \cdot \gamma_{\mathrm{a}}$ corresponds to the case where the Fermi level lies halfway between two neighbouring electron levels; $\gamma_{\mathrm{b}}$ applies to the case when $\varepsilon_{\mathrm{F}}$ coincides with an electron level; $\gamma=\frac{1}{2}\left(\gamma_{\mathrm{a}}+\gamma_{\mathrm{b}}\right)$ is the average specific heat one expects for an assembly of metal particles with Fröhlich's level structure and well defined $\Delta \varepsilon$ but varying position of $\varepsilon_{\mathrm{F}}$.

The results obtained so far need some discussion:

(1) What are the conditions to make the QSE parameter $x=\Delta \varepsilon / k T$ sufficiently big $(x \gg 1)$ ? 


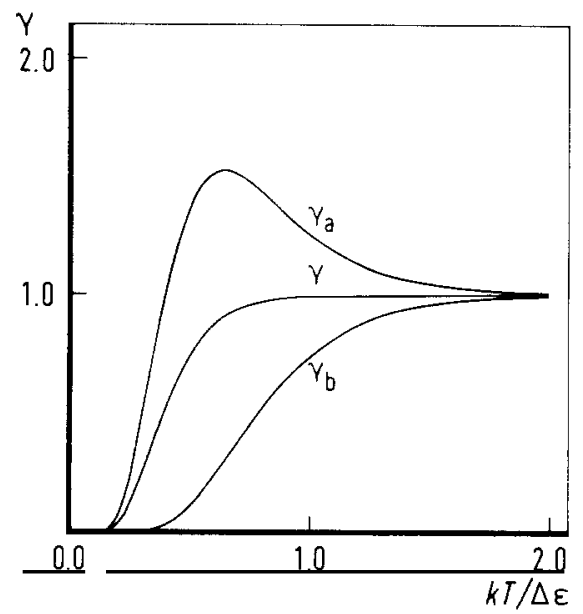

Fig. 3.1. The ratio $\gamma=C_{\mathrm{e}} / C_{\infty}$ of the electronic heat capacity $C_{\mathrm{e}}$ of the small metallic particle to the heat capacity $C_{\infty}$ of the bulk metal as a function of temperature, calculated for the case of equal level spacing with a level spacing $\Delta \varepsilon$. For the calculation of $\gamma_{\mathrm{a}}$, the Fermi level was positioned in the middle between two levels, for $\gamma_{b}$, the Fermi level coincided with one of the electron levels, and finally $\gamma=\frac{1}{2}\left(\gamma_{a}+\gamma_{b}\right)$. (From Fröhlich [2].)

Obviously, for a given temperature, the size of the cube has to be sufficiently reduced; in Fröhlich's model, according to eq. (3.4), one has $\Delta \varepsilon \sim 1 / L^{2}$. On the other hand, for a given $L$ the condition can also be fulfilled when the temperature is low enough. For temperatures of the order of $T \simeq 1 \mathrm{~K}, L$ must be rather small, of the order of $10 \mathrm{~nm}$ : therefore the saying that QSE occur only for very small objects. However, one has to bear in mind that not $L$ itself is the decisive quantity, but $x=\Delta \varepsilon / k T$, hence $x \sim(1 / k T)\left(1 / L^{2}\right)$.

(2) The high degeneracy of the levels (given by $\rho\left(\varepsilon_{n}\right) \Delta \varepsilon$ ) and the constant spacing $\Delta \varepsilon$ between the levels are consequences of the very simple but also very unrealistic model which was chosen. Even in the framework of the free electron model, the actual distribution of the levels $\varepsilon_{n}$ will reflect the boundary conditions imposed by the shape of the particle; as the latter consists of discrete atoms, the perfect cube will never be realized in practice. How this influences the electronic orbitals is a very difficult and yet unsolved problem. Different approaches to its solution will be presented in the subsequent sections. Already here it is worthwhile to note that the $1 / L^{2}$-dependence of $\Delta \varepsilon$ is an artefact of the level degeneracy of Fröhlich's model. It will be shown that in most cases the average level spacing is given by $\Delta \varepsilon \sim 1 / V \sim 1 / L^{3}$.

(3) Specific surface properties have been neglected, although it is well known that surface states exist which are characterized by their finite extension into the interior of the crystal. At present, surface phenomena are part of active research and to incorporate surface properties into the small particle problem leads to immense complications.

(4) The electronic levels are considered to be perfectly sharp, this implies an infinite lifetime of the excited states. In bulk solids, different scattering mechanisms are responsible for broadening of the energy levels. The finite spacing $\Delta \varepsilon$ between neighbouring levels in the QSE regime makes the scattering less effective than in bulk. However, the broadening due to scattering has to be small compared with $\Delta \varepsilon$, otherwise no manifestations of the discreteness of the energy spectrum can be expected. 


\subsection{Kubo's small particle}

\subsubsection{General considerations and assumptions}

As in Fröhlich's treatment, the fundamental fact that the spacing between adjacent levels increases with decreasing particle size lies at the basis of Kubo's treatment of the electronic properties of small particles [3]. However, the artificial concept of equally spaced levels is abandoned and a number of new, realistic assumptions is made.

The most important point concerns the level scheme. Kubo emphasizes the irregularity of the particle shape which does not justify the boundary conditions of the model of perfect cubes as used by Fröhlich. As the shape of the particles is not known down to details of atomic dimensions, Kubo replaces the degenerate level scheme of eq. (3.3) by a statistical distribution. Here, use is made of an important result on the influence of the boundary conditions on the asymptotic density of eigenvalues of the wave equation, a problem treated in a number of papers by $\mathrm{H}$. Weyl $[81,82]$ (for a recent review, see refs. $[83,84])$. There it is shown that the density of eigenvalues with large indices - states with high "quantum numbers", in the quantum mechanical language - does not depend on the shape of the region for which the wave equation is solved; similarly, it does not depend on the exact nature of the imposed boundary conditions. The density of eigenvalues with large quantum numbers is a function of the volume of the region considered alone. The question arises what the measure is to call a quantum number 'big' or 'small'. Although exact and explicit prescriptions are given in the literature to answer this question [85], in the following it is taken for granted that the quantum number of the Fermi level is so high that these asymptotic theorems can be applied. Consequently, over energy intervals which are large compared with the spacing between adjacent levels, the density of states $\rho(E)$ does not depend on the boundary conditions at all. Therefore, one uses e.g. periodic boundary conditions and one obtains [86]

$$
\rho\left(\varepsilon_{\mathrm{F}}\right)=\frac{3}{2} N / \varepsilon_{\mathrm{F}}
$$

$N$ is the number of electrons contained in the particle. The energy levels contributing to eq. (3.16) are spin degenerate.

As one is interested not only in the 'coarse grained' density of states but in the positions of the individual levels as well, a distribution of the individual levels must be introduced such that $\rho\left(\varepsilon_{\mathrm{F}}\right)$ of eq. (3.16) is conserved. This was done by Fröhlich by assuming an equal level spacing and a high degeneracy. Kubo takes a more realistic approach in postulating that the levels are randomly distributed. This means that the energy axis is divided in intervals small compared to

$$
\delta=2 / \rho\left(\varepsilon_{\mathrm{F}}\right)=\frac{4}{3} \varepsilon_{\mathrm{F}} / N
$$

where $\delta$ is the average spacing between two levels which are only spin degenerate. Each interval has the same, small probability of containing a level. Then, according to elementary statistics, the spacing $\Delta$ between adjacent levels is Poisson distributed:

$$
P(\Delta)=\frac{1}{\delta} \mathrm{e}^{-\Delta / \delta}
$$

$P(\Delta) \mathrm{d} \Delta$ is the probability of finding the nearest level in the interval $(\Delta, \Delta+\mathrm{d} \Delta)$ from the level considered, see fig. 3.2. Such a distribution is qualitatively different from the equal level spacing with 


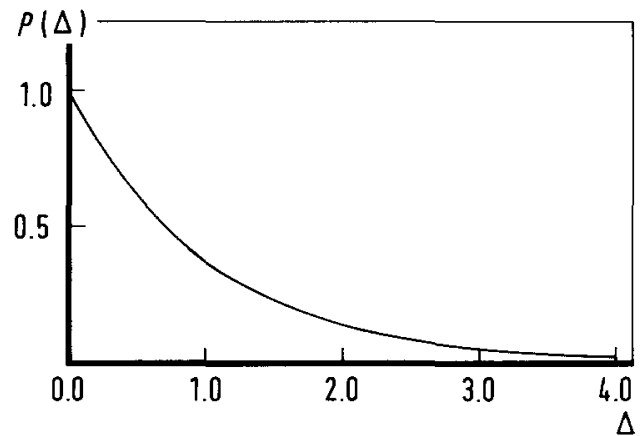

Fig. 3.2. The probability distribution $P(\Delta)$ of the spacings $\Delta$ between the levels for a random (Poisson-)distribution of the single electron energy levels.

constant $\Delta \varepsilon$ in Fröhlich's model. The same is true for thermodynamical properties derived from this model as shown in the next subsection.

The difference between $\delta_{\mathrm{F}}=\Delta \varepsilon \sim 1 / L^{2}$ and $\delta_{\mathrm{K}} \sim 1 / L^{3}$ can be demonstrated by a numerical example. For a cubic particle of gold (with an electron concentration of $5.9 \times 10^{28} \mathrm{~m}^{-3}$ ) with $10 \mathrm{~nm}$ side length and containing about $6 \times 10^{4}$ atoms, one gets for the average level separation according to Kubo $\delta_{\mathrm{K}}=$ $0.6 \times 10^{-4} \mathrm{eV}=0.7 \mathrm{~K}$, whereas for Fröhlich's model, one gets $\delta_{\mathrm{F}}=3.6 \times 10^{-3} \mathrm{eV}=42 \mathrm{~K}$.

It is useful to note that $P(\Delta)$ is largest for $\Delta=0$. This means that for a random arrangement the levels are 'attracting' each other, leading to accidental, not explicitly intended degeneracy. There is a simple argument against introducing a priori any degeneracy of the levels. Degeneracy is always connected to symmetry; if the symmetry is due to geometry, the geometrical form of the particles should be perfect down to structural details of the order of the de Broglie wavelength of the electrons at $\varepsilon_{\mathrm{F}}$, which is about $0.1 \mathrm{~nm}$. With an exception for organo-metallic clusters (which are in fact well defined molecules) it is not reasonable to assume, that any real particles can be made symmetric to this extent.

A further innovation due to Kubo is the concept of particles containing an even or an odd number of electrons. This concept is based on pure electrostatics: the energy needed to charge a sphere of diameter $d$ with a charge $e$ is in vacuum $e^{2} / 2 \pi \varepsilon_{0} d$. The charge can only originate from the surrounding medium (i.e. the heat reservoir) which is at temperature $T$. Therefore, when $k T\left(\simeq 10^{-4} \mathrm{eV}\right.$ at $1 \mathrm{~K}) \ll e^{2} / 2 \pi \varepsilon_{0} d\left(\simeq 10^{-1} \mathrm{eV}\right.$ for $\left.d=20 \mathrm{~nm}\right)$, the probability for an electron to be captured by a particle is very small, charge fluctuations are highly improbable. As the particle is in its lowest energy state, when the total charge equals zero, the assumption is made, that at low temperature the particles are electrically neutral.

Because of the improbability of charge fluctuations, there will be particles containing an even number of electrons and particles containing an odd number of electrons, and it will turn out that in these two cases different properties are found. The even/odd distinction has originally been put forward by Kubo and has subsequently been adopted in most small particle work.

\subsubsection{Electron specific heat and Pauli spin paramagnetism}

In principle, the method for the calculation of the thermodynamic properties of Kubo's small particles is straightforward. Nevertheless, the typical small particle properties mentioned in the preceding paragraph necessitate some precautions.

The charge conservation (i.e. the existence of particles with an even and an odd number of electrons) complicates the computation considerably, because all quantities have to be derived from the particle 
conserving canonical ensemble; it will turn out that the "odd-particle" properties are markedly different from the "even-particle" ones in the QSE regime. Therefore, the normally used grand canonical ensemble must be discarded as it contains fluctuations of the number of electrons. In the case of a degenerate Fermi gas the fluctuations are given by [87]:

$$
\overline{(\Delta N)^{2}}=\frac{m k T}{\pi \hbar^{2}}\left(\frac{3}{\pi} \frac{N}{V}\right)^{1 / 3} V
$$

For a particle with linear dimensions of $10 \mathrm{~nm}$ and an electron density of $10^{29} \mathrm{~m}^{-3}$, one has $(\Delta N)^{2} \simeq 1$, which is not very much compared to the total number of electrons of $N \simeq 10^{5}$. However, contrary to the normal situation in statistical mechanics, in the present case not the relative fluctuation $(\Delta N)^{2} / N$ is important, but the absolute change of $N$ by one electron. Stated in a different way: It does not matter that the particle contains 10000 or 10002 electrons, but it matters that it contains 10000 or 10001 electrons. The necessity to abandon the grand canonical ensemble entails annoying computational consequences. The most important consequence is the fact that the Fermi function is no longer a valid expression for the occupation probability of the single electron states.

Furthermore, the level distribution of eq. (3.18) must be taken into account which leads to inconvenient averaging processes, even when all particles are of the same size. When the particles vary in size, an additional averaging over the size distribution is required, each size characterized by its proper value of $\delta$ derived from eq. (3.17).

In Appendix I an outline of Kubo's calculation is presented, emphasizing some critical steps. Here, we give only the main results including some discussion.

In the high-temperature limit $(\delta / k T \ll 1)$, one gets for the specific heat:

$$
C_{V}=\frac{1}{3} \pi^{2} k^{2} T \rho\left(\varepsilon_{\mathrm{F}}\right) \quad(\delta / k T \ll 1)
$$

and for the susceptibility due to the Pauli spin paramagnetism:

$$
\chi=\mu_{0} \mu_{\mathrm{B}}^{2} \rho\left(\varepsilon_{\mathrm{F}}\right) \quad(\delta / k T \ll 1)
$$

These are just the familiar results for the properties of a bulk metal. When the thermal energy $k T$ is much bigger than the average level spacing $\delta$, a large number of levels above the Fermi energy are occupied and therefore many energies contribute to the partition function. Consequently, a large number of configurations have to be taken into account for the computation of the partition function, and a negligible error is introduced when the summation is replaced by an integration. As long as the mean level spacing does not change, all level distributions give the same results for the thermodynamic quantities; this is exactly the customary bulk situation where the level structure enters only in the form of the density of states at $\varepsilon_{\mathrm{F}}$.

In the low temperature limit $(\delta / k T \gg 1)$, only very few levels above $\varepsilon_{\mathrm{F}}$ will be occupied, of the order of 2 or 3 . The partition function will then depend crucially on the position of the few individual levels around $\varepsilon_{\mathrm{F}}$; therefore, not only the average spacing $\delta=2 / \rho\left(\varepsilon_{\mathrm{F}}\right)$ is important but also the distribution of the first few single particle levels around $\varepsilon_{\mathrm{F}}$. In addition, a different behaviour will be seen for particles with an odd and an even number of electrons ("odd" and "even" case) due to charge-conservation. [3]:

As shown in Appendix I, one gets for the specific heat in zero magnetic field the Kubo-QSE results 


$$
\begin{aligned}
& C_{\text {odd }}=1.645 k^{2} T \rho\left(\varepsilon_{\mathrm{F}}\right) \\
& C_{\text {even }}=2.512 k^{2} T \rho\left(\varepsilon_{\mathrm{F}}\right) . \quad(\delta / k T \gg 1, \quad H=0)
\end{aligned}
$$

In the low field limit, the magnetization is given by:

$$
\begin{aligned}
& M_{\text {odd }}=\mu_{\mathrm{B}} \tanh \left(\mu_{0} \mu_{\mathrm{B}} H / k T\right) \\
& M_{\text {even }}=4 \mu_{\mathrm{B}}(k T / \delta) \sinh \left(2 \mu_{0} \mu_{\mathrm{B}} H / k T\right) \int_{0}^{1} \frac{\mathrm{d} t}{1+4 t \cosh ^{2}\left(\mu_{0} \mu_{\mathrm{B}} H / k T\right)+t^{2}} .
\end{aligned}
$$

$\left(\delta / k T \gg 1, \mu_{0} \mu_{\mathrm{B}} H / \delta \ll 1\right)$

For $H \rightarrow 0$, one gets for the spin susceptibility:

$$
\begin{aligned}
& \chi_{\text {odd }}=\mu_{0} \mu_{\mathrm{B}}^{2} / k T \\
& \chi_{\text {even }}=1.521 \mu_{0} \mu_{\mathrm{B}}^{2} \rho\left(\varepsilon_{\mathrm{F}}\right) .
\end{aligned}
$$

Finally, the magnetization in the QSE- and the high field-limit is given by:

$$
\begin{aligned}
& M_{\text {odd }}=2 \mu_{0} \mu_{\mathrm{B}}^{2} H / \delta+\frac{1}{2} \mu_{\mathrm{B}}\left(1+\exp \left(-4 \mu_{0} \mu_{\mathrm{B}} H / \delta\right)\right) \\
& M_{\text {cven }}=2 \mu_{0} \mu_{\mathrm{B}}^{2} H / \delta+\frac{1}{2} \mu_{\mathrm{B}}\left(1-\exp \left(-4 \mu_{0} \mu_{\mathrm{B}} H / \delta\right)\right)
\end{aligned}
$$

Contrary to Fröhlich's result of eq. (3.15), the electron specific heat for a Poisson distributed level scheme is a linear function of the temperature. Compared with the bulk material, its value is reduced by about one third. More dramatic new features are found for the Pauli spin paramagnetism. The odd particles behave at low temperatures as if the magnetic properties were determined by the spin of a free electron occupying a state which for $H=0$ is only spin degenerate. This leads to the Curie-type temperature dependence of the magnetization as given by eq. (3.24a). It is interesting to note that $\chi_{\text {even }}$ does not vanish, even when $\delta / k T \rightarrow \infty$. This is a consequence of the Poisson level scheme where levels "attract" each other: the smaller the level spacing the bigger the probability of its occurrence; therefore, for any value of $\delta / k T$ there is always a nonzero probability to find levels with energy-distance $\Delta<k T$. We shall see in the next section that $\chi_{\text {even }}(T)$ is totally different if level distributions are used where the levels repel rather than attract each other, i.e. distributions where the probability for very small level spacings $\Delta \rightarrow 0$ goes to zero.

The importance of the canonical calculation instead of the customary grand canonical one has been emphasized by Denton, Mühlschlegel and Scalapino [88]; they have calculated the electronic heat capacity for Fröhlich's equal level spacing case using Fröhlich's method (i.e. the grand canonical ensemble) and the canonical ensemble. As is shown in Appendix II it is possible to calculate the canonical partition function exactly for the equal level spacing case. The results of Denton, Mühlschlegel and Scalapino are shown in fig. 3.3.

Most important for experimental verifications of the theoretical QSE predictions is a more precise specification of the condition $\delta / k T \gg 1$. As higher order analytical calculations to check the influence of the $(\delta / k T)$-term are very tedious in the case of the Poisson level scheme, Denton, Mühlschlegel and 

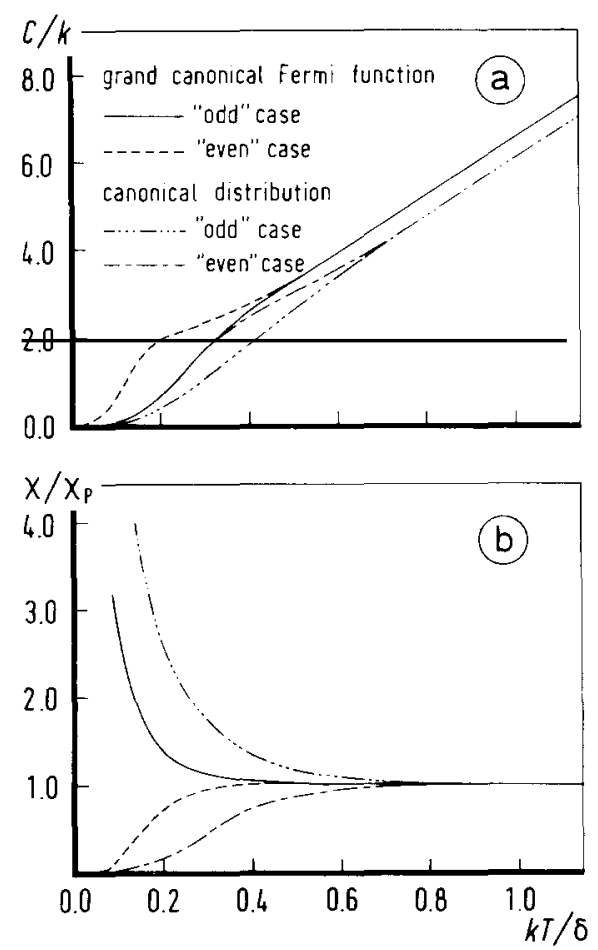

Fig. 3.3. (a) The electronic heat capacity $C$, and (b) the spin susceptibility $\chi$ as a function of temperature for a particle with an equal spacing $\delta$ between the levels, calculated using the grand canonical ensemble and the electron number conserving canonical ensemble, respectively. $\chi$ is normalized to the Pauli spin paramagnetic susceptibility $\chi_{\mathrm{P}}$. (From Denton, Mühlschlegel and Scalapino [88].)

Scalapino made computer calculations. It turns out that the lowest order approximation, containing only terms of the order $(\delta / k T)^{-1}$, is valid only when the condition $\delta / k T \geq 10$ is fulfilled.

\subsection{Level statistics}

In the QSE limit, the thermodynamic properties of the electrons are determined by two fundamental concepts: the single electron level structure and charge conservation. The example of the specific heat showed that by assuming an equal spacing between degenerate levels, one gets in the limit of small $k T / \delta$ an exponential $T$-dependence (eq. (3.15)), whereas Kubo's random distribution of energy levels gives the familiar linear $T$-law (eqs. (3.22a), (3.22b)) as for the bulk metal, although with smaller coefficients. In addition, fig. 3.3 shows how charge conservation influences the specific heat and the spin susceptibility for a particle with equal level spacing $\delta$.

A wide range of different possibilities for the level distribution can be offered. Fröhlich's equal level spacing is probably an oversimplification of the actual situation; Kubo's Poisson scheme is very plausible but it is in fact an "ad hoc" assumption. Gor'kov and Eliashberg [4] have pointed out that there are distributions which are possibly more justified. These distributions will be discussed in the following, relying very much on a paper by Porter and Rosenzweig [89, 90].

\subsubsection{An analogy with statistical mechanics}

Statistical mechanics describes the global behaviour of systems which consist of a large number of 
'particles'-e.g. a gas of atoms in a macroscopic volume. Because of the large number of atoms, the exact time development of the system cannot be determined, although the interaction between the atoms is quite well understood. The statistics give information about the global, average properties of the system, like pressure or temperature. This is accomplished by making some basic assumptions on the occupation probability of phase space. "A priori" probabilities are attributed to all states which are accessible to the system. The justification of this procedure lies mainly in its success.

In this subsection we would like to point out that there is a close analogue between ordinary statistical mechanics and level statistics, when roughly speaking - the roles of the "known" and the "unknown" quantities of statistical mechanics are interchanged.

To be more specific, let us consider a small metallic particle. The electron-like excitations in this particle form a degenerate Fermi gas, they interact with each other, with other sorts of excitations, and with the walls of the particle. As explained in section 3.2, this latter interaction is of very complicated nature; therefore, the Hamiltonian of the electron system will be very complicated. The problem consists now in finding the energy spectrum of this Hamiltonian, i.e. the stationary states in the single electron approximation.

The lowest states of the system can be found with sufficient accuracy if the real Hamiltonian is substituted by a reasonable approximation for which the eigenvalue equation can be solved. However, for the high energies of interest here (around $\varepsilon_{\mathrm{F}}$ ), already small perturbations have to be taken into account, influencing the spectrum more and more with increasing energy. As these small perturbations are complicated and not known, the eigenfunctions of the complicated Hamiltonian cannot be calculated. Therefore, one introduces the concept of level statistics in the hope that it gives information on global properties of the spectrum, such as the probability distribution of the energy eigenvalues.

This illustrates the meaning of interchanging "known" and "unknown" quantities when going from statistical mechanics to level statistics.

In ordinary statistical mechanics the "unknown" quantity is the state of the system and the "known" quantities are the forces acting on the atoms. For a closed system each phase space element of given volume-corresponding to a state of the total system - is given the same a priori probability of being occupied when the state is compatible with the energy of the system. Clearly, each volume element in phase space is equivalent to a possible state of the system. Such an assumption is certainly inadequate if we want to get knowledge on the level structure of a complex system because it already anticipates what in fact has to be looked for. In level statistics the role of the "unknown" quantities is played by the forces: It is explicitly assumed that the Hamiltonian of the system is so complex, that its single terms cannot be enumerated. This complex system is thought of as a system in which many particles are interacting according to unknown laws. Subsequently an ensemble of systems is considered in which all laws of interaction, compatible with certain general symmetry properties, are given equal a priori probability.

In table 3.1 a comparison is made between the usual statistical mechanics and the new technique of level statistics. This concept of energy level statistics based on an ensemble of systems is due to Wigner [91]. The hope, that such a statistical procedure for finding the probability distribution of the energy levels may be successful, is (as in the usual statistical mechanics) based on the expectation, that the system might be described by a huge variety of complex Hamiltonians, but that the spectral properties of these Hamiltonians will not deviate very much from the spectral properties of a properly chosen average Hamiltonian for the ensemble [92]. The exceptions will be formed by systems with a high degree of symmetry (i.e. simple systems). In these systems, the matrix elements of the Hamiltonian are strongly correlated and their interdependence is most clearly shown by group theory. However, when 
Table 3.1

Comparison of the level statistics with the usual statistical mechanics

\begin{tabular}{|c|c|}
\hline Statistical mechanics & Level statistics \\
\hline State $\{p, q\}$ of the system not known. & Interaction between the constituents of the system not known. \\
\hline $\begin{array}{l}\text { Statistical assumption on the distribution of states and } \\
\text { their occupation in phase space. }\end{array}$ & $\begin{array}{l}\text { Statistical assumption on the interactions (of certain } \\
\text { symmetry type). }\end{array}$ \\
\hline $\begin{array}{l}\text { The hope is, that overall properties of the system can } \\
\text { be found: } p, T, \ldots\end{array}$ & $\begin{array}{l}\text { The hope is, that overall properties of the level spectrum of } \\
\text { the system can be found, especially the probability } \\
\text { distribution of energy eigenvalues. }\end{array}$ \\
\hline
\end{tabular}

e.g. the states of a system can be separated in categories with even and odd parity, it can be shown that for each of these two categories alone the theory of level statistics might give very good results, but when the densities of states are compared without taking parity into account, no agreement is found. This means that deviations of an actual level distribution from the ensemble average suggest the existence of conserved quantum numbers which have been overlooked.

\subsubsection{Symmetry properties of the Hamiltonian}

As we have discussed in great length, the interactions of an electron in a small particle are very complicated. The detailed form of the Hamiltonian $H$ can in general not be determined to such an extent to give the energy spectrum around $\varepsilon_{\mathrm{F}}$ with sufficient precision. Nevertheless, the Hamiltonian exists, and may have some symmetry properties. In this section we will study these symmetry properties of the Hamiltonian in some detail. In Hilbert space, the Hamiltonian can be represented by a hermitian matrix $H$ :

$$
H=H^{\dagger}=\tilde{H}^{\star}
$$

$H^{\dagger}$ is the hermitian-conjugated matrix, obtained by complex conjugation of the transposed matrix $\tilde{H}$.

If there is no time-reversal invariance, then nothing is known about the Hamiltonian matrix $H$, except that it must be a complex hermitian matrix. This matrix can be written down numerically in any representation, differing from any other, by a unitary transformation $U$ :

$$
U^{\dagger} U=I, \quad \text { or } U^{\dagger}=U^{-1}
$$

Beside hermiticity, some other, well known, general invariance properties of the Hamiltonian can be considered. They are listed in table 3.2. Space translation invariance leads to the familiar conservation of momentum, and time translation invariance to conservation of energy. Our problem is to analyse the structure of the Hamiltonian under the various combinations of the remaining symmetry properties.

Let us consider time inversion invariance. The time reversal operator $T$ is an anti-unitary operator, and can be written as the product of a unitary operator $U$ and the complex conjugation operator $K$ : $T=U K$. When no spin dependent terms come into the Hamiltonian, a basis can be chosen, so that $U$ is just the unity operator:

$$
T=K, \quad T^{2}=K^{2}=I
$$


Table 3.2

General symmetry properties of the Hamiltonian

\begin{tabular}{ll}
\hline Invariance & Associated operator \\
\hline Space translation & Total momentum \\
Time translation & Total energy \\
Space inversion & Parity \\
Time inversion & Time reversal \\
Space rotation & Total angular momentum \\
\hline
\end{tabular}

When the spin enters, the choice of $U$ is dictated by the properties of the total angular momentum operator $\boldsymbol{J}$. From the requirement $T \boldsymbol{J} T^{-1}=-\boldsymbol{J}$, one can deduce, that an appropriate choice of $T$ is:

$$
\begin{aligned}
& T=\mathrm{i} \sigma_{y} K=\exp \left(\mathrm{i} \pi S_{y} / \hbar\right) K \\
& T^{2}=\mathrm{i} \sigma_{y} K \mathrm{i} \sigma_{y} K=\left(\begin{array}{rr}
0 & 1 \\
-1 & 0
\end{array}\right)\left(\begin{array}{rr}
0 & 1 \\
-1 & 0
\end{array}\right) K^{2}=-I
\end{aligned}
$$

here $\boldsymbol{S}$ is the spin operator and $\sigma_{y}$ a Pauli spin matrix. For $N$ spins:

$$
\begin{aligned}
& T=\exp \left(\mathrm{i} \pi \sum S_{n y} / \hbar\right) K \\
& T^{2}=+I, \quad \text { for even } N \\
& T^{2}=-I, \quad \text { for odd } N .
\end{aligned}
$$

When $T^{2}=+I, U$ can be diagonalized; a basis can be chosen, so that, again, $T=K$ [92].

Time invariance, together with the hermiticity of $H$, gives:

$$
\left.\begin{array}{l}
H=T H T^{-1}=H^{\star} \\
H=H^{\dagger}=\tilde{H}^{\star}
\end{array}\right\} H=H^{\star}, \quad \text { and } H=\tilde{H}
$$

the Hamiltonian is therefore real and symmetric. This symmetry property of $H$ is conserved under an orthogonal transformation $O$, with

$$
\tilde{O} O=I, \quad \text { or } \tilde{O}=O^{-1} .
$$

When $T^{2}=-I$, we note, that for a state $\Psi$ satisfying the Schrödinger equation:

$$
(\Psi, T \Psi)=\left(T \Psi, T^{2} \Psi\right)^{\star}=-(T \Psi, \Psi)^{\star}=-(\Psi, T \Psi)
$$

or

$$
(\Psi, T \Psi)=0
$$

When the Hamiltonian is time inversion invariant, both $\Psi$ and $T \Psi$ satisfy the Schrödinger equation for 
the same energy. Therefore, the degeneracy is at least twofold. This is the famous Kramers degeneracy. Due to this spin degeneracy, the matrix $T$ cannot be diagonalized; the appropriate transformations are the $2 N \times 2 N$ matrices from the $N$-dimensional symplectic group $\operatorname{Sp}(N)$. In this case, the Hamiltonian is a quaternion-real matrix.

However, when the Hamiltonian is rotationally invariant (even in the case $T^{2}=-I$ ), an alternative definition of the time reversal operator is possible:

$$
T^{0}=\exp \left(-\mathrm{i} \pi J_{y} / \hbar\right) T=\exp \left(-\mathrm{i} \pi J_{y} / \hbar\right) \exp \left(\mathrm{i} \pi S_{y} / \hbar\right) K=\exp \left(-\mathrm{i} \pi L_{y} / \hbar\right) K
$$

and now $\left(T^{0}\right)^{2}=+I$ again, therefore, $U$ can be diagonalized. Just as in the case, where only orbital angular momenta were considered, the orthogonal transformations are the symmetry operations for the Hamiltonian.

The different types of symmetry, discussed above, are summarized in table 3.3. These symmetry properties of the Hamiltonian may have interesting consequences for the eigenvalue spectrum.

Let us consider the accidental approach of two eigenvalues of the Hamiltonian; we will determine the probability $P(\Delta)$, that the spacing between these levels is $\Delta$ [93-95]. For the orthogonal ensemble the relevant part of the Hamiltonian (which is real and symmetric in this case) is:

$$
H=\left(\begin{array}{rr}
x & y \\
y & -x
\end{array}\right)
$$

the eigenvalue equation can be solved directly using the secular equation:

$$
\left|\begin{array}{cc}
x-E & y \\
y & -x-E
\end{array}\right|=0
$$

and we find as eigenvalues for $H$ :

$$
E= \pm \sqrt{x^{2}+y^{2}}
$$

the probability to find a nearest level spacing in the interval between $\Delta$ and $\Delta+\mathrm{d} \Delta$ is just the volume in the parameter space $(x, y)$ for which $E$ lies in the interval between $\frac{1}{2} \Delta$ and $\frac{1}{2} \Delta+\frac{1}{2} \mathrm{~d} \Delta$, this is $\frac{1}{2} \pi \Delta \mathrm{d} \Delta$; therefore

$$
P(\Delta) \mathrm{d} \Delta \sim \Delta \mathrm{d} \Delta
$$

Table 3.3

The symmetry and transformational properties of the Hamiltonian

\begin{tabular}{lll}
\hline Symmetry & Hamiltonian & Canonical group \\
\hline No time inversion symmetry. & Hermitian. & Unitary transformations. \\
Time inversion symmetry, integral spin. & Real, symmetric. & Orthogonal transformations. \\
Time inversion symmetry, space rotation symmetry. & Real, symmetric. & Orthogonal transformations. \\
Time inversion symmetry, half-integral spin. & Quaternion real. & Symplectic transformations. \\
\hline
\end{tabular}


Similarly, for the unitary ensemble, the matrix $H$ is hermitian:

$$
H=\left(\begin{array}{cc}
x & y^{\prime}+\mathrm{i} y^{\prime \prime} \\
y^{\prime}-\mathrm{i} y^{\prime \prime} & -x
\end{array}\right)
$$

and the eigenvalues of this Hamiltonian are:

$$
E= \pm \sqrt{x^{2}+y^{\prime 2}+y^{\prime \prime 2}}
$$

in this case the volume in the parameter space $\left(x, y^{\prime}, y^{\prime \prime}\right)$, for which the nearest level distance $2 E$ lies between $\Delta$ and $\Delta+\mathrm{d} \Delta$, is proportional to $\Delta^{2} \mathrm{~d} \Delta$,

$$
P(\Delta) \mathrm{d} \Delta \sim \Delta^{2} \mathrm{~d} \Delta
$$

Finally, for the symplectic ensemble, the Hamiltonian is represented by a quaternion-real matrix; the relevant part, which describes the interaction of two Kramers doublets, approaching in energy, is given by:

$$
H=\left(\begin{array}{cccc}
x & 0 & y & \mathrm{i} z \\
0 & x & \mathrm{i} z^{\star} & y^{\star} \\
y^{\star} & z & -x & 0 \\
-\mathrm{i} z^{\star} & y & 0 & -x
\end{array}\right)
$$

where $y=y^{\prime}+\mathrm{i} y^{\prime \prime}$, and $z=z^{\prime}+\mathrm{i} z^{\prime \prime}$, the solution of the secular equation is:

$$
E= \pm \sqrt{x^{2}+y y^{\star}+z z^{\star}}
$$

this leads to a spacing probability:

$$
P(\Delta) \mathrm{d} \Delta \sim \Delta^{4} \mathrm{~d} \Delta .
$$

The most spectacular feature of the results in eqs. (3.36), (3.38) and (3.40), is the vanishing probability of the occurrence of very small level spacings. Contrary to Kubo's distribution, eq. (3.18), the chance of an accidental degeneracy of two levels is zero. In the next section, we will consider the level spacing distribution for the different ensembles in more detail.

\subsubsection{The orthogonal ensemble}

As we have discussed above, the orthogonal ensemble is appropriate when

(1) The Hamiltonian $H$ is time inversion invariant;

(2) a. The angular momentum $J / \hbar$ is integer, or

b. The Hamiltonian is rotation invariant.

In the limiting case of small spin orbit coupling and when no applied magnetic field is present (which would disturb the time inversion symmetry), the electron spin is conserved. The spin merely gives a twofold degeneracy of the states. The angular momentum entering the Hamiltonian is then integer, and condition $2 \mathrm{a}$ is fulfilled. Therefore, as shown before, $H$ is a real, symmetric matrix, and the appropriate 
basis transformations are the orthogonal matrices $O$, with

$$
\tilde{O} O=I, \quad \text { or } \tilde{O}=O^{-1}
$$

As explained in section 3.3.1, some reasonable assumptions on the matrix elements have to be made, based on these symmetry properties of $H$. A suitable probability distribution for the matrix elements is obtained when equal weight is given to all possible interactions. From this distribution, one finds the probability distribution of the eigenvalues, which solves the problem in principle.

In a first assumption, one considers a finite $N \times N$ matrix in place of the exact infinite dimensional matrix $H_{\alpha \beta}$; the distribution of eigenvalues of the finite matrix is supposed to be as representative for the actual system as the one of the infinite matrix. This assumption will be discussed at the end of this section. The other two basic assumptions concern the probability with which a matrix occurs, where the matrix elements $H_{\alpha \beta}(\alpha=1, \ldots N ; \beta=1, \ldots N)$ have certain numerical values. Because $H$ is a symmetric matrix, it consists of $N(N+1) / 2$ independent elements. The probability, that the first element lies in the interval $\left(H_{11}, H_{11}+\mathrm{d} H_{11}\right)$, the second in the interval $\left(H_{12}, H_{12}+\mathrm{d} H_{12}\right)$ and so on, is given by $P_{N}\left(H_{11}, H_{12}, \ldots, H_{N N}\right) \mathrm{d} H_{11} \mathrm{~d} H_{12} \ldots \mathrm{d} H_{N N}$. The differential probability $P_{N}\left(H_{11}, H_{12}, \ldots H_{N N}\right) \equiv$ $P_{N}\left(H_{i j}\right)$ must be constructed in such a way that it satisfies the fundamental requirements of

(1) independence of the matrix element from the chosen basis;

(2) invariance of $P_{N}\left(H_{i j}\right)$ when a transition from one real basis to an other real basis is made.

These requirements are sufficient to give an explicit analytic determination of $P_{N}\left(H_{i j}\right)$. They give the mathematical formulation of the statistical properties of a system subject to random forces with a special type of symmetry.

The independence of the matrix elements leads to factorization:

$$
\begin{aligned}
& P_{N}\left(H_{i j}\right)=f_{11}\left(H_{11}\right) f_{12}\left(H_{12}\right) \ldots f_{\alpha \beta}\left(H_{\alpha \beta}\right) \ldots f_{N N}\left(H_{N N}\right) \\
& (\alpha \leq \beta)
\end{aligned}
$$

The transformation from one real basis to another real basis is given by a real unitary (i.e. orthogonal) matrix $O$. Therefore, when putting

$$
H^{\prime}=O^{-1} H O
$$

$P_{N}\left(H_{i j}\right)$ must satisfy

$$
P_{N}\left(H_{i j}^{\prime}\right)=P_{N}\left(H_{i j}\right)
$$

Eqs. (3.42) and (3.44) determine $P_{N}\left(H_{i j}\right)$, the straightforward calculation is shown explicitly in ref. [89]. One finds that the matrix elements $H_{\alpha \beta}$ (with $\alpha \neq \beta$ ) and $H_{\gamma \gamma}$ are normally distributed, the dispersion of the $H_{\gamma \gamma}$ being twice the dispersion of the $H_{\alpha \beta}$ :

$$
P_{N}\left(H_{i j}\right)=C \exp \left(-\left(H_{11}^{2}+2 H_{12}^{2}+\cdots+H_{N N}^{2}\right) / 4 \sigma^{2}\right)=C \exp \left(-\operatorname{Tr} H^{2} / 4 \sigma^{2}\right) .
$$

$\sigma^{2}$ is the mean square dispersion of the off-diagonal elements.

As a next step, one has to diagonalize the "random matrix" $H_{i j}$ in order to obtain the distribution function for the eigenvalues $E_{\lambda} . \sigma$ will have the meaning of a scaling factor for the energy difference 
between the $E_{\lambda}$. The transformation from the basis $\left|\psi_{\alpha}\right\rangle$ to the eigenvectors $\left|\chi_{\lambda}\right\rangle$ of the unknown Hamiltonian $H$ is accomplished by the orthogonal transformation $\Lambda\left(a_{\mu \nu}\right)$. For $N^{2}$ elements $a_{\mu \nu}$, there are $N(N+1) / 2$ normalization and orthogonality constraints. Therefore, each $a_{\mu \nu}$ depends on $N(N-$ 1)/2 independent parameters $\alpha_{i}$ :

$$
a_{\mu \nu}=a_{\mu \nu}\left(\alpha_{1}, \alpha_{2}, \ldots, \alpha_{N(N-1) / 2}\right)
$$

We try to obtain the eigenvalue distribution by replacing the $N(N+1) / 2$ values $H_{\alpha \beta}$ in eq. (3.45) by $N$ values $E_{\lambda}$ (defined by $H\left|\chi_{\lambda}\right\rangle=E_{\lambda}\left|\chi_{\lambda}\right\rangle$ ) and $N(N-1) / 2$ values $\alpha_{i}$. Again from simple orthogonality arguments, it follows that

$$
\sum_{\mu, \xi} H_{\mu \xi}^{2}=\sum_{\mu} E_{\mu}^{2}
$$

The differential $\mathrm{d} H_{11} \ldots \mathrm{d} H_{N N}$ has to be replaced by $J_{N} \mathrm{~d} E_{1} \ldots \mathrm{d} E_{N} \mathrm{~d} \alpha_{1} \ldots \mathrm{d} \alpha_{N(N-1) / 2}$ where $J_{N}$ is the Jacobian of the coordinate transformation:

$$
J_{N}=\left|\begin{array}{ccc}
\frac{\partial H_{11}}{\partial E_{1}} & \frac{\partial H_{11}}{\partial E_{2}} \cdots \frac{\partial H_{11}}{\partial \alpha_{N(N-1) / 2}} \\
\vdots & \vdots \\
\frac{\partial H_{N N}}{\partial E_{1}} & \frac{\partial H_{N N}}{\partial E_{2}} \cdots \frac{\partial H_{N N}}{\partial \alpha_{N(N-1) / 2}}
\end{array}\right|
$$

The Jacobian (see ref. [89]) can be separated into two factors, one containing the $E_{\lambda}$ only and the other containing the $\alpha_{i}$ :

$$
J_{N}=\left[\prod_{\mu<\nu}\left|E_{\mu}-E_{\nu}\right|\right] h\left(\alpha_{1}, \alpha_{2}, \ldots, \alpha_{N(N-1) / 2}\right)
$$

By integrating over the independent parameters $\alpha_{i}$ one obtains after substituting eqs. (3.47) and (3.49) in eq. (3.45):

$$
P_{N}\left(E_{1}, E_{2}, \ldots, E_{N}\right)=C\left[\prod_{\mu<\nu}\left|E_{\mu}-E_{\nu}\right|\right] \exp \left(-\frac{1}{4 \sigma^{2}} \sum_{\lambda=1}^{N} E_{\lambda}^{2}\right)
$$

where $C$ is a constant of normalization. This expression, known as Wishart distribution [96], is the result we have been looking for: The probability distribution of the eigenvalues of a symmetric random matrix, characterizing the Hamiltonian of a "random system" subject to statistically equally probable interactions with a certain symmetry. For $N=2$, eq. (3.50) reduces to

$$
P_{2}\left(E_{1}, E_{2}\right)=C_{2}\left|E_{1}-E_{2}\right| \exp \left[-\left(E_{1}^{2}+E_{2}^{2}\right) / 4 \sigma^{2}\right]
$$

From this expression it is easy to derive the distribution for the level spacings $P_{2}^{0}(\Delta)$. (The superscript 0 indicates that we consider spacings between adjacent levels only, with zero levels in between. For $N=2$, there are of course no other spacings.) With the definition for the level spacing $\Delta=E_{1}-E_{2}>0$, 
and for the mean eigenvalue $\bar{E}=\frac{1}{2}\left(E_{1}+E_{2}\right)$, eq. (3.51) can be written as:

$$
P_{2}(\Delta, \bar{E})=C_{2}^{\prime} \Delta \exp \left(-\Delta^{2} / 8 \sigma^{2}\right) \exp \left(-\bar{E}^{2} / 2 \sigma^{2}\right) \text {. }
$$

Integrating over all $\bar{E}$ leads to:

$$
P_{2}(\Delta)=C_{2}^{\prime \prime} \Delta \exp \left(-\Delta^{2} / 8 \sigma^{2}\right)
$$

The mean level spacing $\delta$ is now given by:

$$
\delta=\int_{0}^{\infty} \Delta P_{2}(\Delta) \mathrm{d} \Delta
$$

Introducing the variable $x=\Delta / \delta$, replacing the normalization constant $C_{2}^{\prime \prime}$ by its actual value [97] and making use of the relation $\sigma=\delta / \sqrt{2 \pi}$, one gets for the spacing distribution:

$$
P_{2}^{0}(x)=\frac{1}{2} \pi x \exp \left(-\frac{1}{4} \pi x^{2}\right) .
$$

This distribution (3.55) is also known under the name of Wigner surmise because it was supposed by Wigner that the nearest level spacing of the orthogonal ensemble should be distributed according to this law. However, this assumption is correct only for $N=2$; for higher dimensions it is no longer true. Nevertheless, for all practical purposes, the Wigner surmise is an excellent approximation (even for $N \rightarrow \infty$ ) so that the exact nearest neighbour distributions are only of academic interest [98].

For higher dimensions $(N>2)$, one is not only interested in the nearest neighbour spacings $P_{N}^{0}(x)$, but also in the next-nearest spacings $P_{N}^{1}(x)$, next-next-nearest spacings $P_{N}^{2}(x)$, and so on. The eigenvalues $E_{i}$ must first be ordered according to magnitude. With increasing dimensionality, the mathematical difficulties soon become enormous and machine computations must replace analytic brainwork. In fig. 3.4a the Wigner surmise $P_{2}^{0}$ is shown, together with the Poisson distribution for comparison. The absence of small level spacings is more pronounced for the unitary and symplectic ensemble, in accordance with the results of eqs. (3.36), (3.38) and (3.40). In fig. 3.4b the machine calculated distributions are shown for the nearest level spacing $P_{10}^{0}$, the next-nearest level spacing $P_{10}^{1}$, the next-next-nearest level spacing $P_{10}^{2}$, up to $P_{10}^{5}$; the calculation was done for a $10 \times 10$ random matrix [99].

The level repulsion, shown to arise from this model, has been observed in the nearest level spacings taken from spectroscopic data of complex atoms and nuclei $[89,100]$.

\subsubsection{The unitary and symplectic ensemble}

When the spin enters the Hamiltonian explicitly through spin orbit coupling, the symplectic ensemble is the appropriate ensemble. The spin orbit coupling must be strong enough to mix up levels which would have been determined by the orthogonal ensemble previously.

A criterion to estimate the strength of the spin orbit coupling has been given by Kawabata [12]. The symplectic ensemble applies when

$$
\left(\hbar v_{\mathrm{F}} / d\right)(\Delta g)^{2} \gg \delta
$$



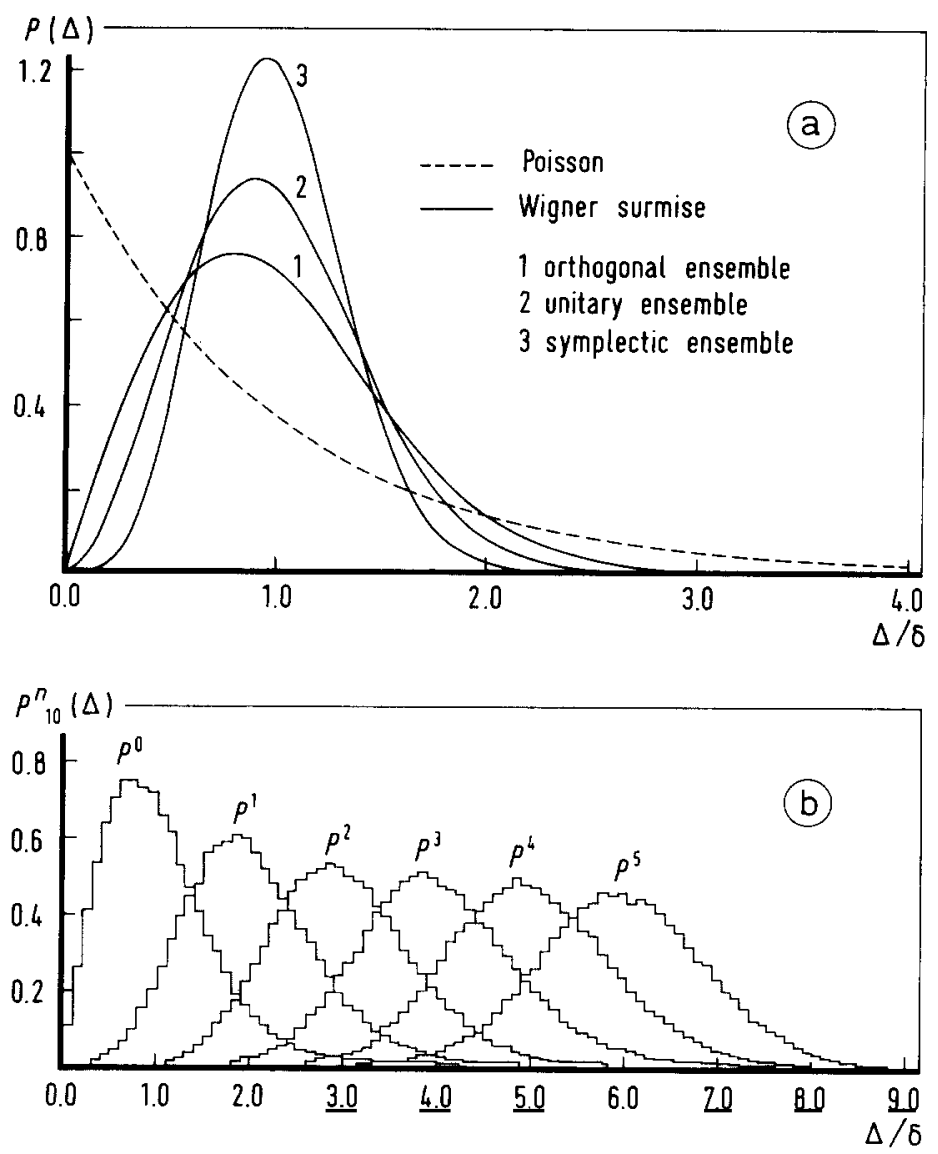

Fig. 3.4. (a) Comparison of nearest level spacing distributions: the Poisson distribution and the Wigner surmise $P_{2}^{0}$ for the orthogonal, the unitary, and the symplectic ensemble. (b) Computer calculated distributions for the nearest level spacing distribution $P_{10}^{0}$, the next-nearest level spacing distribution $P_{10}^{1}$, etc., for a system described by a $10 \times 10$ random matrix. (From Porter [99].)

$v_{\mathrm{F}}$ is the Fermi velocity of the electrons, $d$ is the particle diameter, and $\Delta g$ is the electronic $g$-shift as measured by an electron spin resonance (ESR) experiment. This Kawabata-rule is quite plausible: The ESR line width is according to Elliott [101] given by:

$$
1 / \tau \simeq(\Delta g)^{2} / \tau_{\mathrm{tr}}
$$

where $\tau_{\mathrm{tr}}$ is the relaxation time entering the DC electrical conductivity. For small particles, where the electronic mean free path $l$ is bigger than the dimensions of the particle $(l>d), \tau$ is given by $\tau_{\mathrm{tr}} \simeq d / v_{\mathrm{F}}$. The electron energy is determined within $\Delta E \simeq \hbar / \tau$; when $\Delta E \gg \delta$, "mixing of the levels" occurs and the symplectic ensemble is appropriate. Conversely, for $\left(\hbar v_{\mathrm{F}} / d\right)(\Delta g)^{2} \ll \delta$ the orthogonal ensemble must be used.

When the appropriate symmetry transformations from the symplectic group are taken into account, the level distribution found is:

$$
P_{N}\left(E_{1}, E_{2}, \ldots, E_{N}\right)=C\left[\prod_{\mu<\nu}\left|E_{\mu}-E_{\nu}\right|^{4}\right] \exp \left(-\frac{1}{4 \sigma^{2}} \sum_{\lambda=1}^{N} E_{\lambda}^{2}\right)
$$


The Wigner surmise for the nearest level spacings for the symplectic ensemble is of the form:

$$
P_{2}^{0}(x)=\frac{2^{18}}{3^{6} \pi^{3}} x^{4} \exp \left(-\frac{64}{9 \pi} x^{2}\right)
$$

In this case the level repulsion is drastically stronger than in the case of the orthogonal ensemble, because of the factor $\left|E_{\mu}-E_{\nu}\right|^{4}$. The presence of the Kramers degeneracy seems to make any additional degeneracy enormously more unlikely [92].

Furthermore, time reversal symmetry is broken by a magnetic field. If in the presence of spin orbit coupling the magnetic field is sufficiently strong $\left(\mu_{0} \mu_{\mathrm{B}} H>\delta\right.$, see ref. [4]), the unitary ensemble applies instead of the symplectic one. It's probability distribution is given by:

$$
P_{N}\left(E_{1}, E_{2}, \ldots, E_{N}\right)=C\left[\prod_{\mu<\nu}\left|E_{\mu}-E_{\nu}\right|^{2}\right] \exp \left(-\frac{1}{4 \sigma^{2}} \sum_{\lambda=1}^{N} E_{\lambda}^{2}\right)
$$

and the Wigner surmise is

$$
P_{2}^{0}(x)=\frac{32}{\pi^{2}} x^{2} \exp \left(-\frac{4}{\pi} x^{2}\right)
$$

\subsubsection{Physical consequences of the repulsion of energy levels}

In the QSE limit, the repulsion of energy levels leads to drastic effects in the behaviour of the susceptibility of particles with an even number of electrons. In Kubo's model, the spin susceptibility in the "even case" is only weakly dependent on the temperature, and at low temperatures it is slightly bigger than the familiar Pauli spin susceptibility (see eq. (3.24b)). This behaviour is due to the accidental degeneracy of the Poisson level distribution, eq. (3.18). When this degeneracy is lifted and $\mu_{0} \mu_{\mathrm{B}} H<\delta$, then the particles with an even number of electrons will not contribute to the magnetization at all as all the spin-up and spin-down levels are occupied up to $\varepsilon_{\mathrm{F}}$ and the higher levels are inaccessible at low temperature $(k T<\delta)$ and in low magnetic fields. Therefore one expects that the spin susceptibility in the "even case" should vanish when repulsion of the energy levels occurs.

As discussed in the preceding sections, the appropriate random ensemble is selected by the symmetry properties of the system under consideration:

1. The orthogonal ensemble can be used when the Hamiltonian is time inversion invariant and the total angular momentum is integer. These conditions are fulfilled for low magnetic fields $\left(\mu_{0} \mu_{\mathrm{B}} H \ll \delta\right)$ and weak spin orbit coupling $\left(\left(\hbar v_{\mathrm{F}} / d\right)(\Delta g)^{2} \ll \delta\right)$.

2. The symplectic ensemble can be used when the Hamiltonian is time inversion invariant as well, and when the total angular momentum is half integer.

3. The unitary ensemble must be used when the Hamiltonian is not time inversion invariant.

These different cases again reflect the old distinction between "even" and "odd" particles. When the spin does not enter the Hamiltonian explicitly, as it is the case for weak spin orbit coupling, the orthogonal ensemble applies. However, when the spin orbit coupling is so strong that spin dependent terms have to be taken into account, even and odd particles behave quite differently: For even particles the total angular momentum is again integer, and the orthogonal ensemble may be used; for odd particles, with half integer total angular momentum, the symplectic ensemble with its very strong level 
repulsion applies. If time reversal symmetry is broken by a strong magnetic field, the distinction between even and odd particles loses its significance and then the unitary ensemble is appropriate.

Czerwonko derived asymptotic formulas for the specific heat and spin susceptibility for the various random ensembles in the high temperature $(k T \gg \delta)$ and low temperature $(k T \ll \delta)$ limit [102-104]. Results valid over the whole temperature range have been obtained by Denton, Mühlschlegel and Scalapino $[88,105]$ using an ingenious approximative method. They give an exact result for the partition function of a particle with equally spaced single electron levels (see Appendix II); using this equal level spacing case as a zeroth approximation for level repulsion, they proceed to better approximations by allowing the levels next to $\varepsilon_{\mathrm{F}}$ to vary according to distribution functions of the type of eq. (3.55). As a next step, the next-nearest level spacing distribution can be introduced, and so on. From the distribution functions $P_{N}^{n}(\Delta)$ in fig. 3.4b a quick convergence of this procedure can be expected. In figs. 3.5 and 3.6 the spin susceptibility and specific heat are shown, calculated as a function of $k T / \delta$ [88]. In fig. 3.5 the vanishing of the spin susceptibility in even particles comes out clearly. The dominant contribution to the susceptibility of the odd particles stems from the alignment of the one unpaired spin.

The low temperature behaviour of the specific heat can be derived very easily [4,93-95]. The free energy $F$ can be found from the partition function $Z$, as a simple average over the appropriate ensemble. For low temperatures $(k T \ll \delta)$ only the lowest excitations need to be considered. The

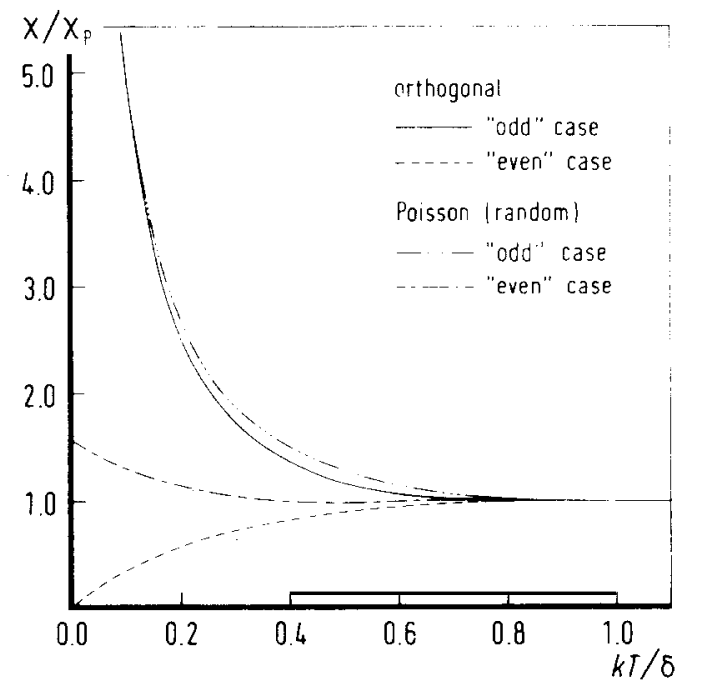

Fig. 3.5. The spin susceptibility $\chi$ of a small metallic particle normalized to the Pauli spin paramagnetic susceptibility $\chi_{\mathrm{P}}$ as a function of temperature. The average level spacing is $\delta$, and for the levels around $\varepsilon_{\mathrm{F}}$ an averaging over the orthogonal or over the Poisson level probability distribution was carried out. (From Denton. Mühlschlegel and Scalapino [88].)

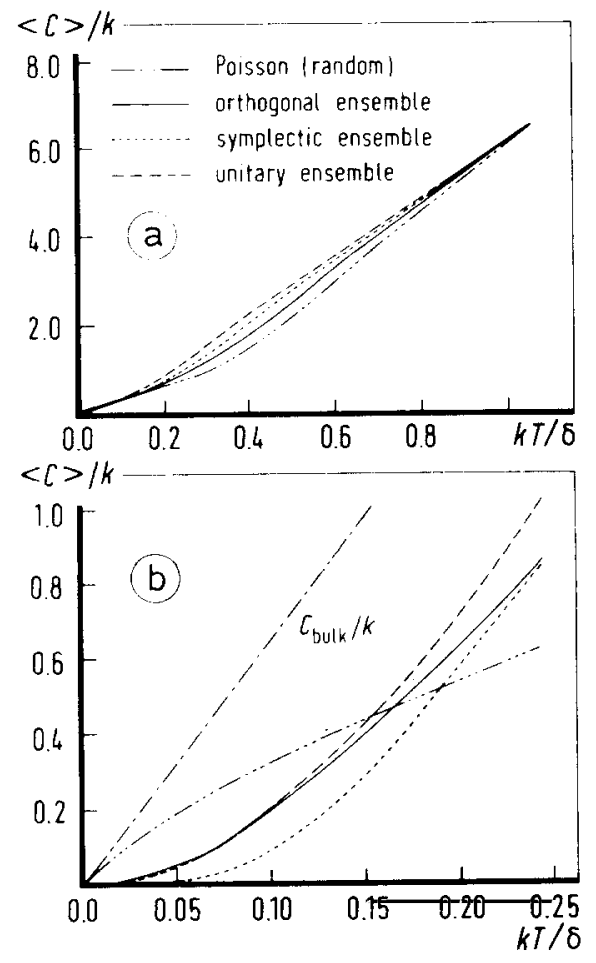

Fig. 3.6. (a) The electronic specific heat of a small metallic particle as a function of temperature. The average level spacing is $\delta$ and for the levels around $\varepsilon_{\mathrm{F}}$ an averaging over the level probability distribution was carried out. (b) A blow up of the QSE region, $k T / \delta<0.25$. (From Denton, Mühlschlegel and Scalapino [88].) 
partition function for odd and even particles is then simply:

$$
\begin{array}{ll}
Z=2+4 \mathrm{e}^{-\Delta / k T} & \text { for odd particles } \\
Z=1+4 \mathrm{e}^{-\Delta / k T} & \text { for even particles }
\end{array}
$$

With the low-energy approximation for the level spacing distribution $P(\Delta)$ as given by eqs. (3.36), (3.38) and (3.40) we can easily carry out the ensemble average. The specific heat is then given by the expression

$$
C=T \frac{\partial^{2}}{\partial T^{2}} k T \int_{0}^{\infty} P(\Delta) \ln Z \mathrm{~d} \Delta
$$

from which we obtain $C \sim T^{2}$ for the orthogonal ensemble, $C \sim T^{5}$ for the symplectic ensemble, and $C \sim T^{3}$ for the unitary ensemble, in accordance with the low temperature approximations obtained by other authors $[88,102-105]$. The decrease of the specific heat with temperature is stronger for the larger level repulsion effect.

\subsection{Spectroscopy of the small particle level structure}

Probably the most direct method to probe the level structure of small particles is single electron excitation with optical methods. If $\delta / k$ is of the order of $1 \mathrm{~K}$, the low lying excitations correspond to radiation in the far infrared $\left(1 \mathrm{~K} \simeq 0.7 \mathrm{~cm}^{-1}\right)$. Theoretically, the behaviour of small size- $h$ quantized metallic particles exposed to an electromagnetic field has been studied by Gor'kov and Eliashberg [4].

Consider first a particle in a static applied electric field $\boldsymbol{E}_{0}$. Due to the discreteness of the level spectrum, a weak applied field $\left(e d E_{0}<\delta\right)$ is not able to induce a current as it is possible in a bulk metal where a shielding charge distribution is built up. In a small metallic particle, the electric field will cause a distortion of the electronic wave functions in such a way that charge is accumulated on one side of the particle and removed from the other side. The particle behaves like a dielectric or as a giant molecule. For small fields $E_{0}$, first order perturbation theory can be applied and the polarization of the particle will be a linear function of the applied field.

Gor'kov and Eliashberg assumed that the internal field $\boldsymbol{E}_{\text {int }}$ inside the particle is constant. This is certainly not correct for very small particles with a low electron density because the screening length becomes of the order of the particle dimension, as was discussed by Rice, Schneider and Strässler [106]. However, when the internal field $E_{\text {int }}$ is constant, one may set $\boldsymbol{E}_{\text {int }}=\boldsymbol{E}_{0}-L \boldsymbol{P} / \varepsilon_{0}$, where $L$ is the depolarization factor and $\boldsymbol{P}$ the net polarization of the particle, defined as $p / V$ with $\boldsymbol{p}$ the induced electric dipole moment (cf. eq. (2.17)) and $V$ the volume of the particle. The electric susceptibility $\chi$ and the polarizability $\alpha$ are defined by:

$$
\begin{aligned}
& \boldsymbol{P}=\chi \varepsilon_{0} \boldsymbol{E}_{\mathrm{int}}=(\varepsilon-1) \varepsilon_{0} \boldsymbol{E}_{\mathrm{int}} \\
& \boldsymbol{p}=\alpha \varepsilon_{0} \boldsymbol{E}_{0}
\end{aligned}
$$

(as discussed in section 2.2, for the case of a particle embedded in a medium with dielectric constant $\varepsilon_{\mathrm{m}}$ we must substitute $\left.\chi_{\mathrm{eff}}=\left(\varepsilon-\varepsilon_{\mathrm{m}}\right) / \varepsilon_{\mathrm{m}}\right)$. Obviously, $\alpha$ and $\chi$ are connected by the simple relation 


$$
\alpha=\frac{\chi}{1+L \chi} V
$$

It should be emphasized that, contrary to what is stated in ref. [4], Gor'kov and Eliashberg have calculated the susceptibility $\chi$ and not the polarizability $\alpha$ [107].

The frequency dependent susceptibility of a small particle can be calculated by using a generalization of the usual method to calculate the polarizability of the hydrogen atom in its ground state (see e.g. ref. [108]):

$$
\alpha(\omega)=\frac{2 e^{2}}{\varepsilon_{0}} \sum_{k} \frac{E_{k}-E_{0}}{\left(E_{k}-E_{0}\right)^{2}-\hbar^{2} \omega^{2}}\left|x_{0 k}\right|^{2} .
$$

Here, the electric field is assumed to be along the $x$-direction. For the hydrogen atom one has, as usual, $\boldsymbol{E}_{\text {int }}=\boldsymbol{E}_{0}$; however, in the case of small particles, this has to be corrected: eq. (3.66) gives the response of the electrons to the electric field which they experience (i.e. $\boldsymbol{E}_{\text {int }}$ for a small particle) and therefore $\alpha(\omega)$ has to be replaced by $\chi(\omega)$. As there are many electrons involved in a small particle, occupation probabilities $n_{k}$ have to be introduced for the states $|k\rangle$. Single particle absorption occurs at energies $\hbar \omega_{k l}=\left(E_{k}-E_{l}\right)$; damping can be introduced in a phenomenological way by a complex frequency notation $\omega \rightarrow \omega+\mathrm{i} \Gamma$. The absorption of the system is then given by the imaginary part of $\chi(\omega)$. With the substitution $\left|x_{k l}\right|^{2}=\frac{1}{3}\left|r_{k l}\right|^{2}$, one gets for the susceptibility of a small particle:

$$
\chi(\omega)=\frac{e^{2}}{3 \varepsilon_{0}} \sum_{k, l} \frac{\left(E_{k}-E_{l}\right)\left(n_{k}-n_{l}\right)}{\left(E_{k}-E_{l}\right)^{2}-\hbar^{2}(\omega+\mathrm{i} \Gamma)^{2}}\left|\boldsymbol{r}_{k l}\right|^{2} .
$$

Separating the static and dynamic part leads to the following result:

$$
\begin{aligned}
& \chi(0)=\frac{e^{2}}{3 \varepsilon_{0}} \sum_{k, l} \frac{n_{k}-n_{l}}{E_{k}-E_{l}}\left|r_{k l}\right|^{2} \\
& \chi(\omega)-\chi(0)=-\frac{e^{2} \hbar^{2} \omega^{2}}{3 \varepsilon_{0}} \sum_{k, l} \frac{n_{k}-n_{l}}{E_{k}-E_{l}} \frac{\left|r_{k l}\right|^{2}}{\left(E_{k}-E_{l}\right)^{2}-\hbar^{2}(\omega+i \Gamma)^{2}}
\end{aligned}
$$

Following Shapoval, the matrix elements $\left|\boldsymbol{r}_{k l}\right|^{2}$ can be evaluated using a quasi-classical method [109]. As this method is of rather general interest, we will sketch it briefly. The expectation values of the quantity $\langle\boldsymbol{r}(t) \boldsymbol{r}(t+\tau)\rangle_{n i}$ are calculated in the state $|n i\rangle ; \boldsymbol{r}(t)$ is the position operator and $|n i\rangle$ denotes a state of energy $E_{n}$, the label $i$ will take into account possible degeneracy. Changing over to the Schrödinger representation with a time independent operator $r$, one obtains

$$
\langle\boldsymbol{r}(t) \boldsymbol{r}(t+\tau)\rangle_{n i}=\sum_{m} \exp \left(-\mathrm{i}\left(E_{n}-E_{m}\right) \tau / \hbar\right) \sum_{j}\langle n i|\boldsymbol{r}| m j\rangle\langle m j|\boldsymbol{r}| n i\rangle .
$$

With $\hbar \omega_{n m}=E_{n}-E_{m}$ and $\Sigma_{j}\langle n i|r| m j\rangle\langle m j|r| n i\rangle=2\left|r_{n m}\right|^{2}$ (taking into account the spin degeneracy of the orbital state $|n i\rangle)$ this relation can be written as:

$$
\langle\boldsymbol{r}(t) r(t+\tau)\rangle_{n i}=2 \sum_{m} \exp \left(-\mathrm{i} \omega_{n m} \tau\right)\left|r_{n m}\right|^{2}=\frac{2}{\delta} \int_{-\infty}^{\infty} \exp (-\mathrm{i} \omega \tau)\left|r_{n m}(\omega)\right|^{2} \mathrm{~d} \omega
$$


As $\langle\boldsymbol{r}(t) \boldsymbol{r}(t+\tau)\rangle_{n i}$ is an even function of $\tau$, the Fourier inversion leads to:

$$
\left|r_{m n}(\omega)\right|^{2}=\frac{\delta}{2} \frac{1}{2 \pi} 2 \int_{0}^{\infty}\langle r(t) r(t+\tau)\rangle_{n i} \cos (\omega \tau) \mathrm{d} \tau
$$

At this point, a quasi-classical approximation is made: The fact is being used that the mean expectation value of any operator averaged over all states with a certain energy can be approximated by the average of the classical expectation values evaluated along trajectories of the particle with a given energy. In eq. (3.71) the index $n i$ will be replaced by $E_{n}$ and the average is taken over the classical trajectories of energy $E_{n}:$ A straight line from one point at the surface of the particle to another. The electron is then supposed to be scattered diffusely off the surface (otherwise the quasi-classical approximation cannot be used, see ref. [109]). The time between two scattering events at the surface is typically $\tau \simeq d / 2 v_{\mathrm{F}}$. Therefore, the autocorrelation function $\langle\boldsymbol{r}(t) \boldsymbol{r}(t+\tau)\rangle_{E_{n}}$ will vanish after a time of this order of magnitude. The cosine term in eq. (3.71) can be approximated by 1 when the calculation of matrix elements $\left|r_{n m}\right|^{2}$ is restricted to states with $\omega_{n m} \ll v_{\mathrm{F}} / d$. Therefore one gets:

$$
\left|r_{n m}(\omega)\right|^{2}=\left|r_{n m}\right|_{0}^{2}=\frac{\delta}{2 \pi} \int_{0}^{\infty}\langle\boldsymbol{r}(t) \boldsymbol{r}(t+\tau)\rangle_{E_{n}} \mathrm{~d} \tau
$$

Obviously, eq. (3.72) is correct only for low frequencies, $\omega<2 v_{\mathrm{F}} / d \simeq 10^{13} \mathrm{~Hz}$, and for these frequencies $\left|r_{n m}\right|^{2}$ is independent of $\omega$. The evaluation of the integral in eq. (3.72) is tedious but straightforward $[4,109]$, and Gor'kov and Eliashberg obtain

$$
\left|r_{n m}\right|_{0}^{2}=0.0184 \delta d^{3} / \hbar v_{\mathrm{F}}
$$

In order to calculate the dynamic susceptibility of eq. (3.68), Dyson's two level correlation function $R\left(E_{1}, E_{2}\right)$ can be used [92]. $R\left(E_{1}, E_{2}\right) \mathrm{d} E_{1} \mathrm{~d} E_{2}$ gives the probability to find one level $k$ in the interval $\left(E_{1}, E_{1}+\mathrm{d} E_{1}\right)$ and the other level $l$ in the interval $\left(E_{2}, E_{2}+\mathrm{d} E_{2}\right)$ independent of the position of the other levels; $R\left(E_{1}, E_{2}\right)$ is a function of the distance between the energy levels only: $R\left(\left|E_{1}-E_{2}\right|\right)$. Each term in the summation of eq. $(3.68 \mathrm{~b})$ may be multiplied by the probability to find a certain pair of eigenvalues and the summation can then be replaced by an integral. For frequencies $\omega \ll v_{\mathrm{F}} / d$ the susceptibility can be calculated from:

$$
\chi(\omega)=\chi(0)-0.00615 \frac{e^{2} \hbar \omega^{2} d^{3}}{\varepsilon_{0} v_{\mathrm{F}} \delta} \int_{-\infty}^{\infty} \int_{-\infty}^{\infty} \mathrm{d} E_{1} \mathrm{~d} E_{2} \frac{n_{1}-n_{2}}{E_{1}-E_{2}} \frac{R\left(\left|E_{1}-E_{2}\right|\right)}{\left(E_{1}-E_{2}\right)^{2}-\hbar^{2}(\omega+\mathrm{i} \Gamma)^{2}}
$$

For the different ensembles, the functions $R\left(\left|E_{1}-E_{2}\right|\right)$ can be calculated [4, 92], and for the orthogonal ensemble, Dyson gives the following expression:

$$
R(\Delta)=1-\left\{\frac{\sin (\pi \Delta / \delta)}{\pi \Delta / \delta}\right\}^{2}-\frac{\mathrm{d} \sin (\pi \Delta / \delta) /(\pi \Delta / \delta)}{\mathrm{d}(\Delta / \delta)} \int_{\Delta / \delta}^{\infty} \frac{\sin (\pi x)}{\pi x} \mathrm{~d} x
$$


The frequency dependent dielectric susceptibilities are of greatest importance because they reflect the statistical nature of the level distributions via the two level correlation function $R\left(\left|E_{1}-E_{2}\right|\right)$. Gor'kov and Eliashberg have calculated the susceptibility $\chi(\omega)$, which is proportional to the dimensionless function $A(\omega)=2 \hbar^{2} \omega^{2} \rho\left(\varepsilon_{\mathrm{F}}\right) \int\left\{R(\varepsilon) /\left(\varepsilon^{2}-\hbar^{2}(\omega+\mathrm{i} \Gamma)^{2}\right)\right\} \mathrm{d} \varepsilon$. Some errors were detected in the original expressions calculated by Gor'kov and Eliashberg, and very recently Devaty and Sievers [110] derived the following expressions for the real and imaginary parts of this function, $A_{1}$ and $A_{2}$ :

$$
\begin{aligned}
& A_{1}^{\text {orth }}(\eta)=2-\frac{\sin 2 \eta}{\eta}-2 \eta\left[\int_{\eta}^{\infty} \frac{\cos t}{t} \mathrm{~d} t\right] \frac{\mathrm{d}}{\mathrm{d} \eta}\left(\frac{\sin \eta}{\eta}\right) \\
& A_{2}^{\text {orth }}(\eta)=2 \eta-\frac{2 \sin ^{2} \eta}{\eta}+2 \eta\left[\int_{0}^{\eta} \frac{\sin t}{t} \mathrm{~d} t-\frac{\pi}{2}\right] \frac{\mathrm{d}}{\mathrm{d} \eta}\left(\frac{\sin \eta}{\eta}\right) \\
& A_{1}^{\text {unit }}(\eta)=2-\frac{\sin 2 \eta}{\eta} \\
& A_{2}^{\text {unit }}(\eta)=2 \eta-\frac{2 \sin ^{2} \eta}{\eta} \\
& A_{1}^{\text {sympl }}(z)=\frac{2-\frac{\sin 2 z}{2 z}+z\left[\int_{0}^{z} \frac{\sin t}{t} \mathrm{~d} t\right] \frac{\mathrm{d}}{\mathrm{d} z}\left(\frac{\cos z}{z}\right)}{A_{2}^{\text {sympl }}(z)=z-\frac{\sin ^{2} z}{2}+z\left[\int_{0}^{z} \frac{\sin t}{t} \mathrm{~d} t\right] \frac{\mathrm{d}}{\mathrm{d} z}\left(\frac{\sin z}{z}\right)}
\end{aligned}
$$

in these expressions $z=2 \pi \hbar \omega \rho\left(\varepsilon_{\mathrm{F}}\right)$ and $\eta=z / 2 . A_{1}$ and $A_{2}$ are also shown in figs. $3.7 \mathrm{a}, 3.7 \mathrm{~b}$ and $3.7 \mathrm{c}$ as
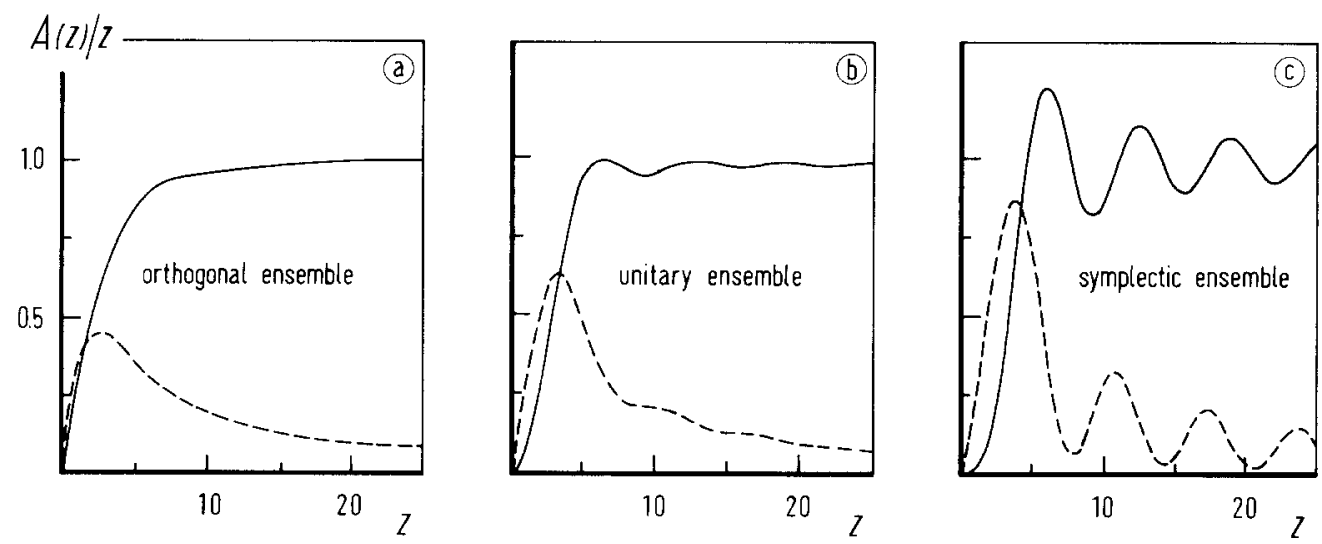

Fig. 3.7. The real and imaginary parts $A_{1}$ (dashed lines) and $A_{2}$ (solid lines) of the dimensionless function $A(z)$. $A(z)$ is proportional to the susceptibility $\chi(\omega)$ characterizing the electrodynamic response of a small metallic particle. The dimensionless variable $z=2 \pi \hbar \omega \rho\left(\varepsilon_{\mathrm{F}}\right)$ is proportional to the energy $\hbar \omega$ of the electromagnetic radiation and the density of states $\rho\left(\varepsilon_{\mathrm{F}}\right)$ at the Fermi level $\varepsilon_{\mathrm{F}}$. The functions $A_{1}$ and $A_{2}$ are shown for the orthogonal (a), the unitary (b), and the symplectic ensemble (c), respectively. For the symplectic ensemble, the susceptibility is a strongly oscillating function of the frequency. (From Devaty and Sievers [110].) 
a function of the variable $z$ for the orthogonal, the unitary, and the symplectic ensemble, respectively. Whereas the absorption, given by $A_{2}$, is a rather smooth function of the frequency for the orthogonal ensemble, the symplectic ensemble gives an absorption with very pronounced oscillations. Their unambiguous experimental verification would constitute a landmark of small particle physics.

\subsection{Experiments}

\subsubsection{Nuclear magnetic resonance (NMR)}

The Knight shift is one of the most elegant tools to study quantum size effects in the spin paramagnetism of the electrons in small particles, because the Knight shift is proportional to the spin susceptibility of the conduction electrons [111] as a result of the contact hyperfine interaction, $\Delta U=a \boldsymbol{I} \cdot \boldsymbol{S} . \boldsymbol{I}$ is the nuclear spin in units of $\hbar$, the hyperfine coupling constant $a$ of the ground state is equal to $\frac{2}{3} \mu_{0} \mu_{\mathrm{B}} \gamma_{\mathrm{N}} \hbar|\Psi(0)|^{2}$, where $\gamma_{\mathrm{N}}$ is the nuclear gyromagnetic ratio and $|\Psi(0)|^{2}$ the density of $s$-like conduction electrons at the position of the nucleus. The spin of the conduction electrons will be aligned in an external magnetic field $B_{0}$, and the net polarization resulting from the Pauli spin paramagnetic susceptibility is given by $\mu_{0} g \mu_{\mathrm{B}}(N / V)\left\langle S_{z}\right\rangle=\chi_{\mathrm{P}} B_{0}$. This leads to a shift of the peak position of the NMR signal. The Knight shift is defined as the fractional change of the resonance field and is therefore given in this case by:

$$
K=-\frac{\Delta B}{B_{0}}=\frac{a \chi_{\mathrm{P}}}{\mu_{0} g \mu_{\mathrm{B}} \overline{\gamma_{N}} \hbar(N / V)}
$$

From eq. (3.77) and the expression for the hyperfine coupling constant given above it is clear that the Knight shift is proportional to the spin susceptibility, and proportional to the ratio of the conduction electron density at the nucleus and the average conduction electron density, $|\Psi(0)|^{2} /(N / V)$. The Knight shift is insensitive to magnetic impurities which may be present at the surface of small particles, in contrast to direct measurements of the susceptibility. Impurities would lead to enhanced spin relaxation and consequently to a broadening of the NMR line, however the position of the resonance would not be influenced.

In a pioneering experiment on QSE Charvolin, Froidevaux, Taupin and Winter reported an investigation of small lithium particles by NMR techniques [112]. The lithium particles were obtained by irradiation of a lithium fluoride crystal with thermal neutrons at $77 \mathrm{~K}$. When the dose of the radiation is properly chosen, two types of lithium particles are formed [113]: Small platelets in the (100) plane of 1 or 2 atomic layers thickness and lateral dimensions in the order of $3 \mathrm{~nm}$, and, simultaneously, thick lithium globules of approximately $30 \mathrm{~nm}$ diameter. The NMR curve recorded at room temperature is shown in fig. 3.8a. Two narrow lines are superposed on a broad resonance. The latter is due to the $\mathrm{Li}^{+}$ of the LiF matrix and is of no further interest for the discussion of QSE. The narrow resonance at lower magnetic fields (labeled " $\mathrm{A}$ " in fig. 3.8a) shows a displacement with respect to the center of the $\mathrm{Li}^{+}$ resonance; the displacement is equal to the Knight shift of bulk lithium metal and this resonance is therefore attributed to the thick lithium globules. The not displaced narrow resonance (labeled " $B$ " in fig. 3.8a) can be explained assuming a QSE. A simple estimate of the average level spacing in the small lithium platelets gives $\delta \simeq 300 \mathrm{~K}$; therefore the occurrence of a QSE is possible, even at room temperature. Obviously, the condition for the low field limit of Kubo's theory (see section 3.2) $\mu_{\mathrm{B}} B<\delta$ is satisfied. Following Kubo, the distinction between even and odd particles is made, but unlike Kubo's assumption of a Poisson level spacing distribution, repulsion of energy levels is thought to occur. Then, 
the spin susceptibility of the "even" particles vanishes in the low field limit, and consequently the Knight shift must be zero in this case which explains the non-shifted resonance "B". In the "odd" case, the spin magnetic moment is due to the one unpaired electron at the Fermi level, for all particles with an odd number of electrons and of all sizes, as long as the QSE condition is fulfilled. The susceptibility for a given particle is constant, whatever its volume, and this implies that the susceptibility per unit volume (of particle) varies with the particle size as $\chi \sim N^{-1}$, where $N$ is the number of atoms per particle. Therefore, each particle with an odd number of electrons has its own size dependent susceptibility $\chi_{0}(d)$. Moreover, when the particles are not of uniform size, one will observe instead of a single resonance the superposition of all the resonances belonging to different values $\chi_{0}(d)$. If the range of particle sizes is wide enough, no resonance will be observed for the "odd" particles, and this seems to be the case in the experiments by Charvolin, Froidevaux, Taupin and Winter. In addition, the fact that the resonance with zero Knight shift disappeared upon annealing of the sample supports the interpretation based on quantum size effects: During the annealing process, the small platelets disappear and much bigger globules are formed. Below room temperature, the NMR experiments become increasingly difficult because the reduced motional narrowing leads to a broadening of the resonance lines. More details on the NMR experiments in small lithium particles can be found in Taupin's papers [114, 115].

Kobayashi, Takahashi, Sasaki and Nomura studied particles of the superconductors aluminium and tin ranging in size from 4.5 to $40 \mathrm{~nm}$ and prepared by evaporation in low pressure helium gas [116-119]; the particles were oxidized to prevent metallic contact. They also prepared very much smaller tin particles (with diameter down to $2.2 \mathrm{~nm}$ ) by evaporation of discontinuous layers of tin with an overlayer of $\mathrm{SiO}_{2}$ [120]. On theoretical grounds, it is expected that the spin pairing in superconductors will lead to a vanishing of the paramagnetic susceptibility of the electrons; however, it is found experimentally that very often the Knight shift does not tend to zero for $T \rightarrow 0$, most probably as a consequence of spin reverse scattering [121]. The shielding of the applied magnetic field due to the Meissner effect prohibits the observation of these effects unless experiments are done on samples with a dimension smaller than the London penetration depth such as small particles (see section 4 for more details). Hines and Knight $[10,122]$ showed that with increase of the spin orbit coupling (by adding suitable impurities), the decrease of the Knight shift was reduced; a decrease of the particle size, causing an enhanced surface scattering rate, has a similar effect. For superconducting aluminium (which is the superconductor with the lowest atomic weight and therefore with the smallest spin orbit coupling) zero Knight shift has been observed for $T \rightarrow 0$ [123]. From a theoretical point of view, Mühlschlegel, Scalapino and Denton [124] have discussed the influence of the QSE on small superconducting particles: Due to the finite size, the phase transition will not be sharp and the spin susceptibility should therefore exhibit a broad transition around $T_{\mathrm{c}}$. When the dimensions of the particles are smaller than the coherence length $\xi$ of the superconducting state, spatially uniform fluctuations of the order parameter will dominate the thermodynamics. For $\delta / k T_{\mathrm{c}} \simeq 1$, they calculated a decrease of the paramagnetic susceptibility at $T_{\mathrm{c}}$ to already $40 \%$ of the bulk value, while $\delta$ should still be considerably bigger to observe quantum size effects. The temperature dependence of the Knight shift for aluminium particles measured by Kobayashi and coworkers [116-118], and Ido and Hoshino [125,126] is in qualitative agreement with these results. Shiba [127] has made a calculation using the equal level spacing model; he has explicitly taken into account spin orbit coupling through mixing of wavefunctions of opposite spin. Unlike the calculations of Denton, Mühlschlegel and Scalapino [105], who calculated the susceptibility using the symplectic ensemble, this model leads to a residual susceptibility at $T=0$ both for superconducting and even normal particles; the magnitude of the residual susceptibility of the electrons depends on the strength of the spin orbit coupling. In recent work Kobayashi and coworkers have measured the Knight 


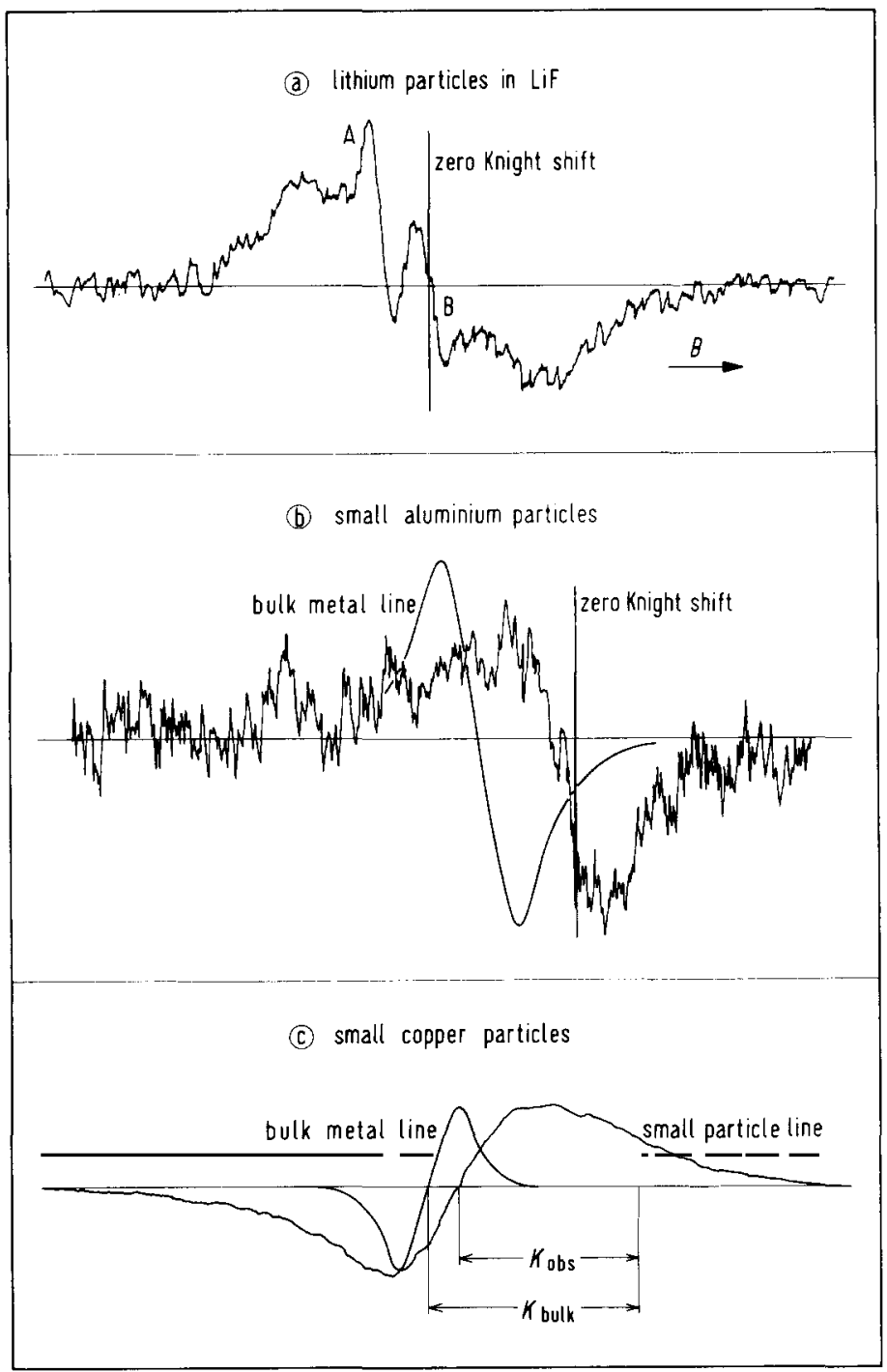

Fig. 3.8. Derivative of the nuclear magnetic resonance signal for small particles of lithium, aluminium and copper. (a) For lithium, two narrow resonances are observed, superposed on the broad resonance of $\mathrm{Li}^{+}$in $\mathrm{LiF}$. The Knight shift of the resonance indicated by " $\mathrm{A}$ " is equal to the bulk value of lithium metal; this resonance is attributed to thick lithium globules. The resonance indicated by "B", with zero Knight shift, is attributed to very small lithium particles containing an even number of electrons for which the susceptibility vanishes because of quantum size effects. (From Charvolin, Froidevaux, Taupin and Winter [112].) (b) The NMR resonance of small gas-evaporated aluminium particles with an average diameter of $4.5 \mathrm{~nm}$. The Knight shift of the small particle resonance is smaller than the Knight shift of the bulk metal. The low field tail is enhanced due to the presence of odd particles of varying size. (From Kobayashi, Takahashi and Sasaki [117].) (c) The NMR resonance of small copper particles with an average diameter of $10 \mathrm{~nm}$, again showing the reduction of the Knight shift $K_{\text {obs }}$ observed in even particles. The particles were prepared by flash evaporation in high vacuum and were isolated with siliconmonoxide. (From Yee and Knight [134].)

shift of aluminium particles in a large applied magnetic field, so that the effects of superconductivity should be largely suppressed. Under these conditions, the Knight shift is found to be substantially reduced as well at low temperatures, indicating a spin pairing which is increasingly more effective as the particle size is reduced [119]. In addition, an indication of the quantum size effect in odd particles was also found: The asymmetry of the resonance line-a tail towards the low field, high Knight shift 
side - was attributed to the particles containing an odd number of electrons. Using a suitable Gaussian distribution of particle sizes, satisfactory agreement between the observed shape and a calculated resonance curve was obtained. For tin, a divalent metal, the resonance line was symmetric, consistent with the absence of odd particles. For tin particles only a small decrease of the Knight shift was found, the residual shift for $T \rightarrow 0$ being larger the smaller their size for particles of diameter down to $10 \mathrm{~nm}$. This is obviously a consequence of the higher spin orbit coupling in tin and the increase of the factor $\hbar / \tau$ with decreasing particle size [118]. For still smaller particles, $d<10 \mathrm{~nm}$, the residual Knight shift decreases with decreasing particle size. This is interpreted as blocking of the mixing of energy levels through spin orbit coupling as a result of QSE [120].

Under quantum size conditions, it is expected that relaxation processes will be blocked. However, no enhancement of the spin relaxation time was observed in small particles: For some samples, even a reduction of the relaxation time $T_{1}$ below the bulk value was noted, indicating the presence of an extra relaxation mechanism probably connected with the presence of paramagnetic impurities at the surface of the particles. The influence of the magnetic field and of the particle size on the relaxation time was studied in detail $[118,120,128]$, and recently a theory was put forward by Sone $[129,130]$ explaining these results. Sone calculated the effects of fluctuations of superconductivity, taking into account the Zeeman energy of the electrons and the depairing effect of the magnetic field, for particles smaller than both the coherence length and the penetration depth, but not so small that the energy levels become discrete. The opposing effects of the pair breaking and of the alignment through the Zeeman term lead to a detailed fit of these calculations to the experimental data. However, more recent results of Tse and MacLaughlin [131, 132], obtained at lower values of the applied magnetic field, seem not to be in complete agreement with Sone's model.

Kobayashi, Takahashi and Sasaki have observed a resonance signal in small copper particles [133]: The line became broader with increasing $\delta / k T$, and the Knight shift did not tend to vanish. As in the case of aluminium (see fig. 3.8b), the low field tail was enhanced, indicating the presence of odd particles. The measurements of Yee and Knight have confirmed these results [134]. In fig. 3.8c the derivative of the absorption signal is shown for copper particles with an average diameter of $10 \mathrm{~nm}$. They also determined the Knight shift of small copper particles in the size range from 2.5 to $11 \mathrm{~nm}$, which were prepared by flash evaporation of successive layers of copper particles isolated with siliconmonoxide; their results are given in fig. 3.9. One of their samples, with an average particle diameter $d \simeq 4 \mathrm{~nm}$, had such a narrow size distribution that the even particle peak was not obscured by the broad background of the odd particles in the low field tail. Therefore, the Knight shift of the even particles in this sample could be studied at higher temperatures as well. A decrease of the Knight shift is found at lower temperatures, the decrease is larger for the smaller particles, and sets in at a higher temperature. Again in disagreement with the predictions of both the orthogonal and symplectic ensemble [105], a residual shift is left for $T \rightarrow 0$. In analogy with the case of superconductors, this residual shift may be attributed to spin reverse scattering preventing pairing of the spins. Yee and Knight have compared their results with the calculations of Abrikosov and Gor'kov [121] and obtained a reasonable agreement. Qualitatively, the results can also be explained with Shiba's theory [127]. The strength of the spin orbit coupling relative to the average level spacing is given by:

$$
\frac{\hbar}{\tau_{\mathrm{so}} \delta}=\frac{\hbar v_{\mathrm{F}}(\Delta g)^{2}}{d \delta}
$$

where $\tau_{\mathrm{so}}$ is the spin lifetime, and $\Delta g$ the $g$-shift as measured with conduction electron spin resonance. 


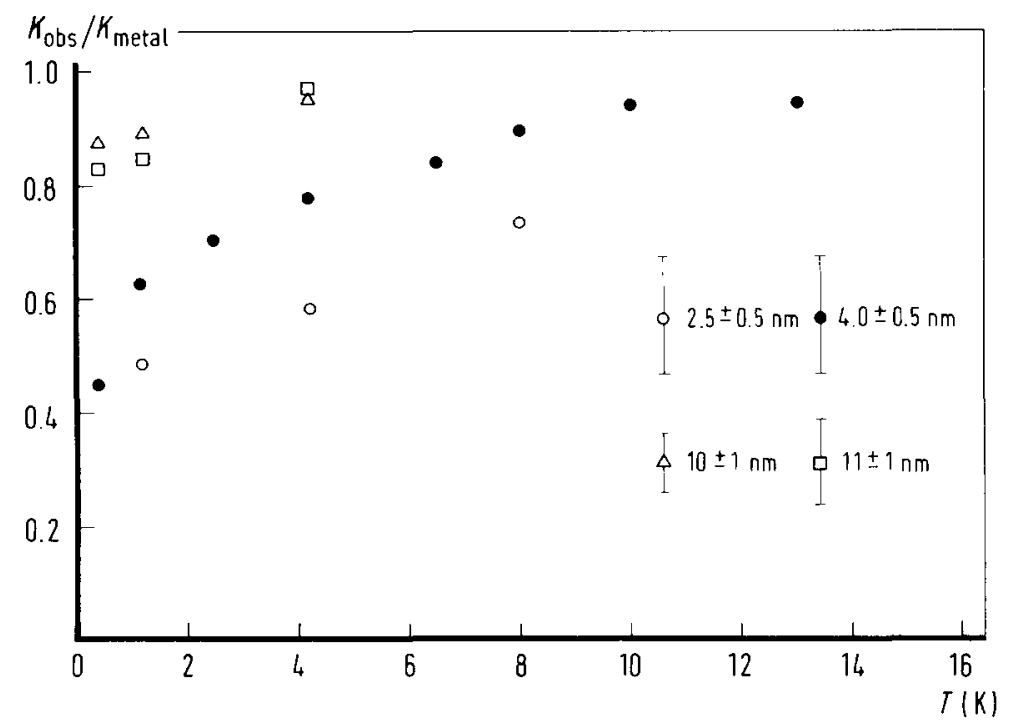

Fig. 3.9. Size and temperature dependence of the Knight shift $K_{\text {obs }}$ observed in flash evaporated copper particles isolated with SiO, normalized to the Knight shift of the bulk metal $K_{\text {metal. }}$ For decreasing particle diameter, the residual Knight shift at zero temperature is found to decrease, indicating a vanishing of the electronic susceptibility of small even particles as a result of quantum size effects. (From Yee and Knight [134].)

This parameter should depend on the particle size as $d^{2}$, but the observed variation seems to be much weaker. According to the arguments of Kawabata [12], spin flip scattering should be blocked by quantum size effects, leading to narrow conduction electron spin resonance lines; this would also favour spin pairing in even particles. The residual Knight shift as observed by Yee and Knight is in agreement with Kubo's prediction of spin pairing in even particles, if one allows for partial inhibition of spin pairing through spin reverse scattering. As was the case with very small tin particles, the depairing becomes weaker with decreasing particle size, in accordance with the blocking of the scattering process as discussed by Kawabata.

Other NMR measurements on fine copper particles were reported by Ido and Hoshino [135], and Hines [136]. Hines prepared the copper particles by flash evaporation as well and the particles had an average diameter of 11 and $15 \mathrm{~nm}$. The width of the resonance line was interpreted by taking into account three still different contributions:

$-\Delta \nu_{\text {bulk }}$, the line width of bulk copper,

$-\Delta \nu_{\mathrm{K}}$, the contribution from the Kubo mechanism (hindered relaxation in the QSE regime), and

$-\Delta \nu_{\mathrm{CH}}$, the contribution due to the Charles and Harrison mechanism [137].

This latter contribution arises from charge density fluctuations near the particle surface resulting from the requirement that the electronic wave functions must vanish at the surface. The nuclei in the range of these fluctuations experience different densities of electrons at their sites, leading to a broadening, with a negligible displacement of the center, of the resonance line. Taking this effect into account, the line width should increase with decreasing particle size. The line widths of the NMR resonances, measured recently on small platinum particles [138], was explained successfully assuming such conduction electron density oscillations. As the Charles and Harrison mechanism was not taken into account by Charvolin et al. $[112,115]$, their interpretation of the experiments on lithium particles is questioned by Hines. More possible relaxation mechanisms are discussed by Knight in his review paper [10], and by Ido and Hoshino [135], who also found an additional field and size dependent contribution to the line width. Very definitely, QSE seems to have been observed by NMR techniques. However, a detailed 
understanding and analysis of all the mechanisms involved in the experiments on the different samples is still lacking.

\subsubsection{Conduction electron spin resonance (CESR)}

CESR is the resonant transition between Zeeman levels of the conduction electrons at $\hbar \omega_{0}=g \mu_{\mathrm{B}} B$. Note that in the size range, where QSE is expected to be important, the particles are so small that the exciting microwave field is not significantly attenuated by the skin effect. The main relaxation mechanism in CESR is due to the spin orbit interaction [101]. For a bulk metal, the spin relaxation time $\tau$ is given by the well known expression:

$$
\tau \simeq \tau_{\mathrm{tr}} /(\Delta g)^{2}
$$

where $\Delta g$ is the electronic $g$-shift, a measure for the strength of the spin orbit coupling, and $\tau_{\mathrm{tr}}$ is the suitably averaged time between two scattering events characteristic for e.g. the electrical conductivity. This relaxation gives a line width of the resonance of $\Delta B \simeq \hbar / g \mu_{\mathrm{B}} \tau$. In small particles, the electron mean free path is limited by the particle dimensions, and then eq. (3.79) leads to

$$
\tau \simeq d / v_{\mathrm{F}}(\Delta g)^{2}
$$

where as usual $d$ is the particle diameter and $v_{\mathrm{F}}$ the Fermi velocity. Eq. (3.80) predicts a broadening of the absorption line, compared with the bulk line width, proportional to $d^{-1}$. When the particle size is reduced even more, the electronic energy levels become discrete and QSE comes into play: The Elliott relaxation mechanism will then be blocked due to the fact that no electronic energy levels are available during relaxation compatible with energy conservation $[12,139]$. In the quantum size limit, the relaxation time should become very long and the corresponding absorption line should be very sharp.

Following Kawabata [12], we summarize the conditions which have to be fulfilled in order to see QSE in CESR:

(1) $\delta \gg \hbar \omega_{0}$ : This condition prohibits energy transfer during relaxation to the kinetic energy of other electrons; the relaxation process is blocked.

(2) $\delta \gg \hbar / \tau$ : This condition is related with the uncertainty principle; the electronic levels of the particles must be sufficiently narrow to prevent overlapping of the levels due to lifetime broadening. Relaxation by spin orbit coupling is then weak enough so as not to mix the energy levels. When both these conditions are fulfilled, a $\delta$-function like absorption line is predicted by Kawabata [12] at the frequency $\Omega=\omega_{0}-\left(\hbar \omega_{0} / \tau \delta\right) f_{i}$, where $f_{i}$ is a constant of the order of 1 , slightly depending on $\delta$ and the details of the energy level distribution. Because of the statistical distribution of $f_{i}$, the resonance line of an assembly of small particles is the envelope of all single electron resonances. For particles of uniform size, one finds $\Delta B \simeq \hbar^{2} \omega_{0} / g \mu_{\mathrm{B}} \tau \delta$. Compared with the bulk metal, the line width is reduced by the factor $\hbar \omega_{0} / \delta$ and is now proportional to $d^{2}$. Expression eq. (3.80) may still be used to calculate $\tau$, but it should be noted that $\tau$ does not have any more the meaning of a lifetime in the strict sense. $\tau$ characterizes the magnitude of the spin orbit matrix elements [12]. In general, it is impossible to measure electron spin resonance in heavy metals due to the large spin orbit interaction. However, when the relaxation mechanism in blocked by the QSE, it should be possible to observe CESR in these metals as well. As the frequency does not depend on the occupation of the electron levels, the shift and the shape of the absorption line are temperature independent; this is in marked contrast to the situation with static (thermodynamic) effects of the QSE. The intensity of the CESR signal is proportional to the 
susceptibility of the conduction electrons and will reveal the predicted quantum size effects in the paramagnetic susceptibility: The vanishing of the susceptibility in particles containing an even number of electrons, as $T \rightarrow 0$, and the $T^{-1}$-law in the susceptibility at low temperatures in particles with an odd number of electrons.

Unfortunately, the experimental results are not yet very coherent, clear, and transparent at this moment. For bulk samples, where no QSE is present, the dependence of the line width of the CESR resonance on the sample dimension appears to be in accordance with eq. (3.80) [140]. At low temperatures, when the bulk mean free path of the electrons is much larger than the sample dimensions, the line width increases with decreasing sample size. This phenomenon is an example of a simple geometrical size effect.

CESR in small particles has been observed in light metals such as lithium [115, 141-146], sodium [147-149], and aluminium [150], as well as in some heavy metals as gold [151, 152], silver [153-156], and platinum [157]. Saiki et al. [145] observed CESR in lithium particles at room temperature. The particles were prepared by evaporation in an inert gas and their average diameter ranged from 300 to $10 \mathrm{~nm}$; the samples were covered with paraffin to prevent oxidation. The results are illustrated in fig. 3.10: For particles larger than $100 \mathrm{~nm}$, the geometrical size effect (a broadening of the absorption line proportional to $d^{-1}$ ) is observed, in agreement with earlier results [141]; for particles smaller than $100 \mathrm{~nm}$, the line width decreases with decreasing particle diameter to a limiting value of $0.2 \mathrm{mT}$. This narrowing of the resonance line, indicating an increase of the transverse relaxation time $T_{2}$, can be attributed to a blocking of the relaxation mechanism as a result of quantum size effects. Borel, Narbel and Monot [146] prepared lithium particles of very small size by simultaneous condensation of $\mathrm{CO}_{2}$ and $\mathrm{Li}$, followed by a heat treatment. The mean sizes of the particles in these samples were estimated to be from 0.7 to $1.8 \mathrm{~nm}$. At $9.3 \mathrm{GHz}$ and at $77 \mathrm{~K}$ the line width for the $0.7 \mathrm{~nm}$ diameter particles was $1.56 \mathrm{mT}$; particles in this small size range showed a line width which is larger than for the much larger particles studied by

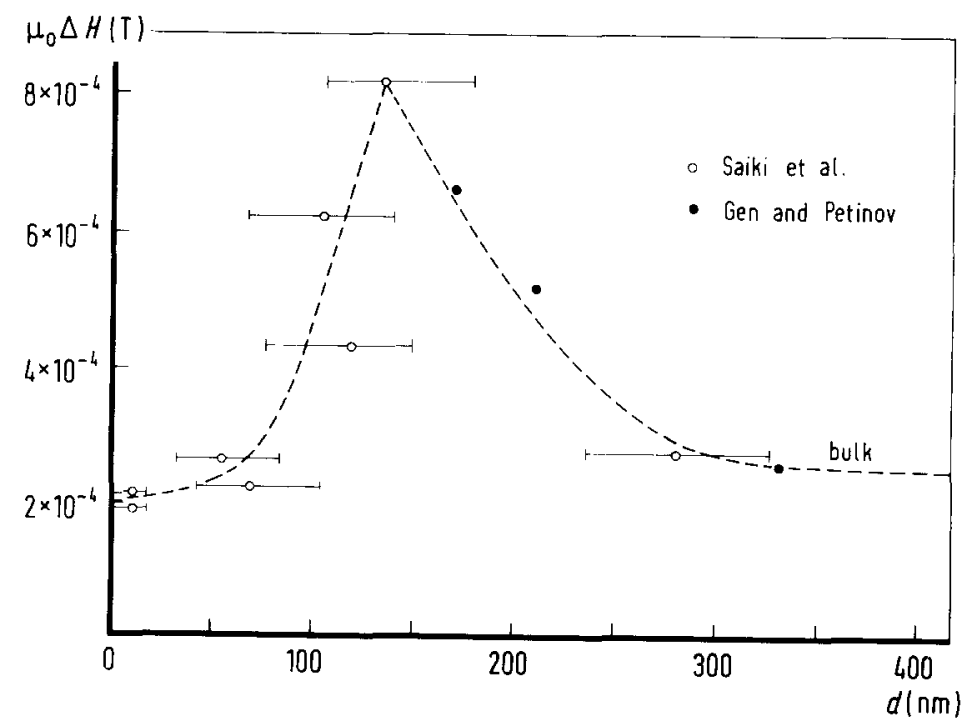

Fig. 3.10. Line width of the conduction electron spin resonance signal for small gas-evaporated particles of lithium as a function of the particle diameter $d$. For particles with a diameter larger than $100 \mathrm{~nm}$, the line width increases with decreasing particle size due to the classical size limitation of the mean free path, in agreement with earlier data of Gen and Petinov. For smaller particles, the line width decreases as a result of the blocking of the relaxation process due to quantum size effects. (From Saiki, Fujita, Shimizu, Sakoh and Wada [145].) 
Saiki et al. At $460 \mathrm{MHz}$, the line widths were between 0.25 and $0.48 \mathrm{mT}$, corresponding to a transverse relaxation time of the order of $2 \times 10^{-8} \mathrm{~s}$. For the sample with a mean particle size of $1.5 \mathrm{~nm}$, the resonance was studied at temperatures below $77 \mathrm{~K}$, and it was observed that the line width did not change much as a function of temperature, whereas the longitudinal relaxation time $T_{1}$, as measured from the saturation curves, increased considerably with decreasing temperature (from $3 \times 10^{-6} \mathrm{~s}$ at $77 \mathrm{~K}$ to $2 \times 10^{-5} \mathrm{~s}$ at $40 \mathrm{~K}$ ). For particles in this size range, the CESR is probably broadened as a result of the distribution of $g$-shifts over the volume and surface regions of the particle, as was discussed by Holland [139], and manifestations of QSE can only be found in the longitudinal relaxation time $T_{1}$.

Gordon carefully studied small sodium particles produced in X-irradiated sodium azide [149], and the temperature and size dependence of the line width was found to follow a relation of the type:

$$
\mu_{0} \Delta H_{\mathrm{pp}}=F(T)+2.60 \times 10^{-12} / d+7.686 \times 10^{-12} / d T
$$

where the first term $F(T)$ indicates a phonon term, connected with bulk properties; the second term is the broadening due to the geometrical size effect, and the third term is attributed to the presence of paramagnetic impurities on the surface of the particles, leading to an extra temperature dependent relaxation mechanism. The line shape of the resonance observed at different temperatures could be analysed with a superposition of Lorentzian curves with line width corresponding to eq. (3.81) and weighted with a suitable distribution of the particle sizes. Earlier data of Smithard [148], from which the bulk term $F(T)$ was subtracted [149], are shown in fig. 3.11. As can be seen, the resonance is not narrowed as predicted by Kawabata's theory. At very low microwave power, a broad and easily saturated resonance was observed [149]; this resonance is attributed to quantum size narrowed resonances, but believed to be broadened inhomogeneously by a distribution of contact hyperfine fields.

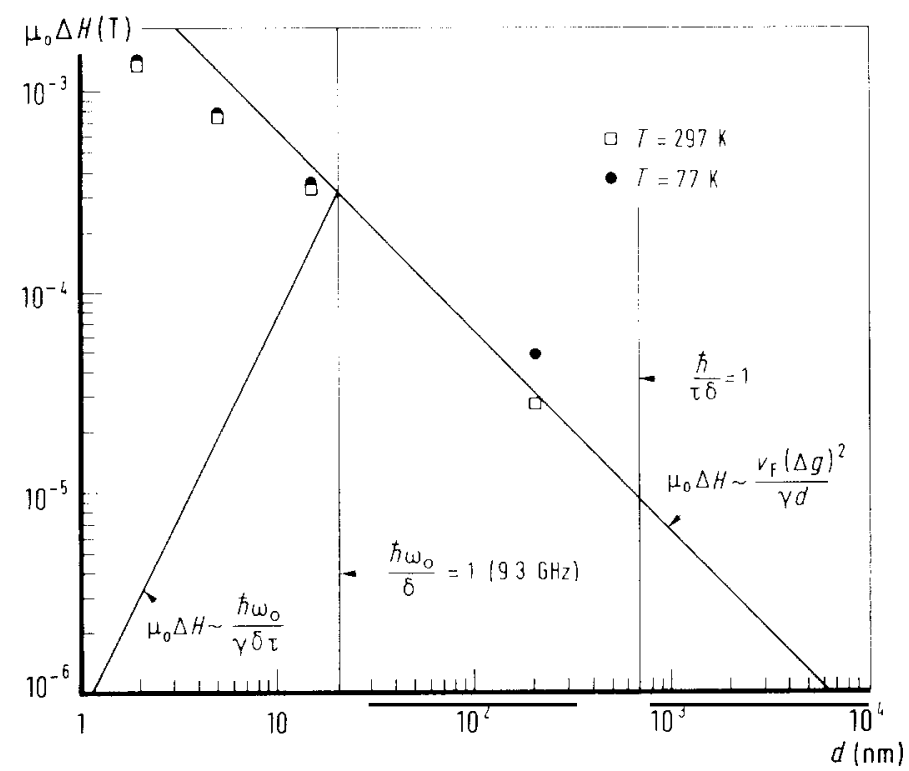

Fig. 3.11. Line width of the conduction electron spin resonance signal for small sodium particles. For large particles, the line width is proportional to the inverse of the classical size limited lifetime of the electrons $d / v_{\mathrm{F}}$. The Kawabata conditions are represented by vertical bars, for $\hbar \omega_{0} \ll \delta$ and $h / \tau \ll \delta$ the relaxation will be reduced with the factor $\hbar \omega_{0} / \delta$ due to quantum size effects. The experimental data of Smithard do not reveal the corresponding narrowing of the resonance. (From Gordon [149].) 


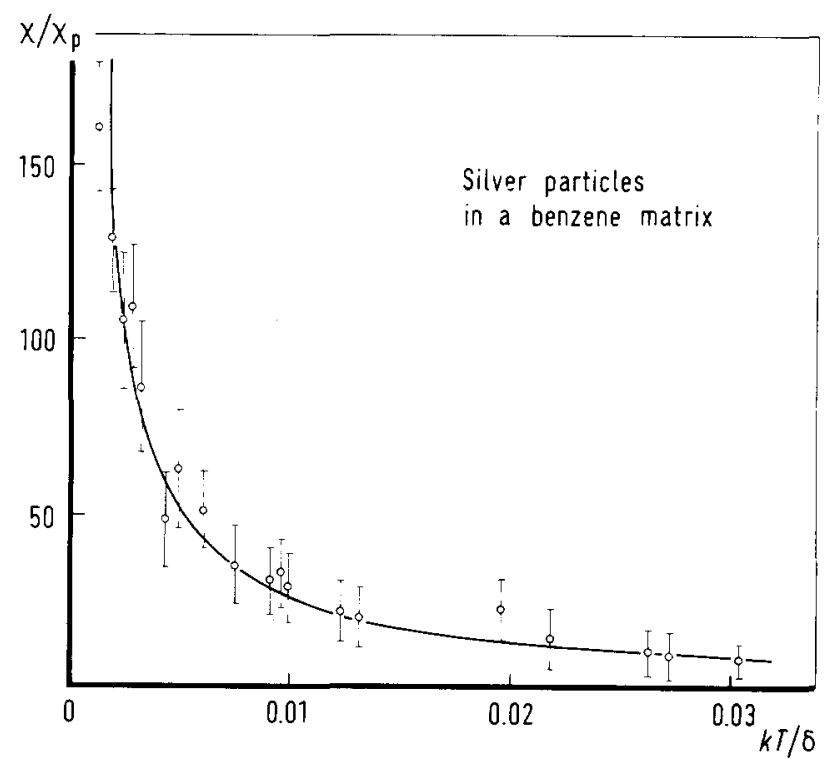

Fig. 3.12. The susceptibility $\chi$ of small silver particles in a benzene matrix, normalized to the Pauli spin paramagnetic susceptibility $\chi_{\mathrm{P}}$, as a function of temperature. $\chi$ was determined from the intensity of the conduction electron spin resonance and has a $T^{-1}$ dependence as expected for odd particles with at least one unpaired electron spin. (From Monot and Millet [156].)

The results obtained for gold particles $[151,152]$ are not very clear either. In the author's laboratory attempts to observe CESR in samples of colloidal gold with diameter from 3.4 to $25 \mathrm{~nm}$ were unsuccessful. It seems that the particle size, below which CESR can be observed, is quite small, but on the other hand the very fact that CESR is observed in small gold particles $[151,152]$ supports the predictions of QSE.

A negative result was also reported by Smithard [29] for silver particles in photosensitive glass. A resonance was observed in silver colloidal particles in $\mathrm{KCl}: \mathrm{Ag}$ crystals, with particles in the size range from 5 to $30 \mathrm{~nm}$. Monot, Narbel and Borel reported spin resonance experiments on silver particles with a diameter in the order of $1 \mathrm{~nm}$ [154]. The proportionality between the shift of the resonance $\left(\hbar \omega_{0} / \tau \delta\right) f_{i}$ and its width $\hbar^{2} \omega_{0} / g \mu_{\mathrm{B}} \tau \delta$, predicted by Kawabata's theory, was verified for more than thirty samples, prepared by reduction of an aqueous solution of $\mathrm{Ag}_{2} \mathrm{O}$ and also by simultaneous condensation of $\mathrm{Ag}$ and $\mathrm{CO}_{2}$. The line shape of the resonances observed in silver particles in various matrix materials was found to be asymmetric. This asymmetry may be related to the existence of a critical size of $2 \mathrm{~nm}$ for CESR in silver particles (corresponding to the conditions given by Kawabata) and the fact that the average size in all samples was in excess of $2 \mathrm{~nm}$, even before annealing [155].

Monot and Millet [156] measured CESR on silver particles prepared by simultaneous condensation of silver and benzene, followed by a heat treatment. The susceptibility of the conduction electrons deduced from the intensity of the CESR signal and determined as a function of temperature showed a $T^{-1}$ dependence at low temperatures, as predicted for odd particles (see fig. 3.12). A similar result was reported for platinum particles with a diameter of $2.2 \mathrm{~nm}$ prepared from a hydrosol [157].

\subsubsection{Far infrared absorption}

The energies corresponding to the average spacing of the electron energy levels under QSE conditions are in the far infrared range of the electromagnetic spectrum. The discrete structure of the 
single electron excitation spectrum was therefore expected to manifest itself directly in the far infrared absorption. Tanner, Sievers and Buhrman [158] studied the far infrared absorption of various samples of lead, copper, aluminium and tin. One of their samples consisting of aluminium particles with an average diameter of $15 \mathrm{~nm}$, had a particularly narrow distribution of sizes; the absorption of this sample is shown in fig. 3.13, and it is tempting to attribute the wiggles in the spectrum to the level structure effects predicted by Gor'kov and Eliashberg [4] as discussed in section 3.4. In a subsequent paper Granqvist, Buhrman, Wyns and Sievers $[159,160]$ convincingly showed that the structure observed is insignificant as far as QSE is concerned. The results of model calculations $[110,159]$, illustrated in fig. 3.14 , show that any practically achievable distribution of particle sizes will tend to smear out the oscillations resulting from the discreteness of the single particle level structure. Therefore, attempts seem to be fortuitous to observe directly the discrete level structure on collections of small particles as they are prepared until now.

A possible way out of this problem would be to do experiments on one or only very few particles. Due to the enormous problems connected with the intensity, it is unrealistic to try to observe far infrared absorption on a single particle, but it has been proposed to do such experiment at optical frequencies [161]. The intensity of the radiation scattered by a small metallic particle falls off quite rapidly with decreasing size (proportional to $d^{6}$, see section 2.2 ), but for particles with a diameter of a few nanometers it is still so strong that they can be observed by eye using an ultramicroscope. This technique was used by Zsigmondy [162] very long ago to study the growth kinetics of colloidal particles. Although the wavelength near the plasma resonance of the small particle is much larger than the particle dimensions, separate spots have been observed when the colloidal system is sufficiently dilute. If an optical laser with a band width smaller than the expected mean level spacing is used to excite the collective surface plasma mode, under favourable conditions the scattered radiation will reflect the level structure of the single electron excitations, and these effects can be studied with usual Raman

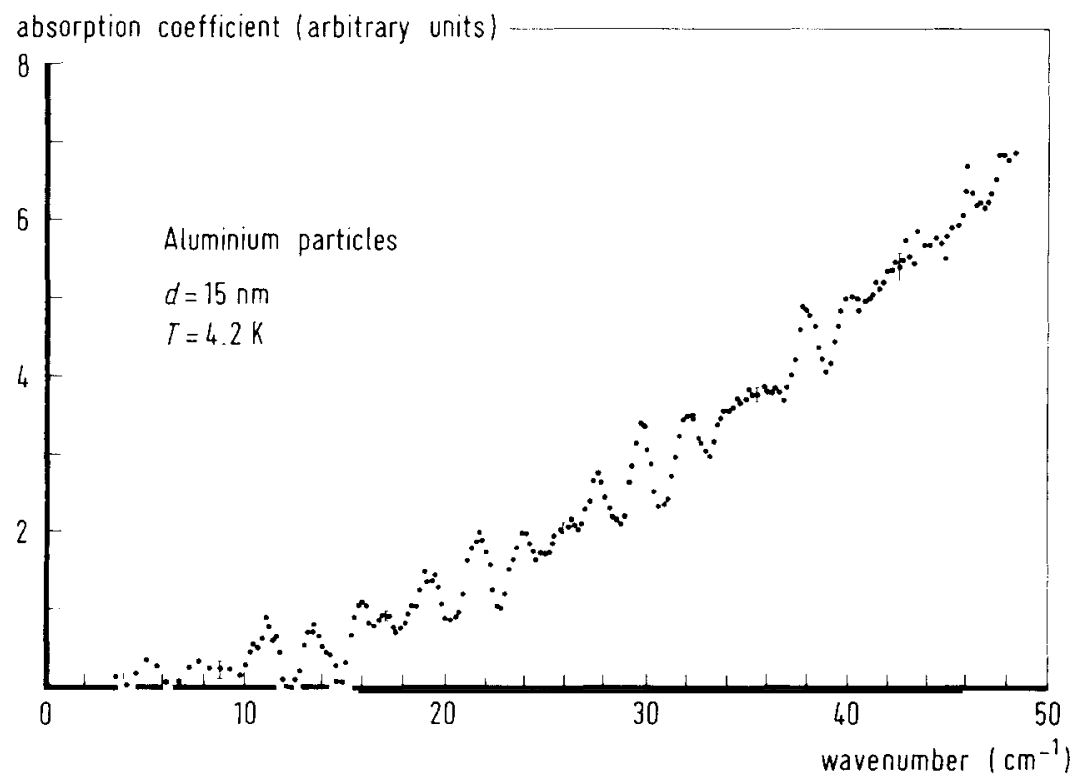

Fig. 3.13. Far infrared absorption spectrum of small gas-evaporated aluminium particles with an average diameter of $15 \mathrm{~nm}$. The oscillations of the absorption coefficient as a function of the wavenumber are well in excess of the instrumental resolution; but most probably, they are not due to the discreteness of the electronic level spectrum. (From Tanner, Sievers and Buhrman [158].) 


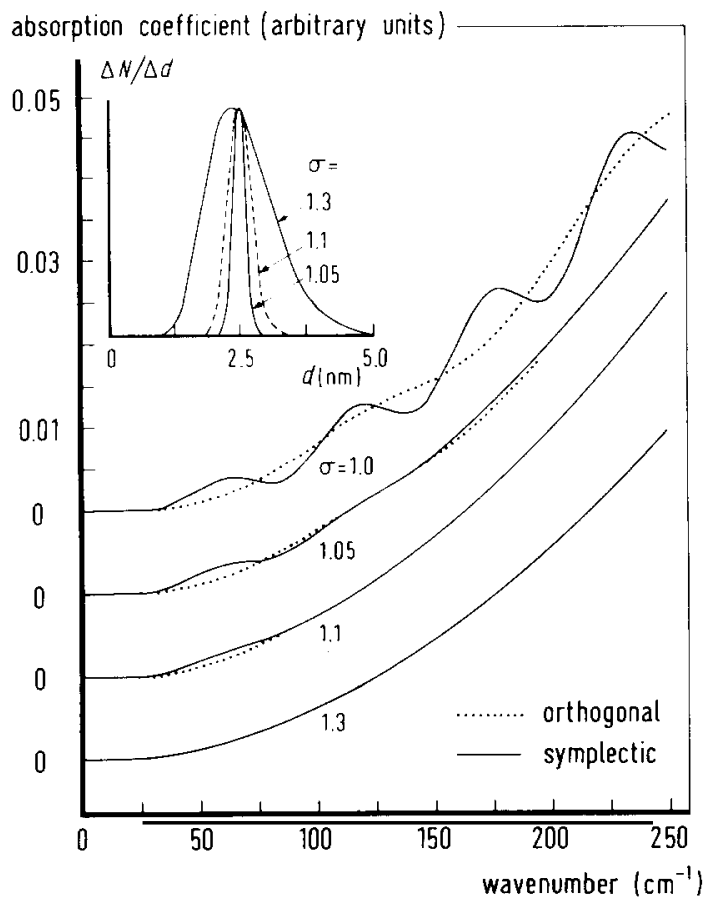

Fig. 3.14. Model calculations of the absorption coefficient of small metallic particles in the far infrared. The absorption was calculated using the Gor'kov-Eliashberg theory and an additional averaging over different particle sizes was done using a log-normal particle size distribution, with different values for the width $\sigma$ but with a constant average particle diameter of $2.5 \mathrm{~nm}$ (see insert). For the symplectic ensemble large oscillations of the absorption coefficient are found, but these soon disappear when the particles are not of uniform size. (From Granqvist, Buhrman, Wyns and Sievers [159].)

spectroscopic techniques. A number of serious problems must be overcome: The effective temperature of the conduction electrons must be so low that only very few levels are partly occupied to prevent smearing out of the discrete structure of the single electron excitation spectrum; moreover, coincidence measurements will be necessary to determine the energy level correlation function, as the time available for measurements is probably very short. The proposed experiment will be rather difficult to perform, but a more direct observation of the discrete structure of the energy levels resulting from QSE would be of invaluable importance.

So, unfortunately, far infrared absorption measurements do not give direct information on the single electron excitation spectrum $[159,160]$. Nevertheless, the overall absorption was found to vary with the frequency $\nu$ approximately as $\nu^{2}$ (see fig. 3.13), in agreement with both the Gor'kov and Eliashberg [4], and the classical Drude theory [159]. However, the absorption coefficient is orders of magnitude larger than calculated from all these theoretical models. Moreover, the Gor'kov-Eliashberg theory leads to a $d^{-1}$ dependence of the absorption coefficient, whereas the far infrared absorption was found to increase with increasing particle size. Lushnikov, Maksimenko and Simonov have reconsidered the original work of Gor'kov and Eliashberg, and they have calculated the far infrared absorption taking into account the Coulomb interaction between the conduction electrons as well [163]; this calculation gives correctly the experimentally observed size dependence of the absorption coefficient, but still does not reproduce the high value found in the experiments. Several extra absorption mechanisms have been proposed to explain the experimental results, such as eddy currents, the effects of amorphous oxide layers, or direct excitation of surface phonons [164-168]. Stroud and Pan [164] have shown that when eddy currents are 
taken into account the absorption coefficient, calculated using an effective medium approach, is a few orders of magnitude bigger than the Maxwell Garnett result. The effective medium value is found in the usual way, i.e. fluctuations due to the fields scattered from the inclusions are assumed to average out to zero, and the forward scattered field is now calculated using eq. (2.11a); the Mie expression is evaluated to higher order, whereas only the lowest order contribution was contained in the original Maxwell Garnett theory.

A very detailed discussion of the far infrared absorption of very small metallic particles was given by Granquist [168]. Predictions of the original Maxwell Garnett theory and of Bruggeman's effective medium theory were compared, and the influence was discussed of effective depolarization factors which can take into account local field effects. It was shown that the effect of eddy currents can be included with an effective magnetic permeability of the medium arising from the induced magnetic dipole moments of the small particles; this leads to an extra term in the absorption which becomes important for particles larger than about $5 \mathrm{~nm}$. The size dependence is different for very small and for larger particles: Proportional to $d^{-1}$ when eddy currents can be neglected, and proportional to $d^{3}$ for larger particles.

\subsubsection{Static electric susceptibility}

In their original paper [4], Gor'kov and Eliashberg predicted a strong enhancement of the electrical polarizability of a system of small metallic particles if the applied electric field $E$ is not too big $(e E d \ll \delta)$. They foresaw technical applications in the form of artificial dielectrics with large and controllable dielectric constants. However, in two different sets of experiments on small metallic particles dispersed in a glass matrix, no such anomalous effects were observed $[169,170]$. The reason is discussed in section 3.4: Not the polarizability $\alpha$, but the susceptibility $\chi_{\mathrm{p}}$ has been calculated, as the effect of the depolarizing field of the induced electric dipole moment was not taken into account. The expression for the polarizability of a small particle is given in eq. (3.65). For a very big particle, with perfect screening of the external field as in the bulk metal, the susceptibility tends to infinity, and one gets the familiar result of classical electrostatics: $\alpha=\frac{1}{2} \pi d^{3}$. When the quantum size effect is taken into

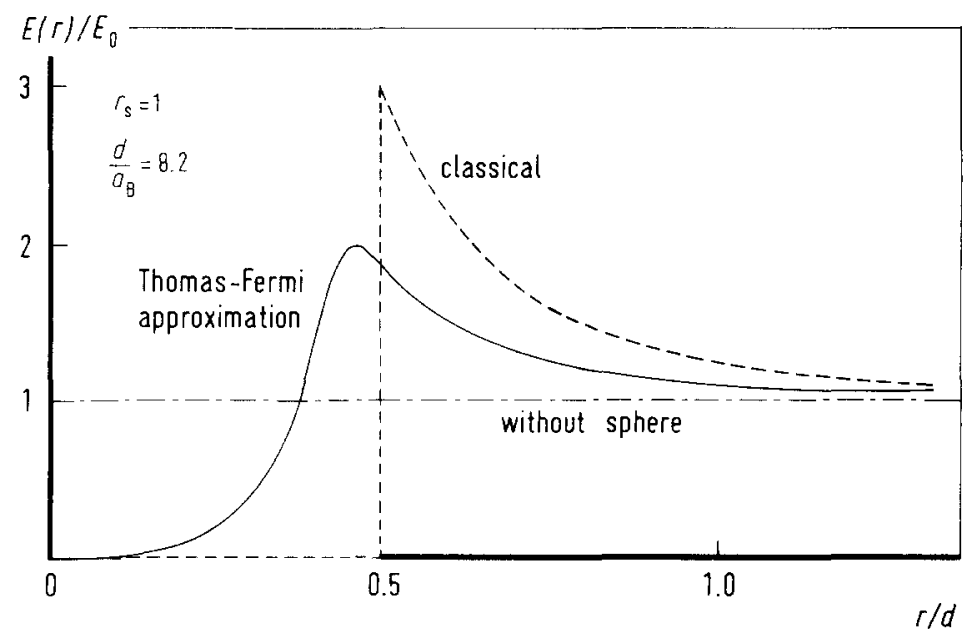

Fig. 3.15. The results of a selfconsistent calculation of the electric field $E(r)$ inside a metallic particle when the effect of the Thomas-Fermi screening length is taken into account (full curve), and the classical result where the interior of the particle is perfectly shielded from the applied electric field $E_{0}$. The electronic density parameter $r_{\mathrm{s}}$ is related to the mean electronic density $n$ by $r_{\mathrm{s}}=\left(3 n / 4 \pi a_{\mathrm{B}}^{3}\right)^{1 / 3}$, and the Bohr radius $a_{\mathrm{B}}$ is $0.053 \mathrm{~nm}$. (From Rice, Schneider and Strässler [106].) 
account, the polarizability of a small particle is slightly smaller than that of the corresponding metallic particle [107]. As $\chi_{\mathrm{p}} \gg 1$ the difference is very small: For a gold particle of diameter $10 \mathrm{~nm}$ with $\delta \simeq 0.1 \mathrm{meV}, \alpha$ is roughly $1 \%$ smaller than the classical value, whereas the erroneously supposed enhancement of the polarizability would be a factor of the order of 300 .

If the Thomas-Fermi screening length of the electron gas becomes comparable to the dimensions of the particle, the assumption of a spatially uniform electric field inside the particle is no longer true, as can be seen in fig. 3.15. The polarization of the particle is then determined by this varying internal field, and has to be calculated selfconsistently. This problem has been treated in detail by Rice, Schneider and Strässler [106], and Cini and Ascarelli [42].

\subsubsection{Static magnetization}

As discussed in section 3.5.1, NMR experiments are very well suited to study the susceptibility of the conduction electrons and to look for QSE. However, the contribution from the unpaired electron in "odd" metallic particles has not been detected unambiguously by NMR techniques due to the fact that the size distribution tends to smear out the volume susceptibility associated with the polarization of this single electron. Under favourable conditions, the presence of odd particles is noticeable only in the deformation of the resonance line of particles with an even number of electrons. Obviously, it is of great interest to do an experiment where the particles with an odd number of electrons manifest themselves in a more direct way, and where the non-uniformity of size does not to a large extent mask the expected effects. The simplest experiment to study the "odd" case is the measurement of the static magnetization. If $\delta / k T \gg 1$ and $\mu_{0} \mu_{\mathrm{B}} H<\delta$, the contribution of the even particles to the magnetic moment vanishes, whereas the odd particles behave as if each had a single free electron spin.

Measurements of the dc magnetic susceptibility can be used to study the appearance of the Meissner effect in small superconducting particles as well; this will be discussed in some detail in section 4. Akoh and Tasaki [171] measured the susceptibility of small vanadium particles $(d>9 \mathrm{~nm})$ and observed a Curie-Weiss type contribution to the paramagnetic susceptibility which increased with decreasing particle size, proportional to the fraction of surface atoms and which they therefore attributed to magnetic moments arising from localization of $3 d$ orbitals at the particle surface. Magnetization measurements have also proved to be a powerful tool for the study of the superparamagnetic behaviour of small, single-domain, ferromagnetic particles.

Only in very few investigations the dc magnetization has been used to look for quantum size effects in the susceptibility of the conduction electrons [172-174]. Most workers favour the electron spin resonance technique which is much more sensitive than conventional susceptometers. However, in magnetization measurements, the magnetic field can be varied at wish, and therefore at temperatures of about $1 \mathrm{~K}$ it should be possible to observe saturation effects of the magnetization of the conduction electrons in magnetic fields $B<10 \mathrm{~T}$, easily accessible with standard superconducting magnets. Marzke and coworkers [172] have measured the susceptibility of $2.2 \mathrm{~nm}$ diameter platinum particles. The samples were prepared from platinum hydrosols and were stabilized using gelatin. Below $20 \mathrm{~K}$ the susceptibility obeyed a $T^{-1}$-law corresponding to approximately one spin on every two particles. However, the magnitude of this contribution seems not to be compatible with related CESR measurements [157]. Meier and Wyder measured the magnetization of indium particles embedded in paraffin $[173,174]$. The indium metal was evaporated into a flow of helium gas and the aerosol so obtained was passed through molten paraffin. The paraffin acted as a filter and an appreciable amount of the particles was trapped, well separated from each other. The magnetic moment of the samples was measured by means of a vibrating sample magnetometer in the temperature range from 4.2 to $1.5 \mathrm{~K}$, and in magnetic 
fields up to $5 \mathrm{~T}$. In this temperature range, the magnetization was found to be only weakly temperature dependent (as compared to a $T^{-1}$-law). After subtraction from the data of a negative contribution, linearly dependent on the magnetic field $B$, and believed to be due to the diamagnetism of the paraffin, a paramagnetic contribution to the magnetization was found which was a strongly non-linear function of $B$ and which saturated at temperatures of $\simeq 4 \mathrm{~K}$ at fields of approximately $2 \mathrm{~T}$ [174]. Attempts to explain the observed magnetization within Kubo's model of free spins were doomed to be unsuccessful because the data did not scale with the parameter $\mu_{B} B / k T$. It was suggested that the relatively low saturation field of the paramagnetic part of the signal could be due to large orbital momenta associated with the nearly spherical boundary conditions, imposed on the states of the electrons. This high symmetry introduces a very strong degeneracy of the electronic energy levels. In a more elaborate discussion, van Gelder [175] was able to modify this simple model to include the observed temperature dependence. The degenerate levels of the Zeeman multiplets are now assumed to be split up in a random way as a result of random perturbations from perfect sphericity. When the remaining linear dependence on $B$ for high fields was subtracted from the calculated data (as was done in the experiment of Meier and Wyder) curves as shown in fig. 3.16 were obtained. Fig. 3.16b shows that van Gelder's theory can give a good description of the experimental results. Separate measurements of the magnetization at $77 \mathrm{~K}$ did not show any non-linearity in the magnetization curves, in agreement with the theoretical models.

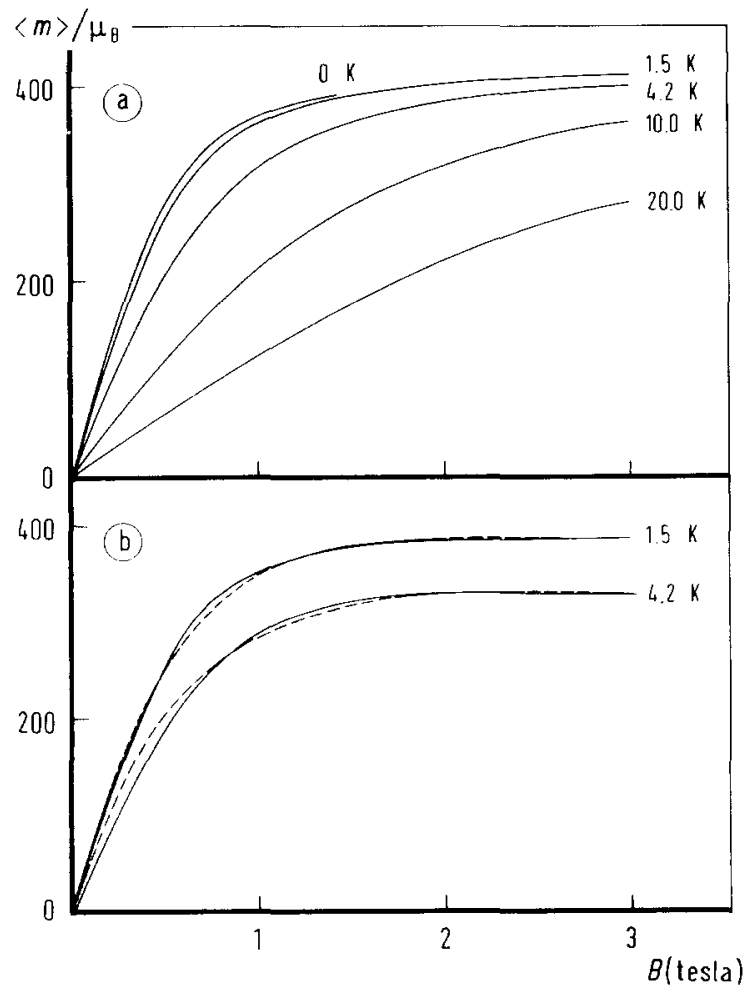

Fig. 3.16. Calculated magnetic moment of small spherical particles as a function of temperature. For the calculation it was assumed that the appropriate eigenfunctions of the electrons are the spherical harmonics with large orbital quantum numbers; furthermore, a random contribution to the Hamiltonian was considered to take into account the effects of deviations from the ideal spherical symmetry due to e.g. roughness of the particle surface. The experimental results of Meier and Wyder are indicated in (b) by the dashed line and they seem to be in good agreement with the calculated curves, which were obtained after subtraction of the linear part of the magnetization for fields above 2 tesla from the curves given in (a). (From Van Gelder [175].) 
Nevertheless, it was the general feeling that these experimental data provided only a very restricted basis for conclusions regarding the presence of quantum size effects. More detailed information about the transition to bulk-like behaviour, about the correlation of the observed effects with particle size, and the temperature dependence of the effect over a much wider range was badly needed. For measurements of this type a very sensitive and versatile magnetometer was constructed [176], and recently the magnetization of indium particles was measured over an extended range of temperatures $(2.5$ to $300 \mathrm{~K})$ and with improved sensitivity $[176,177]$. The temperature dependence of the magnetization of samples containing indium particles embedded in paraffin was found to be a function of $\mu_{\mathrm{B}} B / k T$, in disagreement with van Gelder's theory and the earlier interpretation of the experimental data. Typical magnetization data are presented in fig. 3.17, the full curve represents the effective moment calculated from the occupation probabilities of a spin $S=1$ Zeeman triplet, the data correspond to five series of measurements as a function of $T$ at different values of $B$. After subtraction of the temperature dependent contribution $m_{1}(B / T)$, a linear function of $B$ was left, and this contribution was attributed to the (field independent) diamagnetic susceptibility of the sample. In only a few of the samples, the remaining contribution was a strongly non-linear function of $B$. But after subtraction of the linear function observed in the high field limit, a temperature independent contribution was obtained, which saturated at fields well below $1 \mathrm{~T}$ and which was attributed to a contamination of the sample with very small pieces of iron. In the earlier experiments by Meier and Wyder [174] such contaminations have probably been mistaken for a small particle effect, as the different contributions to the magnetization could not be discriminated in the very limited temperature range available.

As an order of magnitude estimate, one can compare the volume susceptibility associated with one unpaired spin in a particle of volume $V_{0}\left(\chi=\mu_{0} \mu_{\mathrm{B}}^{2} / k T V_{0}\right)$ with the Pauli spin paramagnetic susceptibility $\left(\chi_{\mathrm{P}}=\mu_{0} \mu_{\mathrm{B}}^{2} \rho\left(\varepsilon_{\mathrm{F}}\right)\right)$. As the quantum size parameter $\delta$ scales with $N^{-1}$, and hence with $V_{0}^{-1}$, one obtains for indium particles in this regime: $\chi \simeq 6.8 \times 10^{-6} \delta / k T . \chi$ has become comparable with the bulk Pauli susceptibility when the latter starts to deviate from the normal bulk value as a result of QSE $[3,105]$. The paramagnetic susceptibility will dominate when $V_{0} T<4.2 \times 10^{-25} \mathrm{~m}^{3} \mathrm{~K}$. Therefore, QSE

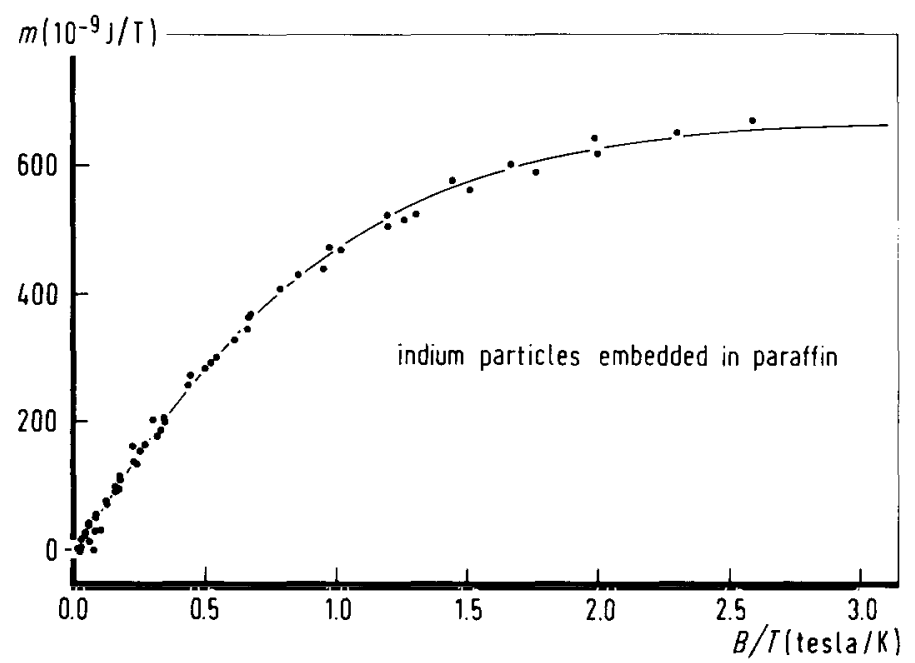

Fig. 3.17. The magnetic moment of a collection of small gas-evaporated particles of indium embedded in paraffin as a function of $B / T$. The data represent the results of five series of measurements at different values of the applied magnetic field; for each value of $B$, an asymptotic value of $m$ for $T \rightarrow \infty$ was determined and subtracted from the experimental data. The magnetization closely follows a curve which represents a common Langevin function characterized by a spin quantum number $S=1$. (From Perenboom, Wyder and Meier [177].) 
will occur in indium particles smaller than $6 \mathrm{~nm}$ at temperatures approaching $1 \mathrm{~K}$. The measured paramagnetic contribution $m_{1}(B / T)$ was a few orders of magnitude bigger than expected for a single spin per particle, and was attributed to oxygen trapped in the paraffin matrix. Therefore, it had to be concluded that no quantum size effects have been observed in small indium particles [177].

\section{Superconductivity in small particles}

The problem of superconductivity in small particles attracted a great deal of interest over many years [178]. We will not discuss the very important surface effects which are more accessible in small particles than in bulk material (e.g. electron-electron interaction due to surface phonons [179]), but again in the spirit of this paper we will concentrate on questions, where the smallness of the superconducting particle is essential in order to lead to new effects such as size effects, QSE, magnetic properties, thermal fluctuations, and on the question of a lower size limit for superconductivity itself.

\subsection{Characteristic lengths of superconductors}

Three characteristic lengths, relevant in superconductors, can be compared with the dimensions of the particles [180]: The penetration depth $\lambda$, characterizing the spatial penetration of a magnetic field into a superconductor; the coherence length $\xi_{\Delta}$, characterizing the spatial "variation of superconductivity" as measured by the superconducting order parameter $\Delta(\boldsymbol{r})$; and the coherence length $\xi_{\boldsymbol{A}}$ of Pippard, characterizing the non-locality of the electrodynamic response function with respect to the vector potential $\boldsymbol{A}$. In addition, the problems of a discontinuous energy level structure (QSE) have to be considered in small superconductors as well. These characteristic lengths can be calculated with the BCS microscopic theory of superconductivity [181] and the more modern version thereof [182]. In an impure superconductor, the Pippard coherence length is approximately given by

$$
1 / \xi_{A}=1 / \xi_{0}+1 / l
$$

where $l$ is the electronic mean free path and $\xi_{0}=0.18 \hbar v_{\mathrm{F}} / k T_{\mathrm{c}}\left(v_{\mathrm{F}}\right.$ : Fermi velocity, $T_{\mathrm{c}}$ : critical temperature). Obviously, $\xi_{\boldsymbol{A}} \simeq l$ if $l \ll \xi_{0}$. In the Ginzburg-Landau approximation [183], i.e. for temperatures $T$ close to $T_{c}, \xi_{\Delta}(T)$ is given by

$$
\xi_{\Delta}(T) \simeq \xi_{0}\left(\frac{T_{\mathrm{c}}}{T_{\mathrm{c}}-T}\right)^{1 / 2} \quad\left(l \gg \xi_{0}\right)
$$

or

$$
\xi_{\Delta}(T) \simeq\left(\xi_{0} l \frac{T_{\mathrm{c}}}{T_{\mathrm{c}}-T}\right)^{1 / 2} \quad\left(l \ll \xi_{0}\right)
$$

For the penetration of a magnetic field in a small superconductor, an effective penetration depth $\lambda_{\text {eff }}$ can be defined. For the dirty case, the BCS theory [181] leads at $T=0$ to

$$
\lambda_{\mathrm{eff}}=\lambda_{\mathbf{L}}\left(\xi_{0} / \xi_{\mathbf{A}}\right)^{1 / 2} \simeq \lambda_{\mathbf{L}}\left(\xi_{0} / l\right)^{1 / 2}
$$


where $\lambda_{\mathrm{L}}=\left(m / \mu_{0} n e^{2}\right)^{1 / 2}$ is the London penetration depth defined in the conventional way in terms of the properties of the pure normal metal [180] ( $m$ and $e$ denote the mass and the charge of the conduction electrons, and $n$ their density per unit volume). Apart from a numerical factor which is 0.86 at $T_{\mathrm{c}}$, a corresponding relation is valid for $T>0$. If the particle is clean so that $l \gg \xi_{0} \gg \lambda>d$, where $d$ is the particle diameter, the non-locality of the electrodynamics must be taken into account and one gets

$$
\lambda_{\text {eff }}=\lambda_{\mathrm{L}}\left(\xi_{0} / d\right)^{1 / 2}
$$

For most superconductors, these intrinsic lengths $\lambda_{\mathrm{L}}$ and $\xi_{0}$ are of the order of a few hundred $\mathrm{nm}$.

\subsection{Magnetization of small superconducting particles}

Surprisingly enough, there exist very few investigations on the magnetization of small superconducting particles, although magnetization measurements should give a considerable amount of information on the more thermodynamic properties of small superconductors. Most of the magnetic measurements on small superconductors are simple determinations of $T_{\mathrm{c}}$ by monitoring the susceptibility in order to study the size dependence of superconductivity [184].

If the size of the superconducting particle is small compared with the penetration depth $\lambda_{\text {eff }}$, according to the Ginzburg-Landau theory [180] a second order phase transition towards the normal state is to be expected in a magnetic field [185]; therefore all superheating or supercooling effects will disappear. From a pure phenomenological point of view, the magnetic properties of a small superconductor can be described by a London equation [186]:

$$
\nabla^{2} B=B / \lambda^{2}
$$

Here, due to the non-locality of the electrodynamics and the field dependence of the penetration depth $\lambda$, an appropriate $\lambda_{\text {eff }}$ has to be used as sketched in eqs. (4.4) and (4.5). For a sphere of diameter $d$ in a uniform field $B_{0}$, the solution of (4.6) (expressed in polar coordinates and with the direction of the field as the axis of reference [186]) is given by:

$$
\begin{array}{ll}
r \leq \frac{d}{2}: & B_{r}=\frac{3}{2} B_{0} \frac{\lambda d}{r^{2}} \frac{\sinh (r / \lambda)}{\sinh (d / 2 \lambda)}\left[\operatorname{coth}\left(\frac{r}{\lambda}\right)-\frac{\lambda}{r}\right] \cos \theta \\
B_{\theta} & =\frac{3}{4} B_{0} \frac{\lambda d}{r^{2}} \frac{\sinh (r / \lambda)}{\sinh (d / 2 \lambda)}\left[\operatorname{coth}\left(\frac{r}{\lambda}\right)-\frac{\lambda}{r}\left(1+\frac{r^{2}}{\lambda^{2}}\right)\right] \sin \theta \\
B_{\phi} & =0 \\
r>\frac{d}{2}: & B_{r}=\left[B_{0}+2 \mu_{0} m / 4 \pi r^{3}\right] \cos \theta \\
B_{\theta} & =\left[-B_{0}+\mu_{0} m / 4 \pi r^{3}\right] \sin \theta \\
B_{\phi} & =0
\end{array}
$$

where:

$$
\mu_{0} m=-\frac{\pi}{4} B_{0} d^{3}\left(1-\frac{6 \lambda}{d} \operatorname{coth}\left(\frac{d}{2 \lambda}\right)+\frac{12 \lambda^{2}}{d^{2}}\right) .
$$


The leading factor $-(\pi / 4) B_{0} d^{3}$ is equal to the magnetic moment induced in a perfectly diamagnetic sphere. Eq. (4.7b) shows that the field outside the sphere is the original one together with that of the induced magnetic dipole moment $m$. For the magnetic moment per unit volume $M$, one has

$$
\frac{M}{M_{0}}=1-\frac{6 \lambda}{d} \operatorname{coth}\left(\frac{d}{2 \lambda}\right)+\frac{12 \lambda^{2}}{d^{2}}
$$

where

$$
\mu_{0} M_{0}=-\frac{3}{2} B_{0}
$$

For small particles, eq. (4.9) reduces to

$$
\frac{M}{M_{0}} \simeq \frac{1}{60} \frac{d^{2}}{\lambda^{2}} \quad(d \ll \lambda)
$$

When we take into account the size dependence of the London penetration depth, given in eq. (4.5), we can rewrite this relation as:

$$
\frac{M}{M_{0}}=\frac{1}{60} \frac{d^{3}}{\lambda_{\mathrm{L}}^{2} \xi_{0}}
$$

On the basis of this simple London theory (eq. (4.6)) it is possible to make an estimate of the critical magnetic field of a small superconducting particle. If the magnetostatic energy required to expel the field from the interior of the sphere is equal to the superconducting condensation energy, the particle exhibits a first order phase transition into the normal state. From the condition that these energies are equal, the critical field of a small particle $(d \ll \lambda)$ is given from the London theory as

$$
H_{\mathrm{c}}=\sqrt{60} H_{\mathrm{B}}\left(\lambda_{\mathrm{L}} / d\right)
$$

where $H_{\mathrm{B}}$ is the thermodynamic critical field of the bulk material.

It is well known that this London approach is inadequate, as it does not take into account non-linearities in the response of the superconductor to the static magnetic field. These can most appropriately be included with the help of the celebrated Ginzburg-Landau equations (for an excellent analysis of these equations see e.g. ref. [180]).

If, according to Ginzburg-Landau, the order parameter $\Delta$ characterizing the superconducting state varies spatially, the minimization of the free energy density of the superconductor leads to the following equation, using the conventional notation [180]:

$$
\frac{1}{4 m^{*}}(-\mathrm{i} \hbar \nabla-2 e A)^{2} \Delta(r)+a \Delta(r)+b|\Delta(r)|^{2} \Delta(r)=0 .
$$

The phenomenological constants $1 / 4 m^{*}, a$ and $b$ can either be related to quantities accessible by experiments (like the thermodynamic bulk critical field $H_{\mathrm{B}}$, the London penetration depth $\lambda_{\mathrm{L}}$, and the coherence length $\xi_{\Delta}$ ), or can be calculated directly from the microscopic theory of superconductivity. The gauge of the vector potential $\boldsymbol{A}(\boldsymbol{r})$ has to be chosen appropriately, and the boundary condition 


$$
n \cdot(-\mathrm{i} h \boldsymbol{\nabla} \Delta-2 e \boldsymbol{A} \Delta)=0
$$

has to be fulfilled, where $\boldsymbol{n}$ is the vector normal to the surface of the body.

In fields somewhat below the critical field $H_{\mathrm{c}}$ of the superconducting particle, the superconducting phase has to have a small value of the order parameter, i.e. $\Delta \rightarrow 0$ if $H \rightarrow H_{c}$ (second order phase transition). Therefore, eq. (4.14) can be linearized with respect to $\Delta$, and one obtains

$$
\frac{1}{4 m^{*}}(-\mathrm{i} \hbar \nabla-2 e A)^{2} \Delta=|a| \Delta
$$

where $\boldsymbol{A}$ denotes the vector potential of the uniform external field $\boldsymbol{B}$ for $\Delta=0$, when the particle is in the normal state and the external field is undisturbed. Eq. (4.16) has the famous and well known form of the Schrödinger equation for a particle of mass $2 m^{*}$ and charge $2 e$ in a magnetic field, with $|a|$ as the energy eigenvalue. Therefore, the problem amounts to the determination of the lowest eigenvalue of this Schrödinger equation. In the limit of a small particle $\left(d \ll \lambda_{\mathrm{L}}\right)$, this can be done by perturbation theory, using the magnetic field as a perturbation and $\Delta(r)=$ constant as the unperturbed wave function. For the uniform field $\boldsymbol{B}$, the vector potential can be taken as $\boldsymbol{A}(\boldsymbol{r})=\frac{1}{2} \boldsymbol{B} \times \boldsymbol{r}$; this gauge, together with the spatially constant order parameter, satisfies the boundary conditions at the surface of the particle, i.e. $\boldsymbol{n} \cdot \boldsymbol{A}=0$. In first order perturbation theory, the lowest eigenvalue is given by

$$
E_{0}=\frac{1}{V} \int \frac{e^{2}}{4 m^{*}} B^{2} r^{2} \sin ^{2} \theta \mathrm{d} V=\frac{e^{2}}{40 m^{*}} B^{2} d^{2}
$$

where $\theta=\measuredangle(B, r)$. When the magnetic field is decreased, superconductivity will reappear in the small particle at a field strength given by the condition $E_{0}=|a|$. Rewriting the phenomenological constants of the Ginzburg-Landau equation in terms of the London penetration depth, one gets for the critical field of a small superconducting sphere in the Ginzburg-Landau approximation

$$
H_{\mathrm{c}}=\sqrt{80} H_{\mathrm{B}}\left(\lambda_{\mathrm{L}} / d\right)
$$

As expected, this result is slightly higher than the result of the simple London theory given in eq. (4.13).

It has to be realized that even for very small superconducting particles without any Meissner effect there exists a theoretical upper limit for the critical field $H_{c}$; this is the so-called paramagnetic limit of Clogston [187]. The condensation energy of a superconductor $\Delta F$ has to be larger than the difference in the paramagnetic energy between the normal and the superconducting state: $\Delta F>\frac{1}{2} \mu_{0}\left(\chi_{\mathrm{N}}-\chi_{\mathrm{s}}\right) H^{2}$ $\left(\chi_{\mathrm{N}, \mathrm{s}}:\right.$ magnetic susceptibility in the normal and superconducting state). Neglecting orbital contributions to the susceptibility, and assuming zero susceptibility in the superconducting state, one gets from the BCS theory $\mu_{0} H_{\mathrm{c}}^{\max }(0)=1.84 T_{\mathrm{c}} \mathrm{T}$.

\subsection{Microscopic theory of small superconductors}

\subsubsection{Basic equations}

The more modern treatments of the general microscopic theory of superconductivity are based on the Gor'kov equations [182]. In this approach a superconductor in an external magnetic field is described by the following set of coupled equations: 


$$
\begin{aligned}
& \left(\mathrm{i} \hbar \omega_{n}-\hat{H}\right) G\left(r, r^{\prime}, \omega_{n}\right)+\Delta^{*}(r) F\left(r, r^{\prime}, \omega_{n}\right)=\hbar \delta\left(r-r^{\prime}\right) \\
& \left(\mathrm{i} \hbar \omega_{n}+\hat{H}^{*}\right) F\left(r, r^{\prime}, \omega_{n}\right)+\Delta(r) G\left(r, r^{\prime}, \omega_{n}\right)=0 .
\end{aligned}
$$

In these equations, $G$ and $F$ are the usual temperature dependent Green's functions; for finite temperatures, the Matsubara frequencies $\hbar \omega_{n}=(2 n+1) \pi k T$ guarantee the proper Fermi statistics. $\hat{H}$ is the full electron Hamiltonian measured from the chemical potential, and includes the interaction of the electrons with boundaries, with impurities and with the magnetic field. $\hat{H}$ differs from $\hat{H}^{*}$ by the sign of the magnetic field. In the usual bulk superconductor, the exchange of phonons leads to an effective attraction between electrons close to the Fermi surface. This interparticle potential is usually approximated by an attractive delta function with strength $g>0$; the singular nature of this potential occasionally leads to spurious divergent integrals which will be cut off at the Debye frequency. The equations of motion (4.19) have to be solved together with the self-consistency equation

$$
\Delta(\boldsymbol{r})=g F(\boldsymbol{r}, \boldsymbol{r})=(g k T / \hbar) \sum_{n} F\left(\boldsymbol{r}, \boldsymbol{r}, \omega_{n}\right)
$$

At this point, it should be noted that the all important effective attraction between electrons due to phonons may be drastically different in small particles. Low frequency phonons cannot exist in a fine particle because of its limited size; Rothwarf [188] has pointed out the possibility of enhancement of $T_{\mathrm{c}}$ in fine particles due to this low frequency cut-off. On the other hand, from a more microscopic point of view, the decrease of the number of nearest neighbour atoms on the surface causes a decrease of the force constant and therefore the average phonon frequency becomes low $[184,189]$. Of course, if the relevant mechanism for superconductivity is not only the electron-phonon interaction but if surface phonons or other surface properties are involved as well, drastic effects can be expected in small particles where the surface to bulk ratio is so favourable [179, 190-192].

In the following, we will concentrate on superconductors which are small compared with the magnetic field penetration depth and with the coherence length, but the dimensions of the systems are still large in comparison with interatomic distances. In principle, such a superconductor has the same properties as a bulk sample, as long as it is not located in a magnetic field. However, the behaviour of a small sample in a strong magnetic field has some interesting features: the gap in the one particle excitation spectrum vanishes at a certain value of the field, while the other properties of the superconducting state are retained; with increase of the field, a second order phase transition into the normal state occurs. The magnitude of this critical field depends on the dimensions of the sample and on the concentration of impurities. In our discussion, we will follow a method due to Larkin [193].

If the magnetic field is zero and the superconductor is still large compared with interatomic distances, the order parameter $\Delta$ does not depend on the size of the superconductor. This can be shown by expanding the Gor'kov relations, eqs. (4.19), in terms of the eigenfunctions of the Hamiltonian

$$
\hat{H} \psi_{\lambda}=\xi_{\lambda} \psi_{\lambda}
$$

With the expansions

$$
\begin{aligned}
& G\left(\boldsymbol{r}, \boldsymbol{r}^{\prime}\right)=\sum a_{\lambda \lambda^{\prime}} \psi_{\lambda}(\boldsymbol{r}) \psi_{\lambda^{\prime}}^{*}\left(\boldsymbol{r}^{\prime}\right) \\
& F\left(\boldsymbol{r}, \boldsymbol{r}^{\prime}\right)=\sum b_{\lambda \lambda^{\prime}} \cdot \psi_{\lambda}(\boldsymbol{r}) \psi_{\lambda^{\prime}}^{*}\left(\boldsymbol{r}^{\prime}\right)
\end{aligned}
$$


a straightforward calculation leads to

$$
\begin{aligned}
& F=\sum_{\lambda} \frac{\Delta(r)}{\hbar^{2} \omega_{n}^{2}+\zeta_{\lambda}^{2}+\Delta^{2}(r)} \psi_{\lambda}^{*}(r) \psi_{\lambda}\left(r^{\prime}\right) \\
& G=-\sum_{\lambda} \frac{i \hbar \omega_{n}+\xi_{\lambda}}{\hbar^{2} \omega_{n}^{2}+\xi_{\lambda}^{2}+\Delta^{2}(r)} \psi_{\lambda}^{*}(r) \psi_{\lambda}\left(r^{\prime}\right) .
\end{aligned}
$$

If it is now assumed that $\Delta$ is a constant, the self-consistency eq. (4.20) leads to

$$
\Delta(\boldsymbol{r})=(g k T / \hbar) \sum_{\substack{n, \lambda \\|\omega|<\omega_{\mathrm{D}}}}\left|\psi_{\lambda}(\boldsymbol{r})\right|^{2} \frac{\Delta}{\hbar^{2} \omega_{n}^{2}+\xi_{\lambda}^{2}+\Delta^{2}}
$$

If the dimensions of the system are large compared with interatomic distances so that $\Delta$ is much larger than the distance between levels, the summation in eq. (4.24) is carried out over a large number of states. The rapidly oscillating parts in $\left|\psi_{\lambda}(r)\right|^{2}$ then cancel out and this term can be replaced by the average, equal to $1 / V(V$ : volume of the particle). The assumption that $\Delta$ is not dependent on $r$ is therefore valid. The summation over $\lambda$ can be replaced by an integral over the energy $\xi$ in the usual way,

$$
\frac{1}{V} \sum_{\lambda} \cdots \simeq \rho\left(\varepsilon_{\mathrm{F}}\right) \int \mathrm{d} \xi \ldots
$$

where $\rho\left(\varepsilon_{\mathrm{F}}\right)$ is the density of states at the Fermi level. Eq. (4.24) becomes

$$
1=(g k T / \hbar) \pi \rho\left(\varepsilon_{\mathrm{F}}\right) \sum_{n}\left(\hbar^{2} \omega_{n}^{2}+\Delta^{2}\right)^{-1 / 2}
$$

which is the usual well known result for bulk superconductors [182]. Therefore one concludes that (in the absence of a magnetic field) the properties of small superconductors, which are still large on an atomic scale, are determined by the density of states at the Fermi level and by the interaction constant $g$, and are not dependent on the dimensions of the system, or the form of the boundary conditions at the surface, or the concentration of impurities.

\subsubsection{Small superconductors in a magnetic field}

4.3.2.1. Pure superconductor. If a pure small spherical superconductor with specular boundary conditions is placed in a magnetic field, the component of the angular momentum along the direction of the magnetic field is conserved. The operators $\hat{H}$ and $\hat{H}^{*}$ of eqs. (4.19) have therefore the same eigenfunctions but different eigenvalues:

$$
\hat{H} \psi_{\lambda}=\left(\xi_{\lambda}-\mu H\right) \psi_{\lambda} ; \quad \hat{H}^{*} \psi_{\lambda}=\left(\xi_{\lambda}+\mu H\right) \psi_{\lambda}
$$

Here, $\mu=\mu_{0}(e / 2 m) L$, where $\mu / \mu_{0}$ and $L$ are the components of the magnetic moment and angular momentum, and $H$ is the magnetic field. Again expanding eqs. (4.19) in terms of these eigenfunctions $\psi_{\lambda}$, one gets 


$$
F=\frac{\Delta}{\left(\hbar \omega_{n}-\mathrm{i} \mu H\right)^{2}+\xi^{2}+\Delta^{2}}, \quad G=-\frac{\mathrm{i} \hbar \omega_{n}+\xi+\mu H}{\left(\hbar \omega_{n}-\mathrm{i} \mu H\right)^{2}+\xi^{2}+\Delta^{2}}
$$

Using these solutions in the integral equation (4.20) one gets

$$
1=(g k T / \hbar) \pi \rho\left(\varepsilon_{F}\right)\left\langle\sum_{n}\left[\left(\hbar \omega_{n}-i \mu H\right)^{2}+\Delta^{2}\right]^{-1 / 2}\right\rangle
$$

Here, the brackets $\langle\ldots .$.$\rangle denote an average over all states with different values of \mu$ lying on the Fermi surface. Because of the still large dimensions of the system. on an atomic scale, one can use quasi-classical formulas for the angular momentum and the level density in the averaging process:

$$
\mu H=\mu_{0}(e / 2 m) \boldsymbol{H} \cdot(\boldsymbol{p} \times \boldsymbol{r})=\mu_{0}(e / 2 m) p_{\mathrm{F}} r H \cos \alpha .
$$

$p_{\mathrm{F}}$ is the Fermi momentum of the electron, $r$ the radial position and $\alpha=\Varangle\left(\boldsymbol{H}, \boldsymbol{p}_{\mathrm{F}}\right)$.

We will now concentrate on the case of zero temperature. Then it is necessary to replace the summation over the frequencies $\omega_{n}$ in eq. (4.29) by an integral. In order to normalize everything to the superconductor without a magnetic field, the coupling constant $g$ can be written, by using the BCS relation, as

$$
g^{-1}=\rho\left(\varepsilon_{\mathrm{F}}\right) \ln \left(2 \hbar \omega_{\mathrm{D}} / \Delta_{0}\right)
$$

where $\Delta_{0}$ is the order parameter in zero magnetic field and $\hbar \omega_{\mathrm{D}}$ the relevant phonon energy. The integral equation for the order parameter leads to an integral of the type

$$
\int_{-\infty}^{+\infty} F(\omega) \mathrm{d} \omega=\int_{-\infty}^{+\infty} \frac{\mathrm{d} \omega}{\left((\omega-\mathrm{i} \mu H)+\mathrm{i}\left(\xi^{2}+\Delta^{2}\right)^{1 / 2}\right)\left((\omega-\mathrm{i} \mu H)-\mathrm{i}\left(\xi^{2}+\Delta^{2}\right)^{1 / 2}\right)}
$$

On evaluation of this integral, two different cases have to be considered, depending on the position of the poles. If

$$
\mu_{0} \cos \alpha(e / 4 m) p_{\mathrm{F}} d H<\left(\xi^{2}+\Delta^{2}\right)^{1 / 2}
$$

or, by defining a field

$$
H_{1}=\left(4 m / \mu_{0} e\right) \Delta_{0} / p_{\mathrm{F}} d=3.26 H_{\mathrm{B}}\left(\lambda_{\mathrm{L}} / d\right)
$$

if $H \leq H_{1}$, one can displace the integration contour by $\mathrm{i} \mu H$; then the integral is no longer field dependent and one gets

$$
\Delta(H)=\Delta_{0} \quad \text { for } H \leq H_{1} .
$$

Physically, this means that the magnetic energy $\mu H$ for $H \leq H_{1}$, is not yet strong enough to break any Cooper pairs. For higher fields, i.e. if $H \geq H_{1}$, the integral equation becomes 


$$
\ln \left(\Delta / \Delta_{0}\right)=3 \int_{0}^{1}\left\{\ln \left(x u+\left\{(x u)^{2}-1\right\}^{1 / 2}\right)-\left(1-(x u)^{-2}\right)^{1 / 2}\right\} u\left(1-u^{2}\right)^{1 / 2} \mathrm{~d} u
$$

where $x=H \Delta_{0} / H_{1} \Delta$. It follows from this equation that $\Delta(H)$ goes to zero at a critical field $H_{\mathrm{c}}$ in a second order phase transition. Eq. (4.36) can be solved analytically and one gets

$$
H_{\mathrm{c}}=\frac{1}{4} \mathrm{e}^{7 / 3} H_{1}=2.57 H_{1}=8.40 H_{\mathrm{B}}\left(\lambda_{\mathrm{L}} / d\right)
$$

This critical field for a small superconducting particle, as calculated from the microscopic theory, can be compared with the results from the phenomenological approach, eqs. (4.13) and (4.18).

The excitation spectrum is given by the poles of $G$, if $\omega_{n} \rightarrow-i \omega$, leading to

$$
\varepsilon_{n}=\sqrt{\xi_{n}^{2}+\Delta^{2}}-\mu H
$$

while the density of states is given by

$$
\rho(\omega)=\sum_{n} \delta\left(\varepsilon_{n}-\hbar \omega\right)
$$

The energy gap $\hbar \omega_{\mathrm{g}}$ in the excitation spectrum is given by the state with the largest value of $\mu$ in eq. (4.38), and one gets

$$
\hbar \omega_{\mathrm{g}}=\Delta_{0}\left(1-H / H_{1}\right)
$$

Thanks to the powerful methods of Green's function techniques, it is possible to calculate explicitly all relevant physical quantities as magnetic moment, Knight shift etc. within the framework of this model of a pure superconducting particle [193].

4.3.2.2. Superconductor with impurities. If the superconducting particle contains non-magnetic impurities, it is possible to average eqs. (4.19) with respect to the random position of the impurities in exactly the same way as in the case of an infinite medium [182]. This results in a renormalization of the frequency $\omega$ and of the gap parameter $\Delta$ in terms of a relaxation time $\tau=l / v_{\mathrm{F}}$, where $l$ is the mean free path and $v_{\mathrm{F}}$ the Fermi velocity [194]. Again, this procedure is justified as long as the dimensions of the samples are large compared with interatomic distances; the corrections which arise are of the order of $\left(p_{\mathrm{F}} d / \hbar\right)$ for a small sphere of diameter $d$.

In exactly the same way as in the case of a pure superconducting particle, the critical field $H_{\mathrm{c}}$ for a second order phase transition can be found by putting $\Delta\left(H_{c}\right)=0$. In the limiting case of a very short mean free path $\left(l \ll \xi_{0}\right)$ this can be done analytically [194], and one gets at zero temperature

$$
H_{\mathrm{c}}=2.43 H_{1}\left(\xi_{0} / l\right)^{1 / 2}=7.92 H_{\mathrm{B}}\left(\lambda_{\mathrm{L}} / d\right)\left(\xi_{0} / l\right)^{1 / 2}
$$

which can be compared with the critical field of the clean case as given by eq. (4.37). The intermediate cases, $0<l<\infty$, have to be evaluated numerically [195]. Fig. 4.1 shows theoretical calculations of the critical field for a small superconducting sphere as a function of the bulk mean free path, for specular reflection of the electrons at the boundary. 


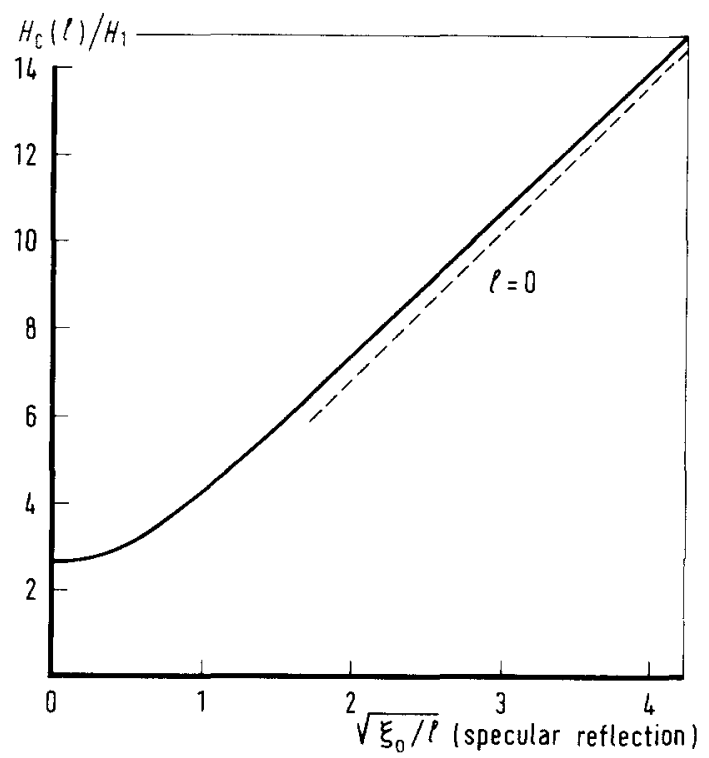

Fig. 4.1. The critical field at zero temperature, calculated for a small sphere as a function of the bulk mean free path $l$ measured relative to the coherence length $\xi_{0}$, under the assumption of specular reflection of the electrons at the boundaries. The normalization field $H_{1}$ is given by $H_{1}=\left(4 m / \mu_{0} e\right) \Delta_{0} / p_{\mathrm{F}} d=3.26 H_{\mathrm{B}}\left(\lambda_{\mathrm{L}} / d\right)$, where $H_{\mathrm{B}}$ is the bulk critical field, $\lambda_{\mathrm{L}}$ the London penetration depth, and $d$ the particle diameter. The dashed line indicates Maki's analytical solution in the limit of a small mean free path. (From Strässler and Wyder [195].)

More microscopic properties, like the density of one-particle states $\rho(\omega)$ or the electrodynamic response function $\sigma(\omega)$, can be calculated from this model with the usual formalism in a straightforward way. It has been pointed out by Maki [194] that in the limit of $l \rightarrow 0$ the theory of a superconducting particle in a magnetic field is equivalent to the celebrated theory of a superconductor with paramagnetic impurities of Abrikosov and Gor'kov [196] if $n / n_{\mathrm{c}}$ (where $n$ is the concentration of paramagnetic impurities, and $n_{\mathrm{c}}$ the critical concentration for which the metal is no longer superconducting) is replaced by $\left(H / H_{c}\right)^{2}$. The cases of finite mean free paths have to be evaluated numerically [195]. As a typical illustration, fig. 4.2 shows theoretical calculations of the density of states of a small superconducting particle in different magnetic fields for several values of the mean free path. The gap as function of the field is shown in fig. 4.3, again for different values of the mean free path as measured by the coherence length $\xi_{0}$. It is interesting to note that the field $H_{\mathrm{g}}$ at which the spectrum of excitations first becomes gapless is given by

$$
\begin{array}{ll}
H_{\mathrm{g}} / H_{\mathrm{c}}=0.954 & \text { for } l / \xi_{0} \rightarrow 0 \\
H_{\mathrm{g}} / H_{\mathrm{c}}=0.389 & \text { for } l / \xi_{0} \rightarrow \infty .
\end{array}
$$

In the latter case $H_{\mathrm{g}}$ is equal to $H_{1}$ as defined in eq. (4.34) (cf. eq. (4.37)).

Unfortunately, no real quantitative experiments on small particles exist which would allow to test in detail these theoretical predictions on the microscopic properties of small superconductors. Tunneling measurements on thin films by Millstein and Tinkham [197] seem to be in good agreement with this theory, although the theoretical results are strictly valid for small particles only. 


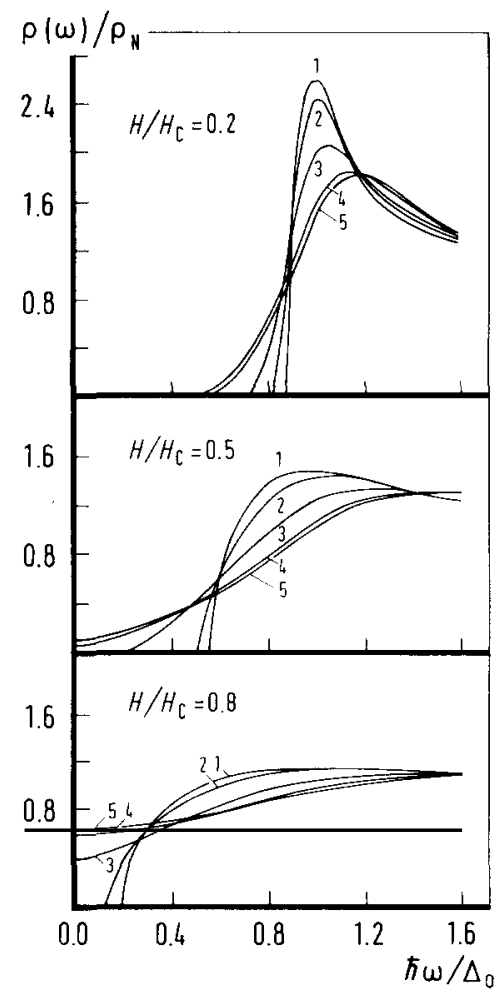

Fig. 4.2. The normalized density of states $\rho(\omega) / \rho_{\mathrm{N}}$ as a function of energy $\hbar \omega$ for a superconducting small sphere in a magnetic field $H$, calculated for different mean free paths at zero temperature. $\rho_{\mathrm{N}}$ is the density of states in the normal phase, $H_{\mathrm{c}}$ is the critical field of the small particle, and $\Delta_{0}$ is the bulk BCS order parameter at $H=0$ and $T=0 ; l$ is measured relative to the coherence length $\xi_{0}(1: l=0 ; 2$ : $\left.l=(\pi / 10) \xi_{0} ; 3: l=\pi \xi_{0} ; 4: l=(10 \pi) \xi_{0} ; 5: l=\infty\right)$. (From Strässler and Wyder [195].)

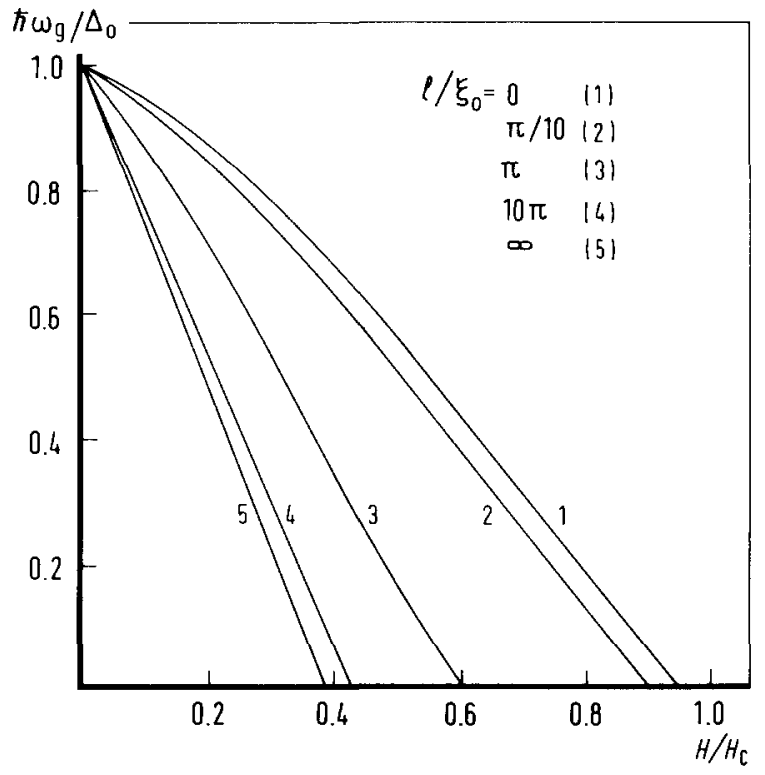

Fig. 4.3. The normalized energy gap $\hbar \omega_{\mathrm{g}} / \Delta_{0}$ of a small superconducting particle in a magnetic field $H$, calculated for different values of the mean free path at zero temperature. $H_{c}$ is the critical field of the small particle and $\Delta_{0}$ is the bulk BCS order parameter at $H=0$ and $T=0 ; l$ is measured relative to the coherence length $\xi_{0}$. (From Strässler and Wyder [195].)

4.3.2.3. Trajectory method. In the calculation of the critical field $H_{\mathrm{c}}$ by expanding eqs. (4.19) in eigenfunctions of the Hamiltonian $\hat{H}$, it was implicitly assumed that the electrons are specularly reflected at the boundaries of the particle. De Gennes and Tinkham [198] have discovered a method which allows to calculate critical fields for the more realistic cases where the electrons are scattered diffusely at the boundaries.

This method starts from the Gor'kov relations, eqs. (4.19) and (4.20), as well. It is assumed that the order parameter $\Delta$ is spatially uniform, and that there is a second order phase transition at $H_{c}$ $\left(\Delta\left(H_{c}\right) \rightarrow 0\right)$. The linearized Gor'kov relation, valid for $\Delta \rightarrow 0$, is given by

$$
\Delta^{*}(r)=(g k T / \hbar) \sum_{n} \int \mathrm{d} \boldsymbol{r}^{\prime} G\left(\boldsymbol{r}, \boldsymbol{r}^{\prime}, \omega_{n}\right) \Delta^{*}\left(\boldsymbol{r}^{\prime}\right) G\left(\boldsymbol{r}, \boldsymbol{r}^{\prime},-\omega_{n}\right)
$$

where $G\left(r, r^{\prime}, \omega\right)$ is the Green's function of the normal material. The kernel of this integral equation can be expressed in terms of one-electron correlation functions in the normal state [199]. Explicitly one 
gets

$$
G\left(r, r^{\prime}, \omega\right) G\left(r, r^{\prime},-\omega\right)=2 \pi \rho\left(\varepsilon_{\mathrm{F}}\right) V \int_{0}^{\infty} \mathrm{d} t \mathrm{e}^{-2|\omega| t} f\left(r, r^{\prime}, t\right)
$$

where $V$ is the sample volume and

$$
f\left(\boldsymbol{r}_{1}, \boldsymbol{r}_{2}, t\right)=\left\langle\exp \left(\frac{2 \pi \mathrm{i}}{\Phi_{0}} \int_{\boldsymbol{r}_{1}}^{\boldsymbol{r}_{2}} \boldsymbol{A} \cdot \mathrm{d} \boldsymbol{l} \delta\left(\boldsymbol{r}(0)-\boldsymbol{r}_{1}\right) \delta\left(\boldsymbol{r}(t)-\boldsymbol{r}_{2}\right)\right)\right\rangle
$$

where $\Phi_{0}=h / 2 e$ is the flux quantum. The integral in this equation,

$$
\phi(t)=2 \pi \int_{r_{1}}^{r_{2}} \frac{\boldsymbol{A} \cdot \mathrm{d} l}{\Phi_{0}}
$$

is taken along the classical trajectory linking the points $\boldsymbol{r}_{1}$ and $\boldsymbol{r}_{2}$ in a time interval $t$. Eq. (4.45) is valid only if the magnetic field is so weak that the electron trajectory can be considered as a straight line, i.e. the curvature radius must be large compared with the coherence length $\xi_{0}$. In a gauge where $\Delta$ is real and constant, one gets for the integral equation determining $H_{\mathrm{c}}(T)$

$$
1=2 \pi k T \rho\left(\varepsilon_{\mathrm{F}}\right) g \sum_{\omega} \int_{0}^{\infty} \mathrm{d} t \mathrm{e}^{-2|\omega| t}\left\langle\mathrm{e}^{\mathrm{i} \phi(t)}\right\rangle
$$

The average $\langle\ldots\rangle$ is taken over all initial positions and all initial orientations of the velocity for one electron in the sample. The problem is now reduced to the study of all classical one-electron trajectories in the normal state.

As discussed in detail by de Gennes and Tinkham [198], two types of magnetic behaviour have to be distinguished:

$$
\begin{array}{ll}
\text { case I: } & \lim _{t \rightarrow \infty}\left\langle\mathrm{e}^{\mathrm{i} \phi(t)}\right\rangle=\eta \neq 0 \\
\text { case II: } & \lim _{t \rightarrow \infty}\left\langle\mathrm{e}^{\mathrm{i} \phi(t)}\right\rangle=\exp \left(-t / \tau_{K}\right) .
\end{array}
$$

Here, $\eta$ and $\tau_{K}$ are functions of the applied field $H$, and the subscript $K$ makes reference to the time reversal operator $K$ (cf. section 3.3.2).

In case I, eq. (4.47) takes the usual BCS form [181] and one gets

$$
k T_{\mathrm{c}}(H)=1.14 \hbar \omega_{\mathrm{D}} \exp \left\{-1 / \rho\left(\varepsilon_{\mathrm{F}}\right) g \eta(H)\right\}
$$

Therefore, case I leads to a conventional superconductor, except for the fact that the coupling constant 
$\rho\left(\varepsilon_{\mathrm{F}}\right) g \eta(H)$ is a decreasing function of the applied field $H$; the transition temperature $T_{\mathrm{c}}$ remains finite at all fields but becomes exponentially small for small $\eta(H)$. A similar result has been found by Nambu and Tuan $[200,201]$ using more conventional techniques.

If the exponential decay law of case II is already obeyed at the times $t$ of interest, one gets

$$
1=2 \pi k T \rho\left(\varepsilon_{\mathrm{F}}\right) g \sum_{\omega} \frac{1}{2|\omega|+1 / \tau_{K}}
$$

and this leads to

$$
\ln \left(\frac{T_{\mathrm{c} 0}}{T_{\mathrm{c}}(H)}\right)=\Psi\left(\frac{1}{2}\right)-\Psi\left(\frac{1}{2}+\frac{\hbar}{4 \pi k T_{\mathrm{c}} \tau_{\mathrm{K}}}\right)
$$

$T_{\mathrm{c} 0}$ is the transition temperature without a field, $T_{\mathrm{c}}(H)$ the transition temperature with the field, and $\Psi$ is the di-gammafunction defined by $\Psi(z)=\Gamma^{\prime}(z) / \Gamma(z) . T_{\mathrm{c}}\left(\tau_{K}\right)$ decreases with decreasing $\tau_{K}$ and vanishes for

$$
1 / \tau_{K}=1.76 k T_{\mathrm{co}} / \hbar
$$

Eq. (4.51) is identical to the result for the critical temperature of a superconductor with paramagnetic impurities [196].

A simple example of case II is a small particle of dimension $d$ much larger than the mean free path $l$. Note that in this case the condition that $|\Delta|$ is constant, imposes an upper bound on $d$; for larger values of $d$ the order parameter is not spatially uniform any more, and superconductivity nucleates at a field $H_{\mathrm{c} 2}$ where the nuclei have a size of the order of $\left[\xi_{0} l\left(T_{\mathrm{c} 0} /\left(T_{\mathrm{c} 0}-T\right)\right)\right]^{1 / 2}$. Therefore, we must have

$$
l<d<\left(\xi_{0} l \frac{T_{\mathrm{c} 0}}{T_{\mathrm{c} 0}-T}\right)^{1 / 2}
$$

allowing only a rather narrow range of $d$. From the point of view of the trajectories, the electron will have explored all regions of the sample at a time $t$. The phase $\phi(t)$ is therefore the sum of many uncorrelated increments and one has

$$
\left\langle\mathrm{e}^{\mathrm{i} \phi(t)}\right\rangle=\mathrm{e}^{-\left\langle\phi^{2}(t)\right\rangle / 2}=\mathrm{e}^{-t / \tau K} \quad\left(\text { for } t \geq \tau=l / v_{\mathrm{F}}\right) .
$$

Carrying out the detailed calculations, one gets

$$
\frac{1}{\tau_{K}}=\frac{1}{3} \tau\left(\frac{2 e v_{\mathbf{F}}}{h}\right)^{2}\left\langle\boldsymbol{A}^{2}(r)\right\rangle
$$

Here, $\tau$ is a transport mean lifetime, and the average $\langle\ldots\rangle$ is over the volume of the sample. As usual in this sort of problems, the choice of the gauge in eq. (4.55) is imposed by the condition of $\Delta=$ constant. By inspection one finds for a spherical grain of diameter $d$ in a uniform field

$$
\boldsymbol{A}=\frac{1}{2} \boldsymbol{r} \times \boldsymbol{B}
$$


and a value of

$$
\left\langle A^{2}\right\rangle=B^{2} d^{2} / 40
$$

A nearly equivalent result has been obtained by Maki [194] through an approximate direct solution of the Gor'kov relations, eqs. (4.19) and (4.20).

For a small particle with few imperfections $(l \gg d)$, it is important to define how the electrons are scattered at the boundary. If this scattering is specular, one finds the situation of case I; this case is more transparently treated using Larkin's method as discussed in section 4.3.2.1. If the scattering of the electrons on the surface is diffuse, there is again a non-zero random increment in phase between successive collisions on the surface and the behaviour corresponds to case II. The corresponding $\tau_{K}$ is now given by [198]

$$
\frac{1}{\tau_{K}}=\frac{\pi^{2}}{180}\left(\frac{B}{\Phi_{0}}\right)^{2} v_{\mathrm{F}} d^{3}
$$

This leads to a critical field at $T=0$ of

$$
H_{\mathrm{c}}(0)=13.7 H_{\mathrm{B}}\left(\lambda_{\mathrm{L}} / d\right)\left(\xi_{0} / d\right)^{1 / 2}
$$

Comparing eq. (4.59) with eq. (4.41), valid for a finite mean free path $l$, we see that the two results agree if we define an "effective" mean free path $l_{0}=0.34 d$ due to the diffuse boundary reflection. The diffuse boundary condition acts like an additional scattering mechanism, as is well known from size effect studies. Therefore, the theoretical results for critical fields for different mean free paths (calculated for specular reflection of the electrons at the boundaries) can be used for the case of diffuse scattering as well, if the mean free path is properly adjusted.

4.3.2.4. Experimental investigations. Unfortunately, not very many quantitative experimental investigations of the theoretical relations discussed in section 4.3 .2 do exist. Up to rather recently, the only detailed experimental studies dealt with the magnetic properties of superconducting mercury colloids and were done by Shoenberg and collaborators [202,203]. Due to the lack of appropriate theories, these results were interpreted in a more qualitative way, using the sort of London theory as sketched in section 4.2, combined with a Gorter-Casimir two fluid model. The advances of the more microscopic theory have triggered new experimental investigations of the magnetization of small superconducting particles [204,205]. Fig. 4.4 shows the magnetic moment observed by Morozov and coworkers [204] on a collection of small tin particles at temperatures between $3.57 \mathrm{~K}$ and $3.09 \mathrm{~K}$. The full curves represent the theoretical prediction, based on Ovchinnikov's [206] straightforward extension of Larkin's model as discussed in section 4.3.2.1. In the theoretical curves, the measured particle size distributions have properly been taken into account.

Probably the most interesting experimental investigations on the critical fields of small superconductors have been carried out by Zeller and Giaever [207,208]. They prepared tunnel junctions which contain small Sn particles embedded in the oxide barrier, and by measuring the tunneling characteristic of these junctions they were able to measure the critical fields of the small Sn particles. For particles with a diameter $d>20 \mathrm{~nm}$, their experimental results are in good agreement with eq. (4.41) or (4.59), with proper definition of the mean free path. However, for particles with $d<20 \mathrm{~nm}$, the critical field 


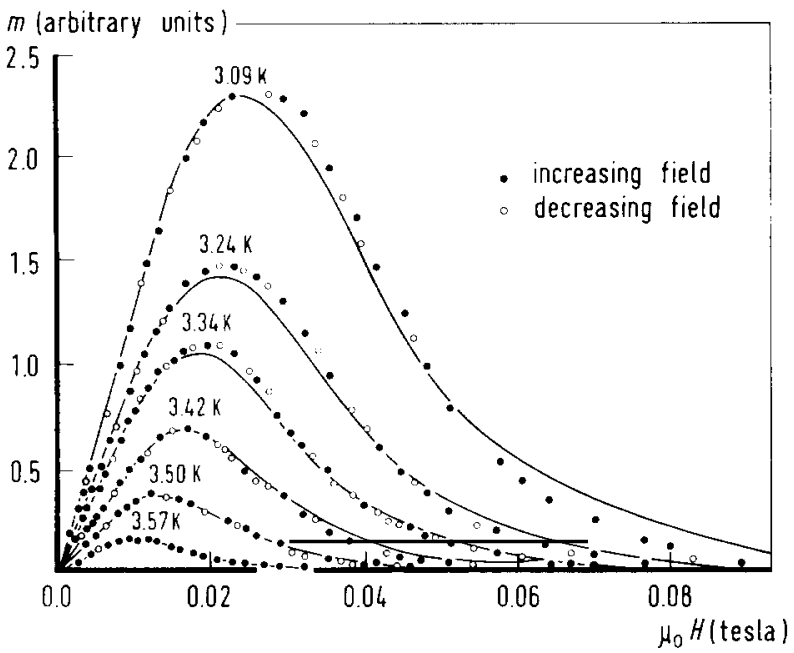

Fig. 4.4. Magnetic moment $m$ of a collection of isolated tin particles with a mean diameter of $d=130 \mathrm{~nm}$ as a function of the magnetic field. The full curves represent the result of theoretical calculations, based on an extension of Larkin's microscopic model, and taking into account the measured particle size distribution. (From Morozov, Naumenko and Petinov [204].)

goes up much faster than the theory predicts (see fig. 4.5). No indication of the Clogston paramagnetic limit, as discussed in section 4.2 , was found, as the small particles were still superconducting in fields of $10 \mathrm{~T}$ at $4.2 \mathrm{~K}$, while the Clogston formula would give for $\mathrm{Sn}$ a maximum critical field of $\mu_{0} H_{\mathrm{c}}^{\max }(0)=$ $6.8 \mathrm{~T}$. It is possible that the paramagnetic limit is much higher than predicted by the simple BCS theory due to the non-vanishing Knight shift, as indicated by Wright's [209] measurements. However, it should be emphasized that this drastic increase of the critical field with decreasing particle size is unexplained

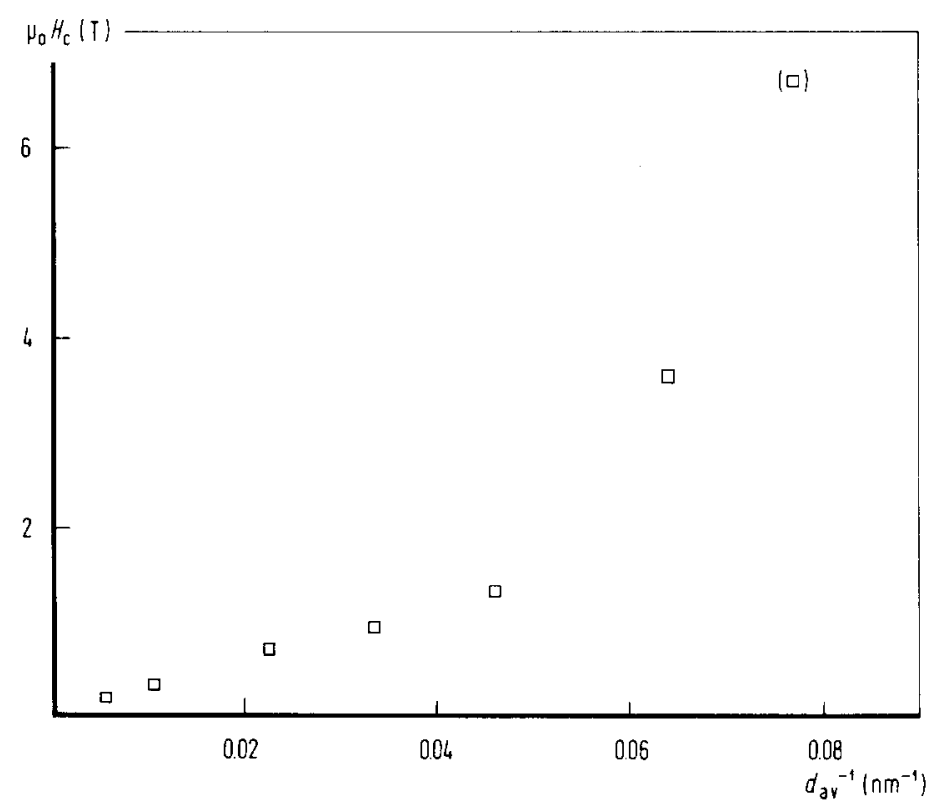

Fig. 4.5. Critical field $H_{\mathrm{c}}$ as a function of the inverse average particle diameter $\boldsymbol{d}_{\mathrm{av}}^{-1}$ for small superconducting tin particles, embedded in the oxide layer of a tunnel junction, as measured by tunneling. The field was applied parallel to the plane of the tunnel junction. The symbol between parentheses corresponds to a conservative estimate. (From Zeller and Giaever [207].) 
as yet; the effect is probably not related to QSE, because these effects (as seen in gap measurements with tunneling $[207,208]$ ) only start showing up for much smaller particles, when $d \leq 10 \mathrm{~nm}$ (see section 4.3.3).

\subsubsection{Quantum size effects in small superconducting particles}

If the superconducting particles are getting so small that more microscopic distances come into play, it is to be expected that the basic principles for superconductivity itself are influenced by size effects, and the question arises if there exists a lower limit in size for particles still to be superconducting. For such very small particles, not only the electronic properties will be influenced, but the phonon spectrum (considered to be responsible for superconductivity) will certainly be changed as well [210,211]; in addition, because of the favourable surface to volume ratio in small particles, also possible electronelectron interaction via surface phonons or other surface effects should be considered [179, 212]. Here, we will concentrate on the influence of the particle size on the electron system only.

It has been conjectured by Anderson [213] some time ago, that the usual Cooper instability will not exist any more and therefore superconductivity should disappear if the small superconducting particles are in the quantum size effect regime with the energy difference between two discrete one electron states comparable to the energy gap of the superconducting state (Anderson criterion). This means that small superconductors with fewer than about $10^{4}$ to $10^{5}$ electrons should be affected by this effect.

This question is related to theoretical studies by Blatt and Thompson [214,215] on thin superconducting films. These authors used electronic wave functions which are spatially quantized, perpendicular to the plane of the film, to perform the BCS pairing. Due to this size quantization in one direction one gets sharp and pronounced "shape resonances" in the order parameter $\Delta$ of the superconductor as a function of the width of the slab. This quantum size effect has been observed with tunneling experiments on tin by Komnik, Bukhshtab and Man'kovskii [216]. The relevant situation in small particles has been studied by Shapoval [217], and in greater detail by Parmenter [218].

As usual, one has to start from the Gor'kov relations, eqs. (4.19) and (4.20). The transition temperature can be found from the self-consistency equation

$$
\Delta(r)=(g k T / \hbar) \sum_{\omega} F(r, r, \omega)
$$

where the bulk cut-off frequency $\omega \simeq \omega_{\mathrm{D}}\left(\omega_{\mathrm{D}}\right.$ : Debye frequency) will be used. Again using Larkin's [193] method of expanding the Gor'kov equations in terms of the one-electron normal state eigenfunctions $\psi_{\lambda}$ of the Hamiltonian, eq. (4.24) can be used to study the influence of QSE on superconductivity:

$$
\Delta(\boldsymbol{r})=(g k T / \hbar) \sum_{\substack{n, \lambda \\|\omega|<\omega_{\mathrm{D}}}}\left|\psi_{\lambda}(\boldsymbol{r})\right|^{2} \frac{\Delta}{\hbar^{2} \omega_{n}^{2}+\frac{\zeta^{2}}{\zeta_{\lambda}}+\Delta^{2}} .
$$

Substituting a constant value of the order parameter on the right hand side, one gets

$$
\Delta(\boldsymbol{r})=g \rho\left(\varepsilon_{\mathrm{F}}\right) \ln \left(\frac{2 \hbar \omega_{\mathrm{D}}}{\pi k T_{\mathrm{c}}} \mathrm{e}^{\gamma}\right) \Delta\left\langle\left|\psi_{\lambda}(\boldsymbol{r})\right|^{2}\right\rangle
$$

where $\mathrm{e}^{\gamma} \simeq 1.781$. The square of the wavefunction $\left|\psi_{\lambda}(r)\right|^{2}$ is averaged over all states near the Fermi 
surface; when boundary effects are neglected, this average is equal to $1 / V$, as discussed in section 4.3.1.

If boundary effects are taken into account, $\left|\psi_{\lambda}(r)\right|^{2}$ has to decrease near the boundary of the particle over a distance of the order of the de Broglie wavelength of the conduction electrons $\left(\simeq \hbar / p_{\mathrm{F}}\right)$ and it vanishes at the surface of the sample. As the integral of $\left|\psi_{\lambda}(r)\right|^{2}$ over the volume of the particle remains the same, the mean square inside the sample is increased to

$$
\left\langle\left|\psi_{\lambda}(r)\right|^{2}\right\rangle=\left(V-\alpha \hbar S / p_{\mathrm{F}}\right)^{-1}
$$

where $V$ and $S$ are the volume and the surface of the sample and $\alpha$ a numerical constant of order unity. Shapoval [217] argues that, in addition, the Fermi level $\varepsilon_{\mathrm{F}}=p_{\mathrm{F}}^{2} / 2 m$ is shifted due to the presence of the boundary conditions; the effective volume, i.e. the region accessible to the electrons is now, in accordance with eq. (4.63), smaller by an amount $\alpha \hbar S / p$. This leads to a shift of the Fermi momentum $p_{\mathrm{F}}$ given by

$$
\int_{0}^{p_{F}}\left(1-\frac{\alpha \hbar S}{p V}\right) p^{2} \mathrm{~d} p=\int_{0}^{p_{0}} p^{2} \mathrm{~d} p
$$

where $p_{0}$ is the Fermi momentum in the bulk material. Therefore

$$
\rho=\rho_{0}\left(1-\alpha \hbar S / 2 p_{0} V\right)
$$

where $\rho_{0}$ is the density of states for a sample of infinite size. If both these effects are taken into account, an effective density of states can be introduced by

$$
\rho_{\text {eff }}=\rho\left\langle\left|\psi_{\lambda}\right|^{2}\right\rangle V=\rho_{0}\left(1+\alpha \hbar S / 2 p_{0} V\right)
$$

which can be interpreted as a density of states near the Fermi surface divided by an effective volume. The final equation for the transition temperature $T_{\mathrm{cp}}$ for the small particle can now be written in the BCS form as

$$
1=g \rho_{\mathrm{eff}} \ln \left(\frac{2 \hbar \omega_{\mathrm{D}}}{\pi k T_{\mathrm{cp}}} \mathrm{e}^{\gamma}\right)
$$

and from this one gets the increase $\Delta T$ of the transition temperature compared with the transition temperature $T_{\mathrm{c}}$ of the bulk material as

$$
\frac{\Delta T}{T_{\mathrm{c}}}=\frac{\alpha \hbar S}{2 p_{0} V} \ln \left(\frac{2 \hbar \omega_{\mathrm{D}}}{\pi k T_{\mathrm{c}}} \mathrm{e}^{\gamma}\right)
$$

For small particles of ordinary metals with dimensions of the order of $10 \mathrm{~nm}$, this formula predicts an increase of 10 to $15 \%$ in the critical temperature.

Using the same sort of ideas, Parmenter [218] has calculated, in addition to the transition temperature, the energy gap of a small particle of a superconductor in the QSE regime. A small superconducting cube with cube edge $L$ is studied within the framework of the usual BCS theory, and 
now instead of the continuous one-electron energy spectrum, the discrete QSE-spectrum is used. Sums of the form $\Sigma_{k} \psi(k)$ can be rewritten as

$$
\sum_{k} \psi(k)=\left(\frac{L}{2 \pi}\right)^{3} \sum_{\nu} \int_{\infty} \psi(k) \exp (-\mathrm{i}(\boldsymbol{\nu} \cdot \boldsymbol{k}) L) \mathrm{d}^{3} k
$$

where

$$
\nu=e_{x} \nu_{x}+e_{y} \nu_{y}+e_{z} \nu_{z}
$$

$\boldsymbol{e}_{\alpha}$ being the unit vectors along the coordinate axes, and $\nu_{\alpha}$ being any integer, positive, negative or zero. Eq. (4.69) can be evaluated by an application of the Poisson sum formula. For a bulk superconductor, all the terms in the summation over $\nu$ are negligible, except for $\nu=0$. As the particle size is reduced, the terms in eq. (4.69) with finite $\nu$ represent the correction to the BCS theory due to the QSE. The geometry of a cube considered here leads to the well known artificial degeneracy, all terms with $\nu \neq 0$ will have sinusoidal phases that change rapidly as a function of $L p_{F} / \hbar$. For values of $L$ in the order of tens of nanometers, it seems reasonable to assume that this rapid variation with change in size is an artefact of the highly symmetrical geometry of the cube, and that therefore they can be averaged with respect to this rapid change of phase in the various sums over $k$, as they appear in the usual BCS expression. The rest of the calculations is straightforward and follows closely the conventional BCS method. According to Parmenter [218], the QSE leads to corrections to the BCS results in a sense that formally the weak coupling limit goes over into a strong coupling limit. Following these calculations, it is possible to introduce a characteristic length $d_{\mathrm{A}}$ [218], defined by

$$
d_{\mathrm{A}}=\left(\hbar^{2} \xi_{0} / p_{\mathrm{F}}^{2}\right)^{1 / 3}
$$

where $\xi_{0}$ is the Pippard coherence length at $T=0$ and $\hbar / p_{\mathrm{F}}$ the de Broglie wavelength of the electrons at the Fermi surface. For dimensions $d \leq d_{\mathrm{A}}$, QSE should come into play. Essentially, $d_{\mathrm{A}}$ is again the dimension of a small particle where the Anderson relation is fulfilled: The order parameter of the superconductor is equal to the QSE energy level splitting, $\Delta=\delta\left(d_{\mathrm{A}}\right)$. This type of QSE in the properties of small superconductors can therefore be expected if the diameter $d$ of the small particle is comparable to $d_{\mathrm{A}}$ :

$$
d \simeq d_{\mathrm{A}}
$$

As an example, one gets $d_{\mathrm{A}} \simeq 6 \mathrm{~nm}$ for aluminium. It seems not unlikely that observations of $T_{\mathrm{c}}$-enhancement in granular films [190-192,219-223] can be explained on the basis of this sort of theories, although real quantitative comparisons between theory and experiment are missing, and changes in the phonon spectrum (such as surface phonons, changes of the phonon spectrum due to the size effect, etc.) should be considered as well.

It should be noted that, in the usual BCS-form of the free energy, many-electron wave functions appear which are not eigenfunctions of the total electron number. This might conceivably lead to problems in very small systems that contain few conduction electrons, and where only a few oneelectron energy levels are present in the energy range where the BCS-pairing occurs [224]. 
Markowitz [225] has studied theoretically superconductivity in small particles in relation with charge fluctuations. This approach is related to Kubo's consideration about charge neutrality in normal-state QSE systems (see section 3.2.1). To have maximum correlation energy in a superconductor, the phase of the superconductor should be a constant. However, due to the uncertainty relation, this constant phase produces infinitely large fluctuations in the relative number of electrons; on the other hand, for electrostatic reasons, a uniform charge density distribution would be energetically most favourable. Since the superconductor cannot have both maximum correlation energy and minimum Coulomb repulsion energy, the two have to be optimized. For small isolated particles, this Coulomb term becomes important; it was calculated numerically by Markowitz that one should get occupied singlet states in the ground state of a small superconducting sphere with a diameter of about $100 \mathrm{~nm}$, and superconductivity should be destroyed when the particles become smaller than $20 \mathrm{~nm}$, because of this Coulomb repulsion term. Obviously, this type of theory can only be applied to systems of small particles where the small particles are highly isolated from each other so that charge exchange is prohibited.

From an experimental point of view, the situation around the existence of QSE in the properties of small superconducting particles is not very transparent. There seems to be a general agreement that ultimately some size effect will quench superconductivity; it is hard to imagine that a bead consisting of ten atoms should still be a superconductor, simply because there are not enough electrons left. There can be no doubt that in this size region the usual bulk theory of superconductivity will lose its meaning; the question of a lower size limit for superconductivity depends then crucially on the definition of superconductivity itself. In addition, it is obvious from thermodynamics that the transition from the normal to the superconducting state is washed out if the condensation energy becomes comparable to the thermal energy $k T[207,208]$, i.e. if

$$
\frac{1}{2} \mu_{0} H_{\mathrm{c}}^{2} V \lesssim k T_{\mathrm{c}}
$$

where $H_{\mathrm{c}}$ is the bulk critical field and $k T_{\mathrm{c}}$ is the condensation energy of a single electron. Obviously, due to the intimate relationship of the order parameter $\Delta$, density of states $\rho$, critical temperature $T_{c}$, critical field $H_{c}$, volume of the sample $V=\frac{1}{6} \pi d^{3}$, and QSE parameter $\delta$, the criterion eq. (4.73) is again, as in eq. (4.72), equivalent to stating that superconductivity should disappear if the energy gap is equal to the QSE energy splitting (Anderson criterion [213]):

$$
\Delta \leq \delta
$$

This means that at low temperatures the thermal fluctuations have to be either small compared with the condensation energy for large particles, or with the QSE electron level spacing for small particles. Therefore, at low temperatures, the particle should be either superconducting, or in the QSE regime.

The classical experiments on small superconducting particles have been carried out quite some time ago by Shoenberg [202] on the magnetization and by Reif [226] on the Knight shift in superconductors. Although in both studies the particle size was in a regime, where Markowitz's electrostatic effects should be important, no such effects have been detected. Apart from magnetic field effects, all of these results can be discussed mainly in the framework of the usual theory of the bulk superconductor. Despite quite some intensive and careful work (mainly on $T_{\mathrm{c}}, H_{\mathrm{c}}$, and NMR in small particles $[116-120,125,126,128,184])$, no coherent picture of the experimental situation seems to emerge. The most beautiful and interesting investigations into the microscopic properties of small superconducting particles have been carried out by Zeller and Giaever [207,208]. They have studied small metallic 
particles of $\mathrm{Sn}$ in the size range of $5 \mathrm{~nm} \leq d \leq 200 \mathrm{~nm}$. The experimental arrangement was such that the Sn particles were evaporated onto the insulating oxide of a tunnel junction; the Sn agglomerated into small particles, and by carefully controlling the oxidation process, the space between the particles was filled with a rather thick oxide which embedded the particles inside the tunnel junction. Contact with the particles was only made by electron tunneling. Most of the electrons flowing across the junction tunneled into and out of one of the embedded particles. As the transmission coefficient was of the order of $10^{-10}$, the particles can be considered as being insulated from the film electrodes and from each other. This ingenious technique does not only allow to check if a particle is superconducting or not (i.e. to measure the critical fields of small particles, see section 4.3.2.4), it is also possible to measure directly the energy gap of the superconductor with tunneling (fig. 4.6). For Sn, the Anderson criterion of eq. (4.74) leads to a critical diameter $d_{\mathrm{A}} \simeq 5 \mathrm{~nm}$, while the charge fluctuations of Markowitz should influence the superconductive behaviour for $d \leq 100 \mathrm{~nm}$ and should destroy superconductivity for $d \simeq 20 \mathrm{~nm}$ (Markowitz's calculations apply for completely free particles and should be modified for this case, since the Coulomb interaction is screened by the oxide in which the particles are embedded). Zeller and Giaever have found that the energy gap in small Sn particles is only slightly increased from the bulk value, and almost size independent down to a particle diameter of $d \simeq 10 \mathrm{~nm}$. Particles with a diameter between 6 and $10 \mathrm{~nm}$ still show a gap, but the gap is smeared out and not much information could be extracted from the measurements. Fig. 4.7 shows the measured size dependence of the energy gap compared with Parmenter's calculation based on the QSE; it is obvious that the Parmenter mechanism can be ruled out as the dominant mechanism for particles with $d>16 \mathrm{~nm}$. The experiments

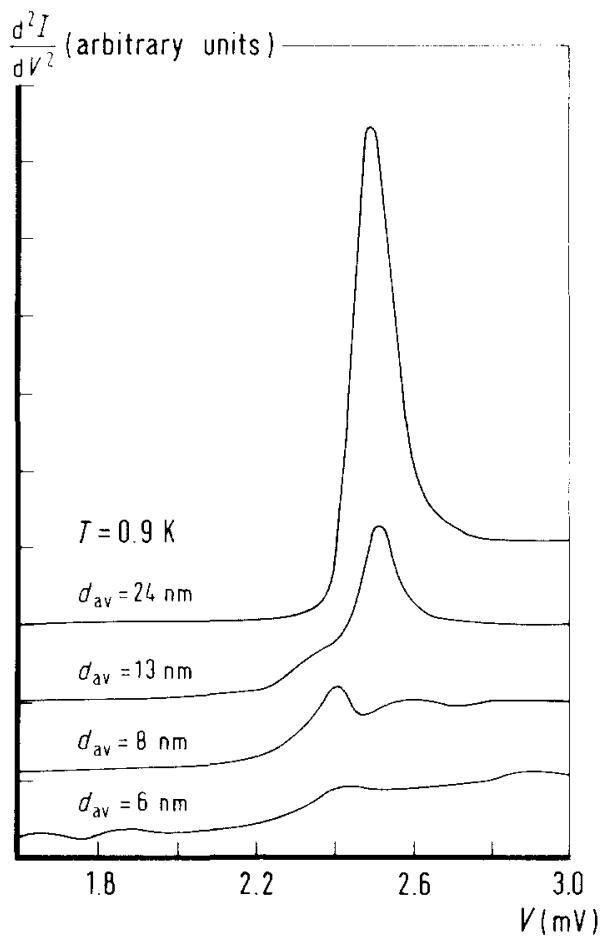

Fig. 4.6. Size dependence of the energy gap $\Delta$ in small superconducting particles. The figure shows the second derivative $\mathrm{d}^{2} I / \mathrm{d} V^{2}$ of the tunneling characteristic of tin film junctions, with tin particles of an average diameter $d_{\mathrm{av}}$ embedded in the oxide layer. For the different curves, the zero of the vertical scale has been offset in steps of one unit. Junctions with particles with a diameter $d_{\mathrm{av}}<6 \mathrm{~nm}$ did not show any structure which could be attributed unambiguously to an energy gap. (From Zeller and Giaever [208].) 


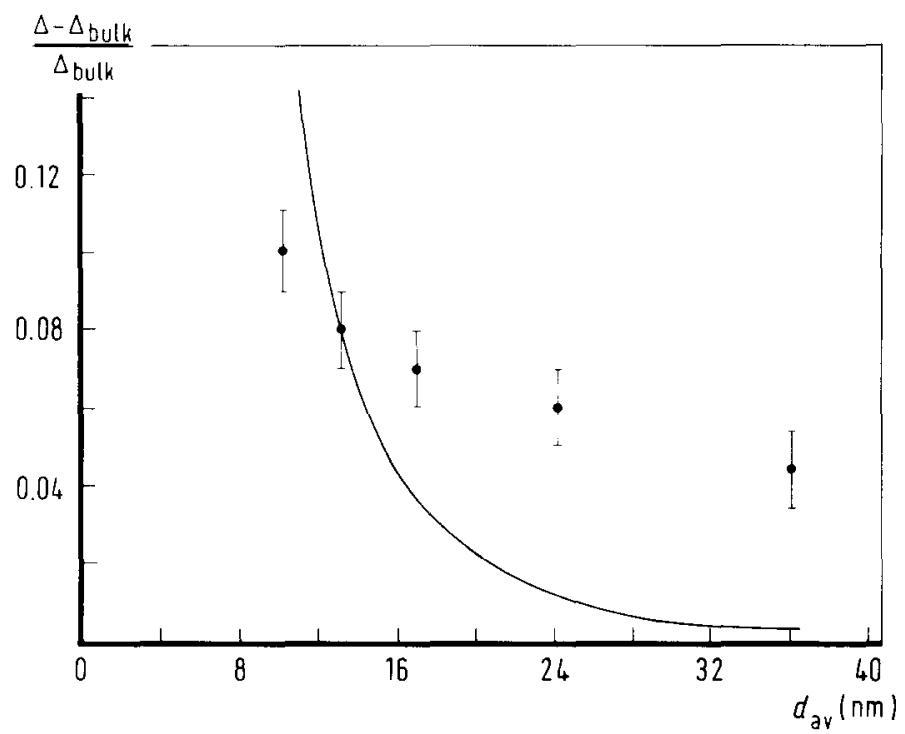

Fig. 4.7. Zeller and Giaever's measurements of the energy gap $\Delta$ of small superconducting particles as a function of the average diameter $d_{\mathrm{av}}$ of the particles. The measurements were done by tunneling on tin film junctions, with the tin particles embedded in the oxide layer. The solid line is calculated from Parmenter's theory based on QSE; in these calculations, the dimension, for which the spacing $\delta$ between two QSE energy levels becomes equal to the bulk energy gap $\Delta_{\text {bulk }}$, is assumed to be $d=5 \mathrm{~nm}$. (From Zeller and Giaever [208].)

by Zeller and Giaever show that superconductivity persists in particles down to the size given by the Anderson criterion $d_{\mathrm{A}}$; a lower size limit for superconductivity could not yet be determined. In the tunneling characteristic of particles with $d<6 \mathrm{~nm}$, no structure which could be attributed to an energy gap was observed. This does not necessarily mean that such particles are not superconducting, it can be explained by a smearing out due to a strong size dependence of the energy gap. The Markowitz charge fluctuation mechanism can be ruled out (which is probably not too surprising since the Coulomb interaction is partially screened by the matrix in which the particles are embedded), and the Parmenter QSE seems not to explain the results in quantitatively satisfactory way. However, there is no doubt that something very interesting happens to the superconductivity of particles with a size $d \leq d_{\mathrm{A}}$; the properties related to superconductivity start changing drastically as can be seen from the increasing energy gap and the unexpected high rise of the critical magnetic field with decreasing particle size (see section 4.3.2.4).

\subsection{Fluctuations in small superconducting particles}

As the characteristic correlation length given by eq. (4.2) or eq. (4.3) is rather long, the thermodynamic fluctuations in superconductors are generally quite small; in most cases, the transition temperature $T_{c}$ can be considered to mark a sharp dividing point between "normal" and "superconducting" behaviour. However, in small particles the smallness of the dimensions of the particles will drastically reduce the coherence length $\xi_{\Delta}$, and fluctuations should be visible very clearly. An excellent review on the whole field of fluctuations in superconductors has been published by Skocpol and Tinkham [227]. Therefore, here we will limit our discussion to the essential points only, relevant to small particles.

As the modern theory of superconductivity is a mean field theory, the basic problems on fluctuations 
can most clearly be seen in the well known textbook solutions of the Ising model [228, 229]. As the final results are independent of the specific structure of the Ising model, they can be used for almost all second order phase transitions, including superconductivity. We will follow closely Strässler's masterly tutorial [230] on these problems.

The Ising model essentially describes a very anisotropic ferromagnet in which only the $z$-components of the spins are coupled. Let the spins at the lattice point $r$ be denoted by $\sigma_{r}$; each $\sigma_{r}$ can take the values +1 or -1 . Then in the usual dimensionless units, the Hamiltonian is given by

$$
\hat{H}=-\sum_{r} B(r) \sigma_{r}-\frac{1}{2} \sum_{r, r^{\prime}} J_{r-r^{\prime}} \sigma_{r} \sigma_{r^{\prime}}
$$

where the first term represents an interaction with an external magnetic field $B(r)$ which is allowed to vary with $r$ for mathematical reasons. The second term represents an attractive interaction between spins, and contributes an energy $-J_{\boldsymbol{r}-\boldsymbol{r}}$ if the spins point in the same direction or $+J_{\boldsymbol{r}-\boldsymbol{r}}$ if they point in opposite directions. In the mean field approximation the problem is reduced to the behaviour of one single spin in the averaged effective field:

$$
B_{\mathrm{eff}}=B+\langle\sigma\rangle \sum_{\rho} J_{\boldsymbol{\rho}}
$$

where $\langle\ldots\rangle$ denotes the thermodynamical average. The magnetization of a spin in an effective field is then given by

$$
\langle\sigma\rangle=\tanh \left(B_{\mathrm{eff}} / T\right)
$$

which solves the problem within the mean field approximation. In order to study the influence of fluctuations on different physical quantities, one usually introduces the "random phase approximation" as a trivial generalization of the mean field approximation by allowing spatial variation of the effective field:

$$
B_{\text {eff }}(\boldsymbol{r})=B(\boldsymbol{r})+\sum_{r^{\prime}} J_{r-r^{\prime}}\left\langle\sigma_{r^{\prime}}\right\rangle
$$

Just as in the mean field approximation, also with this procedure all correlations between spins are neglected. Nevertheless, it is now possible to calculate in first approximation the influence of fluctuations on various thermodynamic quantities. This can most clearly be illustrated by calculating the specific heat in zero field:

$$
C=\frac{\mathrm{d}}{\mathrm{d} T}\langle\hat{H}\rangle=\frac{1}{2} \sum_{r, r^{\prime}} J_{r-r^{\prime}} \frac{\mathrm{d}}{\mathrm{d} T}\left\langle\sigma_{r} \sigma_{r^{\prime}}\right\rangle
$$

With the help of the random phase approximation, eq. (4.78), it is possible to calculate

$$
\frac{\mathrm{d}\left\langle\sigma_{r}\right\rangle}{\mathrm{d} B\left(\boldsymbol{r}^{\prime}\right)}=\frac{1}{T}\left\{\left\langle\sigma_{r} \sigma_{r^{\prime}}\right\rangle-\left\langle\sigma_{r}\right\rangle\left\langle\sigma_{\boldsymbol{r}^{\prime}}\right\rangle\right\}
$$


which allows to write the specific heat as

$$
C=C_{0}+C_{\text {corr }}
$$

where $C_{0}$ and $C_{\text {corr }}$ are given by:

$$
\begin{aligned}
& C_{0}=-\frac{1}{2} \sum_{r, r^{\prime}} J_{r-r^{\prime}} \frac{\mathrm{d}}{\mathrm{d} T}\left\langle\sigma_{r}\right\rangle\left\langle\sigma_{r^{\prime}}\right\rangle \\
& C_{\text {corr }}=-\left.\frac{1}{2} \sum_{r, r^{\prime}} J_{r-r^{\prime}} \frac{\mathrm{d}}{\mathrm{d} T} T \frac{\mathrm{d}\left\langle\sigma_{r}\right\rangle}{\mathrm{d} B\left(r^{\prime}\right)}\right|_{B=0} .
\end{aligned}
$$

As can be seen from eqs. (4.81c) and (4.80), $C_{\text {corr }}$ measures how much the spins fluctuate in a correlated way. If we limit ourselves to temperatures $T>T_{\mathrm{c}}$, where

$$
T_{\mathrm{c}}=J=\sum_{r-r^{\prime}} J_{r-r^{\prime}}
$$

one gets immediately from eq. (4.77) $\langle\sigma\rangle \equiv 0$, which leads in eq. (4.81b) to

$$
C_{0}(T) \equiv 0 \quad \text { for } T>T_{\mathrm{c}} .
$$

Just below $T_{c}$, one gets from an expansion of eq. (4.77) in terms of $\left(1-T / T_{c}\right)$ the well known finite jump $\Delta C_{0}$ in the specific heat, characteristic for the mean field theory. In this case, this gives

$$
\Delta C_{0}\left(T_{\mathrm{c}}\right)=\frac{3}{2} N
$$

The contribution $C_{\text {corr }}$ (for $T>T_{\mathrm{c}}$ ), due to the correlations, of the specific heat can be calculated from eqs. (4.78) and (4.81c) by a Fourier expansion of all quantities involved. One gets

$$
C_{\text {corr }}(T)=-\frac{1}{2} \sum_{q} J(q) \frac{\mathrm{d}}{\mathrm{d} T}\left(\frac{T}{T-J(q)}\right) \quad \text { for } T>T_{\mathrm{c}}
$$

where the summation with respect to $q$ goes over the first Brillouin zone, and

$$
J(q)=\sum_{\rho} J_{\boldsymbol{\rho}} \mathrm{e}^{-\mathrm{i} \boldsymbol{q} \cdot \boldsymbol{\rho}}
$$

In view of the application to superconductors, we choose the following form for the interaction:

$$
J_{r-r^{\prime}}= \begin{cases}J_{0} & \left|\boldsymbol{r}-\boldsymbol{r}^{\prime}\right| \leq \frac{1}{2} d \\ 0 & \left|\boldsymbol{r}-\boldsymbol{r}^{\prime}\right|>\frac{1}{2} d .\end{cases}
$$

This means that within a sphere of diameter $d, Z$ spins are coupled to the central spin; for a cubic 
lattice with lattice constant $a$, one gets for $Z$

$$
\frac{1}{6} \pi d^{3}=Z a^{3}
$$

For $T \geq T_{\mathrm{c}}$ and close to $T_{\mathrm{c}}$, one gets for the correlation contribution

$$
\frac{C_{\text {corr }}(T)}{\Delta C_{0}}=\frac{1}{Z} \frac{1.7}{\sqrt{\left(T-T_{\mathrm{c}}\right) / T_{\mathrm{c}}}}=\frac{1.7}{Z}|\varepsilon|^{-1 / 2}
$$

where $\varepsilon \equiv\left(T-T_{\mathrm{c}}\right) / T_{\mathrm{c}}$. The temperature region $\varepsilon_{\mathrm{c}}$, where fluctuations are important, can be estimated roughly by putting $\Delta C_{0}=C_{\text {corr }}$; this condition leads to $\varepsilon_{\mathrm{c}} \simeq 3 / Z^{2}$. As this result is independent of the specific structure of the Ising model, it should be true for every mean field theory. Applied to the BCS-theory of superconductivity, one has

$$
Z=\frac{4}{3} \pi n R_{0}^{3}
$$

where $\boldsymbol{R}_{\mathbf{0}}$ is the range of the attractive electron-electron interaction and $n$ is the density of particles which interact with each other.

For a superconductor with a short mean free path $l$, one has from eq. (4.3)

$$
R_{0} \simeq \sqrt{l \xi_{0}}
$$

while the Pippard coherence length $\xi_{0}$ is given by

$$
\xi_{0} \simeq 0.18 \hbar v_{\mathrm{F}} / k T_{\mathrm{c}} .
$$

The density of interacting particles is given by the electron density times the degeneracy $k T_{\mathrm{c}} / \varepsilon_{\mathrm{F}}$ :

$$
n \simeq \frac{p_{\mathrm{F}}^{3}}{3 \pi^{2} \hbar^{3}} \frac{k T_{\mathrm{c}}}{\varepsilon_{\mathrm{F}}}
$$

This gives as the temperature region where in a bulk superconductor fluctuations should be important:

$$
\varepsilon_{\mathrm{c}} \simeq \frac{\hbar^{4}}{\left(l_{\mathrm{F}}\right)^{3}\left(p_{\mathrm{F}} \xi_{0}\right)}
$$

Under the most favourable conditions, one can assume that $\left(l p_{\mathrm{F}}\right) / \hbar=10$, and $\left(p_{\mathrm{F}} \xi_{0}\right) / \hbar \simeq 10^{4}$, so that $\varepsilon_{\mathrm{c}} \simeq 10^{-7}$. Therefore, it is not surprising that the BCS mean field theory of superconductivity is in such good agreement with experiments performed on bulk superconductors.

In the case of a small superconducting particle, all dimensions are small compared with $R_{0}$ and the coherence length. Therefore, fluctuations cannot vary spatially, and only terms with $q=0$ can give a contribution in eq. (4.85). The specific heat of a small particle is then given by

$$
C_{\text {corr }}=\frac{1}{2}\left(J(0) T_{\mathrm{c}} /\left(T-T_{\mathrm{c}}\right)^{2}\right)=\frac{1}{2} \varepsilon^{-2} \text {. }
$$


Again defining the temperature region where fluctuations could be seen by putting $C_{\text {corr }}=\Delta C_{0}$, one gets

$$
\varepsilon_{\mathrm{c}} \simeq\left(1 / 3 d^{3} n\right)^{1 / 2} \quad\left(d \ll R_{0}\right),
$$

where $d$ is the dimension of the particle. For a typical superconductor with $d \simeq 10 \mathrm{~nm}$ one gets $\varepsilon_{\mathrm{c}} \simeq 10^{-1}$. This means that fluctuations should be visible clearly in small particles.

These rather general ideas have been elaborated by many workers [227]. It is obvious that in a superconductor there are other properties to be measured which are much more sensitive to fluctuations than the specific heat, like the resistivity; however, for a detailed theoretical analysis of these quantities, the random phase approximation has to be generalized from equilibrium to dynamic properties.

For equilibrium properties, Shmidt [231], Parkinson [232] and Mühlschlegel, Scalapino and Denton [124] have calculated the contribution of fluctuations to the thermodynamic quantities within the framework sketched here for the Ising model. All these authors start from the Ginzburg-Landau form of the free energy $F$, relative to the normal state, and for a zero-dimensional small superconductor of volume $V . F$ is then given by

$$
F=\left(a|\Delta|^{2}+\frac{1}{2} b|\Delta|^{4}\right) V
$$

where $a=a_{0} \varepsilon$ changes sign at $T_{\mathrm{c}} ; a_{0}$ and $b$ are positive constants approximately independent of temperature near $T_{\mathrm{c}}$ and which can be calculated from the microscopic BCS-theory, and $\Delta$ is the order parameter. $a$ and $b$ are related to the thermodynamical critical field $H_{c}(T)$ by

$$
\frac{1}{2} \mu_{0} H_{\mathrm{c}}^{2}(T)=a^{2} / 2 b
$$

(see also our discussion of the Ginzburg-Landau theory in section 4.2). According to the standard methods of statistical mechanics, the average value of the square of the order parameter is then given by

$$
\left\langle|\Delta|^{2}\right\rangle=\frac{\int|\Delta|^{2} \mathrm{e}^{-F / k T} \mathrm{~d}^{2} \Delta}{\int \mathrm{e}^{-F / k T} \mathrm{~d}^{2} \Delta}
$$

where $\mathrm{d}^{2} \Delta$ refers to the real and imaginary parts of the complex order parameter $\Delta=|\Delta| \mathrm{e}^{\mathrm{i} \phi}$. These integrals can be solved analytically $[124,231,232]$, and one obtains

$$
\left\langle|\Delta|^{2}\right\rangle=\left|\Delta_{0}\right|^{2}\left(1-\frac{1}{q} h(q)\right)
$$

$\left|\Delta_{0}\right|^{2}$ is the equilibrium value of the square of the order parameter, and is given from eq. (4.97) with $\delta F=0$ :

$$
\left|\Delta_{0}\right|^{2}=-a / b \sim|\varepsilon|
$$

(note that $a=a_{0} \varepsilon<0$, for $T<T_{\mathrm{c}}$ ), and 


$$
h(q)=\frac{\mathrm{e}^{-q^{2} / 2}}{\sqrt{2 \pi} \operatorname{erfc}(-q)}
$$

where $\operatorname{erfc}(x)$ denotes the normal probability function and $q=\left(a^{2} / b k T\right)^{1 / 2}$. In analogy with the condition $\Delta C_{0}=C_{\text {corr }}$ and following Skocpol and Tinkham [227], a critical temperature region can be defined where fluctuations should be important, by putting the fluctuation energy $k T_{c}$ equal to the free energy $\left|F_{0}\right|$

$$
\left|F_{0}\right|=k T_{\mathrm{c}}
$$

which leads to a critical region with a characteristic width

$$
\varepsilon_{\mathrm{c}}=\left(2 b k T_{\mathrm{c}} / a_{0}^{2} V\right)^{1 / 2}
$$

The first term in eq. (4.100) gives the usual equilibrium value of $|\Delta|^{2}$ obtained by minimizing $F$, while the second term describes the fluctuations. These die away exponentially below $T_{c}$, and roughly as $\left(T-T_{\mathrm{c}}\right)^{-1}$ above $T_{\mathrm{c}}$. Fig. 4.8 shows the mean-square magnitude of the order parameter as a function of the normalized temperature.

The most direct measure of $\left\langle|\Delta|^{2}\right\rangle$ is the diamagnetic susceptibility of a small particle. For a particle with a diameter $d$ smaller than the penetration depth and the coherence length, eq. (4.11) leads to a diamagnetic susceptibility given by

$$
\chi \simeq-\frac{1}{40} \frac{d^{2}}{\lambda^{2}} \quad\left(d \ll \lambda, \xi_{0}\right)
$$

Following the usual interpretation of $\left\langle|\Delta|^{2}\right\rangle$ as the average density of Cooper pairs $\left(|\Delta|^{2} \sim n_{\mathrm{S}}^{*}=\frac{1}{2} n_{\mathrm{S}}, n_{\mathrm{S}}\right.$

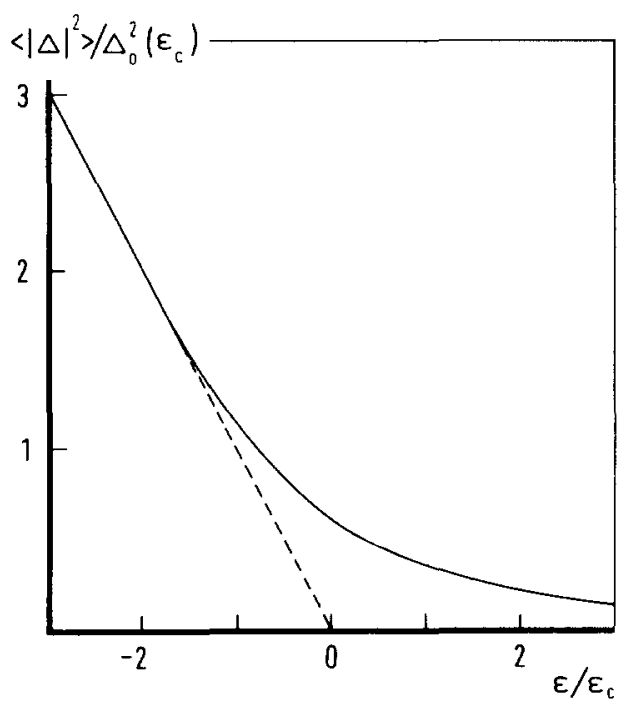

Fig. 4.8. Mean-square magnitude of the order parameter as a function of the normalized temperature for a small superconducting particle. $\Delta_{0}(|\varepsilon|)$ is the mean field value of the order parameter. The dashed line indicates this mean field behaviour, where fluctuations are neglected, it is approached far from $T_{\mathrm{c}}$. 
denoting the density of single electrons in the ground state), the London penetration depth is given by

$$
1 / \lambda^{2}=\left(\mu_{0} e^{* 2} / m^{*}\right)\left\langle|\Delta|^{2}\right\rangle
$$

where $e^{*}, m^{*}$ and $n_{\mathrm{S}}^{*}$ the charge, mass and density of the Cooper pairs. Therefore, the susceptibility is

$$
\chi \sim d^{2}\left\langle|\Delta|^{2}\right\rangle
$$

This relation has very carefully been tested experimentally by Buhrman and Halperin [233] on small single-crystal aluminium particles using a SQUID to measure the susceptibility. Fluctuation effects are observed in the critical region and they are, properly scaled and averaged over the particle size distribution, in excellent agreement with the theoretical predictions (fig. 4.9).

In a similar way, it is possible to calculate the specific heat in the superconducting state of zero-dimensional particles $[124,231,234-236]$. The specific heat is given by the thermodynamic relation

$$
C_{V}=-\frac{T}{V} \partial^{2}\langle F\rangle / \partial T^{2}
$$

where $\langle F\rangle$ is the weighted average of the free energy of eq. (4.97) over all values of the order parameter. This has been done in a very basic paper by Mühlschlegel, Scalapino and Denton [124]. The results of their calculations for the specific heat of small superconducting particles in the Ginzburg-Landau approach are shown in fig. 4.10. In addition, Mühlschlegel et al. extended the microscopic calculations by taking

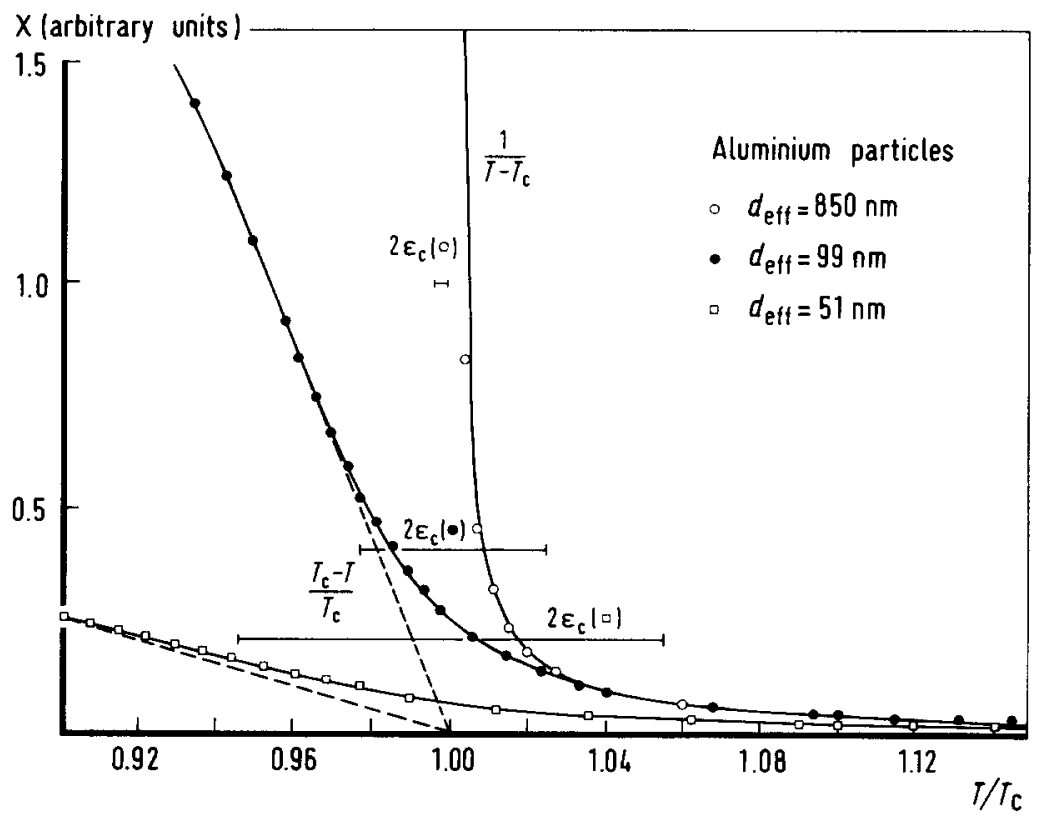

Fig. 4.9. Measured diamagnetic susceptibility of small superconducting aluminium particles with different mean particle size in the temperature region around $T_{\mathrm{c}}$. The solid lines represent the results of the Ginzburg-Landau theory including fluctuation effects and properly averaged over the measured particle size distribution. The dashed lines indicate the mean field behaviour below $T_{c}$. The region $2 \varepsilon_{c}(d)$, where fluctuations should be present, is indicated by a horizontal bar for the different particle diameters $d_{\text {eff }}$. (From Buhrman and Halperin [233].) 


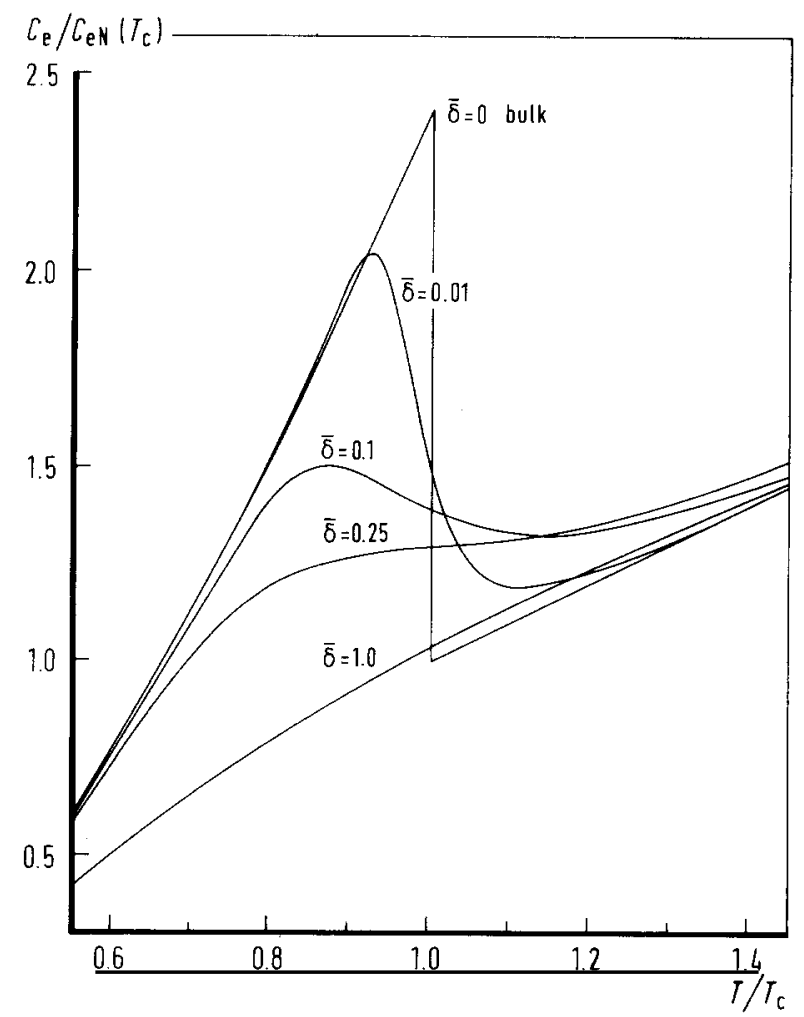

Fig. 4.10. Calculated temperature dependence of the total specific heat $C_{\mathrm{e}}$ of small superconducting particles normalized to the specific heat $C_{\mathrm{eN}}\left(T_{\mathrm{c}}\right)$ in the normal state at $T_{\mathrm{c}} \cdot \bar{\delta}=\delta / k T_{\mathrm{c}}=2 /\left(\rho\left(\varepsilon_{\mathrm{F}}\right) k T_{\mathrm{c}}\right.$ ). For a particle with $d \simeq 10 \mathrm{~nm}$, one has approximately $\bar{\delta} \simeq 1$. (From Mühlschlegel, Scalapino and Denton [124].)

into account the discrete structure of the one-electron spectrum in small particles. Using the equal level scheme as discussed in Appendix II, they employed the functional-averaging method explicitly using the BCS Hamiltonian under QSE conditions. As usual, the pairing interaction is taken between timereversed states of the electrons in the small particle. The relevant parameter of this sort of calculation is the ratio of the mean single-electron level spacing $\delta$ to $k T_{\mathrm{c}}$ :

$$
\bar{\delta}=\frac{\delta}{k T_{\mathrm{c}}}=\frac{2}{\rho\left(\varepsilon_{\mathrm{F}}\right) k T_{\mathrm{c}}}
$$

Note that this treatment of the QSE retains the influence of static fluctuations, because the full functional integration is performed, in contrast to the results discussed in section 4.3.3. Perhaps the most important small-particle effect is the restriction to fixed electron numbers as discussed in Kubo's treatment of the QSE in section 3.2.1; for superconducting particles, where electron pairing plays a major role, the restriction to fixed electron number may be even more important. Although some studies of this problem have been done in the essential work of Mühlschlegel, Scalapino and Denton [124] by projecting the canonical ensemble from the usual grand canonical ensemble by means of a saddle-point integration, this problem is still open for discussions.

In a set of beautiful experiments, Tsuboi and Suzuki [237, 238] have measured the electronic specific 
heat of fine particles of $\mathrm{Sn}$ with an average diameter ranging from 25 to $220 \mathrm{~nm}$ over a temperature range from $0.4 T_{c}$ to $1.5 T_{c}$, in zero magnetic field (fig. 4.11) and in fields up to $3 \mathrm{~T}$. An ensemble of $\mathrm{Sn}$ particles insulated from each other by oxide layers was prepared by depositing $\mathrm{Sn}$ islands in vacuum and then oxidizing their surfaces, and this procedure was performed repeatedly (see section 5.2.3). The Sn particles were deposited at room temperature, and the average particle size was controlled by using a well defined amount of $\mathrm{Sn}$ for one shot of evaporation. Between 20 and 200 layers were used for the different samples, and the weight of the Sn particles was of the order of $1 \mathrm{mg}$. The specific heat was measured by using an ac-temperature calorimetry technique, the contribution of the Sn particles to the total heat capacity of the arrangement was of the order of $10 \%$. As can be seen in fig. 4.11 , the

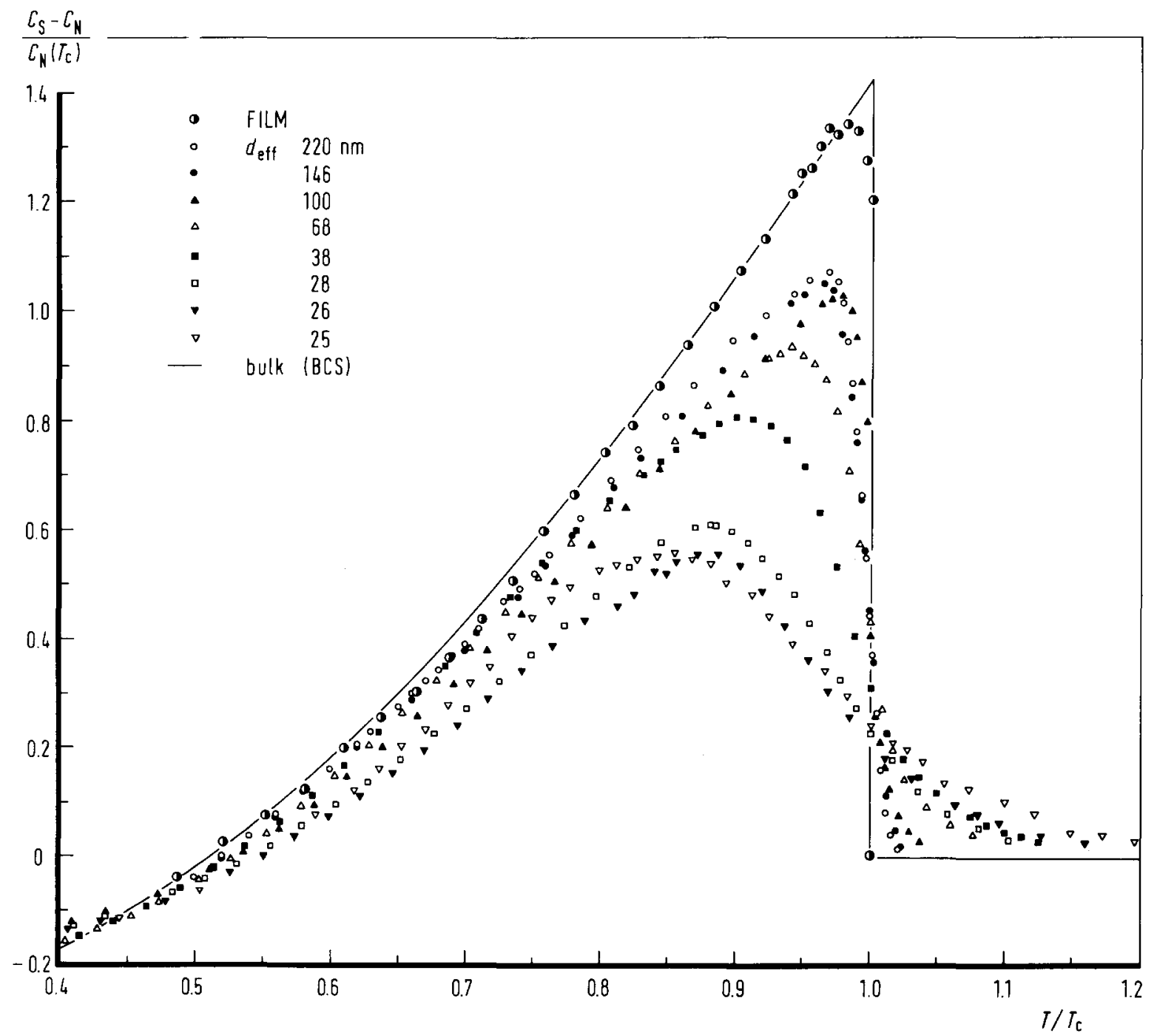

Fig. 4.11. Measured normalized difference $\left(C_{\mathrm{S}}-C_{\mathrm{N}}\right) / C_{\mathrm{N}}\left(T_{\mathrm{c}}\right)$ of the specific heat in the superconductive and normal state respectively, for tin particles with different diameters, as a function of the reduced temperature. The measurements are normalized to $C_{\mathrm{N}}\left(T_{\mathrm{c}}\right)=\gamma T_{\mathrm{c}}$, with $\gamma=$ $1.78 \times 10^{-3} \mathrm{JK}^{-2} \mathrm{~mol}^{-1}$. The ensemble of tin particles, isolated from each other by oxide layers, was prepared by depositing tin islands in vacuum and then oxidizing their surfaces, repeatedly. (From Tsuboi and Suzuki [237].) 
measurements of the "bulk" material (i.e. a Sn film prepared in the same way as the particles) are in good agreement with the BCS results, documenting the reliability of the experimental method. As the particle size decreases, the broadening of the transition into the superconducting state is enhanced, the peak shifts towards lower temperature and decreases in height. These effects show clearly the existence of a wide critical region of temperature and are in striking, at least qualitative, agreement with the theoretical predictions of Mühlschlegel, Scalapino and Denton [124] (fig. (4.10)). The broadening of the transition into the superconducting state as measured in magnetic fields [238] is explained by Suzuki and Tsuboi by assuming that the thermodynamical fluctuations depend on the magnetic field only through the change of $T_{\mathrm{c}}(H)$, at least for low fields; for increasing fields, the particle size distribution becomes the dominant factor determining the broadening. The temperature and size dependence of the critical field seems to be in good agreement with the theory as discussed in section 4.3.2.

Results similar to the ones of Tsuboi and Suzuki [237] have recently been reported by Filler, Lindenfeld, Worthington and Deutscher [239] on the heat capacity of granular aluminium films. Granular aluminium consists of metallic aluminium grains with a particle size of the order of $d \simeq 3 \mathrm{~nm}$, embedded in a matrix of aluminium oxide. By changing the deposition conditions, Filler et al. were able to produce films where the normal state resistivity changed over three orders of magnitude. They interpret this increase in resistivity as the result of the presence of an increasing amount of oxide between the grains, and therefore the grains become progressively decoupled until they are effectively isolated. These experiments show that as the resistivity increases, bulk superconductivity as described by the transition in the heat capacity disappears gradually, again in good qualitative agreement with the predictions of Mühlschlegel, Scalapino and Denton [124].

All these specific heat experiments support the theoretical considerations on fluctuations in small superconducting particles. The fact that in these experiments almost no transition in the heat capacity remains for the smallest particles might indicate that the grains are no longer able to remain superconducting, at least not in the thermodynamic BCS sense. This could then be interpreted as an experimental indication that superconductivity ceases to exist if the particles are so small that $d \leq d_{\mathrm{A}}$ (see section 4.3.3). However, the behaviour of these particle-film systems in the superconducting state might be influenced by the change in the mutual coupling between the particles (percolation type of behaviour [240]) or the change due to the oxide layer present. The many complications due to the influence of the change in the lattice vibrations or the surface vibrations [241-243], as well as the influence of oxide overlayers on superconductivity should be understood and analysed carefully.

\section{Preparation of small particles}

In this section we will present a discussion of a number of techniques used to prepare samples containing small metallic particles. Small particle systems include colloidal suspensions of metals (section 5.1), discontinuous thin films (section 5.2), gas-evaporated particles and cluster beams (section 5.3), colloidal particles contained in photosensitive glasses or other types of matrices (section 5.4), and metal dispersions forced into the cavities of porous materials (section 5.5). We do not discuss the purely chemical methods to produce well defined "molecular clusters" of which a large number has been described in recent years $[244,245]$.

From an experimental point of view, the small particles must satisfy severe requirements in order to give meaningful results. Their sizes must be very small and well defined: Depending on the type of experiment and the characteristic lengths involved sizes down to $1 \mathrm{~nm}$ are required, while the size 
distribution must be as narrow as possible. In many experiments, a deconvolution of the data is possible when the size distribution is known; for other experiments, the size distribution must be extremely narrow: e.g. in section 3.5.3 it was shown that the oscillations in the far infrared absorption spectrum expected for the symplectic ensemble are easily wiped out by a small size distribution of the particles in the sample. Moreover, poor chemical purity of either particles, their surfaces, or the supporting matrix will strongly influence the relaxation times in magnetic resonance experiments, or lead to spurious signals in measurements of the magnetic susceptibility (e.g. see section 3.5.5).

\subsection{Metal colloids}

A very simple system for the study of quantum size effects is a suspension of submicron size colloidal particles. Chemists have devoted a large amount of work to the study of colloidal systems, and well defined prescriptions are available for quite a lot of materials: A reducing agent added to a solution of metal ions causes condensation in the newly formed supersaturated solution of metal atoms. By a proper choice of concentration, temperature and reducing agent it is, in some cases, possible to produce submicron size particles of well defined dimensions. Many hydrosols are stable as a result of the action of an electric double layer of ions surrounding the particles, and when protective agents, such as gelatin, are added, the suspensions are even stable on dehydration.

In 1964 Doremus [21] studied the optical properties of gold hydrosols; soon thereafter Marshall and Wilenzick [246] published results of the determination of the Mössbauer recoil free fraction for gamma ray absorption of colloidal particles of gold. Superparamagnetic properties were studied using the Mössbauer effect in samples prepared from hydrosols of iron, nickel, cobalt, or their compounds. Svedberg's method [247] was used by Tanner, Sievers and Buhrman [158] to produce colloidal dispersions of lead and copper particles in acetone; in this case, the particles have been formed by striking high frequency arcs between metal chips, under a polar liquid. This method can be used for many materials, but the size distribution obtained is usually quite poor.

\subsubsection{Noble metal hydrosols}

The colloidal systems which have been most extensively studied are hydrosols of the noble metals. Turkevich, Stevenson and Hillier [248] prepared gold hydrosols with different chemical reagents and under different conditions. They succeeded in growing colloidal gold particles with a comparatively narrow size distribution using the following recipe: Prepare $950 \mathrm{ml}$ of a chlorauric acid solution containing $100 \mathrm{mg}$ gold; heat this solution to $80^{\circ} \mathrm{C}$ and add, instantaneously and under vigorous stirring, $50 \mathrm{ml}$ water containing $1 \%$ by weight sodium citrate. After five to ten minutes the solution will get a bluish grey colour that turns into red. After an hour the solution is dark ruby-red and the reaction is completed. The hydrosol may then be stabilized by adding a protective agent, as e.g. $250 \mathrm{mg}$ gelatin. A typical size distribution for colloidal gold particles obtained in this way is given in fig. 5.1a; the mean particle size is $\bar{d}=14.7 \pm 1.3 \mathrm{~nm}$. Turkevich and coworkers found that the spread in particle size is connected with a spread in time of the formation of nuclei. The rate of formation of the nuclei depends on the concentration; the nucleation process seems to be inhibited for a concentration above a certain critical concentration of nuclei. Then only the particle growth process will continue until, by lack of gold ions, the reaction comes to an end. In fig. 5.1b the size histogram is given for a solution with only $33 \%$ of the concentrations given above. The mean particle size increased to $\bar{d}=19.1 \pm 2.1 \mathrm{~nm}$ in this case. Fig. 5.2 shows the dramatic effects of heterogeneous nucleation on the width of the size distribution; the reducing agent was added slowly to the chlorauric acid solution, drop by drop: The nucleation process is 

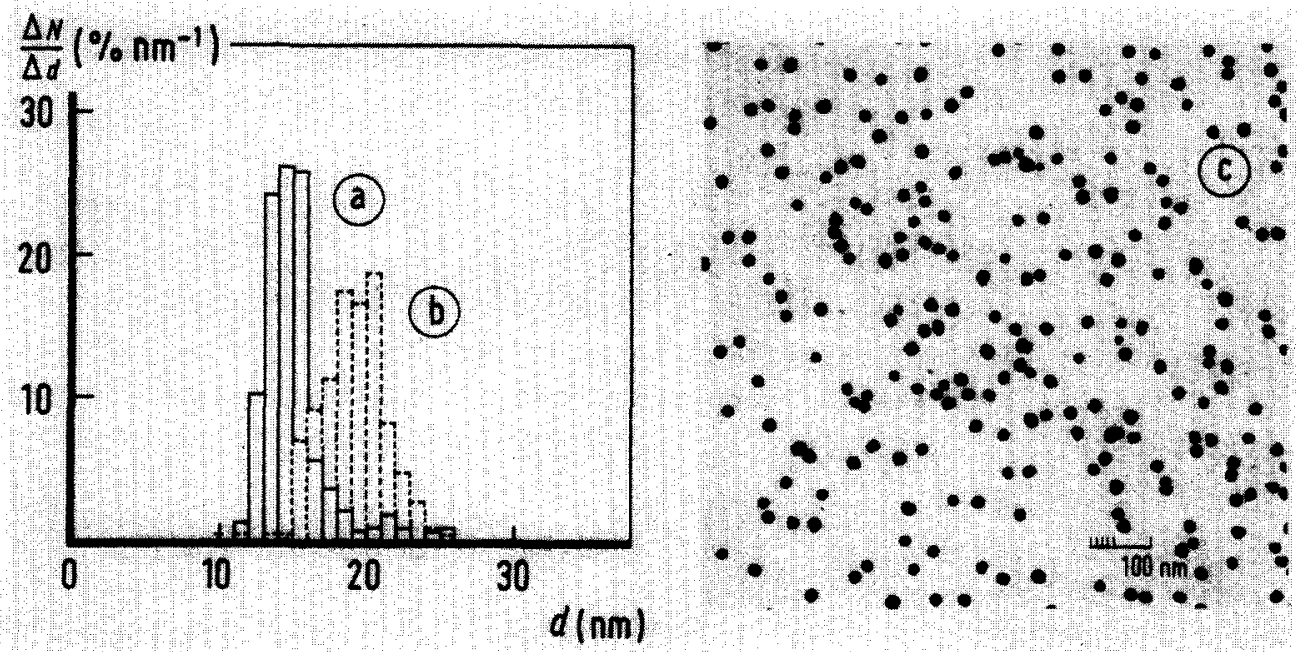

Fig. 5.1. Size distributions for colloidal gold particles. (a) For a hydrosol prepared from a chlorauric acid solution with sodium citrate; $\bar{d}=14.7 \pm 1.3 \mathrm{~nm}$. (b) For a hydrosol prepared as in (a), but the chemical reagents were more diluted; $\bar{d}=19.1 \pm 2.1 \mathrm{~nm}$. (c) Electron microscope micrograph corresponding to the size histogram (b).

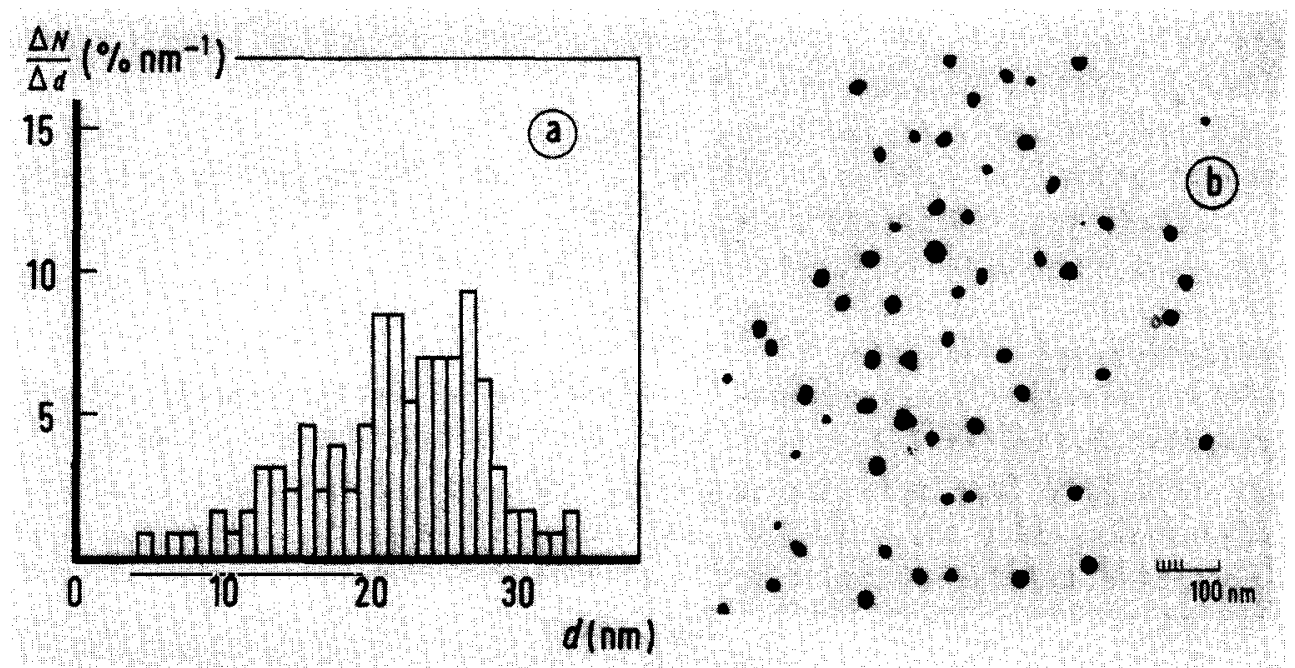

Fig. 5.2. (a) A size distribution of colloidal gold particles showing clear effects of heterogeneous nucleation: $\bar{d}=21.5 \pm 6 \mathrm{~nm}$. (b) Electron microscope micrograph of the colloidal particles.

not inhibited, and both nucleation and particle growth take place simultaneously. Therefore, the length of the aging time of the individual particles is not very well determined.

Zsigmondy [162] showed that larger particles may be obtained by using a seeding technique: A monodisperse hydrosol prepared previously is added to a solution containing the metal ions, and then a reducing agent is added. Colloidal gold particles of average diameter up to $100 \mathrm{~nm}$ were prepared in this way without degradation of the relative size distribution [248]. 
Smaller particles may be formed when the gold solution is reduced by using phosphorus. Particles with a mean diameter $\bar{d}$ of $3.4 \mathrm{~nm}$ and a size distribution as shown in fig. 5.3a were obtained as follows: $350 \mathrm{mg} \mathrm{HAuCl} \mathrm{H}_{4}$ was solved in $1000 \mathrm{ml}$ water and the solution was neutralized using $1 \mathrm{~N} \mathrm{~K}_{2} \mathrm{CO}_{3} ; 10 \mathrm{ml}$ of a saturated solution of phosphorus in diethylether was then added at room temperature under vigorous stirring. A suspension of phosphorus particles is created, and on the surface of the phosphorus particles small gold clusters are formed which act as nuclei for the growth of the colloidal gold particles. After two hours, the reaction is completed, the hydrosol can then be stabilized with $250 \mathrm{mg}$ gelatin solved in water; to remove all traces of phosphorus, it is necessary to boil the solution for several hours, preferably while passing through it a flow of filtered air.
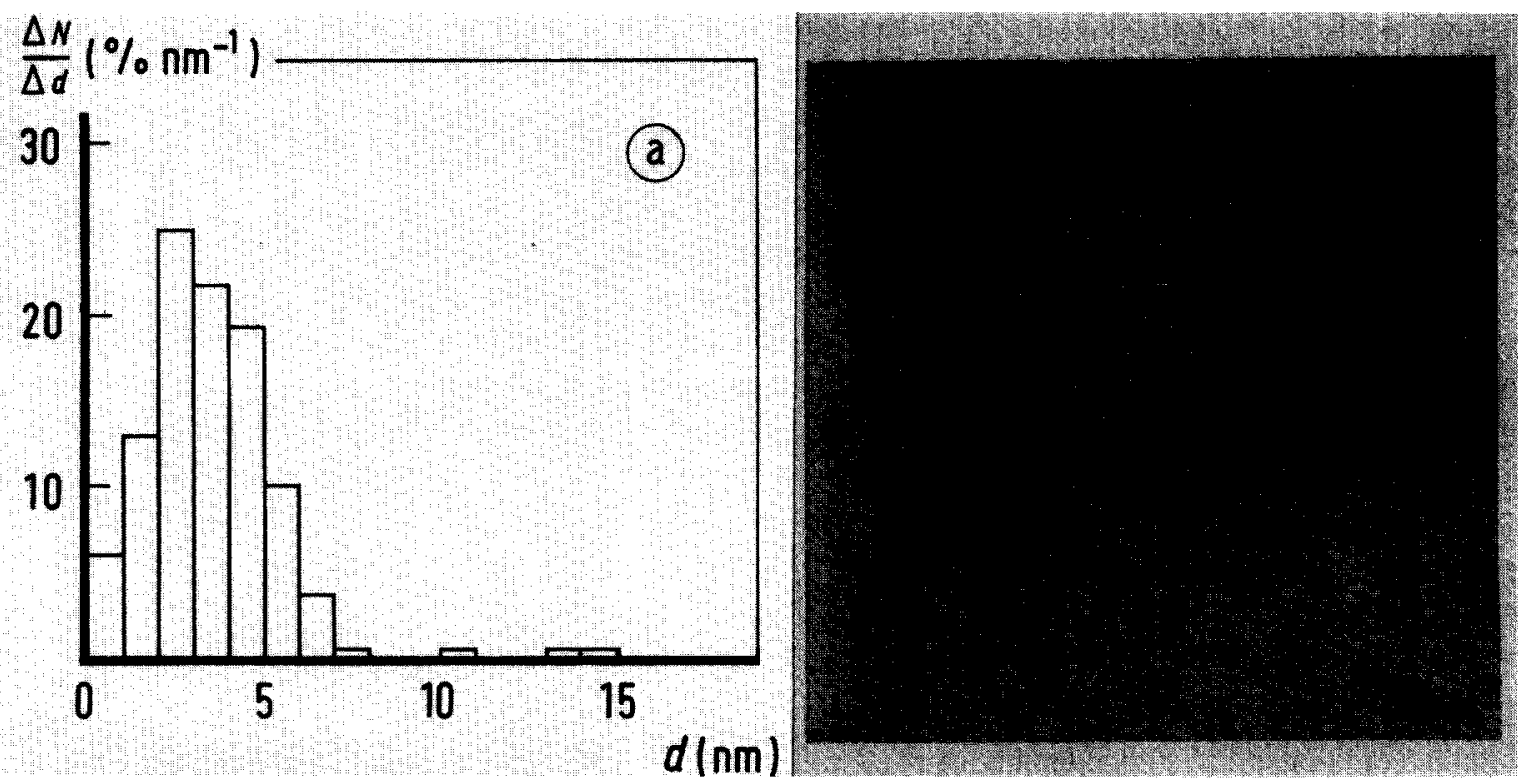

Fig. 5.3. (a) Size distribution for colloidal gold particles prepared from an aqueous chlorauric acid solution with a saturated solution of phosphorus in diethylether; $\bar{d}=3.4 \pm 1.6 \mathrm{~nm}$. (b) Electron microscope micrograph of the colloidal gold particles.

Ion exchange and dialysis can be applied to clean the samples prior to dehydration, and it was found that these techniques did not influence the size distribution of the hydrosols in a noticeable way.

The preparation of platinum colloids was reviewed by Wilenzick et al. [249], and recently, Marzke and coworkers $[157,172]$ used these techniques for a study of the magnetic properties of platinum particles with a mean diameter of $2 \mathrm{~nm}$.

\subsubsection{Mercury colloids}

Shoenberg [202] and Whitehead [203] studied the magnetic properties of superconducting mercury particles. The colloidal suspension of mercury was obtained by adding a solution of mercurous nitrate to a solution containing a protective agent prepared from egg albumen; the reducing agent was hydrazine. The solution was then acidified with acetic acid and the protective agent coagulated and precipitated, taking down all the mercury particles. Aging at room temperature caused an increase of the average diameter, probably due to coagulation.

Meier [250] followed a recipe of Feick [251]: $0.5 \mathrm{ml}$ of $0.5 \mathrm{~N} \mathrm{Hg}_{2}\left(\mathrm{NO}_{3}\right)_{2}$ was diluted with $100 \mathrm{ml}$ of a 
$0.1 \%$ gelatine solution and this solution was slightly acidified; $0.1 \mathrm{~N} \mathrm{Na}_{2} \mathrm{~S}_{2} \mathrm{O}_{4}$ was added as reducing agent and, depending on the amount of reagents used, samples with an average particle diameter well below $10 \mathrm{~nm}$ could be prepared. It was observed that the mean particle size of the colloids decreased in the course of time, in accordance with Feick's observation that the hydrosols were less stable for bigger particles.

\subsection{Granular metal films}

The most versatile way of producing samples containing small metallic particles is doubtless the method of thermal evaporation. In this section we will discuss some techniques to prepare thin layers of finely dispersed metal particles.

\subsubsection{Discontinuous thin films}

It is well known that in the first stages of growth of vacuum deposited films many metals tend to form island-like structures. As early as 1966 Doremus [23] used thin discontinuous films of gold, evaporated onto glass, for a study of the optical properties of small gold particles. Later Dupree, Forwood and Smith [151], and Monot, Châtelain and Borel [152] made attempts to observe conduction electron spin resonance in gold particles prepared by evaporation of a thin gold film onto sodiumchloride and amorphous quartz respectively.

From the point of view of nucleation theories [252], the physical processes involved in the formation of metal clusters in the initial stages of thin film growth have attracted much attention. Here, we take the work of Schmeisser [253] as a starting point for the introduction of some of the concepts used.

Schmeisser studied the process of the formation of gold clusters by evaporation of gold onto (100) cleavage planes of a $\mathrm{NaCl}$ single crystal in an ultrahigh vacuum. Substrate temperature, evaporation rate and deposition time were accurately controlled, and these parameters were varied over a wide range. The freshly deposited gold clusters were stabilized by covering them with a carbon film. The substrate was then dissolved in water and the sample was studied under an electron microscope. Measurements of the size and density of the clusters were made using a Quantimet 720 image analysing computer so that a considerable amount of information could be handled in an efficient way. In fig. 5.4

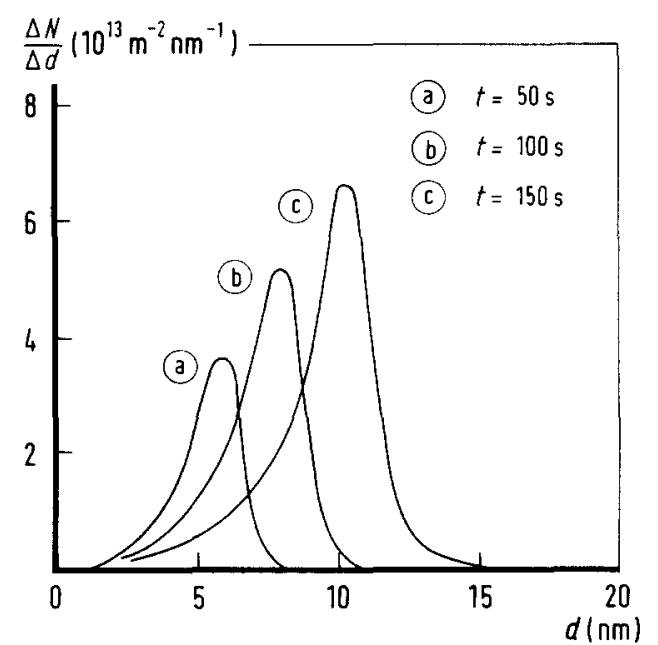

Fig. 5.4. The size distribution of gold clusters deposited in ultrahigh vacuum onto (100) cleavage planes of a $\mathrm{NaCl}$ single crystal, for increasing deposition times $t$. (From Schmeisser [253].) 
typical size distributions are presented. These results were obtained with a substrate temperature of $600 \mathrm{~K}$, an impingement rate $R=4.2 \times 10^{17} \mathrm{~m}^{-2} \mathrm{~s}^{-1}$, and deposition times of $50 \mathrm{~s}, 100 \mathrm{~s}$ and $150 \mathrm{~s}$. Under these conditions, the nucleation rate was $1.8 \times 10^{12} \mathrm{~m}^{-2} \mathrm{~s}^{-1}$.

\subsubsection{A model for island growth in thin films}

According to Schmeisser [253], the process of island growth may be described as follows: The gold atoms arriving at the substrate will be accommodated instantaneously, but the mean time $\tau_{\mathrm{e}}$ that such a single atom will remain on the substrate before reevaporation is determined by the binding forces as

$$
\tau_{\mathrm{e}}=\tau_{0} \exp \left(E_{\mathrm{b}} / k T\right)
$$

Here, $\tau_{0}$ is a characteristic time related to the vibrational spectrum of the substrate material and expected to be of the order of the reciprocal Debye frequency (in the case of $\mathrm{NaCl}: 1.7 \times 10^{-13} \mathrm{~s}$ ); $E_{\mathrm{b}}$ is the energy of binding to the surface; $k$ and $T$ are the Boltzmann constant and the temperature, as usual. In general, the threshold energy for changing sites on the substrate $E_{\mathrm{d}}$ is much smaller than the energy of binding $E_{\mathrm{b}}$. Therefore, the single atom will move over the surface with a characteristic time $\tau_{\mathrm{d}}$ before it eventually will reevaporate; $\tau_{d}$ is then given by

$$
\tau_{\mathrm{d}}=\tau_{0} \exp \left(E_{\mathrm{d}} / k T\right)
$$

Atoms migrating over the surface may join an already existing cluster and therefore contribute to its growth. If we consider the migration as a random walk problem and pass over to the equivalent diffusion equation, we find for the diffusive flux of atoms into a cluster of radius $r$ :

$$
\frac{\mathrm{d} N}{\mathrm{~d} t}=2 \pi r D \frac{N_{1}}{\sqrt{D \tau_{\mathrm{e}}}} \frac{K_{1}\left(r / \sqrt{D \tau_{\mathrm{e}}}\right)}{K_{0}\left(r / \sqrt{D \tau_{\mathrm{e}}}\right)}
$$

Here $N$ is the number of atoms contained in the cluster, the cluster is assumed to be a hemisphere of radius $r ; K_{0}$ and $K_{1}$ are modified Bessel functions of the zeroth and first order; $N_{1}$ is the concentration of single atoms at the surface, and $D$ is the diffusion constant, given by

$$
D=\frac{a_{0}^{2}}{4 \tau_{\mathrm{d}}}=\frac{a_{0}^{2}}{4 \tau_{0}} \exp \left(-E_{\mathrm{d}} / k T\right)
$$

where $a_{0}$ is the distance between neighbouring sites. By introducing the effective capture number $\sigma(r)$ as

$$
\sigma(r)=2 \pi \frac{r}{\sqrt{D \tau_{e}}} \frac{K_{1}\left(r / \sqrt{D \tau_{e}}\right)}{K_{0}\left(r / \sqrt{D \tau_{e}}\right)}
$$

eq. (5.3) can be rewritten as

$$
\left.\frac{\mathrm{d} N}{\mathrm{~d} t}\right|_{\text {diffusion }}=\sigma(r) D N_{1} .
$$


The differential equation for the number of single atoms present on the surface is now:

$$
\mathrm{d} N_{1} / \mathrm{d} t=R-N_{1} / \tau_{\mathrm{e}}-\sigma D N_{\mathrm{c}} N_{1}
$$

The first term on the right hand side is the rate of arrival of new atoms to the surface, the second term gives the loss caused by reevaporation, and the third term (containing the density of clusters $N_{\mathrm{c}}$ already formed) takes into account the diffusion of single atoms into these clusters. The value of the ratio $\left(\sigma D N_{c}\right) /\left(1 / \tau_{\mathrm{e}}\right)$ is at most $10^{-2}$ to $10^{-1}$ for the range of size and coverage in the experiment of Schmeisser, so that the solution of this differential equation is given with sufficient accuracy by

$$
N_{1}=R \tau_{e}\left(1-\exp \left(-t / \tau_{\mathrm{e}}\right)\right)
$$

As $\tau_{\mathrm{e}}$ is typically as small as $10^{-11} \mathrm{~s}$, eq. (5.8) reduces to $N_{1}=R \tau_{\mathrm{e}}$.

The increase of the number of atoms contained in a hemispherical cluster can be written as

$$
\frac{\mathrm{d} N(r)}{\mathrm{d} t}=\frac{1}{V_{1}} \frac{\mathrm{d} V}{\mathrm{~d} t}=\frac{2 \pi r^{2}}{V_{1}} \frac{\mathrm{d} r}{\mathrm{~d} t}
$$

where $V_{1}$ is the volume of one single atom. The growth of a cluster is caused by addition of atoms by surface diffusion, as described by eq. (5.6), but also by capture from the vapour phase by direct impingement

$$
\mathrm{d} N(r) / \mathrm{d} t=\sigma(r) D N_{1}+\pi r^{2} R .
$$

From eqs. (5.9) and (5.10), and using the approximation leading to eq. (5.8), one obtains

$$
\frac{R V_{1}}{2 \pi \sqrt{D \tau_{e}}}=\frac{1}{\left(D \tau_{\mathrm{e}}\right)^{3 / 2} \sigma(r)+\pi r^{2} / D \tau_{\mathrm{e}}} \frac{r^{2} t}{\mathrm{~d} t}
$$

or, in integral form, and with the substitution $x=r^{\prime} / \sqrt{D \tau_{\mathrm{e}}}$ :

$$
\int_{0}^{\pi / \sqrt{D \tau_{e}}} \frac{x^{2}}{\sigma(x)+\pi x^{2}} \mathrm{~d} x=\frac{R V_{1}}{2 \pi \sqrt{D \tau_{\mathrm{e}}}}
$$

For the range of size considered $\left(0<r / \sqrt{D \tau_{\mathrm{e}}} \leq 1\right)$, this integral can be approximated by

$$
\int_{0}^{r / 2 \overline{D \tau_{e}}} \frac{x^{2}}{\sigma(x)+\pi x^{2}} \mathrm{~d} x=0.044 \frac{r^{2}}{D \tau_{\mathrm{e}}} .
$$

$D \tau_{\mathrm{e}}$ can be found from eqs. (5.1) and (5.4), so that, finally, one obtains

$$
r^{2}=\frac{a_{0} V_{\mathrm{t}} R t}{0.176} \exp \left(\frac{E_{\mathrm{b}}-E_{\mathrm{d}}}{2 k T}\right)
$$


Schmeisser has found complete qualitative agreement for the maximum of the cluster sizes observed as compared with the expression given in eq. (5.13). The parameters used were: $E_{\mathrm{b}}-E_{\mathrm{d}}=0.38 \pm 0.06 \mathrm{eV}$, $V_{1}=17 \times 10^{-30} \mathrm{~m}^{3}$, and $a_{0}=0.4 \mathrm{~nm}$. $R$ was varied from $0.45 \times 10^{17}$ to $53 \times 10^{17} \mathrm{~m}^{-2} \mathrm{~s}^{-1}$, and the temperature of the substrate was varied from 543 to $723 \mathrm{~K}$.

According to eq. (5.13), the particle diameter increases proportional to the square root of the time, and this would lead to a size distribution which is proportional to the diameter, and for a constant nucleation rate, the small size tails of the size distribution curves should fall together. From the size distributions in fig. 5.4 we must therefore conclude that the concentration of very small clusters decreases systematically for extended deposition time. The third term in eq. (5.7) is too small to account for the observed discrepancy. Schmeisser showed that coalescence of clusters is possible, as a result of the mobility of the smaller clusters; then, the migration of clusters as a whole leads to a decrease of the concentration of the fastest clusters. Only after very long deposition times, the size distributions developed a tail extending towards the large particle sizes, indicating that coalescence had become important. In that case, the log-normal size distribution is appropriate [254].

Eq. (5.13) also indicates that the maximum cluster size is determined by the total amount of metal deposited $R t$. In addition, the particle size may be influenced by changing the substrate temperature: At higher temperature, but fixed evaporation rate, the nucleation rate decreases so that less particles are

produced; also, because of the temperature dependent factor in eq. (5.13), the average diameter decreases and the overall efficiency of the metal deposition is drastically reduced. Increase of the evaporation rate at fixed substrate temperature causes a strong enhancement of the nucleation rate. In the range of experimental conditions in Schmeisser's study, no saturation was observed of the maximum particle size below the size determined by eq. (5.13); it must therefore be concluded that the increase of the number of particles is compensated by a reduction of the loss of atoms through reevaporation. For some combinations of metal and substrate, critical deposition rates are found (dependent on the temperature) below which no nucleation is possible, and below which no film growth is observed. This is known to occur for instance with cadmium, zinc, mercury and aluminium [255].

\subsubsection{Preparation of samples}

The filling factor that can be achieved with granular metal films is obviously relatively low, as a result of its two-dimensional character. Monot et al. [152] reported that they were able to store $45 \mathrm{~cm}^{2}$ of sample in the cavity of the electron spin resonance spectrometer; this corresponded typically to $2 \times 10^{13}$ particles, well in excess of the limit of sensitivity of $10^{12}$ spins. Dupree and coworkers [151] tried to improve their signal to noise ratio by painting thin films of Durofix onto their samples and dissolving the $\mathrm{NaCl}$ substrate in water. Hammond and Kelly [123] used a very thin mylar foil as a substrate, and small particles were deposited by flash evaporation. Samples for NMR measurements were obtained by stacking on top of each other several precut pieces of the metal covered mylar foil.

An even better particle/substrate ratio can be achieved with the technique used by Hines and Knight [122]: Mylar foil was used again, but the metal particles were produced by flash evaporation of a fixed amount of metal after which the particles were covered with a layer of siliconmonoxide; by repeated evaporation of metal, alternated with deposition of $\mathrm{SiO}$, samples were prepared containing 60 layers of tin particles, and samples containing up to 130 layers of lead particles. More recently, this technique was used by Yee and Knight [134] to prepare copper particles. They deposited 400 layers of copper particles, separated by siliconmonoxide, on a mylar substrate; the mylar foil was finally folded over and over into a small package of a few mm diameter.

Zeller and Kuse [256] made samples consisting of several layers of flash evaporated tin particles 
which were isolated with a layer of $\mathrm{MgF}_{2}$, and Tsuboi [257] made insulated layers, merely by oxidizing the particles before the start of the next evaporation. Recently, Bezotosnyi and coworkers reported the use of lasers for the evaporation of metals [258]; with a laser pulse, very small amounts of metal can be evaporated very quickly and in a well controlled way. In this way particles could be obtained which are significantly smaller than the ones obtained with the conventional thermal evaporation techniques.

\subsubsection{Granular metal films}

Quite a different technique for producing films with finely dispersed particles was used in the early investigations of granular superconductors. Metal films evaporated onto helium cooled substrates showed a granular structure, but these thin films were unstable at higher temperatures. Cohen and Abeles [259] have made stable granular aluminium films by evaporation of aluminium at a rate of $10 \mathrm{~nm} / \mathrm{s}$ in a low pressure background of oxygen $\left(10^{-6}\right.$ to $5 \times 10^{-5}$ Torr $\left.\mathrm{O}_{2}\right)$ onto substrates which were both held at room temperature or cooled to nitrogen temperature. The presence of metal oxides seems to inhibit the formation of larger grains in the deposited films and to prevent coalescence. Deutscher et al. [260] observed a minimum of $3 \mathrm{~nm}$ for the average diameter of the grains with a substrate at room temperature, and of $2 \mathrm{~nm}$ with nitrogen cooled substrates. Under the latter conditions, the grain size was not very stable. Tin grains with a diameter smaller than $7 \mathrm{~nm}$ were found when tin was evaporated in $10^{-4}$ Torr oxygen onto a substrate cooled to $85 \mathrm{~K}$ [261].

Abeles and coworkers [262] prepared granular films of a number of metals with the method of cosputtering of metals and insulators. With this method, the volume fraction of metal can be varied over a wide range. Three regimes may be distinguished in these systems: In the metallic regime, the metal grains touch each other and form a continuum with dielectric inclusions; in the transition regime, the dielectric inclusions become interconnected and form a maze structure; and when the metal volume fraction is reduced below the matrix inversion fraction ( $40 \%$ for gold and platinum, $60 \%$ for nickel), the granular film is in the dielectric regime, where isolated metal particles are dispersed in a dielectric continuum. This last regime is well suited for numerous experiments on small particles. Typical average particle sizes are shown in fig. 5.5. It was found that the particle size was insensitive to the choice of metal or dielectric, and that it depended only on the volume fraction of metal. Moreover, the density of

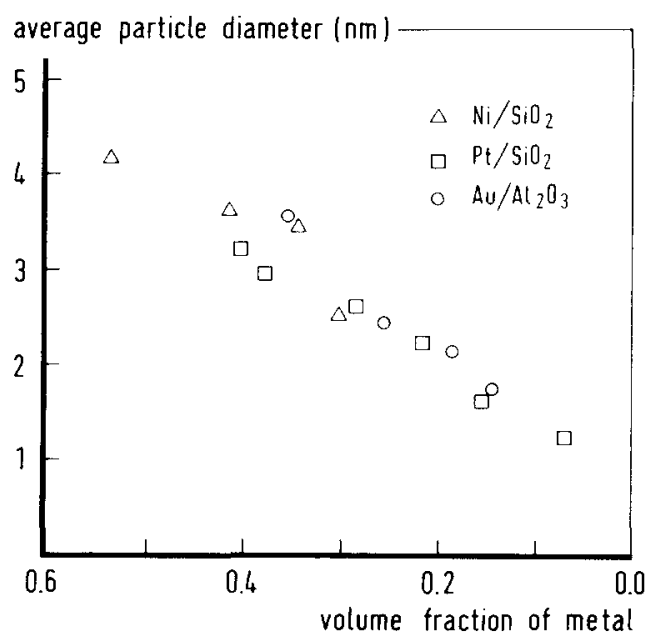

Fig. 5.5. The average particle diameter versus the volume fraction of metal, for a number of granular materials prepared by cosputtering of metal and dielectric. (From Abeles, Ping Sheng, Coutts and Arie [262].) 
particles that can be obtained in such granular films is much higher than in the case of thin films with an island like structure as discussed in section 5.2.1.

\subsection{Particles prepared by the gas-evaporation technique}

The method of producing finely divided particles by evaporation into a background gas dates back to the early 1930's when Pfund made the now famous and well known metallic blacks of a series of metals [263]: Fine particles of gold, silver, copper, nickel, zinc, lead, bismuth, antimony, selenium and tellurium were formed upon evaporation of these metals into air under a pressure of the order of 1 Torr. At higher pressures of the background gas and at higher evaporation rates, coarser particles were obtained. For these samples, the usual shiny metallic appearance is lost and the particles have a very dark appearance. This is a result of the absorption in the visible range of the spectrum, due to the very wide resonance appearing in particles and inhomogeneous materials with relevant dimensions smaller than the wavelength of the radiation, as is discussed in section 2 . Because of this selective absorption of visible radiation, surfaces covered with this sort of particles can be used as effective absorbers for solar radiation [64-66] in applications for photothermal conversion of solar energy.

\subsubsection{Evaporation in inert gas}

Kubo's paper of 1962 [3] has given a big impetus to the preparation and experimental study of small metallic particles. Kimoto, Kamiya, Nonoyama and Uyeda [264], and Kimoto and Nishida [265] have prepared microcrystals of up to twenty different metals. In a conventional vacuum evaporator purified argon gas was admitted to a pressure in the range from 0.5 to 50 Torr and then a small quantity of metal was evaporated. A cloud of smoke particles was formed, and it was drawn upwards by the convection of the gas, which was heated by the evaporation source. The particles were trapped on copper grids and studied with electron microscopic techniques.

Wada [266] noted that when the metals were evaporated into helium gas, the particles were almost ten times smaller than with evaporation into argon under otherwise the same conditions; when they were evaporated into xenon gas, they grew three to four times bigger in size. Similar results have been reported before by Gen, Ziskin and Petrov [267].

Yatsuya et al. [268,269] made a careful study of the processes that take place when aluminium is evaporated into a background of helium gas. A cloud of smoke particles developed when the evaporation boat filled with metal was heated, and its appearance depended both on the evaporation temperature and on the pressure of the background gas. They distinguished several regions in which different sizes and forms of crystallites were collected: Inner zone, inner front, and outer zone. The particles collected in the inner zone were nearly uniform in size; the size increased with increasing distance from the evaporation source until the particle size saturated. At a higher pressure of the inert gas, the saturation of the particle size was found at a smaller distance from the vapour source, and the mean particle size was larger. At the same gas pressure, the particle size increased with the evaporation temperature.

The processes leading to the formation of small metal clusters in the gas seem not to be very well understood. It is generally assumed that the atoms escaping from the evaporation source are cooled down by collisions with the atoms of the background gas. In a conventional vacuum of $10^{-5}$ Torr the mean free path of the metal vapour atoms is so large that they can reach a substrate more than $10 \mathrm{~cm}$ away without a significant number of collisions; at a pressure of 1 Torr of the gas the atoms are scattered by collisions with the atoms of the background gas after an average distance of the order of 
$50 \mu \mathrm{m}$. Due to the rather good thermal contact of the background gas with the colder surroundings, a thermal gradient is built up near the evaporation source, and unlike the case of a diverging beam in vacuum, collisions can now take place between the metal atoms themselves: When this gradient is steep enough, there will be a region where the metal density exceeds the saturated vapour density and nucleation of particles may occur. Subsequent growth takes place by addition of single atoms and by collisions of entire clusters; the smaller clusters with their higher mobility will have the biggest chance to be trapped. Granqvist and Buhrman [270,271] have studied the size distribution of metal particles obtained with this technique, and they found that the logarithm of the particle volume was distributed very accurately according to a Gaussian distribution. They concluded that the growth was dominated by coalescence of clusters. The size distributions published by other authors do indeed show a tail towards large particle sizes, characteristic for particle coalescence. This log-normal size distribution for spherical particles of diameter $d$ is given by

$$
f(d)=\frac{1}{\sqrt{2 \pi} \ln (\sigma)} \exp \left(-\frac{(\ln (d)-\ln (\bar{d}))^{2}}{2(\ln (\sigma))^{2}}\right) .
$$

The standard deviation was found to be $\sigma=1.48 \pm 0.12$ for all the samples prepared, irrespective of the kind of metal, the mean particle size, or the details of the method of evaporation. It seems that the half width of the size distribution is intrinsically rather large. The spread in particle size is larger than with some of the other available particle preparation techniques. On the other hand, the gas evaporation technique seems to be applicable for most materials. Apart from the twenty two metals mentioned in the review paper by Uyeda [272] on the work of several Japanese research groups, particles of several other metals, as $\mathrm{Na}, \mathrm{Li}$ and $\mathrm{Gd}$, were prepared as well for the study of size effects. Gen and coworkers have demonstrated that it is even possible to prepare particles of $\mathrm{Ag} / \mathrm{Cu}$ alloy [273]; similarly Tasaki et al. found that the composition of particles of several alloys of $\mathrm{Fe}$ with $\mathrm{Ni}, \mathrm{Cu}, \mathrm{Si}, \mathrm{Cr}, \mathrm{Gd}$ and $\mathrm{Ho}$ prepared with this gas evaporation technique was roughly the same as the one of the mother alloy [274].

Although small particles can be prepared of any metal with a suitably low melting point, only in a few investigations a mean particle size well below $10 \mathrm{~nm}$ was found; very small particles were obtained of aluminium [116-118, 275, 276], copper [133, 135], indium [174, 177], nickel [277], palladium [278] and vanadium [243].

It was noted that the size distribution was considerably broader when the particles were not produced at a constant evaporation temperature $[268,269]$. Therefore, in most experimental set-ups a shutter is used, and the evaporation source and quantity of metal is chosen in such a way that the change of temperature during evaporation is rather small; sometimes, even a temperature stabilized oven was used $[174,177,270,271,279]$.

In the simple set-up with a stationary inert gas used by Yatsuya geometrical effects have been observed. Therefore, great care must be taken to collect particles from the inner zone only where the size distribution is rather narrow and well defined. When the pressure of the background gas and the evaporation temperature are chosen in such a way that the inner zone extends beyond the walls of the evaporation chamber, proper collection will probably be guaranteed. As coalescence may occur also after the particles have been deposited onto the substrate or onto the walls of the evaporation chamber, these surfaces must be carefully shielded from the radiation heat of the evaporation source, and cooled with water or even with liquid nitrogen [116-118, 276, 277, 280].

Proper precautions, low pressure helium gas, and a low evaporation rate should give the best chance to produce well defined small particles when the gas evaporation method is used. 


\subsubsection{Preparation of samples}

For the study of QSE, samples are required that contain many more particles than needed for the electron microscope investigations, while the size distribution should remain as narrow as possible. Most authors simply collect the deposits of metallic particles from the wall of the evaporation chamber. In the case of aluminium, it is then possible to isolate the particles from each other by admitting oxygen during the production of the particles, or after deposition [116-118]; other workers added oil as a matrix material [277]. Some authors collected the particles on cooled substrates [270,271] and covered the deposits with paraffin to prevent oxidation of the metal clusters [145, 281]. A very elegant procedure was reported by Wada and Ichikawa [280]: The particles were deposited on the frozen surface of an organic solvent covering the walls of the evaporation chamber; after deposition of the particles the solvent was warmed to room temperature so that the particles were trapped well isolated from each other, and the solvent was then again frozen with liquid nitrogen. The concentration of particles in the solvent could be increased by using successive evaporation cycles.

Morozov, Naumenko and Petinov prepared samples with a high packing density $(\eta \simeq 0.6)$ by pressing small tin particles together into the form of tablets; the particles were isolated by oxidation, or by covering them chemically with an insulating layer [282]. Very often particles were mixed with paraffin, and volume concentrations up to 0.02 have been reported; the suspension of the particles in molten paraffin was then treated with ultrasonics and cooled rapidly in order to ensure a uniform distribution of the particles over the volume of the specimen $[283,284]$.

\subsubsection{Flowing inert gas systems}

Continuous operation and easier particle handling can be obtained with the use of a flowing inert gas system. In the simple set-up used by Suzdalev and coworkers [284], the particles were carried by the flowing inert gas and the stream was then drawn through a filter to collect the particles; subsequently, the particles were shaken into a glass ampoule and mixed with paraffin. Gen and Petinov [141] collected the particles directly by passing the aerosol through molten paraffin. This method was also used by Meier and Wyder [174] to prepare suspensions of indium particles in paraffin. The set-up used is shown in fig. 5.6: A stream of pure helium gas is drawn through a temperature stabilized oven where the metal

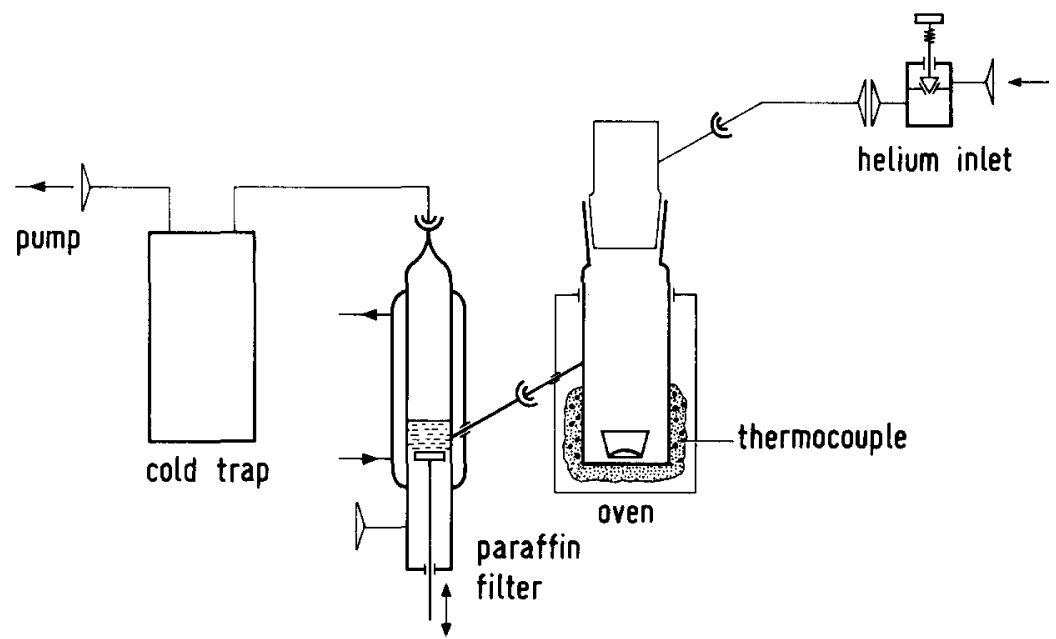

Fig. 5.6. Flowing inert gas system for the production of small metallic particles with the gas evaporation technique. The small metal particles are carried by the flowing helium gas and deposited in a filter of molten paraffin. (From Meier [250].) 


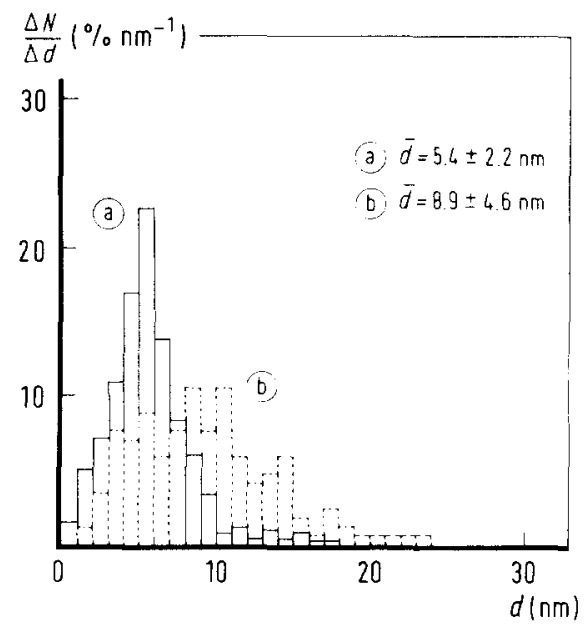

Fig. 5.7. Two typical size distributions of indium particles as prepared with the set-up shown in fig. 5.6.

is evaporated; the flow can be regulated with a needle valve at the inlet, the setting of this valve determines also the pressure of the helium gas in the oven. Part of the particles carried by the stream of helium are retained in the paraffin filter which is kept slightly above the solidification temperature of the paraffin by means of a temperature regulated water supply. A typical size distribution of particles prepared with this set-up is shown in fig. 5.7; in all these samples, a significant excess of very small particles was found when compared to the log-normal size distribution proposed by Granqvist and Buhrman [270, 271].

An oven of a more sophisticated design was used by Eversole and Broida [285]. A flow of heated inert gas was passed along the evaporation source, carrying the hot metal vapour through a nozzle to colder regions where nucleation and growth of the particles took place. Similarly, in the set-up used by Rappaz and Faes [279], the particles were produced in the inert background gas and then carried with the gas stream through a small diaphragm into a vacuum chamber where they could be deposited onto a cooled substrate, together with a suitable matrix material (e.g. carbondioxide).

The scheme reported by Yatsuya, Mihama and Uyeda [286] allows continuous operation as for the flowing inert gas systems, but in their system the metal is evaporated in vacuum onto a slowly rotating plate, covered with a thin layer of silicon oil. Small metallic particles grow at its surface (as with flash evaporation) and are subsequently trapped in the oil. Oil is fed at the center of the rotating disc, it flows outwards due to the centrifugal force, and is then collected in a container. The size of the particles obtained with this scheme depends both on the viscosity of the oil and the evaporation rate and seems to be considerably smaller $(d \ll 10 \mathrm{~nm})$ than obtained with the simple gas evaporation technique.

\subsubsection{Particles prepared in a beam geometry}

It is well known that clusters are formed on adiabatic expansion of an unsaturated vapour in an ultrasonic jet with a molecular beam set-up [287-290]. Large clusters of the inert gases are obtained easily, but in a metal beam it is very difficult to obtain a reasonable ratio of clusters to single atoms. Very high levels of supersaturation must be reached to produce nucleation into clusters of metal vapour. However, it is possible to produce metallic clusters with the seeded beam technique [291-294]; here inert gas is added to the metal vapour prior to jet expansion. In fact, this particle preparation technique is very similar to the more conventional gas-evaporation technique as discussed before in 
sections 5.3.1 and 5.3.3. Small clusters of potassium were produced successfully with this beam technique [292], and the production of clusters of various metals (such as indium, lead and bismuth) was extensively discussed by Stein and Yokozeki [293]. Very recently, using the seeded beam technique, Sattler and coworkers [294] were able to produce measurable quantities of metal clusters containing up to 500 atoms; in addition, they showed that the metallic particles are accessible for experimental investigations if they are separated according to their mass using a time of flight (TOF) arrangement. The use of a molecular beam set-up gives new and very promising possibilities, and it is to be expected that this technique will be used as the most elegant and clean way to produce well defined small particles for the study of QSE in the near future. Some basic experiments as electron diffraction $[290,293]$ and Stern-Gerlach deflection [292], have already been carried out very successfully. This field of research is rapidly growing, as can be seen e.g. from the proceedings of the International Meetings on Small Particles $[5,6]$.

\subsection{Particles prepared by nucleation and growth in a matrix}

\subsubsection{Photosensitive and stained glasses}

It was discovered long ago by Faraday that the beautiful colours of some types of glasses were due to finely dispersed clusters of gold. The optical properties of glasses containing small clusters of gold [21], or silver $[22,24,25,28,29]$ have repeatedly been investigated. Moreover, this sort of samples has been used to look for the existence of possible quantum size effects in the electric polarizability as predicted by Gor'kov and Eliashberg [4]. This was done by Dupree and Smithard [170] for silver particles in photosensitive glass, and by Meier and Wyder [169] for similar gold particles.

Meier and Wyder have used a recipe given by Maurer [295]: Small amounts of gold $(0.1 \%$ to $1.0 \%$ by weight, in the form of $\mathrm{HAuCl}_{4} \cdot n \mathrm{H}_{2} \mathrm{O}$ ) were added to the components of a glass consisting of $71.5 \%$ $\mathrm{SiO}_{2}, 23 \% \mathrm{Na}_{2} \mathrm{O}, 4 \% \mathrm{Al}_{2} \mathrm{O}_{3}, 1 \% \mathrm{ZnO}, 0.13 \% \mathrm{CeO}_{2}$, and $0.3 \% \mathrm{Sb}_{2} \mathrm{O}_{3}$. This mixture was heated to $1400^{\circ} \mathrm{C}$ for 8 hours, and after cooling down, a colourless and transparent glass was obtained. However, if the glass is first irradiated with ultraviolet light, it will become ruby-red when heated. The nucleation and growth of the gold particles were discussed in detail by Stookey [296] and Maurer [295]: When the glass is irradiated with ultraviolet light, the photosensitive agent $\mathrm{CeO}_{2}$ can reduce some of the gold ions; at ambient temperature, the gold atoms will remain fixed in the glass matrix, but when the glass is heated they can act as nuclei for the growth of metallic particles. In this way, the number of nucleation sites can be controlled with the dose of the radiation. As the glass is strongly absorbing in the ultraviolet, it is preferable to use gamma-rays to obtain a more homogeneous irradiation and consequently a homogeneous concentration of nucleation sites in the whole volume of the sample. When after the irradiation the sample is heated to the softening temperature of the glass (approximately $530^{\circ} \mathrm{C}$ ), gold and antimony ions can diffuse through the lattice and they will eventually be trapped by nuclei or clusters. The antimony, which acts as a thermoreducing agent, will then reduce the gold ions according to the redox reaction

$$
\mathrm{Sb}^{+3}+2 \mathrm{Au}^{+} \rightleftarrows \mathrm{Sb}^{+5}+2 \mathrm{Au}^{0}
$$

The growth process is very slow. It is limited by the diffusion of $\mathrm{Au}^{+}$and may be stopped at will by cooling the sample down to room temperature. Therefore, the number of particles can be controlled with the radiation dose, and the size of the particles will be determined mainly by the duration of the 
annealing process. The average diameter of the particles can be increased in several successive steps for one sample until the gold is depleted.

The concentration of gold particles is severely limited by the total amount of gold which can be added to the glass-melt: Already at a concentration of $1 \%$, Meier and Wyder observed a considerable broadening of the size distribution; this was attributed to the fact that still new nuclei were formed spontaneously during the annealing process. With a similar process, Smithard and Dupree [170] prepared samples with a volume fraction of silver up to $2.5 \times 10^{-4}$. They irradiated their samples with ultraviolet light in glasses carefully cut to slices of $0.5 \mathrm{~mm}$ thickness. This process of nucleation and growth of silver particles in photosensitive glasses was recently discussed again by Kreibig [297]. More stages of the growth process were now identified: In the first stage, the $\mathrm{Ag}^{\mathrm{o}}$ centers, formed after the irradiation, quickly coagulate to clusters containing of the order of hundred atoms; this process is completed after the first few minutes of tempering. After a second intermediate growth stage of which the growth kinetics are still unclear, a third stage was identified where the growth was determined by the process of diffusion limited growth mentioned above. Therefore, it has to be concluded that it is impossible to prepare samples with extremely small particles using this technique, despite the very slow growth of the particles.

Samples containing up to $1 \%$ by volume of silver particles were prepared by staining glass at $600^{\circ} \mathrm{C}$ in a bath of $\mathrm{AgCl}$ [170]. In this case, the silver concentration in the samples was strongly correlated with the $\mathrm{Sb}_{2} \mathrm{O}_{3}$ content of the glass; it was observed that $\mathrm{Ag}^{+}$diffuses into the glass to a concentration of approximately $0.5 \%$ by weight. If the glass contains antimony, the silver ions will be reduced and precipitate into silver clusters. The process comes to an end when the antimony is exhausted, and a coloured zone proceeded slowly through the glass sample, giving the same particle concentration and average particle size throughout the sample.

\subsubsection{Simultaneous evaporation of metal and matrix}

The method of simultaneous evaporation of the metal and a supporting matrix which was used by Borel and coworkers [146, 154-156] to prepare small metal particles shows some similarity with the technique discussed in section 5.4.1 above. The metal was evaporated onto a nitrogen cooled surface, and simultaneously, $\mathrm{CO}_{2}$ or benzene was condensed from the gas phase. When the samples were annealed at $195 \mathrm{~K}$, the metal atoms, dispersed in the matrix, precipitated and formed small particles with a diameter of the order of $1 \mathrm{~nm}$. The presence of these very small crystallites may explain the success in observing CESR in such samples, in accordance with the theoretical predictions, whereas no resonance signals were observed in silver particles dispersed in a glass matrix [29] or in colloidal gold particles prepared in the author's laboratory.

Very small clusters can be formed when silver atoms or other metals are dispersed in inert gas matrices $[298,299]$. When the silver is dispersed to a very low concentration in argon, very small clusters of $\mathrm{Ag}_{n}$ (with $n \leq 10$ ) are formed which could be identified by spectroscopic techniques. For a high concentration of silver in the argon matrix, colloidal particles with a diameter in the order of $1 \mathrm{~nm}$ were formed which showed the broad absorption band typical for small metallic particles [299].

\subsubsection{Alkali halide crystals}

Alkali halide crystals containing small metallic particles have been the object of several investigations. Charvolin et al. [112], Taupin [115], and Watts and Cousins [144] studied the properties of colloidal lithium clusters in lithium-fluoride by means of ESR and NMR techniques. Irradiation of lithium-fluoride with thermal neutrons results in lattice defects and in interstitial lithium atoms. These 
lithium atoms tend to coagulate when the radiation dose exceeds $3 \times 10^{21}$ neutrons $/ \mathrm{m}^{2}[113,300]$. At room temperature, and at this dose of irradiation plate like clusters of lithium are formed, several atomic layers thick and with an area of the order of $25 \mathrm{~nm}^{2}$. When the samples are annealed at $200^{\circ} \mathrm{C}$, the platelets disappear and crystallites are formed with a lower surface to volume ratio. When the radiation dose is higher (over $5 \times 10^{22}$ neutrons $/ \mathrm{m}^{2}$ ), globular clusters are formed with a diameter in the size range from 10 to $100 \mathrm{~nm}$; when the crystal is held at $77 \mathrm{~K}$, however, also in this case of high irradiation the formation of platelets is favoured.

It is well known that metal colloids are formed in additively coloured alkali halide crystals. These crystals are additively doped by heating to 600 or $700^{\circ} \mathrm{C}$ in alkali metal vapour and in complete darkness; then they are quenched to room temperature. When such crystals are then heated in the dark, and annealed at temperatures between 150 and $450^{\circ} \mathrm{C}$, the absorption peak due to the F-centers shifts to longer wavelengths: This behaviour is attributed to the formation of small clusters of the alkali metal. Colloidal metal particles develop when the initial F-center concentration exceeds a typical value of $10^{23} \mathrm{~m}^{-3}$.

The mechanism of the growth of these microcrystals was studied in great detail by Calleja and Agullo-Lopez [301]. In KCl, potassium colloids with a mean particle diameter of $20 \mathrm{~nm}$ were formed at an annealing temperature of $250^{\circ} \mathrm{C}$, and the diameter increased to $100 \mathrm{~nm}$ for higher annealing temperatures. After a first stage of rapid clustering, the average size of the colloidal particles continued to increase slowly, at the expense of the number of particles. In addition, at higher temperatures a considerable fraction of the alkali metal atoms was lost due to diffusion of the metal out of the crystal.

The optical properties of alkali metal particles of the type discussed here have been studied by different workers $[30,302]$. Experiments have also been reported on silver particles formed in additively coloured $\mathrm{KCl}: \mathrm{Ag}[32,153]$. The size distribution of the silver colloids was determined directly with an electron microscope. The average diameter was found to be 33 and $46 \mathrm{~nm}$ for samples that had been annealed at $700^{\circ} \mathrm{C}$ for 30 and 60 minutes, respectively. These particle sizes observed are in good agreement with the model of diffusion limited growth used by Calleja and Agullo-Lopez [303, 304]. The size distribution of the alkali metal clusters was not determined directly by electron microscopy, but average sizes, deduced from optical measurements, are found to be in the range from a few nm up to $100 \mathrm{~nm}$.

\subsection{Impregnated porous materials}

A very simple impregnation technique was used to prepare small nickel particles in silicagel. Lindquist and coworkers [305] prepared nickel particles with an average diameter of $6 \pm 2 \mathrm{~nm}$ by impregnation of "W.R. Grace grade 950" silicagel with a solution of $\mathrm{NiNO}_{3}$; the sample was dried in air, and reduced with hydrogen at $540^{\circ} \mathrm{C}$. With similar techniques, supported nickel and platinum catalysts with particle size down to $2 \mathrm{~nm}$ have been prepared.

An extensive series of experiments has been performed on porous glasses filled with metals: Novotny et al. have studied lattice dynamical effects for small particles in porous glass by means of heat capacity [241,242] and neutron scattering [306] experiments. They prepared $2.2 \mathrm{~nm}$ diameter indium particles and lead particles with an average diameter of $2.2,3.7$ and $6.0 \mathrm{~nm}$, with a method that was used very successfully before by Watson [223,307]. Porous glass samples were prepared by leaching a phase separated alkali-borosilicate glass in such a way that the boron-rich phase was removed and a porous body was left. Typical porous glasses contain $96 \% \mathrm{SiO}_{2}, 3 \% \mathrm{~B}_{2} \mathrm{O}_{3}$, small amounts of $\mathrm{Na}_{2} \mathrm{O}, \mathrm{Al}_{2} \mathrm{O}_{3}$, and other oxides. The resulting porous glass may be thought of as consisting of a combination of loosely 
packed $\mathrm{SiO}_{2}$ spheres of diameter of the order of $30 \mathrm{~nm}$; the average porosity is 15 to $25 \%$. When the glass is immersed in a bath of molten metal with a pressure of the order of $10^{9} \mathrm{~Pa}$ metal can be forced into the pores. Because of the high uniformity of the pore diameter and consequently the narrow size distribution of the particles contained in the glass, these systems are very attractive for experiments on size effects. Note that because of the fact that the thermal contraction of the glass is much smaller than the contraction of the metallic particles, the surface of the particles might possibly be considered as free when the sample is cooled to low temperatures. On the other hand, the broadening of the X-ray diffraction lines indicates a coherence length larger than the particle size; therefore it must be concluded that the particles are not entirely free from one another. Watson [223,307] prepared samples impregnated with indium, lead and thallium. Bogomolov et al. [308,309] also prepared samples containing mercury, tin, and gallium.

A large number of inorganic crystals contain regular cavities or vacancies forming networks or parallel channels; e.g. for zeolites, the size of the cavities is in the range from 0.2 to $1.2 \mathrm{~nm}$. Usually, the sizes of the cavities are very uniform, although in general there can be different types of cavities with different size. Moreover, there are several types of zeolites available (such as $\mathrm{NaA}$-, or $\mathrm{NaX}$-zeolite), with a different three dimensional arrangement of the cavities. Several metals, as mercury and gallium, were forced into these cavities $[310,311]$ and the properties of the resulting particles were studied. In zeolites, the cavities are so small that particles can be obtained in the size range $d<1 \mathrm{~nm}$; in some of these cavities, clusters containing only a few atoms can be accommodated.

\section{Appendix I. Thermodynamic calculations for Kubo's small particle}

\section{AI.1. Set up of the partition function [312]}

If $E_{j}$ are the energy eigenvalues of the total electron system, the partition function is defined as

$$
Z=\sum_{j=1,2} \exp \left(-E_{j} / k T\right)
$$

In the case of noninteracting electrons, each $E_{j}$ can be represented as a sum of single particle energies $\varepsilon_{i}$

$$
E_{j}=\sum_{i} n_{i j} \varepsilon_{i}
$$

As there are $N$ electrons in the particle, exactly $N$ single electron states are occupied in each configuration:

$$
N=\sum_{i} n_{i j}, \quad \text { for all } j
$$

Consequently, the partition function $Z$ can be written (with $\beta=1 / k T$ ) as:

$$
N=\sum_{j}^{(N)} \exp \left(-\beta\left(n_{1 j} \varepsilon_{1}+n_{2 j} \varepsilon_{2}+\cdots\right)\right)
$$


where the meaning of $(N)$ in the summation symbol is, that each term in the sum must have exactly $N$ values $n_{i j}$ different from zero. As we are dealing with electrons, $n_{i j}=0$ or 1 , depending on whether state $\varepsilon_{i}$ is occupied or not. $Z$ is a function of the particle number $N$ and the temperature $T$ and will be called $Z(T, N)$. One can set up a generating function

$$
f(\lambda)=\sum_{N=0}^{\infty} Z(T, N) \lambda^{N}
$$

With the definition of $Z(T, N)$ one gets:

$$
f(\lambda)=\sum_{N=0}^{\infty}\left(\sum_{j}^{(N)} \exp \left(-\beta\left(n_{1 j} \varepsilon_{1}+n_{2 j} \varepsilon_{2}+\cdots\right)\right) \lambda^{\left(n_{1 j}+n_{2 j}+\cdots\right)}\right) .
$$

In $f(\lambda)$ all possible configurations $\exp \left(-\beta\left(n_{1 j} \varepsilon_{1}+n_{2 j} \varepsilon_{2}+\cdots\right)\right) \lambda^{\left(n_{1 j}+n_{2 j}+\cdots\right)}$ appear exactly once, but now without restriction for the total number of electrons. Therefore:

$$
f(\lambda)=\prod_{i}\left(\sum_{j} \exp \left(-\beta n_{i j} \varepsilon_{i}\right) \lambda^{n_{i j}}\right)=\prod_{i}\left(1+\lambda \exp \left(\beta \varepsilon_{i}\right)\right)
$$

Because $f(\lambda)$ is analytic in the whole complex plane, the residue theorem allows to project out $Z(T, N)$ from the physically meaningless $f(\lambda)$ :

$$
Z(T, N)=\frac{1}{2 \pi \mathrm{i}} \int_{\text {origin }} \frac{f(\lambda)}{\lambda^{N+1}} \mathrm{~d} \lambda
$$

In the presence of a magnetic field $H$, the single electron levels are redefined as:

$$
\begin{array}{ll}
\varepsilon_{i} \pm \mu_{0} \mu_{\mathrm{B}} H & i>0, \text { i.e. } \varepsilon_{i}>0 \\
\varepsilon_{0}=0 & \text { Fermi energy } \\
\varepsilon_{l} \pm \mu_{0} \mu_{\mathrm{B}} H=-\varepsilon_{l}^{\prime} \pm \mu_{0} \mu_{\mathrm{B}} H & l<0 \text {, i.e. } \varepsilon_{l}<0, \varepsilon_{l}^{\prime}>0 .
\end{array}
$$

With $H=0$, each state $\varepsilon_{i}$ is spin (i.e. twice) degenerate. $f(\lambda)$ can be written as:

$$
\begin{aligned}
Z(T, N)= & \frac{1}{2 \pi \mathrm{i}} \int \frac{\mathrm{d} \lambda}{\lambda^{N+1}} \prod_{i \geq 1}\left(1+\lambda \exp \left(-\beta\left(\varepsilon_{i}-\mu_{0} \mu_{\mathrm{B}} H\right)\right)\right) \prod_{j \geq 1}\left(1+\lambda \exp \left(-\beta\left(\varepsilon_{j}+\mu_{0} \mu_{\mathrm{B}} H\right)\right)\right) \\
& \times \prod_{k \leq 0}\left(1+\lambda \exp \left(-\beta\left(\varepsilon_{k}-\mu_{0} \mu_{\mathrm{B}} H\right)\right)\right) \prod_{l \leq 0}\left(1+\lambda \exp \left(-\beta\left(\varepsilon_{l}+\mu_{0} \mu_{\mathrm{B}} H\right)\right)\right) .
\end{aligned}
$$

The $(N+1) \lambda$ 's in the denominator are distributed on the terms with $k, l \leq 0$. When $N$ is an odd number, $(N+1)$ is even and there is for each factor one $\lambda$. When $N$ is even, there is one factor $\lambda$ left. Here appears in mathematical language the difference between particles with an even and an odd number of electrons. With $\lambda^{\alpha}$, which can take the values $\lambda$ or 1 , one can write: 


$$
\begin{aligned}
Z(T, N)= & \frac{1}{2 \pi \mathrm{i}} \int \frac{\mathrm{d} \lambda}{\lambda^{\alpha}} \prod_{i \geq 1}\left(1+\lambda \exp \left(-\beta\left(\varepsilon_{i}-\mu_{0} \mu_{\mathrm{B}} H\right)\right)\right) \prod_{j \geq 1}\left(1+\lambda \exp \left(-\beta\left(\varepsilon_{j}+\mu_{0} \mu_{\mathrm{B}} H\right)\right)\right) \\
& \times \prod_{k \leq 0}\left(\frac{1}{\lambda}+\exp \left(-\beta\left(\varepsilon_{k}-\mu_{0} \mu_{\mathrm{B}} H\right)\right)\right) \prod_{i \leq 0}\left(\frac{1}{\lambda}+\exp \left(-\beta\left(\varepsilon_{l}+\mu_{0} \mu_{\mathrm{B}} H\right)\right)\right)
\end{aligned}
$$

Putting $\gamma=\exp \left(\beta \mu_{0} \mu_{\mathrm{B}} H\right)$ and making use of eq. (AI.9) one gets after slight rearrangement:

$$
\begin{aligned}
Z(T, N)= & \frac{\mathrm{e}^{\beta E(0)}}{2 \pi \mathrm{i}} \int \frac{\mathrm{d} \lambda}{\lambda^{\alpha}}\left(1+\frac{\gamma}{\lambda}\right)\left(1+\frac{1}{\lambda \gamma}\right) \prod_{i \geq 1}\left(1+\lambda \gamma \exp \left(-\beta \varepsilon_{i}\right)\right) \\
& \times \prod_{j \geq 1}\left(1+\frac{\lambda}{\gamma} \exp \left(-\beta \varepsilon_{j}\right)\right) \prod_{k \leq-1}\left(\frac{1}{\lambda \gamma} \exp \left(-\beta \varepsilon_{k}^{\prime}\right)+1\right) \prod_{l \leq-1}\left(\frac{\gamma}{\lambda} \exp \left(-\beta \varepsilon_{l}^{\prime}\right)+1\right)
\end{aligned}
$$

$E(0)$ is the total energy with all states occupied up to the Fermi energy $\varepsilon_{\mathrm{F}}$ : for the "even" case: $E(0)=\Sigma_{l \leq 0} 2 \varepsilon_{l}^{\prime}$. $\alpha$ can take two values:

$$
\begin{array}{ll}
\alpha=0, & \text { for "odd" case } \\
\alpha=1, & \text { for "even" case . }
\end{array}
$$

The free energy is given by:

$$
F(T, N)=-k T\langle\ln Z(T, N)\rangle
$$

where the brackets indicate an average over the statistical level distribution of eq. (3.18). An additional averaging must be carried out when an assembly of particles with unequal size is considered.

\section{AI.2. Some averages}

We present expressions for some average values which will be needed later. For an arbitrary function $\phi(\varepsilon)$, the following theorems on average values $\langle\ldots\rangle$ are valid if the nearest level spacings are Poisson distributed:

$$
\left\langle\sum_{i>0} \phi\left(\varepsilon_{i}\right)\right\rangle=\left\langle\sum_{k<0} \phi\left(\varepsilon_{k}^{\prime}\right)\right\rangle=\int_{0}^{\infty} \frac{1}{\delta} \phi(\varepsilon) \mathrm{d} \varepsilon
$$

This can easily be seen by considering the average

$$
\left\langle\mathrm{e}^{-s \boldsymbol{E}_{i}}\right\rangle=\left\langle\exp \left(-s \sum_{j=1}^{i} \Delta_{j}\right)\right\rangle=\left\langle\mathrm{e}^{-s \Delta_{1}} \mathrm{e}^{-s \Delta_{2}} \ldots \mathrm{e}^{-s \Delta_{i}}\right\rangle .
$$

Because the $\Delta_{j}$ are independent of each other, one has

$$
\left\langle\mathrm{e}^{-s \varepsilon_{i}}\right\rangle=\left\langle\mathrm{e}^{-s \Delta_{1}}\right\rangle\left\langle\mathrm{e}^{-s \Delta_{2}}\right\rangle \ldots\left\langle\mathrm{e}^{-s \Delta_{i}}\right\rangle .
$$


Each factor on the right is equal to

$$
\left\{\frac{1}{\delta} \int_{0}^{\infty} \mathrm{e}^{-s \Delta} \mathrm{e}^{-\Delta / \delta} \mathrm{d} \Delta\right\}=(1+s \delta)^{-1}
$$

Consequently, if $\operatorname{Re} s>0:\left\langle\exp \left(-s \varepsilon_{i}\right)\right\rangle=(1+s \delta)^{-i} ;$ furthermore, if $1 /(1+s \delta)<1$,

$$
\left\langle\sum_{i=1}^{\infty} \exp \left(-s \varepsilon_{i}\right)\right\rangle=\sum_{i=1}^{\infty}(1+s \delta)^{-i}=\frac{1}{s \delta}
$$

Assuming that there is an integral representation of $\phi(\varepsilon)$ of the form

$$
\phi(\varepsilon)=\int_{a}^{b} \mathrm{e}^{-s \varepsilon} \psi(s) \mathrm{d} s
$$

then:

$$
\begin{aligned}
\left\langle\sum_{i=1}^{\infty} \phi\left(\varepsilon_{i}\right)\right\rangle & =\left\langle\sum_{i=1}^{\infty} \int_{a}^{b} \mathrm{e}^{-s \varepsilon_{i}} \psi(s) \mathrm{d} s\right\rangle=\int_{a}^{b} \frac{1}{s \delta} \psi(s) \mathrm{d} s \\
& =\int_{0}^{\infty} \frac{1}{\delta}\left(\int_{a}^{b} \mathrm{e}^{-s \varepsilon} \psi(s) \mathrm{d} s\right) \mathrm{d} \varepsilon=\int_{0}^{\infty} \frac{1}{\delta} \phi(\varepsilon) \mathrm{d} \varepsilon
\end{aligned}
$$

and this proves the theorem.

Similarly for $\phi\left(\varepsilon_{1}, \varepsilon_{2}\right)$ :

$$
\begin{aligned}
& \left\langle\sum_{i=1}^{\infty} \sum_{j>i} \phi\left(\varepsilon_{i}, \varepsilon_{j}\right)\right\rangle=\sum_{k=-1}^{-\infty} \sum_{l<k} \phi\left(\varepsilon_{k}^{\prime}, \varepsilon_{l}^{\prime}\right)=\int_{0}^{\infty} \frac{\mathrm{d} \varepsilon}{\delta} \int_{\varepsilon}^{\infty} \frac{\mathrm{d} \varepsilon^{\prime}}{\delta} \phi\left(\varepsilon, \varepsilon^{\prime}\right) \\
& \left\langle\sum_{i>0} \sum_{k<0} \phi\left(\varepsilon_{i}, \varepsilon_{k}^{\prime}\right)\right\rangle=\int_{0}^{\infty} \frac{\mathrm{d} \varepsilon}{\delta} \int_{0}^{\infty} \frac{\mathrm{d} \varepsilon^{\prime}}{\delta} \phi\left(\varepsilon, \varepsilon^{\prime}\right) .
\end{aligned}
$$

\section{AI.3. High temperature limit $\beta \delta \ll 1$ (no QSE)}

The product terms of eq. (AI.12) can be written as $\mathrm{e}^{\Phi(\lambda, y, \boldsymbol{\beta})}$, where

$$
\begin{aligned}
\Phi(\lambda, \gamma, \beta)= & \sum_{i=1}^{4} \Phi_{i}=\sum_{i \geq 1}\left\{\ln \left(1+\lambda \gamma \mathrm{e}^{-\beta \varepsilon_{i}}\right)+\ln \left(1+\frac{\lambda}{\gamma} \mathrm{e}^{-\beta \varepsilon_{i}}\right)\right\} \\
& +\sum_{k \leq-1}\left\{\ln \left(1+\frac{1}{\lambda \gamma} \mathrm{e}^{-\beta \varepsilon_{k}^{i}}\right)+\ln \left(1+\frac{\gamma}{\lambda} \mathrm{e}^{-\beta \varepsilon_{i}}\right)\right\}
\end{aligned}
$$


Therefore:

$$
Z(T, N)=\frac{\mathrm{e}^{\beta E(0)}}{2 \pi \mathrm{i}} \int \frac{\mathrm{d} \lambda}{\lambda^{\alpha}}\left(1+\frac{\gamma}{\lambda}\right)\left(1+\frac{1}{\lambda \gamma}\right) \mathrm{e}^{\Phi(\lambda, \gamma, \beta)} .
$$

When $\beta \delta \ll 1$, the sums in eq. (AI.21) may be replaced by integrals; the function $\mathrm{e}^{-\boldsymbol{\beta} \varepsilon}$ stays practically constant between $\exp \left(-\beta \varepsilon_{i}\right)$ and $\exp \left(-\beta \varepsilon_{i+1}\right)$, where $\varepsilon_{i+1}-\varepsilon_{i}$ is (on the average) equal to $\delta$. For instance, the first term in $\Phi(\lambda, \gamma, \beta)$ is

$$
\Phi_{1}(\lambda, \gamma, \beta)=\sum_{i \geq 1} \ln \left(1+\lambda \gamma \mathrm{e}^{-\beta \varepsilon_{i}}\right) \simeq \int \frac{1}{2} \ln \left(1+\lambda \gamma \mathrm{e}^{-\beta \varepsilon}\right) \rho\left(\varepsilon_{\mathrm{F}}\right) \mathrm{d} \varepsilon
$$

where $\rho\left(\varepsilon_{\mathrm{F}}\right)$ is the density of states at the Fermi energy $\varepsilon_{\mathrm{F}}\left(\rho\left(\varepsilon_{\mathrm{F}}\right)=2 / \delta\right)$. Over the range where the integral is substantially different from zero, $\rho(\varepsilon)$ has been assumed to be constant and equal to $\rho\left(\varepsilon_{\mathrm{F}}\right)$. $\Phi(\lambda, \gamma, \beta)$ is an analytic function in the complex $\lambda$-plane and has a saddle point at $\lambda=1$ because $(\partial \Phi / \partial \lambda)_{\lambda=1}=0$. (For an exposition of this saddle point method, see e.g. [313].) The value of $\Phi(1, \gamma, \beta)$ can be made arbitrarily large for $\beta \delta \rightarrow 0$. By putting $x=\mathrm{e}^{-\beta \varepsilon}$ one gets, again for $\Phi_{1}$

$$
\Phi_{1}(\lambda, \gamma, \beta)=\frac{1}{\delta} \int_{0}^{\infty} \ln \left(1+\lambda \gamma \mathrm{e}^{-\beta \varepsilon}\right) \mathrm{d} \varepsilon=\frac{1}{\beta \delta} \int_{0}^{1} \frac{1}{x} \ln (1+\lambda \gamma x) \mathrm{d} x=\frac{1}{\beta \delta} \tilde{\Phi}_{1}(\lambda \gamma)
$$

The remaining terms $\Phi_{i}$ show the same behaviour. Therefore, when the contour integral is replaced by the value of the integral at $\lambda=1$ and the logarithm is formed in order to get the free energy, in the high temperature limit $\beta \delta \ll 1$ only the factor $\mathrm{e}^{\boldsymbol{\Phi ( \lambda , \gamma , \beta )}}$ in eq. (AI.22) contributes significantly. Consequently:

$$
\ln Z(T, N) \simeq \Phi(1, \gamma, \beta)
$$

Using eqs. (AI.14) and (AI.21) one obtains for $\lambda=1$ :

$$
\langle\ln Z(T, N)\rangle=\frac{2}{\delta} \int_{0}^{\infty} \mathrm{d} \varepsilon\left\{\ln \left(1+\gamma \mathrm{e}^{-\beta \varepsilon}\right)+\ln \left(1+\frac{1}{\gamma} \mathrm{e}^{-\beta \varepsilon}\right)\right\} .
$$

For $\gamma=1$ (i.e. $H=0$ ) one gets

$$
\langle\ln Z(T, N)\rangle=\frac{4}{\delta} \int_{0}^{\infty} \mathrm{d} \varepsilon \ln \left(1+\mathrm{e}^{-\beta \varepsilon}\right)=\frac{\pi^{2}}{3 \beta \delta}
$$

and the heat capacity becomes

$$
C_{V}=-T \partial^{2} F / \partial T^{2}=\frac{1}{3} \pi^{2} k^{2} T \rho\left(\varepsilon_{\mathrm{F}}\right)
$$

which is the well known bulk result [80]. Similarly for $\beta \delta \rightarrow 0$ and $H \neq 0$ 


$$
\begin{aligned}
Z(H, T, N)= & \frac{\mathrm{e}^{\beta E(0)}}{-2 \pi \mathrm{i}} \int \frac{\mathrm{d} \lambda}{\lambda^{\alpha}}\left(1+\frac{\gamma}{\lambda}\right)\left(1+\frac{1}{\lambda \gamma}\right) \exp \left(\frac { 1 } { \delta } \int _ { 0 } ^ { \infty } \mathrm { d } \varepsilon \left\{\ln \left(1+\lambda \gamma \mathrm{e}^{-\beta \varepsilon}\right)\right.\right. \\
& \left.\left.+\ln \left(1+\frac{\lambda}{\gamma} \mathrm{e}^{-\beta \varepsilon}\right)+\ln \left(1+\frac{\gamma}{\lambda} \mathrm{e}^{-\beta \varepsilon}\right)+\ln \left(1+\frac{1}{\lambda \gamma} \mathrm{e}^{-\beta \varepsilon}\right)\right\}\right)
\end{aligned}
$$

With the method of steepest descent [313], we only need to consider the contribution for $\lambda=1$ in the integrand of $\mathrm{e}^{\Phi(\boldsymbol{\lambda}, \boldsymbol{\gamma}, \boldsymbol{\beta})}$ so that we can write:

$$
\langle\ln Z(H, T, N)\rangle=\beta E(0)+\ln \left(\gamma+\frac{1}{\gamma}\right)+\frac{2}{\delta} \int_{0}^{\infty} \mathrm{d} \varepsilon\left\{\ln \left(1+\gamma \mathrm{e}^{-\beta \varepsilon}\right)+\ln \left(1+\frac{1}{\gamma} \mathrm{e}^{-\beta \varepsilon}\right)\right\} .
$$

The second term comes in only for $\alpha=0$ (odd number of electrons), so that:

$$
\begin{aligned}
\chi & =-\frac{1}{\mu_{0}} \frac{\partial^{2} F}{\partial H^{2}}=\frac{k T}{\mu_{0}} \frac{\partial^{2}}{\partial H^{2}}\langle\ln Z(H, T, N)\rangle \\
& =\frac{\partial}{\partial H}\left\{\mu_{\mathrm{B}}\left(\frac{\gamma-1 / \gamma}{\gamma+1 / \gamma}\right)+\frac{2}{\delta} \mu_{\mathrm{B}} k T \ln \left(\frac{1+\gamma}{1+1 / \gamma}\right)\right\} \quad(\text { for } \gamma \rightarrow 1) \\
& =\frac{\mu_{0} \mu_{\mathrm{B}}^{2}}{k T}+\frac{2}{\delta} \mu_{0} \mu_{\mathrm{B}}^{2}
\end{aligned}
$$

The first term in eq. (AI.31) is negligible compared with the second when $\beta \delta \ll 1$, and the usual result for the bulk Pauli spin paramagnetism is obtained [314]

$$
\chi=\mu_{0} \mu_{\mathrm{B}}^{2} \rho\left(\varepsilon_{\mathrm{F}}\right) .
$$

\section{AI.4. Low temperature limit $\beta \delta \gg 1$ (QSE)}

The partition function of eq. (AI.12) will be averaged over the Poisson level distribution of eq. (3.18); it is then possible to expand $\langle Z(T, N)\rangle$ in a power series of the argument $(\beta \delta)^{-1}$; as we are interested in the situation with $\beta \delta \gg 1$, we calculate only the first term and neglect terms of the order $(\beta \delta)^{-2}$ and of higher order. The corrections due to the $(\beta \delta)^{-2}$-term are explicitly calculated in Kubo's paper [3]. From eq. (AI.14), the following averages are obtained:

$$
\left\langle\sum_{i>0} \exp \left(-\beta \varepsilon_{i}\right)\right\rangle=\left\langle\sum_{l<0} \exp \left(-\beta \varepsilon_{l}^{\prime}\right\rangle=\int_{0}^{\infty} \exp (-\beta \varepsilon) \frac{\mathrm{d} \varepsilon}{\delta}=\frac{1}{\beta \delta} .\right.
$$

Similarly,

$$
\left\langle\sum_{i \geq 1} \sum_{j>i} \exp \left(-\beta\left(\varepsilon_{i}+\varepsilon_{j}\right)\right)\right\rangle=\left\langle\sum_{k \leq-1} \sum_{l<k} \exp \left(-\beta\left(\varepsilon_{k}^{\prime}+\varepsilon_{l}^{\prime}\right)\right)\right\rangle=\frac{1}{2(\beta \delta)^{2}}
$$


Going back to the original expression for the partition function $Z(T, N)$ (eq. (AI.12)) only those terms are collected which give averages of the order $(\beta \delta)^{-1}$ :

$$
\begin{aligned}
Z(T, N)= & \frac{\mathrm{e}^{\beta E(0)}}{2 \pi \mathrm{i}} \int \frac{\mathrm{d} \lambda}{\lambda^{\alpha}}\left(1+\frac{\gamma}{\lambda}\right)\left(1+\frac{1}{\lambda \gamma}\right)\left\{1+\left(\gamma+\frac{1}{\gamma}\right)\left(\lambda \sum_{i \geq 1} \exp \left(-\beta \varepsilon_{i}\right)\right.\right. \\
& \left.\left.+\frac{1}{\lambda} \sum_{k \leq-1} \exp \left(-\beta \varepsilon_{k}^{\prime}\right)\right)+\lambda^{2} \sum_{j \geq 1} \exp \left(-2 \beta \varepsilon_{j}\right)+\frac{1}{\lambda^{2}} \sum_{l \leq-1} \exp \left(-2 \beta \varepsilon_{l}^{\prime}\right)+\cdots\right\} .
\end{aligned}
$$

All additional terms contain more than one level $\varepsilon_{i}$ or $\varepsilon_{i}^{\prime}$. Using the fact that only the term with $1 / \lambda$ gives a contribution when the integration around the origin is carried out, one gets for $\alpha=0$ (odd number of electrons):

$$
\begin{aligned}
\left.Z(T, N)\right|_{\text {odd }} & =\frac{\mathrm{e}^{\beta E(0)}}{2 \pi \mathrm{i}} \int \frac{\mathrm{d} \lambda}{\lambda}\left\{\left(\gamma+\frac{1}{\gamma}\right)+\left(\gamma+\frac{1}{\gamma}\right)\left(\sum_{i \geq 1} \exp \left(-\beta \varepsilon_{i}\right)+\sum_{k \leq-1} \exp \left(-\beta \varepsilon_{k}^{\prime}\right)+\cdots\right)\right\} \\
& =\mathrm{e}^{\beta E(0)}\left\{\left(\gamma+\frac{1}{\gamma}\right)\left(1+\sum_{i \geq 1} \exp \left(-\beta \varepsilon_{i}\right)+\sum_{k \leq-1} \exp \left(-\beta \varepsilon_{k}^{\prime}\right)+\cdots\right)\right\}
\end{aligned}
$$

For $\alpha=1$ (even number of electrons) the analogous expression is

$$
\left.Z(T, N)\right|_{\text {even }}=\mathrm{e}^{\beta E(0)}\left\{1+\left(\gamma+\frac{1}{\gamma}\right)^{2} \sum_{j \geq 1} \exp \left(-\beta \varepsilon_{j}\right)+\sum_{l \geq 1} \exp \left(-2 \beta \varepsilon_{l}\right)+\cdots\right\}
$$

In making these expansions, it was assumed that $\gamma \ll \beta \delta$, i.e. $\mu_{0} \mu_{\mathrm{B}} H<\delta$; for $\gamma / \beta \delta>1$, terms of order higher than $(\beta \delta)^{-1}$ can no longer be neglected. The averaging procedure is now very simple:

$$
\begin{aligned}
\left\langle\left.\ln Z(T, N)\right|_{\text {odd }}\right\rangle & =\beta E(0)+\ln \left(\gamma+\frac{1}{\gamma}\right)+\left\langle\ln \left(1+\sum_{i \geq 1} \exp \left(-\beta \varepsilon_{i}\right)+\sum_{k \leq-1} \exp \left(-\beta \varepsilon_{k}^{\prime}\right)+\cdots\right)\right\rangle \\
& =\beta E(0)+\ln \left(\gamma+\frac{1}{\gamma}\right)+\left\langle\ln \left(1+\sum_{i \geq 1} \exp \left(-\beta \varepsilon_{i}\right)\right)\left(1+\sum_{k \leq-1} \exp \left(-\beta \varepsilon_{k}^{\prime}\right)\right)\right\rangle \\
& =\beta E(0)+\ln \left(\gamma+\frac{1}{\gamma}\right)+\frac{2}{\beta \delta} \int_{0}^{\infty} \mathrm{d} x \ln \left(1+\mathrm{e}^{-x}\right) \\
& =\beta E(0)+\ln \left(\gamma+\frac{1}{\gamma}\right)+\frac{\pi^{2}}{6 \beta \delta} .
\end{aligned}
$$

Here, use is made of the fact, that the expression $\left\langle\ln \left(\Sigma_{i \geq 1} \exp \left(-\beta \varepsilon_{i}\right) \Sigma_{k \leq-1} \exp \left(-\beta \varepsilon_{k}^{\prime}\right)\right)\right\rangle$ may be set equal to zero as it contains more than one series of levels. For $\alpha=1$ one obtains:

$$
\left\langle\left.\ln Z(T, N)\right|_{\text {even }}\right\rangle=\beta E(0)+\frac{1}{\beta \delta} \int_{0}^{\infty} \mathrm{d} x \ln \left(1+\left(\gamma+\frac{1}{\gamma}\right)^{2} \mathrm{e}^{-x}+\mathrm{e}^{-2 x}\right)
$$

Now we are able to give explicit expressions for the specific heat and the magnetization in the QSE 
limit. Taking $H=0$, one gets for the specific heat in the "odd" and "even" case respectively:

$$
\begin{aligned}
& C_{\text {odd }}=-T \frac{\delta^{2} F_{\text {odd }}}{\partial T^{2}}=-T \frac{\partial^{2}}{\partial T^{2}}\left(-\frac{1}{\beta} \frac{\pi^{2}}{6 \beta \delta}\right)=\frac{\pi^{2}}{3} \frac{k^{2}}{\delta} T=1.645 k^{2} T \rho\left(\varepsilon_{\mathrm{F}}\right) \\
& C_{\text {even }}=2.512 k^{2} T \rho\left(\varepsilon_{\mathrm{F}}\right) .
\end{aligned}
$$

These results have to be compared with the electronic specific heat of the bulk metal as given by eq. (AI.28).

To calculate explicitly the magnetization, the condition $\mu_{0} \mu_{\mathrm{B}} H \ll \delta$ has to be fulfilled. For the "odd" case one gets according to (AI.38):

$$
\begin{aligned}
M_{\mathrm{odd}} & =-\frac{1}{\mu_{0}} \frac{\partial F}{\partial H}=\frac{1}{\mu_{0}} k T \frac{\partial}{\partial H} \ln \left(\exp \left(\beta \mu_{0} \mu_{\mathrm{B}} H\right)+\exp \left(-\beta \mu_{0} \mu_{\mathrm{B}} H\right)\right) \\
& =\mu_{\mathrm{B}} \tanh \left(\mu_{0} \mu_{\mathrm{B}} H / k T\right)
\end{aligned}
$$

For $H \rightarrow 0$, the susceptibility is given by

$$
\chi_{\text {odd }}=\left.\frac{\partial M}{\partial H}\right|_{H=0}=\frac{\mu_{0} \mu_{\mathrm{B}}^{2}}{k T} .
$$

In the "even" case, the analogous quantities are given by:

$$
\begin{aligned}
& M_{\text {even }}=\frac{4 \mu_{\mathrm{B}}}{\beta \delta} \sinh \left(2 \beta \mu_{0} \mu_{\mathrm{B}} H\right) \int_{0}^{1} \frac{\underline{\mathrm{d} t}}{1+4 t \cosh ^{2}\left(\beta \mu_{0} \mu_{\mathrm{B}} H\right)+t^{2}} \\
& \chi_{\text {even }}=1.521 \mu_{0} \mu_{\mathrm{B}}^{2} \rho\left(\varepsilon_{\mathrm{F}}\right) .
\end{aligned}
$$

\section{Appendix II. Thermodynamic calculations for the particle with equal level spacing}

The canonical partition function of a particle with a constant and equal level spacing can be calculated exactly [88].

With the definition of Appendix I, the starting point to calculate the partition function is given by eq. (AI.12). Replacing $\lambda$ by $\mathrm{e}^{\mathrm{i} \phi}$ and $\gamma$ by $\mathrm{e}^{h}$, where $h=\beta \mu_{0} \mu_{\mathrm{B}} H$, one gets for the "odd" case $(\alpha=0)$ :

$$
\begin{aligned}
\mathrm{e}^{-\beta E(0)} Z(T, N)= & \frac{1}{2 \pi \mathrm{i}} \int_{0}^{2 \pi} \mathrm{ie}^{-\mathrm{i} \phi} \mathrm{d} \phi\left(1+\mathrm{e}^{-\mathrm{i} \phi+h}\right)\left(1+\mathrm{e}^{-\mathrm{i} \phi-h}\right) \\
& \times \prod_{n \geq 1}\left(1+\mathrm{e}^{\mathrm{i} \phi+h} \mathrm{e}^{-\beta \delta n}\right)\left(1+\mathrm{e}^{\mathrm{i} \phi-h} \mathrm{e}^{-\beta \delta n}\right)\left(1-\mathrm{e}^{-\beta \delta n}\right) \\
& \times \prod_{n \geq 1}\left(1+\mathrm{e}^{-\mathrm{i} \phi-h} \mathrm{e}^{-\beta \delta n}\right)\left(1+\mathrm{e}^{-\mathrm{i} \phi+h} \mathrm{e}^{-\beta \delta n}\right)\left(1-\mathrm{e}^{-\beta \delta n}\right) Z_{\mathrm{B}}^{2} .
\end{aligned}
$$


The factor $Z_{\mathrm{B}}^{2}=\prod_{n=1}^{\infty}\left(1-\mathrm{e}^{-\beta \delta n}\right)^{-2}$ compensates for the two factors $\prod_{n=1}^{\infty}\left(1-\mathrm{e}^{-\beta \delta n}\right)$ in eq. (AII.1). These factors are introduced in order to apply some properties of the $\theta$-function [315]:

$$
\frac{1}{q^{1 / 4} \cos Z} \sum_{n=0}^{\infty} q^{\left\{(n+1 / 2)^{2}\right\}} \cos ((2 n+1) Z)=\prod_{n=1}^{\infty}\left(1-q^{2 n}\right)\left(1+q^{2 n} \mathrm{e}^{+\mathrm{i} 2 Z}\right)\left(1+q^{2 n} \mathrm{e}^{-\mathrm{i} 2 Z}\right) .
$$

Putting $q^{2}=\mathrm{e}^{-\beta \delta}$ and $Z=\phi / 2+h / 2 \mathrm{i}$, one obtains from eq. (AII.2):

$$
\begin{aligned}
& \prod_{n=1}^{\infty}\left(1-\mathrm{e}^{-\beta \delta n}\right)\left(1+\mathrm{e}^{\mathrm{i} \phi+h} \mathrm{e}^{-\beta \delta n}\right)\left(1+\mathrm{e}^{-\mathrm{i} \phi-h} \mathrm{e}^{-\beta \delta n}\right) \\
& \quad=\frac{1}{\cos (\phi / 2+h / 2 \mathrm{i})} \sum_{n=0}^{\infty} \mathrm{e}^{-\beta \delta(n+1) n / 2} \cos \left((2 n+1)\left(\frac{\phi}{2}+\frac{h}{2 \mathrm{i}}\right)\right)
\end{aligned}
$$

Similarly, with $q^{2}=\mathrm{e}^{-\beta \delta}$ and $Z=\phi / 2-h / 2 \mathrm{i}$, one gets:

$$
\begin{aligned}
\prod_{n=1}^{\infty}\left(1-\mathrm{e}^{-\beta \delta n}\right)\left(1+\mathrm{e}^{\mathrm{i} \phi-h} \mathrm{e}^{-\beta \delta n}\right)\left(1+\mathrm{e}^{-\mathrm{i} \phi+h} \mathrm{e}^{-\beta \delta n}\right) \\
=\frac{1}{\cos (\phi / 2-h / 2 \mathrm{i})} \sum_{n=0}^{\infty} \mathrm{e}^{-\beta \delta(n+1) n / 2} \cos \left((2 n+1)\left(\frac{\phi}{2}-\frac{h}{2 \mathrm{i}}\right)\right) .
\end{aligned}
$$

The cos-terms in the denominator of eqs. (AII.3) and (AII.4) cancel (up to a factor $4 / \mathrm{e}^{\mathrm{i} \phi}$ ) with the factors $\left(1+\mathrm{e}^{-\mathrm{i} \phi+h}\right)\left(1+\mathrm{e}^{-\mathrm{i} \phi-h}\right)$ in eq. (AII.1). Eq. (AII.1) can now be written as:

$$
\begin{aligned}
\exp \left(-\beta E_{N}(0)\right) Z_{N}(T, H)= & \frac{1}{2 \pi} \int_{0}^{2 \pi} \mathrm{e}^{\mathrm{i} \phi} \mathrm{d} \phi \frac{4}{\mathrm{e}^{\mathrm{i} \phi}}\left\{\left(\sum_{n=0}^{\infty} \mathrm{e}^{-\beta \delta(n+1) n / 2} \cos \left((2 n+1)\left(\frac{\phi}{2}+\frac{h}{2 \mathrm{i}}\right)\right)\right)\right. \\
& \left.\times\left(\sum_{m=0}^{\infty} \mathrm{e}^{-\beta \delta(m+1) m / 2} \cos \left((2 m+1)\left(\frac{\phi}{2}-\frac{h}{2 \mathrm{i}}\right)\right)\right)\right\} Z_{\mathrm{B}}^{2} \\
= & \frac{1}{2 \pi} \int_{0}^{2 \pi} \mathrm{d} \phi \sum_{n=0}^{\infty} \sum_{m=0}^{\infty} \mathrm{e}^{-\boldsymbol{\beta} \delta(n(n+1)+m(m+1)) / 2} \\
& \times\left\{\mathrm{e}^{\mathrm{i}(n+m+1) \phi} \mathrm{e}^{(n-m) h}+\mathrm{e}^{\mathrm{i}(n-m) \phi} \mathrm{e}^{(m+n+1) h}+\mathrm{e}^{\mathrm{i}(m-n) \phi} \mathrm{e}^{(-m-n-1) h}\right. \\
& \left.+\mathrm{e}^{\mathrm{i}(-n-m-1) \phi} \mathrm{e}^{(m-n) h}\right\} Z_{\mathrm{B}}^{2} .
\end{aligned}
$$

Obviously, eq. (AII.5) has non-zero terms only for $n=m$. Therefore, for the equal spacing model, the partition function $Z_{N}^{\text {odd }}(T, H)$ is given by

$$
\exp \left(-\beta E_{N}(0)\right) Z_{N}^{\text {odd }}(T, H)=2 \sum_{n=0}^{\infty} \mathrm{e}^{-\beta \delta n(n+1)} \cosh ((2 n+1) h) Z_{\mathrm{B}}^{2}
$$

in the same way one finds for the "even" case: 


$$
\exp \left(-\beta E_{N}(0)\right) Z_{N}^{\text {even }}(T, H)=\left\{1+2 \sum_{n=0}^{\infty} \mathrm{e}^{-\beta \delta\left\{(n+1)^{2}\right\}} \cosh (2(n+1) h)\right\} Z_{\mathrm{B}}^{2}
$$

The susceptibility $\chi$ is easily calculated from the partition function given in eqs. (AII.6) and (AII.7):

$$
M=-\frac{1}{\mu_{0}} \frac{\partial F}{\partial H}=\frac{1}{\beta \mu_{0}} \frac{\partial}{\partial H} \ln Z_{N}(T, H)
$$

For an odd number of electrons we obtain:

$$
\begin{aligned}
M & =\frac{1}{\beta \mu_{0}} \beta \mu_{0} \mu_{\mathrm{B}} \frac{\partial}{\partial h}\left\{E_{N}(0)+\ln \left(Z_{\mathrm{B}}^{2}\right)+\ln \left(\sum_{n=0}^{\infty} \mathrm{e}^{-\beta \delta n(n+1)}\left(\mathrm{e}^{(2 n+1) h}+\mathrm{e}^{-(2 n+1) h}\right)\right)\right\} \\
& =\mu_{\mathrm{B}} \frac{\sum_{n=0}^{\infty} \mathrm{e}^{-\beta \delta n(n+1)}(2 n+1)\left(\mathrm{e}^{(2 n+1) h}-\mathrm{e}^{-(2 n+1) h}\right)}{\sum_{n=0}^{\infty} \mathrm{e}^{-\beta \delta n(n+1)}\left(\mathrm{e}^{(2 n+1) h}+\mathrm{e}^{-(2 n+1) h}\right)} .
\end{aligned}
$$

If $h \rightarrow 0$, this reduces to:

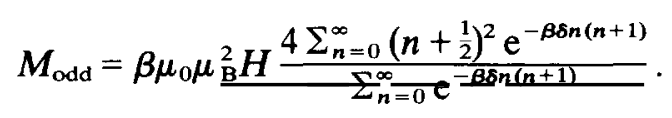

For an even number of electrons, one gets in a similar way:

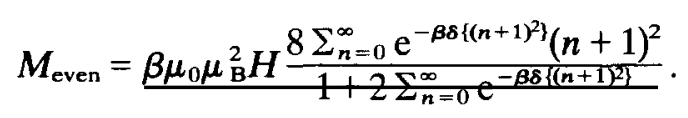

The expressions (AII.10) and (AII.11) can be evaluated for the limiting cases $\beta \delta \ll 1$ and $\beta \delta \gg 1$. In the high temperature limit $(\beta \delta \ll 1)$, the summations can be replaced by integrals, which are of the form $\int_{0}^{\infty} x^{2} \exp \left(-x^{2}\right) \mathrm{d} x=\sqrt{\pi} / 4$, or $\int_{0}^{\infty} \exp \left(-x^{2}\right) \mathrm{d} x=\sqrt{\pi} / 2$. In this limit, one gets for the case with an odd number of electrons:

$$
\chi_{\text {odd }}=\beta \mu_{0} \mu_{\mathrm{B}}^{2} \frac{\left(4 \mathrm{e}^{\beta \delta / 4} / 4 \beta \delta\right) \sqrt{\pi / \beta \delta}}{\frac{1}{2} \mathrm{e}^{\beta \delta / 4} \sqrt{\pi / \beta \delta}}=\frac{2 \mu_{0} \mu_{\mathrm{B}}}{\delta}=\mu_{0} \mu_{\mathrm{B}}^{2} \rho\left(\varepsilon_{\mathrm{F}}\right)
$$

and similarly, for the case with an even number of electrons:

$$
\chi_{\text {even }}=\beta \mu_{0} \mu_{\mathrm{B}}^{2} \frac{(8 / 4 \beta \delta) \sqrt{\pi / \beta \delta}}{1 \mp \sqrt{\pi / \beta \delta}}=\frac{2 \mu_{0} \mu_{\mathrm{B}}^{2}}{\delta}=\mu_{0} \mu_{\mathrm{B}}^{2} \rho\left(\varepsilon_{\mathrm{F}}\right)
$$

where, again, the usual bulk result for the Pauli spin paramagnetism is obtained, for both cases.

In the QSE limit $(\beta \delta \gg 1)$ only very few levels can be reached, and hence only the lowest terms in the summation need to be considered for eqs. (AII.6) and (AII.7). For this case, eqs. (AII.10) and (AII.11) reduce to: 


$$
\begin{aligned}
\chi_{\text {odd }} & =\beta \mu_{0} \mu_{\mathrm{B}}^{2} \frac{1+9 \mathrm{e}^{-2 \beta \delta}+\mathrm{O}\left(\mathrm{e}^{-6 \beta \delta}\right)}{1+\mathrm{e}^{-2 \beta \delta}+\mathrm{O}\left(\mathrm{e}^{-6 \beta \delta}\right)}=\beta \mu_{0} \mu_{\mathrm{B}}^{2}\left\{1+8 \mathrm{e}^{-2 \beta \delta}+\mathrm{O}\left(\mathrm{e}^{-4 \beta \delta}\right)\right\} \\
& =\frac{\mu_{0} \mu_{\mathrm{B}}^{2}}{k T}+\chi_{\text {bulk }}\left(\frac{4 \delta}{k T} \exp \left(-\frac{2 \delta}{k T}\right)\right)
\end{aligned}
$$

and:

$$
\begin{aligned}
\chi_{\text {even }} & =\beta \mu_{0} \mu_{\mathrm{B}}^{2} \frac{8\left(\mathrm{e}^{-\beta \delta}+\mathrm{O}\left(\mathrm{e}^{-4 \beta \delta}\right)\right)}{1+2 \mathrm{e}^{-\beta \delta}+\mathrm{O}\left(\mathrm{e}^{-4 \beta \delta}\right)}=\beta \mu_{0} \mu_{\mathrm{B}}^{2}\left\{8 \mathrm{e}^{-\beta \delta}+\mathrm{O}\left(\mathrm{e}^{-2 \beta \delta}\right)\right\} \\
& =\chi_{\text {bulk }}\left(\frac{4 \delta}{k T} \exp \left(-\frac{\delta}{k T}\right)\right) .
\end{aligned}
$$

The bulk Pauli spin paramagnetism is attenuated exponentially, and for odd number of electrons the low temperature susceptibility is dominated by the susceptibility of the one free spin per particle.

\section{References}

[1] G. Mie, Ann. Phys. (Leipz.) 25 (1908) 377.

[2] H. Fröhlich, Physica (Utr.) 4 (1937) 406.

[3] R. Kubo, J. Phys. Soc. Jap. 17 (1962) 975.

[4] L.P. Gor'kov and G.M. Eliashberg, Zh. Eksp. Teor. Fiz. 48 (1965) 1407 [English translation: Sov. Phys.-JETP 21 (1965) 940].

[5] Int. Meeting on the Small Particles and Inorganic Clusters, J. Phys. (Paris) 38, Colloque C-2 (1977).

[6] Second Int. Meeting on the Small Particles and Inorganic Clusters, Surf. Sci. 106 (1981).

[7] H.P. Baltes and E. Simanek, Physics of Microparticles, to appear in: Aerosol Microphysics II, ed. W. Marlow, Topics in Current Physics (Springer Verlag, Berlin)

[8] A.E. Hughes and S.C. Jain, Adv. Phys. 28 (1979) 717.

[9] L. Genzel, Festkörperprobleme 14 (1974) 183.

[10] W.D. Knight, J. Vac. Sci. Technol. 10 (1973) 705.

[11] L.D. Landau and E.M. Lifshitz, Statistical Physics (3rd ed., Pergamon Press, London, 1959) p. 28.

[12] A. Kawabata, J. Phys. Soc. Jap. 29 (1970) 902.

[13] J.C.M. Garnett, Philos. Trans. R. Soc. Lond. 203 (1904) 385; 205 (1906) 237.

[14] A.P. van Gelder, J. Holvast. J.H.M. Stoelinga and P. Wyder, J. Phys. C 5 (1972) 2757.

[15] P. Debye, Ann. Phys. (Leipz.) 30 (1909) 57.

[16] M. Born, Optik (J. Springer, Berlin, 1933).

[17] H.C. van de Hulst, Light scattering by small particles (J. Wiley \& Sons, New York, 1957)

[18] C. v. Fragstein and H. Römer, Z. Phys. 151 (1958) 54.

[19] W.T. Doyle, Phys. Rev. 111 (1958) 1067.

[20] W. Hampe, Z. Phys. 152 (1958) 476.

[21] R.H. Doremus, J. Chem. Phys. 40 (1964) 2389.

[22] R.H. Doremus, J. Chem. Phys. 42 (1965) 414.

[23] R.H. Doremus, J. Appl. Phys. 37 (1966) 2775

[24] U. Kreibig and C. v. Fragstein, Z. Phys. 224 (1969) 307

[25] U. Kreibig, J. Phys. F 4 (1974) 999.

[26] L. Genzel, T.P. Martin and U. Kreibig, Z. Phys. B 21 (1975) 339.

[27] U. Kreibig, J. Phys. (Paris) 38 (1977) C2-97.

[28] M.A. Smithard and R. Dupree, Phys. Status Solidi A 11 (1972) 695.

[29] M.A. Smithard, Solid State Commun. 13 (1973) 153.

[30] M.A. Smithard and M.Q. Tran, Helv. Phys. Acta 46 (1974) 869.

[31] M.A. Smithard, Solid State Commun. 14 (1974) 407

[32] S.C. Jain and N.D. Arora, J. Phys. Chem. Solids 35 (1974) 1231. 
[33] C.G. Granqvist and O. Hunderi, Phys. Rev. B 16 (1977) 3513.

[34] S. Norrman, T. Andersson, C.G. Granquist and O. Hunderi, Phys. Rev. B 18 (1978) 674.

[35] C.G. Granqvist, N. Calander and O. Hunderi, Solid State Commun. 31 (1979) 249.

[36] U. Kreibig and P. Zacharias, Z. Phys. 231 (1970) 128.

[37] F. Fujimoto and K. Komaki, J. Phys. Soc. Jap. 25 (1968) 1679.

[38] D.M. Mann and H.P. Broida, J. Appl. Phys. 44 (1973) 4950;

J.D. Eversole and H.P. Broida, Phys. Rev. B 15 (1977) 1644.

[39] R.W. Rendell, D.J. Scalapino and B. Mühlschlegel, Phys. Rev. Lett. 41 (1978) 1746.

[40] A. Kawabata and R. Kubo, J. Phys. Soc. Jap. 21 (1966) 1765.

[41] L. Sander, J. Phys. Chem. Solids 29 (1968) 291.

[42] M. Cini and P. Ascarelli, J. Phys. F 4 (1974) 1998.

[43] A.A. Lushnikov and A.J. Simonov, Z. Phys. 270 (1974) 17.

[44] R. Ruppin and H. Yatom, Phys. Status Solidi B 74 (1976) 647.

[45] U. Kreibig, Z. Phys. B 31 (1978) 39.

[46] L. Genzel and U. Kreibig, Z. Phys. B 37 (1980) 93.

[47] J.-D. Ganière, R. Rechsteiner and M.A. Smithard, Solid State Commun. 16 (1975) 113.

[48] P. Ascarelli and M. Cini, Solid State Commun. 19 (1976) 385.

[49] R. Ruppin, J. Phys. Chem. Solids 39 (1978) 233.

[50] A.A. Lushnikov, V.V. Maksimenko and A.J. Simcnov, Solid State Commun. 20 (1976) 545.

[51] C.G. Granqvist and O. Hunderi, Z. Phys. B 30 (1978) 47.

[52] R. Clanget, Optik 35 (1972) 180;

R. Ruppin, Phys. Rev. B 11 (1975) 2871.

[53] R. Ruppin, Phys. Status Solidi B 87 (1978) 619.

[54] R. Landauer, in: Electrical Transport and Optical Properties of Inhomogeneous Media, eds. J.C. Garland and D.B. Tanner, AIP Conf. Proc. 40 (American Institute of Physics, New York, 1978) p. 2.

[55] D.K. Hale, J. Mater. Sci. 11 (1976) 2105.

[56] Z. Hashin and S. Shtrikman, J. Appl. Phys. 33 (1962) 3125.

[57] D.A.G. Bruggeman, Ann. Phys. (Leipz.) 24 (1935) 636.

[58] J.I. Gittleman and B. Abeles, Phys. Rev. B 15 (1977) 3273.

[59] I. Webman, J. Jortner and M.H. Cohen, Phys. Rev. B 15 (1977) 5712.

[60] C.G. Granquist and O. Hunderi, Phys. Rev. B 18 (1978) 2897.

[61] D. Polder and J.H. van Santen, Physica (Utr.) 12 (1946) 257.

[62] F.L. Galeener, Phys. Rev. Lett. 27 (1971) 421; 27 (1971) 769.

[63] R.W. Cohen, G.D. Cody, M.D. Coutts and B. Abeles, Phys. Rev. B 8 (1973) 3689.

[64] P. O'Neill and A. Ignatiev, Phys. Rev. B 18 (1978) 6540.

[65] C.G. Granquist and G.A. Niklasson, J. Appl. Phys. 49 (1978) 3512.

[66] C.G. Granqvist and O. Hunderi, J. Appl. Phys. 50 (1979) 1058.

[67] P. Clippe, R. Evrard and A.A. Lucas, Phys. Rev. B 14 (1976) 1715.

[68] D. Bedaux and J. Vlieger, Physica (Utr.) 73 (1974) 287.

[69] C.G. Granquist and O. Hunderi, Phys. Rev. B 16 (1977) 1353.

[70] W. Lamb, D.M. Wood and N.W. Ashcroft, Phys. Rev. B 21 (1980) 2248.

[71] C.G. Granquist and O. Hunderi, Phys. Rev. B 18 (1978) 1554.

[72] Ping Sheng, Phys. Rev. Lett. 45 (1980) 60.

[73] R. Baetzold, Comments Solid State Phys. 4 (1971/1972) 62.

[74] R.C. Baetzold and R.E. Mack, J. Chem. Phys. 62 (1975) 1513.

[75] D.R. Salahub and R.P. Messmer, Phys. Rev. B 16 (1977) 2526.

[76] C.F. Melius, T.H. Upton and W.A. Goddard III, Solid State Commun. 28 (1978) 501.

[77] P. Kadura and L. Künne, Phys. Status Solidi B 88 (1978) 537.

[78] M.R. Hoare and P. Pal, Adv. Phys. 24 (1975) 645.

[79] C.L. Briant and J.J. Burton, J. Chem. Phys. 63 (1975) 2045.

[80] C. Kittel, Introduction to Solid State Physics (5th ed., John Wiley \& Sons, New York, 1976) p. 165.

[81] H. Weyl, Rendiconti del Circulo Matematico di Palermo 39 (1915) 1.

[82] H. Weyl, in: Gesammelte Abhandelungen, ed. K. Chaudrasekbaran (Springer Verlag, Berlin, 1968) Vol. I, papers 13; $16-19 ; 22$.

[83] H.P. Baltes and E.R. Hilf, Spectra of Finite Systems (Bibliographisches Institut, Mannheim, 1976).

[84] H.P. Baltes, Infrared Phys. 16 (1976) 1.

[85] F. Schogli, Handbuch der Physik (Springer Verlag, Berlin, 1956) Vol. I, p. 344 ff.

[86] See: ref. [80] p. 162.

[87] L.D. Landau and E.M. Lifshitz, Statistical Physics (3rd ed., Pergamon Press, London, 1959) p. 359.

[88] R. Denton, B. Mühlschlegel and D.J. Scalapino, Phys. Rev. Lett. 26 (1971) 707. 
[89] C.E. Porter and N. Rosenzweig, Suomalaisen Tiedeakatemian, Series A VI, No. 44 (1960).

[90] C.E. Porter, Statistical Theories of Spectra: Fluctuations (Academic Press, New York, 1965). This book contains many reprints of relevant references concerning the theory of random matrices and level statistics.

[91] E.P. Wigner, Ann. Math. 53 (1951) 36; 62 (1955) 548; 65 (1957) 203; 67 (1958) 325.

[92] F.J. Dyson, J. Math. Phys. 3 (1962) 140; 3 (1962) 157; 3 (1962) 166; F.J. Dyson and M.L. Mehta, J. Math. Phys. 4 (1963) 701: M.L. Mehta and F.J. Dyson, J. Math. Phys. 4 (1963) 713.

[93] R. Kubo, in: Polarisation, Matière et Rayonnement, Livre Jubilée en l'honneur du Professor A. Kastler (Presses Universitaires de France, 1969) p. 325.

[94] R. Kubo, Comments Solid State Phys. 1 (1968/1969) 61.

[95] R. Kubo, J. Phys. (Paris) 38 (1977) C2-69.

[96] J. Wishart, Biometrika 20 (1928) 32.

[97] See: ref. [90] p. $53 \mathrm{ff}$.

[98] M. Gaudin, Nucl. Phys. 25 (1961) 447.

[99] C.E. Porter, J. Math. Phys. 4 (1963) 1039.

[100] N. Rosenzweig and C.E. Porter, Phys. Rev. 120 (1960) 1698.

[101] R.J. Elliott, Phys. Rev. 96 (1954) 266.

[102] J. Czerwonko, Phys. Status Solidi 30 (1968) 723.

[103] J. Czerwonko, Phys. Status Solidi 31 (1969) K161.

[104] K. Weron, Acta Phys. Pol. A 53 (1978) 19.

[105] R. Denton, B. Mühlschlegel and D. Scalapino, Phys. Rev. B 7 (1973) 3589.

[106] M.J. Rice, W.R. Schneider and S. Strässler, Phys. Rev. B 8 (1973) 474.

[107] S. Strässler, M.J. Rice and P. Wyder, Phys. Rev. B 6 (1972) 2575.

[108] J.M. Ziman, Principles of the Theory of Solids (Cambridge University Press, 1965) Chap. 8, p. 224.

[109] E.A. Shapoval, Zh. Eksp. Teor. Fiz. 47 (1964) 1007 [English translation: Sov. Phys.-JETP 20 (1965) 675].

[110] R.P. Devaty and A.J. Sievers, Phys. Rev. B 22 (1980) 2123.

[111] See any book on NMR, e.g. C.P. Slichter, Principles of Magnetic Resonance (Harper \& Row, 1965) p. $89 \mathrm{ff}$.

[112] J. Charvolin, C. Froidevaux, C. Taupin and J.M. Winter, Solid State Commun. 4 (1966) 357.

[113] M. Lambert, Ch. Mazières and A. Guinier, J. Phys. Chem. Solids 18 (1961) 129.

[114] C. Taupin, C.R. Acad. Sci. B 262 (1966) 1617.

[115] C. Taupin, J. Phys. Chem. Solids 28 (1967) 41

[116] S. Kobayashi, T. Takahashi and W. Sasaki, Phys. Lett. A 33 (1970) 429.

[117] S. Kobayashi, T. Takahashi and W. Sasaki, J. Phys. Soc. Jap. 31 (1971) 1442.

[118] S. Kobayashi, T. Takahashi and W. Sasaki, J. Phys. Soc. Jap. 36 (1974) 714; 38 (1975) 599.

[119] K. Nomura, S. Kobayashi and W. Sasaki, J. Phys. Soc. Jap. 48 (1980) 37.

[120] K. Nomura, S. Kobayashi and W. Sasaki, Solid State Commun. 24 (1977) 81.

[121] A.A. Abrikosov and L.P. Gor'kov, Zh. Eksp. Teor. Fiz. 42 (1962) 1088 [English translation: Sov. Phys.-JETP 15 (1962) 752].

[122] W.A. Hines and W.D. Knight, Phys. Rev. B 4 (1971) 893.

[123] R.H. Hammond and G.M. Kelly, Phys. Rev. Lett. 18 (1967) 156.

[124] B. Mühlschlegel, D.J. Scalapino and R. Denton, Phys. Rev. B 6 (1972) 1767.

[125] M. Ido and R. Hoshino. J. Phys. Soc. Jap. 38 (1975) 898.

[126] M. Ido, J. Phys. Soc. Jap. 41 (1976) 412.

[127] H. Shiba. J. Low Temp. Phys. 22 (1976) 105.

[128] T. Takahashi, S. Kobayashi and W. Sasaki, Solid State Commun. 17 (1975) 681.

[129] J. Sone, J. Low Temp. Phys. 23 (1976) 699.

[130] J. Sone, J. Phys. Soc. Jap. 42 (1977) 1457.

[131] P.K. Tse and D.E. MacLaughlin. Solid State Commun. 26 (1978) 151.

[132] P.K. Tse and D.E. MacLaughlin, J. Low Temp. Phys. 39 (1980) 339.

[133] S. Kobayashi, T. Takahashi and W. Sasaki, J. Phys. Soc. Jap. 32 (1972) 1234.

[134] P. Yee and W.D. Knight, Phys. Rev. B 11 (1975) 3261.

[135] M. Ido and R. Hoshino, J. Phys. Soc. Jap. 36 (1974) 1325.

[136] W.A. Hines, in: Proc. 12th Int. Conf. Low Temp. Phys., LT12, Kyoto, 1970, ed. E. Kanda (Academic Press of Japan, 1971 ) p. 591.

[137] R.J. Charles and W.A. Harrison, Phys. Rev. Lett. 11 (1963) 75.

[138] I. Yu, A.A.V. Gibson, E.R. Hunt and W.P. Halperin, Phys. Rev. Lett. 44 (1980) 348.

[139] B.W. Holland, in: Proc. 14th Coll. Ampère (North-Holland Publ. Co., Amsterdam, 1967) p. 468.

[140] S. Schultz and C. Latham. Phys. Rev. Lett. 15 (1965) 148.

[141] M.Ya. Gen and V.I. Petinov, Zh. Eksp. Teor. Fiz. 48 (1965) 29 [English translation: Sov. Phys.-JETP 21 (1965) 19].

[142] C. Taupin and D. Taupin, C.R. Acad. Sci. B 264 (1967) 581.

[143] K. Asayama and Y. Oda, J. Phys. Soc. Jap. 22 (1967) 937. 
[144] A.J. Watts and J.E. Cousins, Phys. Status Solidi 30 (1968) 105.

[145] K. Saiki, T. Fujita, Y. Shimizu, S. Sakoh and N. Wada, J. Phys. Soc. Jap. 32 (1972) 447.

[146] J.-P. Borel, C. Borel-Narbel and R. Monot, Helv. Phys. Acta 47 (1974) 537.

[147] V.I. Petinov and A.Yu. Ardashev, Fiz. Tverd. Tela 11 (1969) 3 [English translation: Sov. Phys.-Solid State 11 (1969) 1].

[148] M.A. Smithard, Solid State Commun. 14 (1974) 411.

[149] D.A. Gordon, Phys. Rev. B 13 (1976) 3738.

[150] J.-L. Millet and R. Monot, in: Proc. 18th Ampère Congress, Nottingham (North-Holland Publ. Co., 1974) p. 319

[151] R. Dupree, C.T. Forwood and M.J.A. Smith, Phys. Status Solidi 24 (1967) 525.

[152] R. Monot, A. Châtelain and J.-P. Borel, Phys. Lett. A 34 (1971) 57.

[153] S.C. Jain, N.D. Arora and T.Rs. Reddy, Phys. Lett. A 54 (1975) 53.

[154] R. Monot, C. Narbel and J.-P. Borel, Nuovo Cimento B 19 (1974) 253.

[155] A. Châtelain, J.-L. Millet and R. Monot, J. Appl. Phys. 47 (1976) 3670.

[156] R. Monot and J.-L. Millet, J. Phys. (Paris) 37 (1976) L-45.

[157] D.A. Gordon, R.F. Marzke and W.S. Glaunsinger, J. Phys. (Paris) 38 (1977) C2-87.

[158] D.B. Tanner, A.J. Sievers and R.A. Buhrman, Phys. Rev. B 11 (1975) 1330.

[159] C.G. Granqvist, R.A. Buhrman. J. Wyns and A.J. Sievers, Phys. Rev. Lett. 37 (1976) 625.

[160] C.G. Granquist, R.A. Buhrman, J. Wyns and A.J. Sievers, J. Phys. (Paris) 38 (1977) C2-93.

[161] J.A.A.J. Perenboom and C.M.J. Wijers, unpublished.

[162] R. Zsigmondy, Z. Phys. Chem. (Leipz.) 56 (1906) 65.

[163] A.A. Lushnikov, V.V. Maksimenko and A.Ya. Simonov, Fiz. Tverd. Tela 20 (1978) 505 [English translation: Sov. Phys.-Solid State 20 (1978) 292];

V.V. Maksimenko, A.J. Simonov and A.A. Lushnikov, Phys. Status Solidi B 83 (1977) 377.

[164] D. Stroud and F.P. Pan, Phys. Rev. B 17 (1978) 1602.

[165] E. Simanek, Phys. Rev. Lett. 38 (1977) 1161.

[166] R. Ruppin, Phys. Rev. B 19 (1979) 1318.

[167] A.J. Glick and E.D. Yorke, Phys. Rev. 18 (1978) 2490.

[168] C.G. Granqvist, Z. Phys. B 30 (1978) 29.

[169] F. Meier and P. Wyder, Phys. Lett. A 39 (1972) 51.

[170] R. Dupree and M.A. Smithard, J. Phys. C $5(1972) 408$.

[171] H. Akoh and A. Tasaki, J. Phys. Soc. Jap. 42 (1977) 791.

[172] R.F. Marzke, W.S. Glaunsinger and M. Bayard, Solid State Commun. 18 (1976) 1025.

[173] F. Meier and P. Wyder, in: Proc. 13th Int. Conf. Low Temp. Phys., LT13, Boulder, 1972, eds. K.D. Timmerhaus, W.J. O'Sullivan and E.F. Hammel (Plenum Press, New York, 1972) Vol. 2, p. 613.

[174] F. Meier and P. Wyder, Phys. Rev. Lett. 30 (1973) 181.

[175] A.P. van Gelder, Phys. Rev. B 10 (1974) 2144.

[176] J.A.A.J. Perenboom, Magnetic properties of small metallic particles and of some organo-metallic clusters (thesis, Nijmegen, 1979).

[177] J.A.A.J. Perenboom, P. Wyder and F. Meier, Phys. Rev. B 23 (1981) 279.

[178] D. Shoenberg, Superconductivity (Cambridge University Press, 1962).

[179] V.L. Ginzburg. Phys. Lett. 13 (1964) 101.

[180] M. Tinkham, Introduction to Superconductivity (McGraw-Hill, 1975).

[181] J. Bardeen, L.N. Cooper and J.R. Schrieffer, Phys. Rev. 108 (1957) 1175.

[182] A.A. Abrikosov, L.P. Gor'kov and I.E. Dzyaloshinski, Methods of Quantum Field Theory in Statistical Physics (Prentice-Hall, Englewood Cliffs, N.J., 1963) Chap. 7.

[183] P.G. De Gennes, Rev. Mod. Phys. 36 (1964) 312.

[184] S. Matsuo, H. Sugiura and S. Noguchi, J. Low Temp. Phys. 15 (1974) 481.

[185] V.L. Ginzburg, Zh. Eksp. Teor. Fiz. 34 (1958) 113 [English translation: Sov. Phys.-JETP 7 (1958) 78].

[186] F. London, Superfluids (Dover Publications, New York, 1961) Vol. 1.

[187] A.M. Clogston, Phys. Rev. Lett. 9 (1962) 266.

[188] A. Rothwarf, Phys. Lett. A 30 (1969) 55.

[189] J.M. Dickey and A. Paskin, Phys. Rev. B 1 (1970) 851.

[190] M. Strongin, O.F. Kammerer, J.E. Crow, R.D. Parks, D.H. Douglass Jr. and M.A. Jensen, Phys. Rev. Lett. 21 (1968) 1320.

[191] O.F. Kammerer and M. Strongin, Phys. Lett. 17 (1965) 224.

[192] M. Strongin, A. Paskin, O.F. Kammerer and M. Garber, Phys. Rev. Lett. 14 (1965) 362.

[193] A.I. Larkin, Zh. Eksp. Teor. Fiz. 48 (1965) 232 [English translation: Sov. Phys.-JETP 21 (1965) 153].

[194] K. Maki, Prog. Theor. Phys. 29 (1963) 10; 29 (1963) 333; 29 (1963) 603.

[195] S. Strässler and P. Wyder, Phys. Rev. 158 (1967) 319.

[196] A.A. Abrikosov and L.P. Gorkov, Zh. Eksp. Teor. Fiz. 39 (1960) 1781 [English translation: Sov. Phys.-JETP 12 (1961) 1243].

[197] J. Millstein and M. Tinkham, Phys. Rev. 158 (1967) 325.

[198] P.G. De Gennes and M. Tinkham, Physics (N.Y.) 1 (1964) 107 
[199] P.G. De Gennes, Rev. Mod. Phys. 36 (1964) 225

[200] Y. Nambu and S.F. Tuan, Phys. Rev. 133 (1964) A1.

[201] Y. Nambu and S.F. Tuan, Rev. Mod. Phys. 36 (1964) 288.

[202] D. Shoenberg, Proc. R. Soc. A 175 (1940) 49.

[203] C.S. Whitehead, Proc. R. Soc. A 238 (1956) 175.

[204] Yu.G. Morozov, I.G. Naumenko and V.I. Petinov, Fiz. Tverd. Tela 17 (1975) 2273 [English translation: Sov. Phys.-Solid State 17 (1976) 1504].

[205] R.A. Buhrman and C.G. Granquist, J. Appl. Phys. 47 (1976) 2220.

[206] Yu.N. Ovchinnikov, Zh. Eksp. Teor. Fiz. 57 (1969) 894 [English translation: Sov. Phys.-JETP 30 (1970) 489].

[207] I. Giaever and H.R. Zeller, Phys. Rev. Lett. 20 (1968) 1504; H.R. Zeller and I. Giaever, Phys. Rev. 181 (1969) 789.

[208] H.R. Zeller and I. Giaever, in: Superconductivity, Proc. Int. Conf. on Science of Superconductivity, Stanford 1969, ed. F. Chilton (North-Holland Publ. Co., Amsterdam, 1971) p. 173.

[209] F. Wright Jr., Phys. Rev. 163 (1967) 420.

[210] J.M. Dickey and A. Paskin, Phys. Rev. Lett. 21 (1968) 1441.

[211] S. Akselrod, M. Pasternak and S. Bukshpan, J. Low Temp. Phys. 17 (1974) 375.

[212] M.H. Cohen and D.H. Douglass Jr., Phys. Rev. Lett. 19 (1967) 118.

[213] P.W. Anderson, J. Phys. Chem. Solids 11 (1959) 26.

[214] J.M. Blatt and C.J. Thompson, Phys. Rev. Lett. 10 (1963) 332

[215] C.J. Thompson and J.M. Blatt, Phys. Lett. 5 (1963) 6.

[216] Yu.F. Komnik, E.I. Bukhshtab and K.K. Man'kovskii, Zh. Eksp. Teor. Fiz. 57 (1969) 1495 [English translation: Sov. Phys.-JETP 30 (1970) 807].

[217] E.A. Shapoval, Zh. Eksp. Teor. Fiz. Pis'ma Red. 5 (1967) 57 [English translation: JETP Lett. 5 (1967) 45].

[218] R.H. Parmenter, Phys. Rev. 166 (1968) 392.

[219] W. Buckel and R. Hilsch. Z. Phys. 138 (1954) 109.

[220] I.S. Khukhareva, Zh. Eksp. Teor. Fiz. 43 (1962) 1173 [English translation: Sov. Phys.-JETP 16 (1963) 828].

[221] B. Abeles, R.W. Cohen and G.W. Cullen, Phys. Rev. Lett. 17 (1966) 632

[222] R.W. Cohen, B. Abeles and G.S. Weisbarth, Phys. Rev. Lett. 18 (1967) 336.

[223] J.H.P. Watson, Phys. Rev. 148 (1966) 223.

[224] R.H. Parmenter, Phys. Rev. 132 (1963) 2490

[225] D. Markowitz, Physics (N.Y.) 3 (1967) 199.

[226] F. Reif, Phys. Rev. 106 (1957) 208.

[227] W.J. Skocpol and M. Tinkham, Rep. Prog. Phys. 38 (1975) 1049.

[228] K. Huang, Statistical Mechanics (J. Wiley \& Sons, New York, 1963).

[229] R. Brout, Phase Transitions (W.A. Benjamin, New York, 1965).

[230] S. Strässler, Eine einfache Einführung in die Random-Phase-Approximation (Brown Boveri Forschungsbericht KLR-71-09, Brown Boveri Forschungszentrum, Baden, Schweiz).

[231] V.V. Shmidt, Zh. Eksp. Teor. Fiz. Pis'ma Red. 3 (1966) 141 [English translation: JETP Lett. 3 (1966) 89].

[232] J.B. Parkinson, J. Phys. F 2 (1972) 966.

[233] R.A. Buhrman and W.P. Halperin, Phys. Rev. Lett. 30 (1973) 692.

[234] J.P. Hurault and K. Maki, Phys. Rev. B 2 (1970) 2560.

[235] H. Takayama, Prog. Theor. Phys. 48 (1972) 758.

[236] L. Gunther, G. Deutscher and Y. Imry, Phys. Rev. B 7 (1973) 3393.

[237] T. Tsuboi and T. Suzuki, J. Phys. Soc. Jap. 42 (1977) 437.

[238] T. Suzuki and T. Tsuboi, J. Phys. Soc. Jap. 42 (1977) 444.

[239] R.L. Filler, P. Lindenfeld, T. Worthington and G. Deutscher, Phys. Rev, B 21 (1980) 5031.

[240] G. Deutscher, O. Entin-Wohlman, S. Fishman and Y. Shapira, Phys. Rev. B 21 (1980) 5041.

[241] V. Novotny, P.P.M. Meincke and J.H.P. Watson, Phys. Rev. Lett. 28 (1972) 901.

[242] V. Novotny and P.P.M. Meincke, Phys. Rev. B 8 (1973) 4186.

[243] G.H. Comsa, D. Heitkamp and H.S. Räde, Solid State Commun. 20 (1976) 877.

[244] J.M. Basset and R. Ugo in: Aspects of Homogeneous Catalysis, ed. R. Ugo (D. Reidel Publishing Company, Dordrecht, 1977 ) Vol. 3, p. 137.

[245] E.L. Muetterties, T.N. Rhodin, Elliot Band, C.F. Brucker and W.R. Pretzer, Chem. Rev. 79 (1979) 91.

[246] S.W. Marshall and R.M. Wilenzick, Phys. Rev. Lett. 16 (1966) 219.

[247] T. Svedberg, Kolloid-Z. 24 (1919) 1.

[248] J. Turkevich, P.C. Stevenson and J. Hillier, Discuss. Faraday Soc. 11 (1951) 55.

[249] R.M. Wilenzick, D.C. Russell, R.H. Morriss and S.W. Marshall, J. Chem. Phys. 47 (1967) 533.

[250] F.A. Meier, Small Particles and Boundary Conditions (thesis, Nijmegen, 1973).

[251] R. Feick, Kolloid-Z. 36 (1925) 257.

[252] See e.g.: J.A. Venables, Thin Solid Films 32 (1976) 135.

[253] H. Schmeisser, Thin Solid Films 22 (1974) 83. 
[254] C.G. Granqvist and R.A. Buhrman, Appl. Phys. Lett. 27 (1975) 693.

[255] L. Holland, Vacuum Deposition of Thin Films (Chapman and Hall, London, 1966).

[256] H.R. Zeller and D. Kuse, J. Appl. Phys. 44 (1973) 2763.

[257] T. Tsuboi, J. Phys. Soc. Jap. 38 (1975) 590.

[258] I.Yu. Bezotosnyi, V.M. Epikhin and I.N. Nikolaev, Fiz. Tverd. Tela 19 (1977) 3113 [English translation: Sov. Phys.-Solid State 19 (1977) 1819].

[259] R.W. Cohen and B. Abeles, Phys. Rev. 168 (1968) 444.

[260] G. Deutscher, M. Gershenson, E. Grünbaum and Y. Imry, J. Vac. Sci. Technol. 10 (1973) 697.

[261] S. Akselrod, M. Pasternak and S. Bukshpan, Phys. Rev. B 11 (1975) 1040.

[262] B. Abeles, Ping Sheng, M.D. Coutts and Y. Arie, Adv. Phys. 24 (1975) 407.

[263] A.H. Pfund, J. Opt. Soc. Am. 23 (1933) 375.

[264] K. Kimoto, Y. Kamiya, M. Nonoyama and R. Uyeda, Jap. J. Appl. Phys. 2 (1963) 702.

[265] K. Kimoto and I. Nishida, Jap. J. Appl. Phys. 6 (1967) 1047.

[266] N. Wada, Jap. J. Appl. Phys. 6 (1967) 553; 7 (1968) 1287.

[267] M.Ya. Gen, M.S. Ziskin and Yu.I. Petrov, Dokl. Akad. Nauk SSSR 127 (1959) 366.

[268] S. Yatsuya, R. Uyeda and Y. Fukano, Jap. J. Appl. Phys. 11 (1972) 408.

[269] S. Yatsuya, S. Kasukabe and R. Uyeda, Jap. J. Appl. Phys. 12 (1973) 1675.

[270] C.G. Granqvist and R.A. Buhrman, J. Appl. Phys. 47 (1976) 2200.

[271] C.G. Granqvist and R.A. Buhrman, Solid State Commun. 18 (1976) 123.

[272] R. Uyeda, J. Cryst. Growth 24/25 (1974) 69.

[273] M.Ya. Gen, E.A. Velichenkova, I.V. Eremina and M.S. Zuskin, Fiz. Tverd. Tela 6 (1964) 1622 [English translation: Sov. Phys,-Solid State 6 (1964) 1274].

[274] A. Tasaki, M. Takao and H. Tokunaga, Jap. J. Appl. Phys. 13 (1974) 271.

[275] T. Fujita, K. Ohshima, N. Wada and T. Sakakibara, J. Phys. Soc. Jap. 29 (1970) 797.

[276] S. Matsuo, H. Miyata and S. Noguchi, Jap. J. Appl. Phys. 13 (1974) 351.

[277] S. Takajo, S. Kobayashi and W. Sasaki, J. Phys. Soc. Jap. 35 (1973) 712.

[278] G.H. Comsa, D. Heitkamp and H.S. Räde, Solid State Commun. 24 (1977) 547.

[279] M. Rappaz and F. Faes, J. Appl. Phys. 46 (1975) 3273.

[280] N. Wada and M. Ichikawa, Jap. J. Appl. Phys. 15 (1976) 755.

[281] T. Fujita, K. Ohshima and T. Kuroishi, J. Phys. Soc. Jap. 40 (1976) 90.

[282] Yu.G. Morozov, I.G. Naumenko and V.I. Petinov, Fiz. Tverd. Tela 16 (1974) 2621; 16 (1974) 3057 [English translation: Sov. Phys.-Solid State 16 (1975) $1701 ; 16$ (1975) 1974].

[283] I.G. Naumenko, V.I. Petinov and M.Ya. Gen, Fiz. Tverd. Tela 13 (1971) 3260 [English translation: Sov. Phys.-Solid State 13 (1972) 2740 ].

[284] I.P. Suzdalev, M.Ya. Gen, V.I. Gol'danskii and E.F. Makarov, Zh. Eksp. Teor. Fiz. 51 (1966) 118 [English translation: Sov. Phys.-JETP 24 (1967) 79].

[285] J.D. Eversole and H.P. Broida, J. Appl. Phys. 45 (1974) 596.

[286] S. Yatsuya, K. Mihama and R. Uyeda, Jap. J. Appl. Phys. 13 (1974) 749.

[287] O.F. Hagena and W. Obert, J. Chem. Phys. 56 (1972) 1793.

[288] G.D. Stein and J.A. Armstrong, J. Chem. Phys. 58 (1973) 1999.

[289] B. Raoult and J. Farges, Rev. Sci. Instrum. 44 (1973) 430.

[290] J. Farges, M.F. de Feraudy, B. Raoult and G. Torchet, J. Phys. (Paris) 38 (1977) C2-47.

[291] R.A. Larson, S.K. Neoh and D.R. Herschbach, Rev. Sci. Instrum. 45 (1974) 1511.

[292] W.D. Knight, R. Monot, E.R. Dietz and A.R. George, Phys. Rev. Lett. 40 (1978) 1324.

[293] A. Yokozeki and G.D. Stein, J. Appl. Phys. 49 (1978) 2224.

[294] K. Sattler, J. Mühlbach and E. Recknagel, Phys. Rev. Lett. 45 (1980) 821.

[295] R.D. Maurer, J. Appl. Phys. 29 (1958) 1.

[296] S.D. Stookey, J. Am. Ceram. Soc. 32 (1949) 246.

[297] U. Kreibig, Appl. Phys. 10 (1976) 255.

[298] W. Schulze, H.U. Becker and D. Leutloff, J. Phys. (Paris) 38 (1977) C2-7.

[299] G.A. Ozin and H. Huber, Inorg. Chem. 17 (1978) 155.

[300] M. Lambert and A. Guinier, J. Phys. (Paris) 24 (1963) 552.

[301] J.M. Calleja and F. Agullo-Lopez, Phys. Status Solidi A 25 (1974) 473.

[302] S.C. Jain and N.D. Arora, J. Phys. Soc. Jap. 36 (1974) 739.

[303] J.M. Calleja and F. Agullo-Lopez, Phys. Lett. A 53 (1975) 317.

[304] A.E. Hughes and S.C. Jain, Phys. Lett. A 58 (1976) 61.

[305] R.H. Lindquist, G. Constabaris, W. Kündig and A.M. Portis, J. Appl. Phys. 39 (1968) 1001.

[306] V. Novotny, T.M. Holden and G. Dolling, Can. J. Phys. 52 (1974) 748.

[307] J.H.P. Watson, Phys. Rev. B 2 (1970) 1282.

[308] V.N. Bogomolov, R. Sh. Malkovich and F.A. Chudnovskii, Fiz. Tverd. Tela 11 (1969) 3053 [English translation: Sov. Phys.-Solid State 11 (1970) 2483]. 
[309] V.N. Bogomolov, R. Sh. Malkovich and F.A. Chudnovskii, Fiz. Tverd. Tela 11 (1969) 2835 [English translation: Sov. Phys.-Solid State 11 (1970) 2295].

[310] V.N. Bogomolov, Fiz. Tverd. Tela 13 (1971) 815; 14 (1972) 1228 [English translation: Sov. Phys.-Solid State 13 (1971) 672; 14 (1972) 1048].

[311] V.N. Bogomolov and A.I. Zadorozhnii, Fiz. Tverd. Tela 13 (1971) 2771 [English translation: Sov. Phys.-Solid State 13 (1972) 2317].

[312] See e.g.: R. Becker, Theorie der Wärme (Springer Verlag, Berlin, 1966) p. 165.

[313] A. Sommerfeld, Thermodynamik und Statistik (2nd ed., Akademische Verlagsgesellschaft, Geest \& Portig, Leipzig, 1962) p. 221.

[314] See: ref. [80] p. 450.

[315] E.T. Whittaker and G.N. Watson, A Course of Modern Analysis (Cambridge University Press, London, 1927). 